\title{
Attenuated apoptosis as consequence of Epithelial Mesenchymal Transition
}

\section{Dissertation}

\author{
for the award of the degree \\ “Doctor rerum naturalium” (Dr. rer. nat.) \\ in the "Biology of Cells" Program \\ at the Georg August University Göttingen, \\ Faculty of Biology
}

submitted by

Ulrike Keitel

born in

Leipzig , Germany

Göttingen, 2013 


\section{PhD Thesis Committee:}

Prof. Dr. Matthias Dobbelstein, Faculty of Medicine, University of Göttingen (reviewer) Prof. Dr. Andreas Wodarz, Faculty of Medicine, University of Göttingen (reviewer) Prof. Dr. Felix Brembeck, Faculty of Medicine, University of Göttingen

Date of oral exam: $\quad$ September 09, 2013 


\section{Affidavit}

Herewith I declare that I prepared the $\mathrm{PhD}$ Thesis „Attenuated apoptosis as a consequence of Epithelial Mesenchymal Transition” on my own and with no other sources and aids than quoted.

Göttingen, 29.07.2013 


\section{Acknowledgements}

First of all, I would like to thank my supervisor Prof. Matthias Dobbelstein for his support and guidance through the last years. He always encouraged my ideas, supported my initiatives and gave me the freedom to realize my own scientific ideas. Moreover, I am very grateful for his appreciation of my work and all the things I have learned about cancer research and science in general.

Further, I would like to thank Prof. Andreas Wodarz and Prof. Felix Brembeck for being in my thesis committee and that they were on hand with help and advice during the progress of this work, but also for their genuine interest in my project. I also would like to thank my extended committee members Prof. Heidi Hahn, Prof. Peter Burfeind and Prof. Uwe-Karsten Hanisch.

I would like to thank those who contributed to this project: Prof. Robert Weinberg (MIT Boston, USA) and Dr. Christina Scheel (Institute for Stem Cell Research, Helmholtz Center Munich), for providing us with the HMLE (RAS) cell systems, Dr. Andreas Scheel (Institute for Pathology Nordhessen) for doing immunohistochemistry of human breast cancer tissues and his precise work in preparing the data, Prof. Jürgen Thomale (Institute for Cellbiology, University of Duisburg-Essen) for performing the Immuno-Slot-Plot assay and Dr. Silke Kaulfuß (Institute for Humangenetics, University of Göttingen) for doing the xenograft experiments.

I greatly appreciate the support of the Göttingen Graduate School for Neurosciences, Biophysics and Molecular Biosciences (GGNB) in providing me with many helpful courses and their friendly helpfulness. Further, I thank the Mentoring Program of the Universitätsmedizin Göttingen, which imparted me many soft skills during excellent workshops.

I would not have been able to accomplish my thesis without my great present and former lab members of the Department of Molecular Oncology, always enabling me to practice science in an enjoyable, friendly and helpful atmosphere. I thank you all very much indeed for your scientific support, great discussions, good ideas and many cheerful moments during the last years. And I am grateful for all the friendships that arose out of these exciting years here in Göttingen.

I especially thank Cathrin and Antje, who did the initial experiments to start this project and for their inexhaustible support for me and my work.

I thank Kamila, Karola, Claudia and Kathrin for their lab and administrative organization, and also Patricia for her administrative know-how and personal advice during the last years.

I am grateful to Prof. Ute Moll for great discussions and her expert knowledge.

I also want to thank my friends in- and outside Göttingen for encouraging, supporting and motivating me, but also for having many funny times.

I would like to express my gratitude to my family: Thank you for your support and openness to my way of doing.

Special thanks I want to address to Moritz for the constant encouragement, sharing highs and lows of my work and the fondly, motivating words you always found for me. Thank you! 


\section{Table of contents}

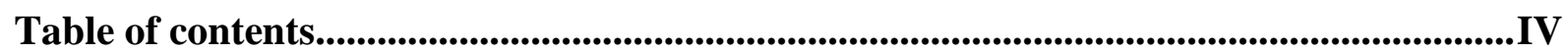

List of figures .....................................................................................................................................VIII

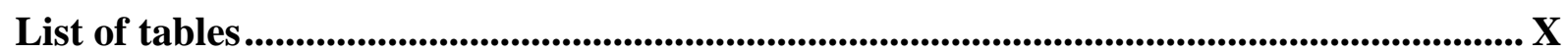

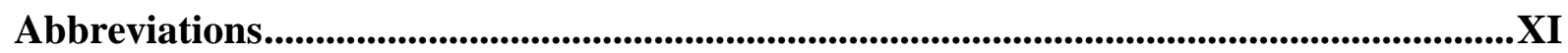

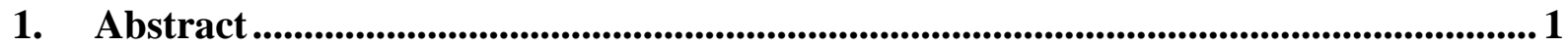

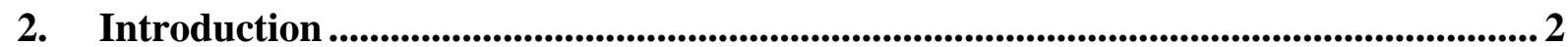

2.1 Need for new cancer therapies to eliminate subpopulations of tumor cells .............. 2

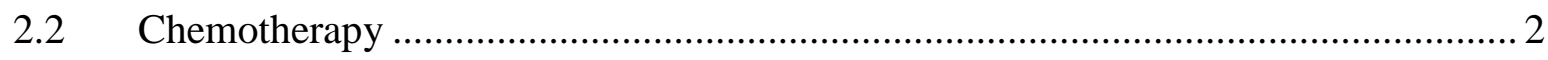

2.2.1 Particularized view on chemotherapy used in this thesis ....................................... 3

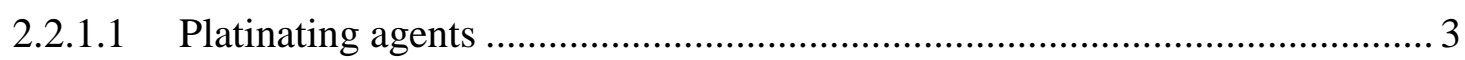

2.2.1.2 Anthracyclines............................................................................

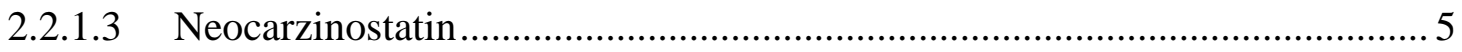

2.2.2 Chemoresistance and mechanisms involved ..................................................... 6

2.2.2.1 Drug influx and efflux .......................................................................

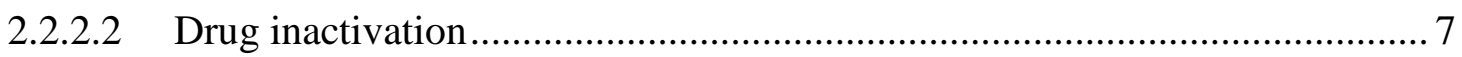

2.2.2.3 Drug targets and regulatory proteins .....................................................

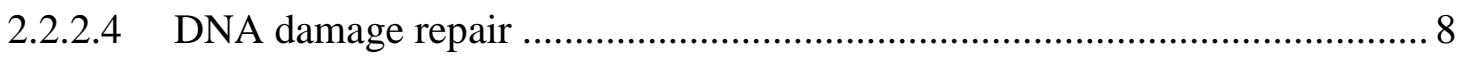

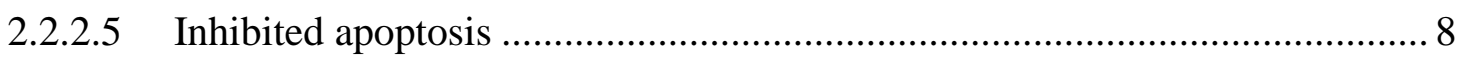

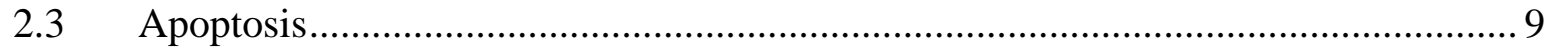

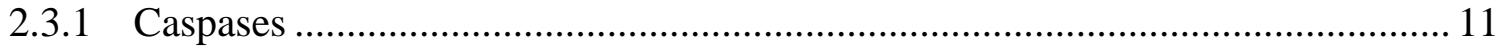

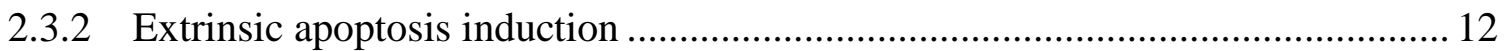

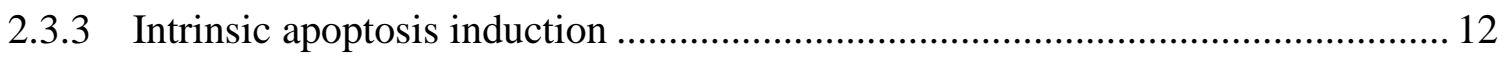

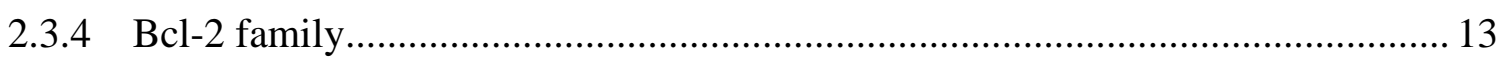

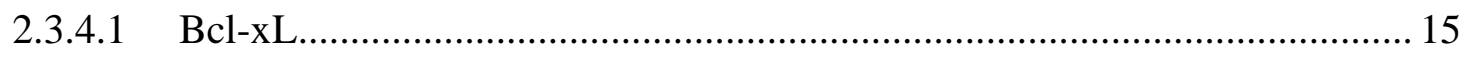

2.4 Epithelial Mesenchymal Transition .................................................................. 16

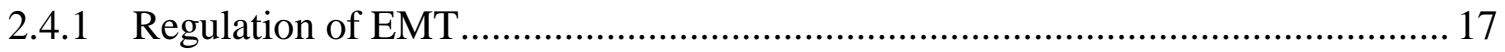

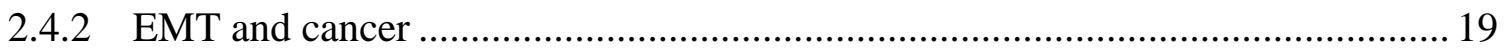

2.4.3 EMT involved in chemoresistance ............................................................. 21

2.4.4 Model system for EMT …………………………………………….... 22

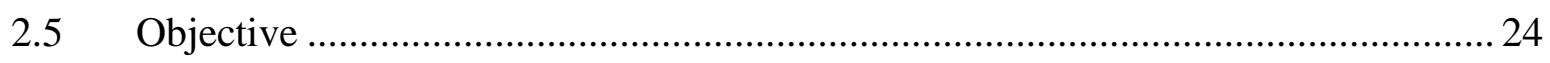

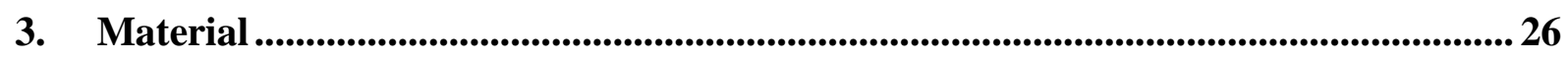

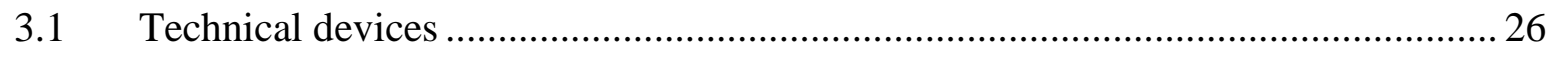




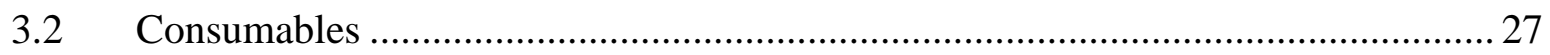

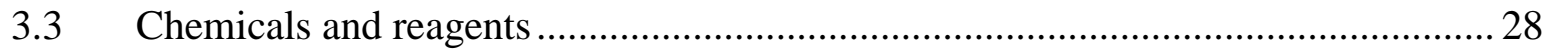

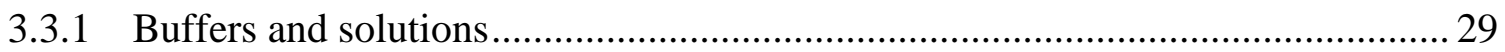

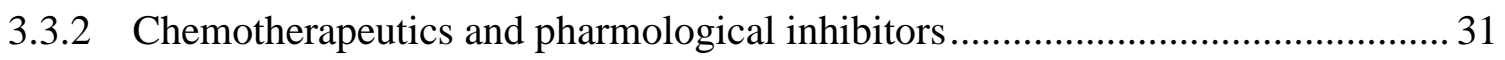

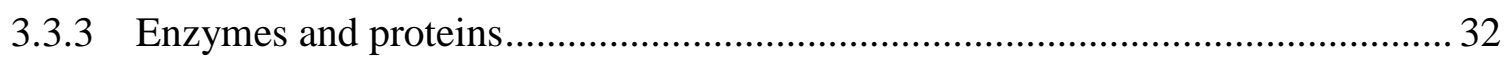

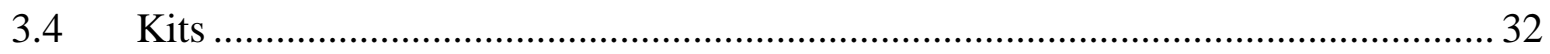

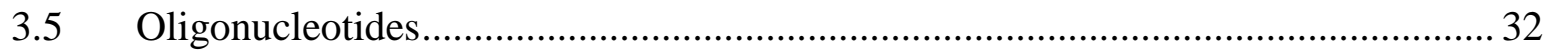

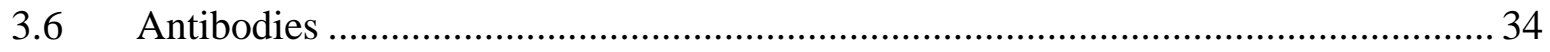

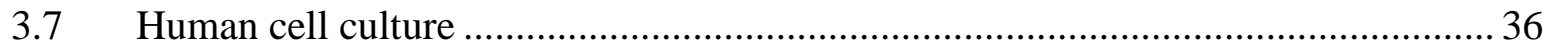

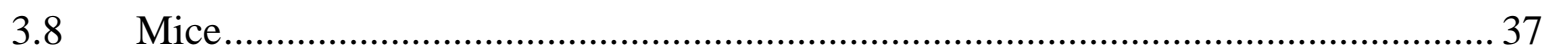

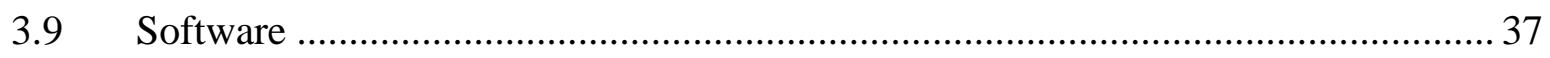

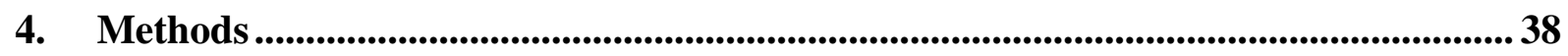

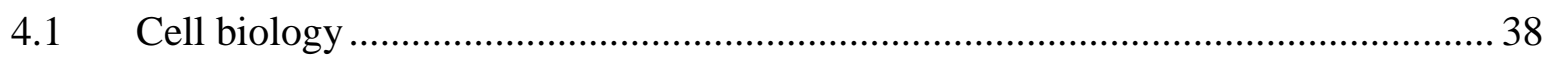

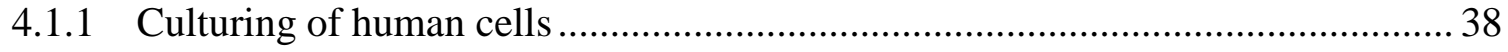

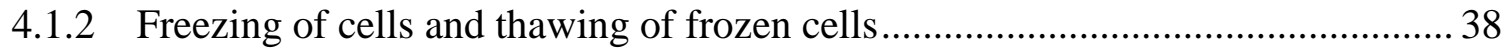

4.1.3 Model system for EMT: HMLE and HMLE RAS cells...................................... 39

4.1.3.1 Generating the HMLE and HMLE RAS system ...................................... 39

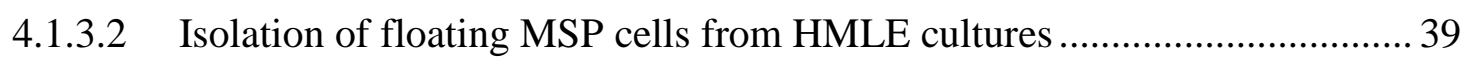

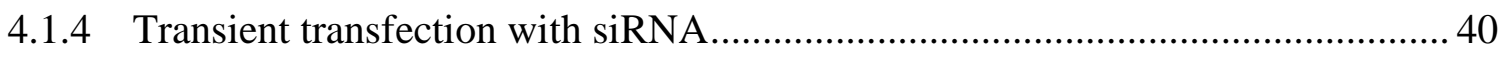

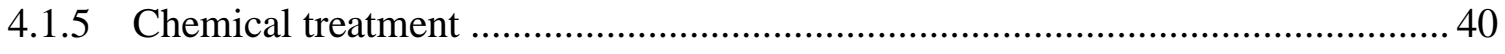

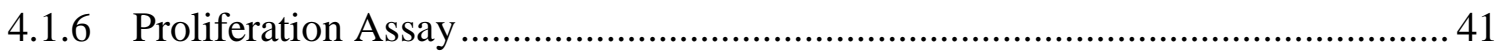

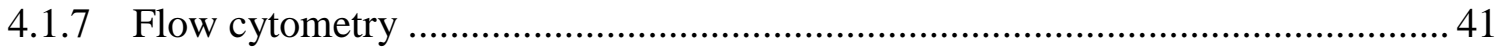

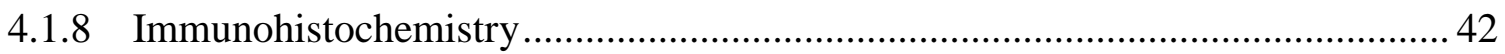

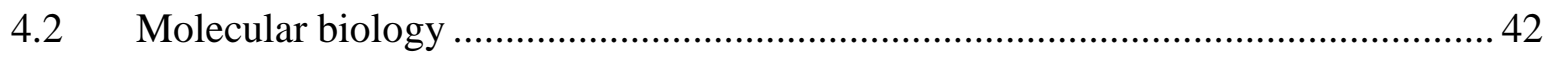

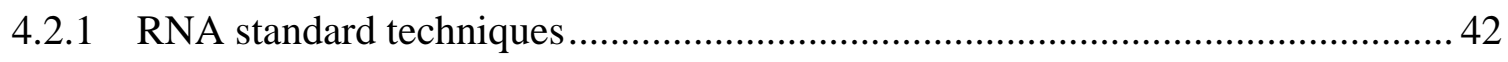

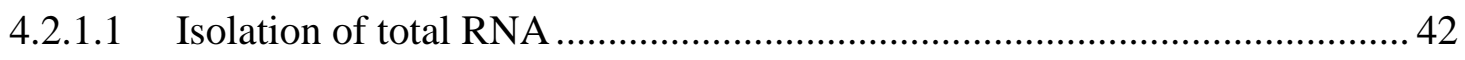

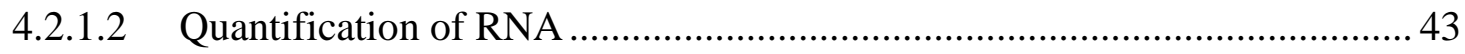

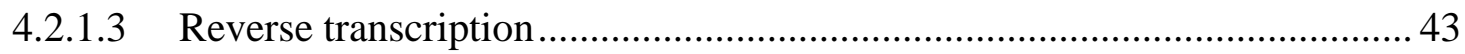

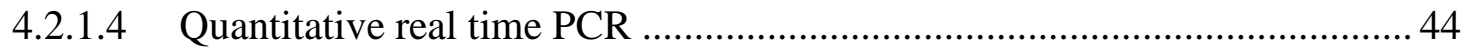

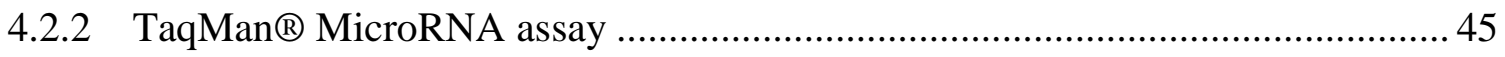

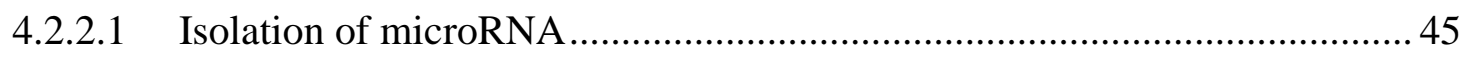

4.2.2.2 MicroRNA specific reverse transcription ............................................. 45

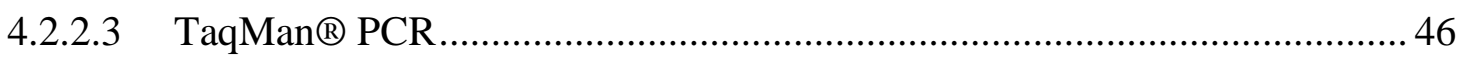

4.2.3 Gene expression analysis using microarray .................................................... 47 


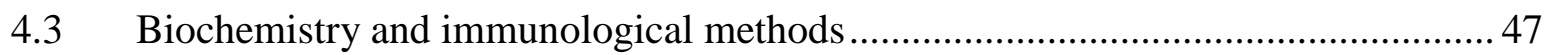

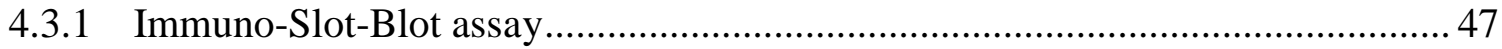

4.3.1.1 Extraction of DNA using NucleoBond ${ }^{\circledR}$ AXG columns ............................ 48

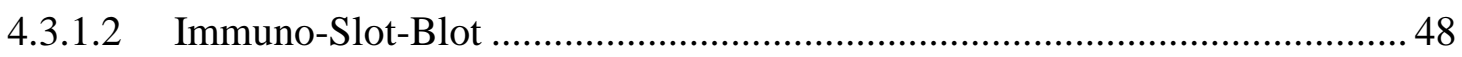

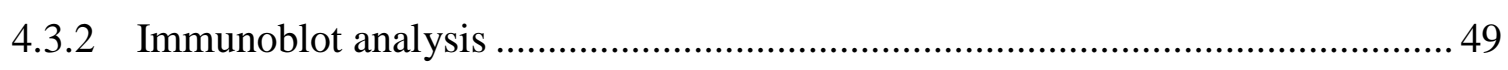

4.3.2.1 Generation of cell lysates for SDS-PAGE analysis ................................... 49

4.3.2.2 Determination of protein concentrations ................................................ 49

4.3.2.3 Separation of proteins by SDS-PAGE ....................................................... 49

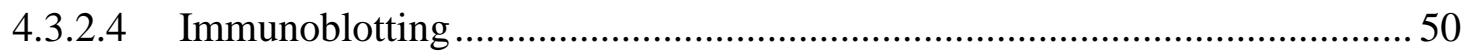

4.4 MSP RAS injection in mice and HSP90 inhibition............................................... 51

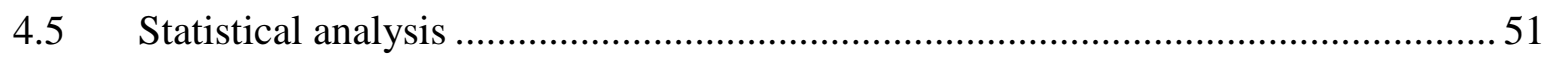

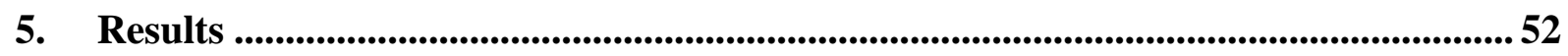

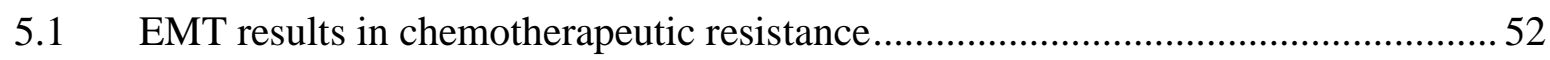

5.1.1 HMLE (RAS) system as a convenient model for EMT ….................................52

5.1.2 In response to Cisplatin no apoptosis is detectable in MSP and MSP RAS cells 54

5.1.3 MSP RAS cells display a general apoptosis deficit ............................................57

5.2 Bcl-xL overexpression protects MSP RAS cells from chemotherapy ..................... 59

5.2.1 EMT causes overexpression of the anti-apoptotic protein Bcl-xL.......................59

5.2.2 Bcl-xL siRNA knock down sensitizes MSP RAS cells to apoptosis induction ... 61

5.2.3 Bcl-xL protein level are increased in disseminated tumor cells in human tissue 62

5.2.4 Bcl-xL level in MSP RAS cells are not detectably regulated by translation,

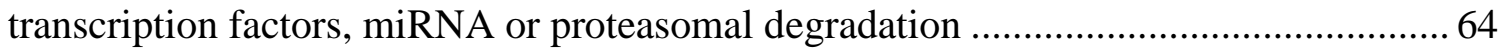

5.3 Overcoming the chemoresistance of MSP RAS cells by the inhibition and

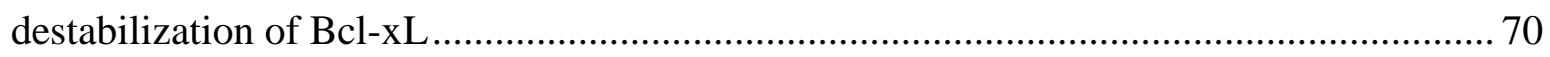

5.3.1 Gossypol stops MSP RAS cell proliferation and induces autophagy ................... 70

5.3.2 HSP90 inhibition destabilizes Bcl-xL and induces apoptosis in MSP RAS cells 72

5.3.3 HSP90 inhibition reduces MSP RAS cell-derived tumors in mice ...................... 74

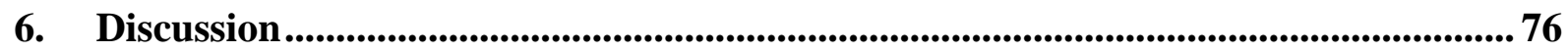

6.1 EMT renders HMLE and HMLE RAS cells resistant to chemotherapy .................. 77

6.1.1 Other examples of EMT correlating with chemoresistance ................................ 77

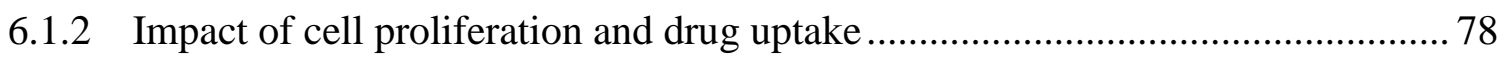

6.1.3 Manipulation of EMT-associated pathways fails to sensitize MSP RAS cells .... 79

6.1.4 MSP RAS cells are resistant to extrinsic apoptosis induction ............................ 80 
6.2 The anti-apoptotic protein Bcl-xL as a mediator of chemoresistance in cancer cells 81

6.2.1 Bcl-xL protects MSP RAS cells from apoptosis

6.2.2 Bcl-xL level in MSP RAS cells are not detectably regulated by miRNAs or candidate transcription factors 82

6.3 Targeting Bcl-xL in MSP RAS cells to overcome chemoresistance ......................... 85

6.3.1 Gossypol induces autophagy in HMLE RAS cells

6.3.2 Bcl-xL destabilization mediated by HSP90 inhibition induced apoptosis in MSP

RAS cells .87

6.3.3 The role of Salinomycin in treating cancer stem cells 89

6.4 Relevance of our findings in human cancer diseases and future aspects .90

6.4.1 Migrating breast cancer cells combine the attributes of EMT and Bcl-xL overexpression. 90

6.4.2 Chromosomal rearrangements lead to $\mathrm{Bcl}-\mathrm{xL}$ overexpression in cancer and embryonic stem cells

6.4.3 Gossypol and HSP90 inhibitors as new strategies to eliminate chemoresistant cells in human cancer therapies

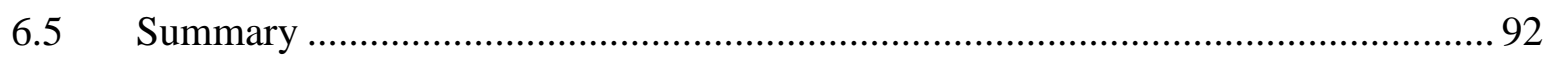

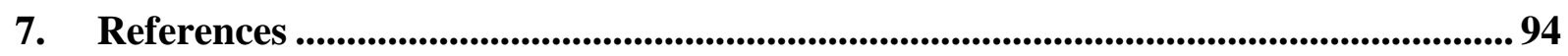

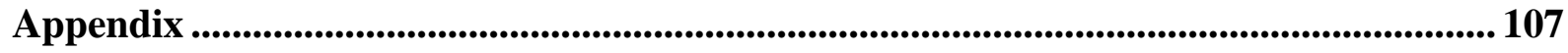

List of differentially regulated genes in MSP cells compared to $24^{+}$HMLE cells. Micro

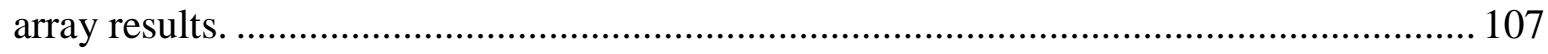

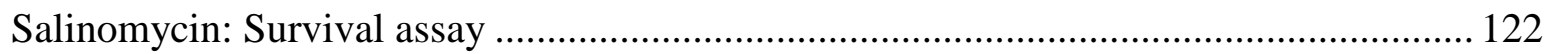




\section{List of figures}

Figure 2.1: Overview of the cytotoxic pathway of Cisplatin.

Figure 2.2: Schematic illustration of the mechanisms involved in chemoresistance in cancer cells.

Figure 2.3: Extrinsic and intrinsic apoptosis signalling pathways. ....................................... 10

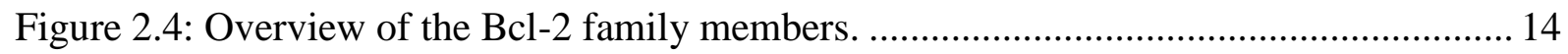

Figure 2.5: The structure of the 5'regulatory sequence of human $\mathrm{Bcl} 2 \mathrm{~L} 1$. ........................... 15

Figure 2.6: Epithelial Mesenchymal Transition (EMT) ................................................... 17

Figure 2.7: Signaling pathways involved in regulation of EMT........................................ 18

Figure 2.8: Double-negative feedback loop of miR-200 and Zeb during EMT...................... 19

Figure 2.9: Stepwise transformation of HMECs to create the HMLE RAS model system. .... 22

Figure 2.10: HMLE RAS cells as model system for EMT. ................................................ 23

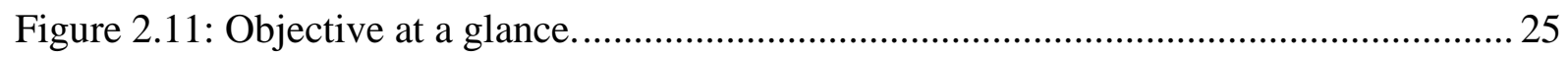

Figure 5.1: Validation of differentially regulated EMT markers in cells before and upon EMT.

Figure 5.2: In response to Cisplatin no induction of apoptosis is detectable in MSP RAS cells.

Figure 5.3: MSP RAS cells recover from Cisplatin treatment. ............................................ 56

Figure 5.4: Cells before and upon EMT display equal amounts of Cisplatin adducts.............56

Figure 5.5: Manipulation of distinct signalling pathways does not sensitize MSP RAS cells

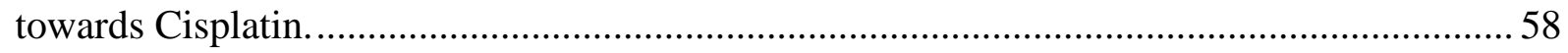

Figure 5.6: MSP RAS are resistant to a variety of chemotherapeutics. ...................................5 58

Figure 5.7: In response to the treatment with death ligands no apoptosis is detectable in MSP

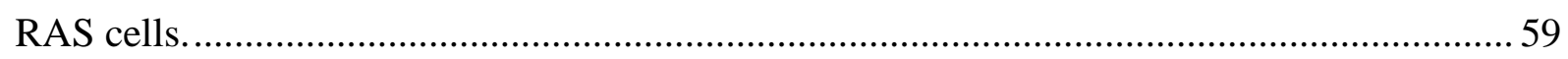

Figure 5.8: EMT results in overexpression of the anti-apoptotic protein Bcl-xL. .................. 60

Figure 5.9: Bcl-xL siRNA knock down sensitizes MSP RAS cells to Cisplatin and Trail

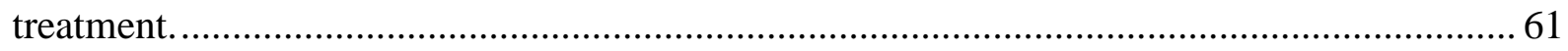

Figure 5.10: Bcl-xL overexpression in 24+HMLE cells results in resistant cells.................... 62

Figure 5.11: Bcl-xL protein levels are increased at the tumor-stroma-interface and in disseminated tumor cells.

Figure 5.12: Bcl-xL overexpression is not detectably regulated by translation or proteasomal degradation. .65

Figure 5.13: Bcl-xL overexpression is not detectably regulated by miRNAs. 66 
Figure 5.14: EMT results in overexpression of the transcription factor cJun, but this is not the reason for Bcl-xL accumulation. 67

Figure 5.15: AP-1 transcription factor knock down has no detectable effect on Bcl-xL level in MSP RAS cells. .68

Figure 5.16: STAT siRNA knock down has no detectable effect on Bcl-xL level in MSP RAS cells 69

Figure 5.17: The Bcl-xL inhibitor Gossypol stops proliferation of MSP RAS cells. 71

Figure 5.18: The Bcl-xL inhibitor Gossypol induces autophagy in MSP RAS cells. .72

Figure 5.19: HSP90 inhibition sensitizes MSP RAS cells towards Cisplatin. .73

Figure 5.20: HSP90 inhibition decreases Bcl-xL mRNA level. 74

Figure 5.21: HSP90 inhibition via systemic 17DMAG treatment induces growth inhibition of MSP RAS cell-induced tumors in mice. .75

Figure 6.1: EMT renders HMLE and HMLE RAS cells resistant to chemotherapy by the overexpression of Bcl-xL. .77

Figure 8.1: MSP RAS cells are sensitive to Salinomycin treatment. (Appendix) 


\section{List of tables}

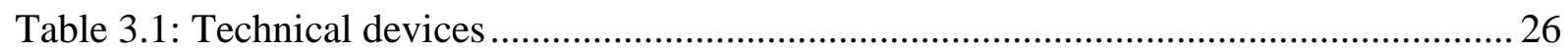

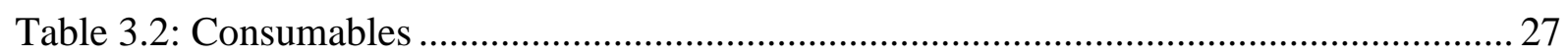

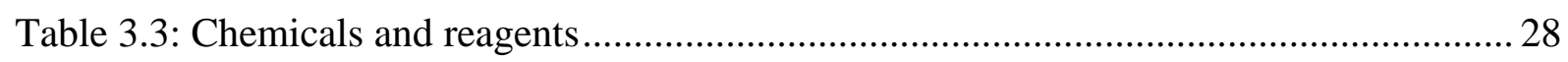

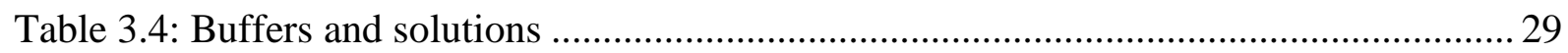

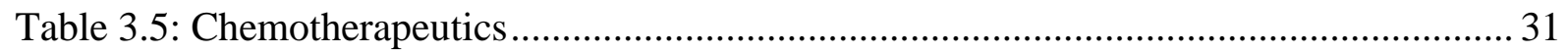

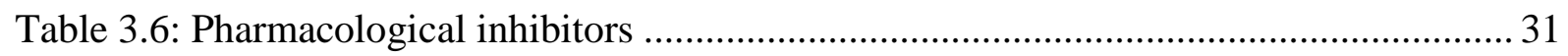

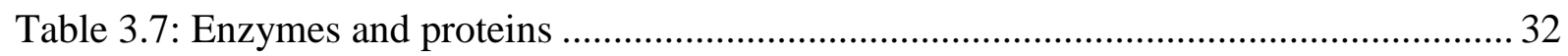

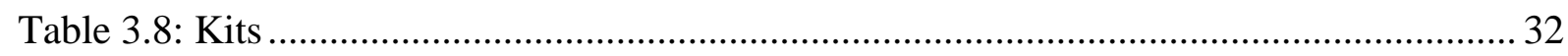

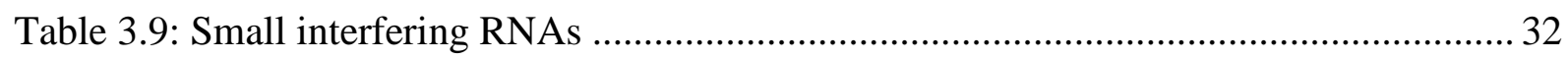

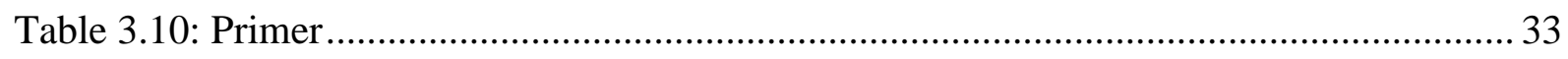

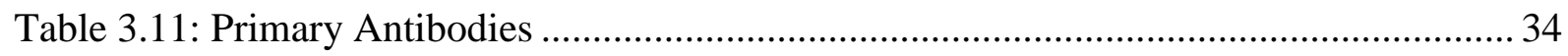

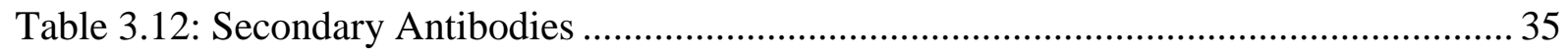

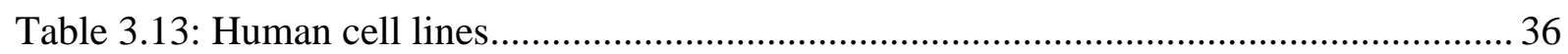

Table 3.14: Contents of MEGM medium for human cell culture (HMLE / HMLE RAS) ....... 36

Table 3.15: Subculture reagents for human cell culture (HMLE / HMLE RAS) .................... 36

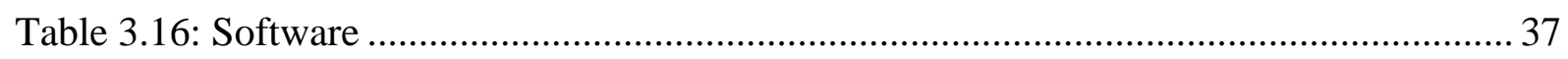

Table 4.1: Concentrations of chemotherapeutics used for cell treatment .............................. 40

Table 4.2: Concentrations of inhibitors for cell treatment .................................................. 41 
Abbreviations

${ }^{\circ} \mathrm{C}$

$\mu \mathrm{g}$

$\mu 1$

$\mu \mathrm{M}$

7-AAD

17AAG

17DMAG

5-FU

$24^{+}$HMLE

3'UTR

5'UTR

A

ABC-transporter

AIF

ALDH 1

AP-1

Apaf-1

APC

APS

ATF

ATP

ATP7A/B

Bax

BCA

Bcl-2

Bcl-xL

Bcl-xS

BH domain

Bid

bp

BPE

BSA

C-terminus

CAD

Caspase

CD

$\mathrm{CDH} 1$

cDNA

CHX
Degree Celcius

Microgram

Microliter

Micromolar

7-Aminoactinomycin

17-N-Allylamino-17-demethoxygeldanamycin

17-Dimethylaminoethylamino-17-demethoxygeldanamycin

5-Fluorouracil

CD24 positive Human Mammary Large T-antigen immortalized Epithelial cells

Three prime untranslated region

Five prime untranslated region

Adenine; Absorbance

ATP binding cassette-transporter

Apoptosis inducing factor

Aldehyde dehydrogenase 1

Activator protein 1

Apoptotic protease activating factor 1

Adenomatous-polyposis-coli

Ammonium persulfate

Activating transcription factor 2

Adenosine triphosphate

Copper-transporting ATPase 1/2

Bcl-2-associated X protein

Bicinchoninic acid

B-cell lymphoma-2

B cell lymphoma-extra large

B cell lymphoma-extra small

Bcl-2 homology domain

$\mathrm{BH} 3$ interacting domain death agonist

Base pair

Bovine pituitary extract

Bovine serum albumine

Carboxy-terminus

Caspase-Activated DNase

Cysteine-dependent aspartate-directed protease

Cluster of differentiation

Cadherin 1 gene

Complementary DNA

Cycloheximide 


\begin{tabular}{|c|c|}
\hline CSC & Cancer stem cell \\
\hline CTC & Circulating tumor cells \\
\hline $\mathrm{Cu}$-transporter & Copper-transporter \\
\hline Су3-СТР & Cy3-Cytidine 5'-triphosphate \\
\hline $\mathrm{d}$ & Day \\
\hline DAB & 3,3'-Diaminobenzidin \\
\hline DD & Death domain \\
\hline DED & Death effector domain \\
\hline DIC & Ductal carcinoma \\
\hline DISC & Death inducing signaling complex \\
\hline DKK & Dickkopf protein \\
\hline DMEM & Dulbecco's Modified Eagle Medium \\
\hline DMSO & Dimethylsulfoxide \\
\hline DNA & Deoxyribonucleic acid \\
\hline dNTP & Deoxynucleotide triphosphate \\
\hline DR & Death receptor \\
\hline dTMP & Deoxythymidine monophosphat \\
\hline DTT & Dithiotreitol \\
\hline dUTP & Deoxyuridine triphosphate \\
\hline E-box & Enhancer box \\
\hline $\mathrm{ECL}$ & Enhanced chemiluminescence \\
\hline EDTA & Ethylene diamine tetraacetic acid \\
\hline eEF2 & Eukaryotic elongation factor 2 \\
\hline e.g. & exempli gratia \\
\hline EGF & Epidermal growth factor \\
\hline EGFR & Epidermal growth factor receptor \\
\hline EGTA & Ethylene glycol tetraacetic acid \\
\hline EMT & Epithelial Mesenchymal Transition \\
\hline Epub & Electronic publication \\
\hline ER & Estrogen receptor \\
\hline ERCC1 & Excision repair cross-complementing protein 1 \\
\hline Erk & Extracellular signal-regulated kinase \\
\hline $\mathrm{EtBr}$ & Ethidium Bromide \\
\hline et al. & et aliale \\
\hline $\mathrm{EtOH}$ & Ethanol \\
\hline Ets & E-twenty six transcription factor \\
\hline FADD & Fas-associating protein with death domain \\
\hline FCS & Fetal calf serum \\
\hline FDR & False discovery rate \\
\hline FGFR & Fibroblast growth factor receptor \\
\hline FOXC1/2 & Forkhead box protein $\mathrm{C} 1 / 2$ \\
\hline G & Guanine \\
\hline g & Gravitational force \\
\hline G1- / G2-phase & Gap1- / Gap2-phase \\
\hline GFP & Green fluorescence protein \\
\hline
\end{tabular}


GSH

GSK3 $\beta$

$\mathrm{h}$

$\mathrm{H}_{2} \mathrm{O}_{2}$

Hif1 $\alpha$

hEGF

HEPES

Her2

hESC

HGFR

HL-60

HMEC

HMLE

HMLE RAS

HNSCC

HR

HRP

HSP70

HSP90

hTERT

HuR

IAP

$\mathrm{IC}_{50}$

ICAD

i.e.

IHC

Inh

IKK

IRES

JAK

JNK

$\mathrm{kDa}$

$\mathrm{M}$

MAP

MAPK

MCF

Mcl-1

MCS

MDA-MB

MDCK

MEGM

MET

$\mathrm{mg}$
Gluthathione

Glycogen synthase kinase $3 \beta$

Hour

Hydrogen peroxide

Hypoxia-inducible factor $1 \alpha$

Human epidermal growth factor

4-(2-hydroxyethyl)-1-piperazineethanesulfonic acid

Human epidermal growth factor receptor 2

Human embryonic stem cell

Hepatocyte growth factor receptor

human leukemic cells

Human Mammary Epithelial cells

Human Mammary Large T-antigen immortalized

Epithelial cells

Human Mammary Large T-Antigen immortalized

Epithelial HaRas expressing cells

Head and neck squamous cell carcinoma

Homologous recombination

Horseradish peroxidase

Heat shock protein of $70 \mathrm{kDa}$

Heat shock protein of $90 \mathrm{kDa}$

human telomerase reverse transcriptase

Hu antigen $\mathrm{R}$

Inhibitor of apoptosis

Half maximal inhibitory concentration

Inhibitor of Caspase-Activated DNase

id est

Immunohistochemistry

Inhibitor

I $\mathrm{B}$ kinase

Internal ribosomal entry site

Januskinase

c-Jun N-terminal kinase

Kilodalton

Molar

Mitogen-activated protein

Mitogen-activated protein kinase

Michigan Cancer Foundation

Induced myeloid leukemia cell differentiation protein-1

Migrating cancer cells

M.D. Anderson-metastatic breast

Madin Darby canine kidney

Mammary Epithelial Cell Growth Medium

Mesenchymal Epithelial Transition

Milligram 


\begin{tabular}{|c|c|}
\hline $\min$ & Minute \\
\hline $\operatorname{miR}$ & micro RNA \\
\hline miRNA & micro RNA \\
\hline MIT & Massachusetts Institute of Technology \\
\hline $\mathrm{ml}$ & Milliliter \\
\hline $\mathrm{mM}$ & Millimolar \\
\hline MMP7 & Matrix metalloproteinase-7 \\
\hline M-MuLV & Moloney-Murine Leukemia Virus \\
\hline mRNA & Messenger RNA \\
\hline MRP & Multi-drug resistance protein \\
\hline MSP & Mesenchymal subpopulation \\
\hline mTOR & mammalian target of rapamycin \\
\hline $\mathrm{n}$ & Sample size \\
\hline $\mathrm{N}$ & Nitrogen \\
\hline $\mathrm{NaAc}$ & Sodium acetate \\
\hline $\mathrm{NCS}$ & Neocarzinostatin \\
\hline NER & Nucleotide excision repair \\
\hline ng & Nanogram \\
\hline $\mathrm{NF \kappa B}$ & $\begin{array}{l}\text { nuclear factor kappa-light-chain-enhancer of activated } \\
\text { B-cells }\end{array}$ \\
\hline $\mathrm{nM}$ & Nanomolar \\
\hline $\mathrm{nm}$ & Nanometer \\
\hline NMUMG & Normal murine mammary gland epithelial \\
\hline NOD/SCID & $\begin{array}{l}\text { Non-Obese Diabetic/Severe Combined Immunodeficien- } \\
\text { cy }\end{array}$ \\
\hline n.s. & Not significant \\
\hline $\mathrm{Nu} / \mathrm{Nu}$ & Nude/Nude \\
\hline $\mathrm{O}^{2-}$ & Superoxide anion \\
\hline $\mathrm{p}$ & Phospho \\
\hline p53 & Tumor protein 53 \\
\hline PARP & Poly (ADP-ribose) polymerase \\
\hline P-glycoprotein & Permeability glycoprotein \\
\hline PBS & Phosphate buffered saline \\
\hline PCR & Polymerase chain reaction \\
\hline PI3-kinase & Phosphatidylinositol 3-kinase \\
\hline $\mathrm{pRB}$ & Retinoblastoma protein \\
\hline PTK & Protein tyrosine kinase \\
\hline $\mathrm{Pt}$ & Platinum \\
\hline qRT-PCR & Quantitative real-time PCR \\
\hline RAS & Rat sarcoma \\
\hline $\mathrm{rcf}$ & Relative centrifugal force \\
\hline RNA & Ribonucleic acid \\
\hline RNAi & Ribonucleic acid interference \\
\hline $\mathrm{rpm}$ & Rounds per minute \\
\hline RT & Room temperature; Reverse transcriptase \\
\hline
\end{tabular}


scr

SD

SDS

SDS-PAGE

sec

sFRP

siRNA

S-phase

SSC

STAT

SV-40

$\mathrm{T}$

TAL

tBid

TBST

TEMED

TGF

TM

TNF

TNF-R

TNS

Trail

Tris

TRK

TS

U

UV

$\mathrm{V}$

VEGF

XIAP

Zeb1/2
Scrambled

Standard deviation

Sodium dodecyl sulfate

SDS-polyacrylamide gel electrophoresis

Second

Secreted frizzled-related protein

Small interfering ribonucleic acid

Synthesis-phase

Saline-sodium citrate

Signal Transducers and Activators of Transcription

Simian-Virus 40

Thymine

Transcriptome analyses laboratory Göttingen

Truncated BH3 interacting domain death agonist

Tris buffered saline + Tween 20

Tetramethylethylenediamine

Tumor growth factor

Transmembrane domain

Tumor necrosis factor

Tumor necrosis factor-receptor

Trypsin neutralizing solution

TNF-related apoptosis-inducing ligand

Trisamine

Tyrosine kinase receptor

Thymidylate synthase

Unit

Ultraviolet

Volt

Vascular Endothelial Growth Factor

$\mathrm{X}$-linked inhibitor of apoptosis

Zinc finger E-box-binding homeobox 1/2 


\section{Abstract}

The developmental program of Epithelial Mesenchymal Transition (EMT) is assumed to be involved in tumor progression, enabling cancer cells to spread from the primary tumor and form metastasis in distant tissues. At least in some cases, disseminated tumor cells display tumor-initiating properties similar to stem cells. In addition, these cells tend to resist chemotherapy. However, it is unclear how cells gain the competency to escape chemotherapeutic treatment upon EMT.

In order to investigate the role of EMT in chemotherapeutic resistance and the underlying mechanisms, we used a HMLE RAS (Human Mammary Large T-Antigen immortalized Epithelial HaRas expressing cell) model system. This EMT system is composed of two different cell lines. On the one hand, the so called $24^{+}$RAS cells display an epithelial morphology and lack efficient migration. On the other hand, the MSP RAS cells (mesenchymal subpopulation) that originate from the $24^{+}$RAS cells have undergone EMT, accompanied by a mesenchymal cell shape and high invasivity. In this study, we applied several chemotherapeutic treatments to both cell lines to assess their response to chemotherapy.

We found that, in contrast to $24^{+}$RAS cells, MSP RAS cells are resistant to the chemotherapeutics Cisplatin, Doxorubicin and Neocarzinostain, but also to the death ligands Trail and $\mathrm{TNF} \alpha$, as seen by reduced PARP and Caspase 3 cleavage upon treatment. Beyond that, we showed that the anti-apoptotic Bcl-2 family member Bcl-xL is overexpressed in MSP RAS cells, in comparison to $24^{+}$RAS, which enables former cells to survive chemotherapy and to continue proliferation after treatment.

We have demonstrated that retrovirus-mediated Bcl-xL overexpression in $24^{+} \mathrm{HMLE}$ renders these cells chemoresistant, as well. Conversely, knocking down Bcl-xL in MSP RAS cells rendered them sensitive to death stimuli. Strikingly, we found Bcl-xL overexpressed in human breast cancer cells at the tumor-stroma-interface, suggesting a general role of Bcl-xL in migrating cancer cells. We found that inhibition of Bcl-xL with the BH3 mimic Gossypol results in chemosensitivity of MSP RAS cells. The same observation was obtained upon Bcl-xL destabilization, mediated by the inhibition of the chaperone HSP90 in vitro and in vivo, demonstrating that we found an option to overcome chemoresistance of cells that have undergone EMT.

In conclusion, our data reveal Bcl-xL as a key mediator of apoptosis-resistance in cells upon EMT. We propose a causal relation between EMT, Bcl-xL overexpression and chemoresistance, and we suggest $\mathrm{Bcl}-\mathrm{xL}$ as a promising target in cancer therapies. 


\section{Introduction}

\subsection{Need for new cancer therapies to eliminate subpopulations of tumor cells}

Cancer represents a major health problem worldwide. Ferlay et al. estimated 3.45 million new cases of cancer in Europe in 2012, with the most frequent cancer type being female breast cancers with about 464.000 cases [1]. The main difficulty in eliminating cancer is the occurrence of metastases that differ from their origin, exhibit increased invasiveness and are inaccessible for conventional chemotherapy causing cancer regrowth. On the contrary, new research has found that chemo- and radiotherapy of primary tumors can encourage the development of metastases [2]. Correspondingly, metastases are associated with a poor prognosis for cancer patients [3]. Hitherto, it was assumed that metastatic cells do not differ from the primary tumor and were therefore not considered for cancer therapy. However, it was recently published that the gene expression patterns of tumor cells that survived chemotherapy differs from cells belonging to the primary tumor, and this particularly involved genes govern cell cycle progression and cell survival. Consistent with these findings, it could be observed that $\mathrm{f}$. e. breast cancers exhibit a subpopulation of cells that contain tumor initiating properties associated with resistance to chemotherapies as compared to the major cell population of the differentiated tumor [4]. Furthermore, conventional therapies targeting the primary tumor indeed result in tumor regression but do not protect from tumor cells causing a relapse associated with a lack of chemosensitivity. The outcome of chemotherapy to metastasis was not considered so far, but understanding this issue will enable clinics to develop new cancer therapies and demand progressive research in the field of chemotherapeutic resistance [5].

\subsection{Chemotherapy}

Nowadays, one of the most prominent medical challenges is cancer therapy. While surgery and irradiation are specifically applied to solid tumors, their combination with chemotherapy is essential to eliminate disseminated tumors and metastasis. Since cancer cells are predominantly fast replicating cells, chemotherapeutics often target the cell cycle and inhibit cell growth and reproduce [6]. Chemotherapy can directly affect cell division by disabling the mitotic spindle, like the taxanes or inhibit growth signalling pathways. However, the most notably used chemotherapeutics are agents that cause DNA damage and therefore prevent cell cycle progression and induce cell death [7]. Lesions that occur during the S-phase of the cell cy- 
cle can additionally disable replication fork progression followed by the generation of DNA double-strand breaks that mainly result in the induction of programmed cell death in cancer cells [7].

Anti-tumor agents can be classified into several groups according to their mechanism of action [8]. The first group contains nucleoside analogues including the antimetabolites Gemcitabine and 5-Fluorouracil (5-FU). These compounds interfere specifically with the S-phase of the cell cycle and provoke the termination of DNA replication. The second group comprises enzyme inhibitors. These anti-tumor agents specifically act on enzymes, which are involved in DNA replication. On the one side, there are DNA methyltransferase inhibitors such as $2^{\prime}$-deoxy-5-azacytidine that result in missing DNA methylation and therefore evoke the activation of silenced genes, including tumor-suppressor genes. On the other side, anthracyclines like Doxorubicin and Daunorubicin induce double-strand breaks by the inhibition of topoisomerase II, an enzyme that relaxes supercoiled DNA by cleavage and religation [9; 10]. Additionally, anthracyclines are able to intercalate in the DNA and thereby stabilize the DNA duplex and prevents the helicase from separating the strands [11]. The last group of chemotherapeutics directly damages the DNA resulting in the induction of DNA adducts, DNA strand breaks and DNA-protein cross links which immediately trigger the DNA damage response [7]. Alkylating agents, like Cyclophosphamide, attach alkyl groups to the DNA and thereby inhibit DNA replication, while platinum agents induce DNA crosslinks and DNA adducts which causes the inhibition of DNA replication and transcription. Finally the radiomimetic DNA cleaving agents like Neocarzinostatin (NCS) evoke single- and double-strand breaks.

Unfortunately, chemotherapeutics impact healthy cells as well, especially tissues with high turnover rates such as the bone marrow or the epithelium of the gastrointestinal tract. The application of chemotherapeutic combinations attempts to minimize harmful secondary effects of chemotherapy while coincidentally increasing its anti-tumor-activity [6].

\subsubsection{Particularized view on chemotherapy used in this thesis}

\subsubsection{Platinating agents}

This group of chemotherapeutics includes the platinum based chemotherapeutic drugs Cisplatin, Carboplatin and Oxaliplatin. These agents are used for a wide range of solid tumors and represent an effective therapy for head, neck, testicular, ovarian, bladder, prostate and lung cancer inducing DNA lesions and therefore inhibiting DNA replication [12]. 
The anti-tumor activity of Cisplatin was first described by Rosenberg et al. in 1969 [13]. Cisplatin is a cytostatic agent which contains a complex bonded platinum atom. Due to the low chloride concentrations present in the cytoplasm, the platinum complex becomes activated by the replacement of chloro-ligands in to water molecules. Thereby, the molecule obtaines a positive charge and initiates the formation of mono-adducts between the platinum atom and DNA, particularly at the nucleophilic $\mathrm{N}$-atoms of the purines, which induce bivalent intraand interstrand cross links $[14 ; 15]$. DNA replication stops and strand breaks are evoked resulting in a G2-phase arrest of the cell cycle and the initiation of DNA damage repair or programmed cell death (Figure 2.1) [16].

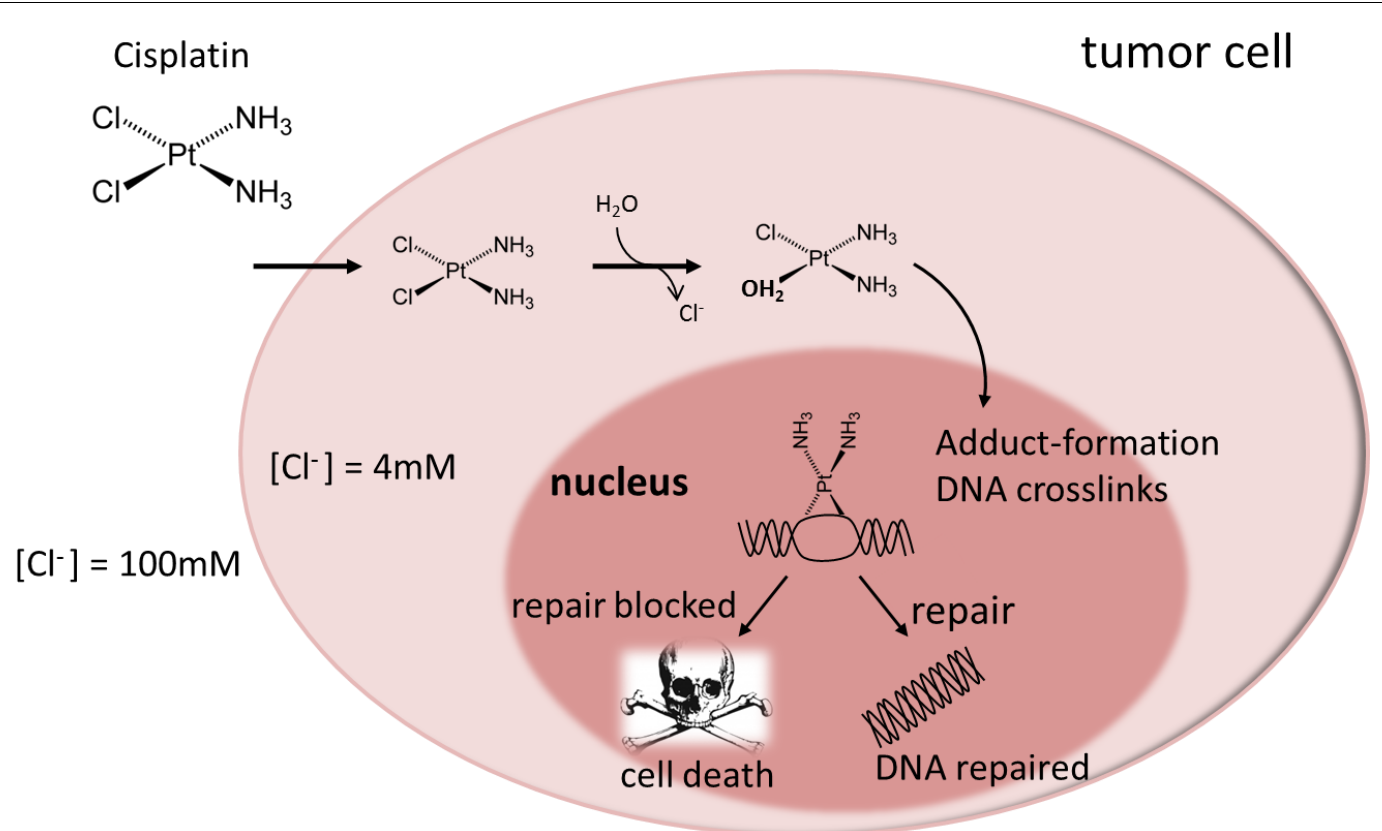

Figure 2.1: Overview of the cytotoxic pathway of Cisplatin.

Cisplatin translocates into the cell and is activated by replacing the chloro-ligands into water molecules followed by binding to DNA. Therefore Cisplatin induces intra- and interstrand crosslinks. The DNA lesions are either repaired or programmed cell death is induced. Referring to Alderden et al. [17].

To overcome the resistance that Cisplatin treated tumors could develop, either during therapy cycles, as was observed for ovarian cancer, or by the acquisition of intrinsic resistance, as observed for prostate, colorectal, lung or breast cancer patients, thousands of Cisplatin analogues were synthesized. These "second- and third-generation" platin drugs, such as Carboplatin, which lacks nephrotoxicity, is less toxic to the gastrointestinal tract and less neurotoxic compared to Cisplatin have receive worldwide approval and clinical use [18; 19]. In contrast to Cisplatin, Carboplatin contains cyclobutane-1,1-dicarboxylate-ligands which mediates good aqueous solubility and greater stability because of the six-membered ring with a 
platinum ion. These properties illustrate that Carboplatin displays a spectrum of activities comparable to Cisplatin but is better tolerated and therefore predominantly clinically used [19]. Interestingly, phase II trials for metastatic breast cancers have shown that Carboplatin alone produced objective response rates of $20-35 \%$ [20; 21]. Additionally, synergistic antitumor efficacies were observed in phase II and III trials for a combination therapy with Paclitaxel or Trastuzumab, favoring Carboplatin as a standard active substance for cancertherapies for metastatic breast cancer, especially for patients that fail to respond to anthracycline-based therapy $[22 ; 23 ; 24 ; 25]$.

\subsubsection{Anthracyclines}

Anthracyclines were first described in the 1960's by Brockmann et al. and are derived from Streptomyces peucetius. They possess anti-tumor activity against breast cancer, sarcoma and lymphoma $[9 ; 26]$. Anthracyclines are red aromatic polyketides exhibiting a heterogeneous structure due to distinctive aglyconic and sugar residues. The most prominent agents in this group are Doxorubicin and Daunorubicin, followed by their derivatives Epirubicin and Idarubicin, respectively. As reviewed by Gewirtz, anthracyclines display a wide range of cellular interventions. First anthracyclines induce adduct formation and cross linking of the DNA as well as the induction of DNA damage through interference with topoisomerase II. Furthermore, anthracyclines form a ternary complex with DNA and topoisomerase II which induces DNA single- and double-strand breaks, followed by G1- or G2-arrest. Moreover, the incurrence of reactive oxygen species as superoxide anion $\left(\mathrm{O}^{2-}\right)$ and hydrogen peroxide $\left(\mathrm{H}_{2} \mathrm{O}_{2}\right)$ during the biotransformation of anthracyclines results in oxidative damage of the DNA as well as the cell membranes [9]. Additional studies have shown that anthracyclines interfere with macromolecular biosynthesis and therefore inhibit DNA synthesis in the tumor cell. Anthracyclines are able to intercalate in DNA and also disable the DNA polymerase [27]. In the end, a clear consequence of treatment with anthracyclines is damaged DNA triggering the DNA damage response and subsequent induction of programmed cell death [9; 27].

\subsubsection{Neocarzinostatin}

Neocarzinostatin (NCS) is an antibiotic from Streptomyces carzinostaticus that has anti-tumor activity. The acidic polypeptide is cross-linked with two disulfide bridges and consists of 109 amino acid residues. Hence NCS belongs to the family of macromolecular antibiotics with the ability to inhibit DNA synthesis and induce DNA damage [28; 29]. The biological activity of NCS is mediated by a non-covalently bound chromophore which binds to DNA in a two-step process. It first binds externally to a specific DNA sequence, followed by the intercalation of 
the chromophore between adjacent DNA base pairs. The latter induces single- and doublestrand breaks particularly in A-T rich regions $[30 ; 31]$. In the 1980 's, clinical trials were performed in Japan testing NCS for chemotherapeutical use. Unfortunately various types of toxic manifestations have been demonstrated whereby NCS could not gain acceptance for clinical use $[32 ; 33]$. However, as a radiomimetic DNA cleaving agent, NCS is a valuable agent for laboratory use.

\subsubsection{Chemoresistance and mechanisms involved}

The efficacy of chemotherapy is limited by the acquisition of chemoresistance in cancer cells. Thereby tumors are either intrinsically resistant to chemotherapy prior to treatment, or tumors that were originally chemosensitive gain resistance during cancer therapy. Most notably, chemoresistance does not only concern the anti-tumor agents that were initially used; rather tumors become cross-resistant to other chemotherapeutics with a different mode of action as well [34]. Another aspect of ineffectiveness of chemotherapeutic treatment involves the survival of a population of tumor cells during treatment that subsequently initiate the cancer regrowth upon termination of treatment [35]. Drug-resistance includes various factors that affect chemo-response and can occur at several levels (Figure 2.2). On the one hand, there are mechanisms that limit the doses of chemotherapy reaching the tumor or affect the tumor microenvironment. On the other hand, cancer cell specific mechanisms that are pointed out in the following sections include drug transport into the cells, drug inactivation, alteration of the drug target and processing drug-induced damage and finally the prevention of apoptosis [34].

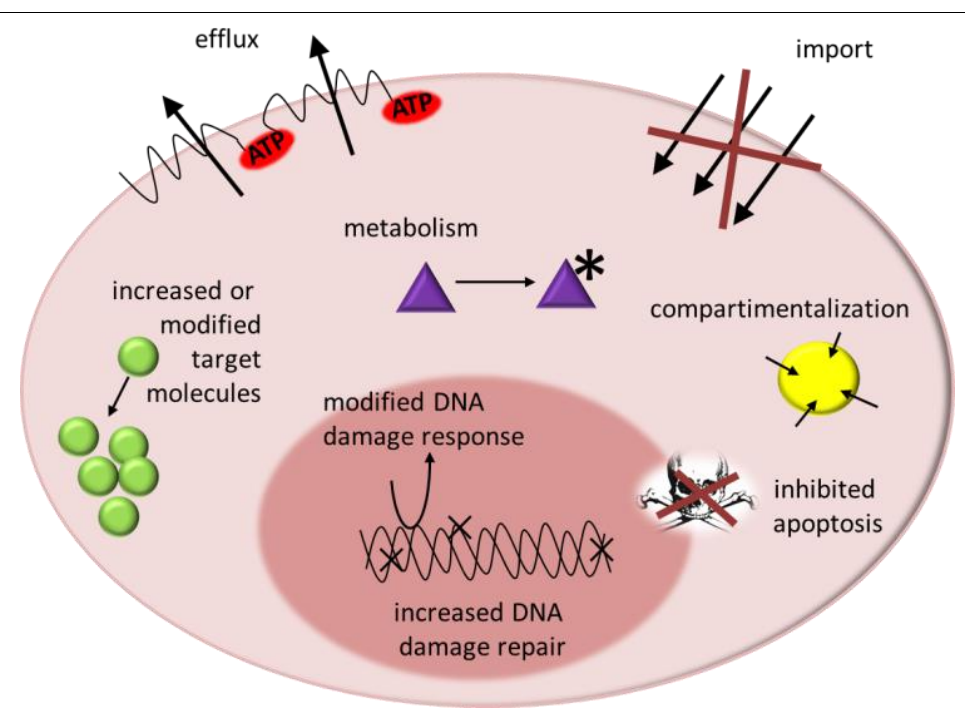

Figure 2.2: Schematic illustration of the mechanisms involved in chemoresistance in cancer cells.

Legend on the next page. 
Figure 2.2: Schematic illustration of the mechanisms involved in chemoresistance in cancer cells. Efflux and import occur by means of membrane-associated transporters including ABC-transporter and multi-drug resistance protein (MRP) family members. Referring to Gottesman [36].

\subsubsection{Drug influx and efflux}

Altered drug influx and efflux is often involved in enhanced chemoresistance. In particular, it is well known that changes in the expression of ABC-transporters, especially the $\mathrm{P}$ glycoprotein and the multi-drug resistance protein (MRP) family, are involved in increased efflux of chemotherapeutic drugs [34]. Hydrophobic anti-tumor agents, including taxanes and anthracyclines, are actively transported out of the cell by these proteins [34]. For Cisplatin, it is known that the $\mathrm{Cu}$-transporter ATP7A and 7B are connected to its export. Thereby, ectopic expression of ATP7B results in diminished Cisplatin sensitivity because of a decrease in Cisplatin accumulation. Interestingly, the ectopic expression of ATP7A evokes the accumulation of Cisplatin in endosomes inhibiting its effect on DNA [37]. However, Cisplatin has not been described as a substrate for the P-glycoprotein and therefore not carried out by these efflux pumps [15].

\subsubsection{Drug inactivation}

Furthermore drug inactivation has been shown to play an important role in chemoresistance, which reduces the amount of anti-tumor agent that is available to reach their targets in cancer cells [34]. It has been shown that the activated platinating agents, including Cisplatin, Carboplatin and Oxaliplatin are able to react with thiol-containing molecules, like glutathione (GSH) and metallothioneins in the cytoplasm. Glutathione is an anti-oxidant in the body that prevents oxidative stress. Binding of Cisplatin to Glutathione results in adduct formation associated with detoxification of Cisplatin which either traps Cisplatin in the cell or results in an increased efflux of Cisplatin by unidentified organic anion pumps [38; 39]. Another aspect of missing drug activation, reflects the fact that several chemotherapeutics have to be metabolized into an active form in the body. The topoisomerase I inhibitor Camptothecin, for example, is metabolized by a carboxylesterase, which is found to be less active in cancer cells [40].

\subsubsection{Drug targets and regulatory proteins}

Generally, the mutation of chemotherapeutic targets or alteration of their expression levels has a substantial impact on drug resistance. Lang et al. have shown that altered topoisomerase II expression or activity results in drug resistance against topoisomerase II inhibitors and cross-resistance to anthracyclines like Doxorubicin [41]. Additionally, the thymidylate synthase (TS), which is an essential enzyme for the biosynthesis of dTMP from Uracil and indis- 
pensable for DNA repair and replication, is an important target for chemotherapy. TS is inhibited by 5-FU; correspondingly high levels of TS are associated with resistance to 5-FU [42]. Importantly, besides alterations in the expression of drug targets, alterations in the expression of oncogenes like $c$-fos and $c$-jun and tumor suppressors, such as $p 53$, are also involved in cellular resistance. The increased expression of $c$-fos and $c$-jun for example is found to be associated with Cisplatin-resistant cells while the down-regulation of these genes reverses resistance to Cisplatin. Importantly, Cisplatin treatment induces $c$-fos and $c$-jun expression, which results in increased expression of genes that contain binding sites for the transcription factor AP-1 (activator protein 1). AP-1 is a transcription factor complex, which consists of Fos and Jun heterodimers. AP-1 regulated genes includes $c$-myc and metallothioneins that are involved in cell proliferation and in the end cause chemoresistance [15].

\subsubsection{DNA damage repair}

DNA damage caused by chemotherapy is either induced directly, e.g. by platinum agents, or indirectly, as it is known for 5-FU and topoisomerase inhibitors. Induction of DNA damage is followed by DNA repair and in the case of extensive damage by programmed cell death. The efficiency of cancer cells to repair DNA can determine their resistance to chemotherapy [34]. For example, adducts that were formed by Cisplatin and DNA are repaired by nucleotide excision repair (NER) and homologous recombination. Cells that are deficient in NER are more sensitive to Cisplatin than the wildtype cells. However, restoration of NER activity is accompanied by a decrease in drug sensitivity. Moreover, it has been published that Cisplatin treatment leads to increased levels of the excision repair cross-complementing 1 protein (ERCC1) which is a rate-limiting factor of the NER system and is associated with Cisplatin resistance $[15 ; 34]$. On the other hand, several studies demonstrated that Cisplatin resistance is accompanied by adduct tolerance and an increase in the ability to replicate past Cisplatin adducts $[43 ; 44]$.

\subsubsection{Inhibited apoptosis}

Treatment of tumor cells with chemotherapy involves a lot of different signalling pathways acting together. As mentioned above a major chemotherapeutic activity is the induction of DNA damage followed by apoptosis. Since tumor cells exhibit modifications in the induction of apoptosis following chemotherapy, this is an important issue with regard to chemotherapeutic resistance. Failure to induce apoptosis can be reasoned by overexpression of antiapoptotic proteins like Bcl-2 or the decreased expression of pro-apoptotic proteins, such as the death receptor Fas [45]. Apoptosis can be regulated by an intrinsic signaling pathway mediat- 
ed via the mitochondria, or extrinsically via activation of the death receptors. In cancer cells both apoptosis inducing pathways can be disrupted leading to a failure to undergo apoptosis after chemotherapeutic treatment. Many solid tumor cells exhibit increased expression of the protein Bcl-2, preventing activation of the caspase cascade downstream of the mitochondria. On the other hand, down-regulation of the anti-apoptotic proteins including Bcl-xL is associated with chemosensitivity [46; 47]. It was also observed that Cisplatin-resistant cells display reduced activation of the JNK signalling pathway. This pathway activates apoptosis by modulating the activities of mitochondrial pro- and anti-apoptotic proteins through their phosphorylation state [48]. Cisplatin-resistant ovarian carcinoma and lymphoma cells were especially found to exhibit no JNK activation [15; 49]. Additionally, in contrast to apoptosis inhibition, cancer cells show continuous activation of pro-survival pathways as well. For example, the protein tyrosine kinases (PTKs) regulate the anti-apoptotic signaling pathways that have been described to be highly over expressed in tumor cells. A well-known family of the PTKs is the epidermal growth receptor (EGFR) family which includes EGFR and Her2. The binding of growth factors such as EGF or TGF $\alpha$ to these receptors leads to the activity of pro-survival and proliferative pathways [47]. The activation of the PI3-kinase/Akt and STAT pathways are especially known to be highly induced in tumors such as breast, head and neck cancer, respectively [50]. Moreover, the overexpression of EGFR and Her2 is accompanied by chemoresistance and a poor prognosis for cancer patients. $15-25 \%$ of breast cancers contain high expression levels of Her2. To block Her2, the specific antibody Trastuzumab that targets membrane bound Her2 is clinically used in combination with chemotherapy, resulting in increased response rates [47].

\subsection{Apoptosis}

Apoptosis is an important kind of programmed cell death; it is a genetically determined mechanism by which cells are eliminated, that normally occurs during development, maintenance of homeostasis and to eliminate cells that can be threat to the organism, such as virusinjected or tumor cells [51]. The term of apoptosis was firstly described by Kerr et al. in 1972 [52] based on morphological cell changes and formation of "apoptotic bodies". However, the phenomenon itself was observed 100 years before by Carl Vogt in 1842 [53]. Apoptosis is characterised by a series of morphological and biochemical changes that are strictly determined. Apoptotic cells typically lose contact with neighbouring cells, show deformation and lose cell volume. Apoptosis is accompanied by nuclear condensation, chromatin aggregation and finally endonucleolytic DNA fragmentation into multiples of 185 base pairs. Subsequent- 
ly, the membrane potential of the mitochondria collapses and $\mathrm{pH}$-values decrease. Upon DNA fragmentation the cell decomposes by mouldering into apoptotic bodies which contain cytosol, the condensed chromatin and organelles. The apoptotic bodies are phagocytised by macrophages and thus removed from the tissue, preventing the release of inflammatory cytokines $[54 ; 55]$. The point of no return during apoptosis is characteristically marked by the proteolytic cleavage of PARP and is correspondingly a hallmark of apoptosis. PARP is usually activated by DNA strand breaks to stabilize those and the chromatin. However, if the DNA lesions are not repairable the cell induces irreversible apoptosis [56; 57].

Apoptosis induction can be divided into two main pathways: On the one hand, the intrinsic mitochondria-mediated induction of apoptosis that is triggered by cellular stress, chemotherapy and irradiation and is dependent on intracellular checkpoint proteins [55]. On the other hand, the extrinsic death receptor-induced pathway mediated for example by Fas or Trail receptors. Apoptosis induction frequently leads to the activation of several signalling pathways that shift the balance from anti- to pro-apoptotic proteins associated with cell cycle arrest and activation of the caspase cascade (Figure 2.3) [54].

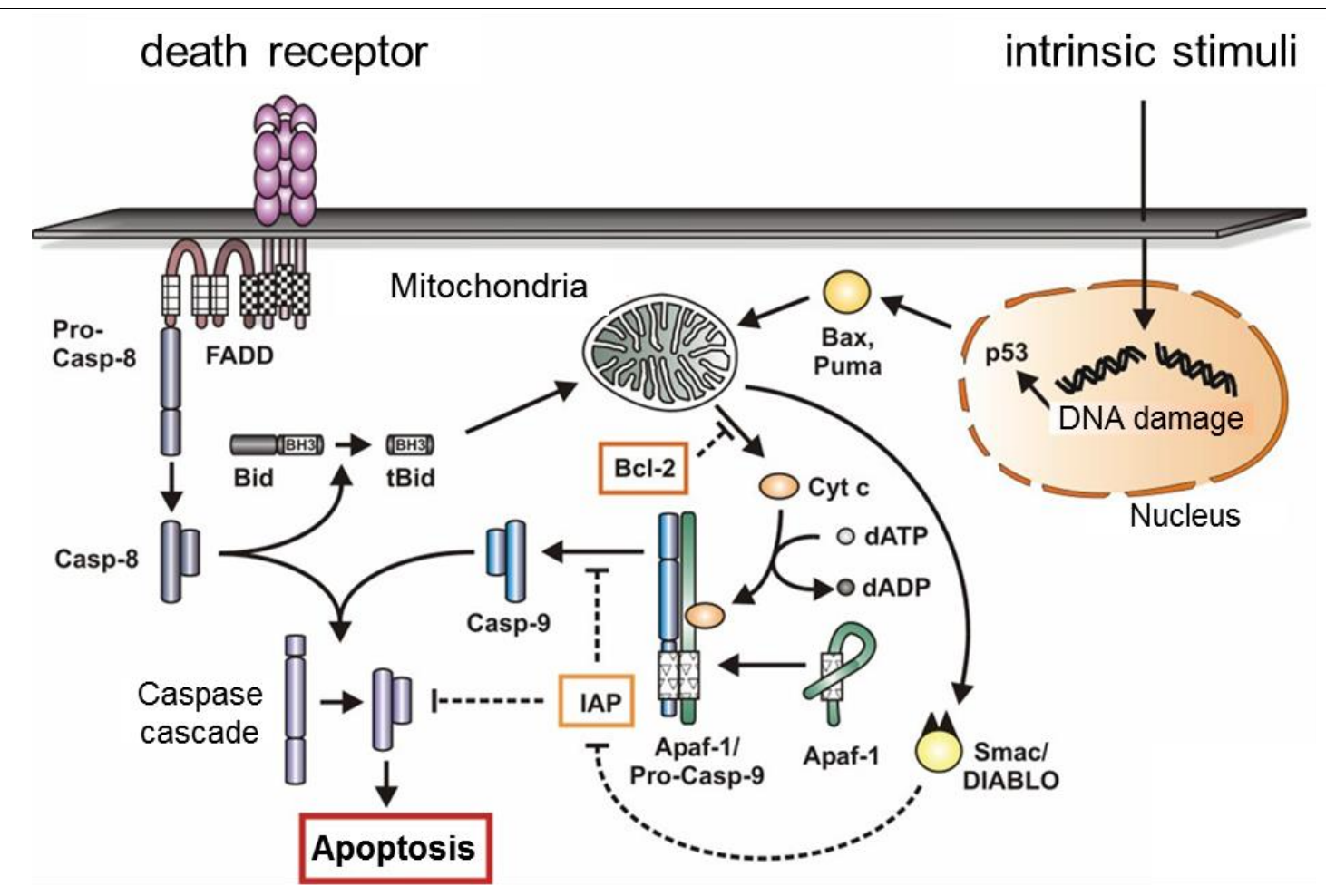

Figure 2.3: Extrinsic and intrinsic apoptosis signalling pathways.

Extrinsic apoptosis pathway: Death ligands bind to the death receptors which causes their oligomerisation. Subsequently, the adaptor molecule FADD (fas-associating protein with death domain) is recruited to the intracellular death domain (DD) of the receptors forming the death inducing signaling complex (DISC) which results in the recruitment and processing of pro-caspase 8 . Caspase 8 either directly induces programmed cell death or cleaves the $\mathrm{BH} 3$ only protein Bid. Truncated Bid (tBid) translocates to the mitochondria induces cytochrome $\mathrm{c}$ release and therefore activates Caspase 3 dependent cell death. tBid connects the two apoptosis pathways. Intrin- 
sic apoptosis pathway: Cellular stress leads to the interaction of the Bcl-2 family members Bax and Puma with the mitochondrial outer membrane causing permeabilization and release of cytochrome c. Cytochrome $\mathrm{c}$ acts as secondary messenger forming the apoptosome with Apaf-1 and pro-caspase 9. Caspase 9 is processed and subsequently activates Caspase 3 and 6 initiating the caspase cascade. The mitochondria membrane permeabilization is inhibited by anti-apoptotic members of the Bcl-2 family. Moreover the pro-apoptotic factor Smac/Diabolo that is released from the mitochondria at later stages of the programmed cell death inhibits the IAPs (inhibitors of apoptosis) supporting apoptosis induction. Adapted from K. Lauber, Dissertation at the University of Tübingen [58].

There are three different mechanisms characterized by which a cell gains resistance to apoptosis induction. On the one hand, the disruption of the balance between pro-and anti-apoptotic proteins which is regulated by several proteins including p53 but also oestrogens in the breast epithelium. At that, it is known that oestrogens are able to induce anti-apoptotic Bcl-2-family members in breast epithelial cells as well as in several breast cancer cell lines causing prolonged survival of these cells $[59 ; 60 ; 61]$. On the other hand, the reduction of caspase activity and finally the absence of death receptor signalling play an important role in the acquisition of apoptosis-resistance [61].

\subsubsection{Caspases}

Caspases are central initiators and executioners of apoptosis [54]. Caspases are cysteinedependent aspartate-directed proteases that usually occur in the cell as inactive pro-enzymes. Once activated they can trigger other caspases and lead to the amplification of the apoptotic signalling pathway [62]. Caspases are synthesized as pro-caspases containing a pro-domain on their N-terminus, followed by a large and small subunit. During caspase activation the prodomains are cleaved off by other caspases and the small and large subunits are separated behind the aspartate residues by a proteolytical process [53]. In the end, the active caspases are formed by heterotetramers consisting of two small and two large subunits [54; 62]. Overall 14 mammalian caspases have been found and can be divided into three functional groups: the initiator caspases, including Caspase 2, 8, 9 and 10 characterized by long pro-domains; the effector caspases-, such as Caspase 3, 6 and 7 containing short pro-domains; and finally inflammatory caspases involved in cytokine activation named Caspase 1, 4, 5, 11, 12 and 13 $[53 ; 62]$.

The most important effector caspase is Caspase 3, which is activated by any of the initiator caspases and is able to activate the endonuclease Caspase-Activated DNase (CAD). Generally, CAD is complexed with its inhibitor forming ICAD. However, in apoptotic cells activated Caspase 3 cleaves ICAD and releases CAD that subsequently degrades the chromosmal DNA [51]. Moreover, effector caspases cleave specific protein substrates, including the procaspases themselves to amplify the death signal [54]. 
In mammals, several proteins act as inhibitors for caspases belonging to the inhibitor of apoptosis (IAP) family such as XIAP and survivin [62]. IAPs conduct their function through direct interaction with pro-caspase 9 associated with inhibition of its processing, competing for Apaf-1 binding instead of Caspase 9 and the direct inhibition of active caspases [61].

The extrinsic and intrinsic pathways of apoptosis are associated and affect each other while coming together at the same point where Caspase 3 and Caspase 7 are activated followed by cell shrinkage, chromatin condensation and DNA fragmentation and finally degradation of nuclear and cytosolic proteins [62].

\subsubsection{Extrinsic apoptosis induction}

The induction of the extrinsic apoptotic signalling pathway is triggered by the binding of extracellular death ligands to the plasma membrane associated death receptors. The death receptors belong to the tumor necrosis factor receptor (TNF-R) superfamily including DR4, DR5 TNF-R1 and Fas [63]. The specific binding of the death ligands is mediated by a cysteine rich extracellular subdomain that trimerizes upon ligand binding and results in the activation of the respective receptor. The intracellular part of the death receptors contains a death domain (DD) that recruits adaptor molecules, such as FADD (Fas-associating death domain), upon ligand binding. The adaptor molecules themselves additionally contain a so called death effector domain (DED) which associates with pro-caspase 8, all together forming the death-inducing signalling complex (DISC) [62]. The formation of DISC results in the autocatalytic activation of Caspase 8 which then processes downstream effector caspases ending up in programmed cell death [54]. Cells that induce this direct and caspase-dependant pathway are also described as type I cells [64]. In contrast, there are also type II cells that are not able to induce a signalling caspase cascade that is strong enough to result in cell death. Alternatively, these type II cells make use of activation of the mitochondria-mediated apoptotic signalling pathway. In this case Caspase 8 is also able to cleave the $\mathrm{BH} 3$ only protein Bid. The truncated form of $\mathrm{Bid}, \mathrm{tBid}$, translocates to the mitochondria where it induces the release of cytochrome $\mathrm{c}$ and evokes the activation of Caspase 9 and Caspase 3, ultimately resulting in cell death [53].

\subsubsection{Intrinsic apoptosis induction}

The pivotal mediator of the intrinsic apoptotic signalling pathway is the mitochondrion. The activation of intrinsic apoptosis takes place by cellular stress including DNA damage and oxidative stress, but also cytostatic agents and irradiation. Consequently, levels of pro-apoptotic proteins, such as Bax increase, followed by the permeabilization of the mitochondrial mem- 
brane and release of pro-apoptotic factors, such as cytochrome c, that in the end triggers the caspase cascade $[54 ; 65]$.

A major event during the induction of intrinsic apoptosis is the disruption of the mitochondrial inner transmembrane potential and the permeability transition. This means the abrupt permeabilization of the inner mitochondrial membrane which results in the release of proapoptotic factors into the cytoplasm [54]. Therein, the pro- and anti-apoptotic members of the Bcl-2 family are involved. While the pro-apoptotic Bcl-2 family proteins promote the release of cytochrome c, anti-apoptotic members cause its inhibition [61]. Upon receiving the death signal, the pro-apoptotic Bcl-2 family members undergo a conformational change and subsequently target and integrate in the mitochondrial outer membrane [66]. Integration into the mitochondrial membrane contributes to formation of proteolipid pores which in turn results in the loss of membrane integrity and lead to the release of two main groups of pro-apoptotic factors from the intermembrane space into the cytoplasm. The first group contains cytochrome c, Smac /Diabolo and the serine protease HtrA2/Omi. These proteins activate the caspase-dependant mitochondrial pathway [51]. In the cytoplasm, cytochrome c acts as a secondary messenger and supports the conformational change of Apaf-1. This results in the building of the apoptosome together with pro-caspase 9. This interaction enables the maturation of pro-caspase 9 to Caspase 9 which is executed by a two-step cleavage event as described above, followed by the activation of the effector caspases, Caspase 3 and Caspase 7 . In contrast, Smac/Diabolo and HtrA2/Omi act as inhibitors for IAPs [53]. The second group of pro-apoptotic factors released from the mitochondria are AIF (apoptosis inducing factor), endonuclease $\mathrm{G}$ and $\mathrm{CAD}$ occurring after the cell has committed to die. AIF causes fragmentation of the DNA and chromatin condensation. Together with endonuclease G, they acting independent of caspases [51]. The strict control on induction of the intrinsic apoptotic signalling pathway is subjected to the interplay of pro- and anti-apoptotic members of the Bcl-2 family which is pointed out in the next paragraph [67].

\subsubsection{Bcl-2 family}

Bcl-2 was initially found to be a proto-oncogene which plays an important role in follicular bcell lymphoma. But later on it was discovered that at least 19 Bcl-2 family members are present in mammalian cells balancing the induction of apoptosis [67]. These members contain at least one of the four conserved Bcl-2 homology $(\mathrm{BH})$ domains designated $\mathrm{BH} 1, \mathrm{BH} 2, \mathrm{BH} 3$ and BH4 (Figure 2.4) [66]. According to their function and structure, the Bcl-2 family members can be divided into three classes: First are the anti-apoptotic proteins including Bcl-2, 
Bcl-xL, Mcl-1 and Bcl-W. These members contain all four BH domains and are initially integral membrane proteins. They are present in the membranes of the mitochondria, endoplasmatic reticulum or the nucleus and maintain the integrity of the mitochondria. Second are the pro-apoptotic proteins Bak and Bax which are able to induce mitochondria outer membrane permeabilization [66]. These apoptotic proteins contain $\mathrm{BH} 1, \mathrm{BH} 2$ and $\mathrm{BH} 3$ but not $\mathrm{BH} 4$. In the third category are the "BH3 only" pro-apoptotic members, which include Bid, Bim, Bad, Bik, Bmf, Bnip3, Hrk, Noxa and Puma. These members share sequence homology only within the $\mathrm{BH} 3$ domain and the fact that the amphipatic $\alpha$-helical $\mathrm{BH} 3$ domain contains a critical death domain $[66 ; 67]$.

The anti-apoptotic members Bcl-2 and Bcl-xL are localized to the mitochondrial membrane, while the pro-apoptotic proteins either locate diffusely in the cytoplasm or associated with the mitochondrial membrane as well. During the induction of apoptosis BH3 only members get activated by transcriptional up regulation (Bax, Puma, Noxa), subcellular relocalization (Bim), dephosphorylation (Bad) or proteolysis (Bid). The activation of the BH3 only members blocks the activity of the anti-apoptotic Bcl-2 proteins in inhibiting pro-apoptotic Bcl-2 members. Additionally, it is assumed that they directly induce the conformational change of Bak and Bax which results in the exposure of the pro-apoptotic BH3 domain and their oligomerization. Subsequently, they insert into the mitochondrial membrane, form pores and elicit cytochrome c release $[54 ; 65 ; 67 ; 68]$.

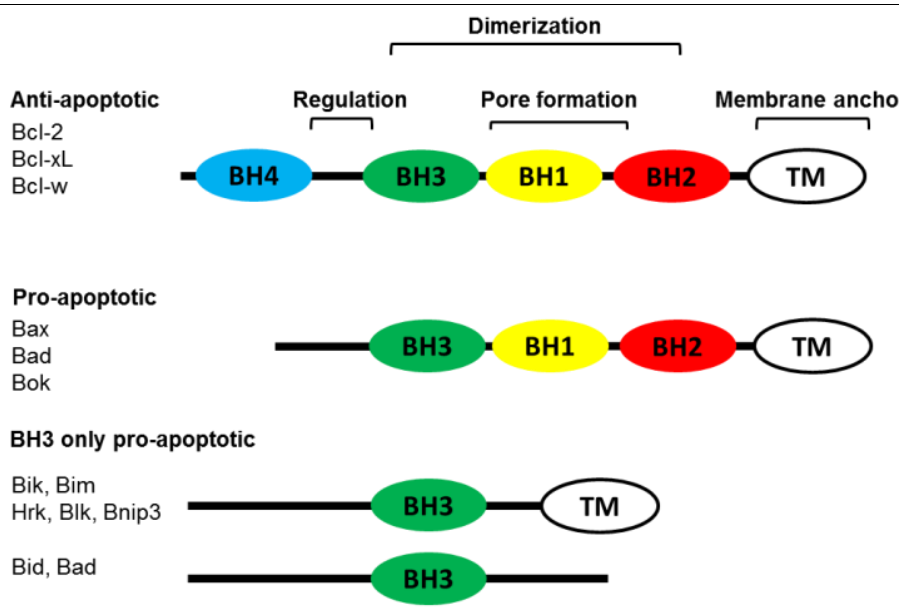

Figure 2.4: Overview of the Bcl-2 family members.

The Bcl-2 family consists of three subfamilies: the anti-apoptotic Bcl-2 proteins that promote cell survival, while the pro-apoptotic proteins and $\mathrm{BH} 3$ only pro-apoptotic proteins evoke apoptosis. Typically, Bcl-2 family members contain up to four conserved Bcl-2 homology $(\mathrm{BH})$ domains designated $\mathrm{BH} 1, \mathrm{BH} 2, \mathrm{BH} 3$ and $\mathrm{BH}$. BH3 domain only members display sequence homology only within the BH3 domain which contains a critical death domain. TM: transmembrane domain. Referring to Zimmermann et al. [67] 


\subsubsection{Bcl-xL}

$\mathrm{Bcl}-\mathrm{xL}$ is an anti-apoptotic protein belonging to the $\mathrm{Bcl}-2$ family. The gene name of $\mathrm{Bcl}-\mathrm{xL}$ is Bcl2L1. Due to the fact that an intron separates the coding region in Bcl2L1 at the same position as Bcl-2, it is suggested that they evolved by duplication and are closely related [69]. Alternative splicing of Bcl2L1 leads to the production of several isoforms. A long version of Bcl2L1, namely Bcl-xL contains all four $\mathrm{BH}$ domains and inhibits programmed cell death. However, an isoform missing 68 amino acids which cover the $\mathrm{BH} 1$ and $\mathrm{BH} 2$ domain is called $\mathrm{Bcl}-\mathrm{xS}$ and antagonizes Bcl-xL. Bcl-xL and Bcl-xS are generated by alternative 5'splice site selection in exon 2 that is regulated by two ceramide-responsive RNA cis elements (CRCEs) [70]. Bcl-xS is highly expressed in cells with high turnover such as developing lymphocytes, whereas Bcl-xL mRNA is found in tissues containing long-lived postmitotic cells such as the adult brain. Bcl-xL expression is more widespread than $\mathrm{Bcl}-\mathrm{xS}$ which is only present in some tissues including the thymus and lymph nodes [70]. Bcl2L1 plays an important role in haematopoiesis as it was shown that Bc12L1 knockout mice die at stage E13 of embryonic development because of cell death in immature neuronal and hematopoietic cells [71].

The half-life of Bcl-xL mRNA is relatively short and the expression Bcl-xL protein is synchronously with the Bcl-xL transcript [72]. So far it is known that Bcl2L1 contains two distinct regions with promoter activity placed upstream of the first coding exon (exon 2) and upstream of the first non-coding exon (exon 1) [73]. Hitherto, it is assumed that both regions are major transcription sites that differ in usage according to cell type and status of differentiation [73]. There are several transcription factors that are involved in the activation of Bcl2L1 including Ets, Rel/NFkB, AP-1 and STAT (Figure 2.5) [72].

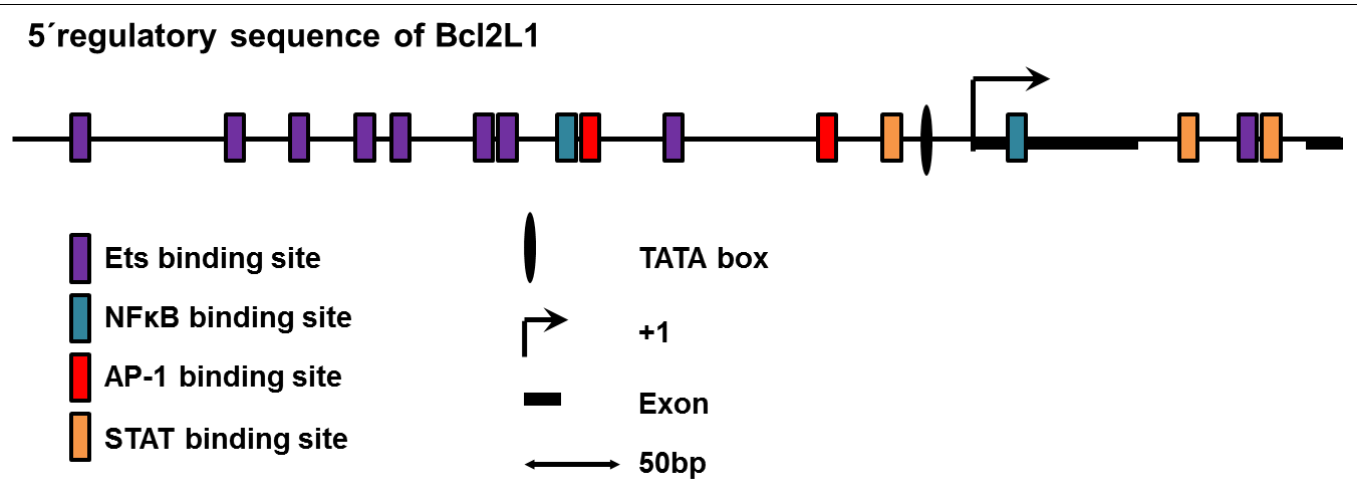

Figure 2.5: The structure of the 5'regulatory sequence of human Bcl2L1.

Shown are the predicted binding sites for the transcription factor complexes Ets, NFkB, STAT and AP-1. The consensus motifs, TATA box, exons and major transcription initiator site $(+1)$ are pictured. Referring to Sevilla et al. [72]. 
The dimeric transcription factor complex AP-1 (activator protein 1) consists of proteins that among others are members of the Jun (e.g. cJun and JunD) and Fos (f.e. cFos) family [74]. Sevilla et al. were able to show that Bcl2L1 was transactivated by c-Fos and c-Jun via the AP1 transcription factor binding site suggesting a critical role for these proteins in regulating Bcl-xL gene expression [75]. The signal transducer and activator of transcription (STAT) proteins are subsequently activated after phosphorylation by JAKs that are downstream effectors of growth factors or cytokines [76]. Activated STATs are found in several tumors and contribute to oncogenesis by affecting Bcl-xL. Thereby it was observed that the constitutive activation of STAT3 correlates with increased Bcl-xL level in squamous cell carcinoma of the head and neck [77]. In addition, STAT5 also seems to be able to induce Bcl-xL gene expression which was shown to be important for leukemic cell lines expressing Bcr-Abl [78]. Additionally, it has been shown that Bcl-xL is up regulated in several cancers including breast, prostate, pancreatic and colorectal cancer which is associated with poor prognosis [76].

Besides the regulation of $\mathrm{Bcl}-\mathrm{xL}$ gene expression, which is mediated by distinct transcription factor binding sites, it was recently shown that Bcl-xL mRNA is also a target for miRNAs. Shimizu et al. have observed that the miRNA let-7 inhibits Bcl-xL gene expression in human hepatocellular carcinoma and that less let-7 miRNA conferred a survival advantage to tumor cells [79]. In this study the miRNA let-7c and miRNA let-7g directly bind to the $3^{\prime}-$ untranslated region of the Bcl-xL mRNA. Therefore an absence of let-7 miRNA is associated with Bcl-xL overexpression [79]. Interestingly, Bcl-xL overexpression seems to be involved in increased metastatic ability as well. It has been demonstrated that transfection of the human breast cancer cell line MDA-MB-435 with Bcl-xL cDNA results in survival advantage accompanied by increased metastatic activity [80]. Concomitant with these findings are results showing that the overexpression of Bcl-xL in MCF-7 human breast cancer cell lines results in the prominent inhibition of apoptosis in response to several chemotherapeutics. Even though these experiments were additionally done with Bcl-2-over-expressing MCF-7 cells, Bcl-xL seems to exhibit a higher potential to inhibit apoptosis [81].

\subsection{Epithelial Mesenchymal Transition}

Epithelial Mesenchymal Transition (EMT) is a process whereby cells change their epithelial morphology into a fibroblast like phenotype (Figure 2.6). EMT is involved in numerous developmental processes including the mesoderm and neural tube formation [82]. However, EMT is also found in association with metastasis [83]. It is assumed, that during carcinogenesis some cells at the periphery of the primary tumor undergo EMT, whereby the cells acquire 
mesenchymal properties. This mesenchymal state facilitates the invasion of cancer cells into local and distant tissues [83]. As a consequence of EMT, cells lose cell specific epithelial marker such as Occludin, Cytokeratin and as a hallmark of EMT, E-Cadherin. E-Cadherin functions as a tumor suppressor through the prevention of invasion and metastasis. During EMT E-Cadherin is repressed and degraded [84]. Instead of epithelial markers cells express mesenchymal markers including Vimentin, Fibronectin and N-Cadherin [85]. Due to the loss of cell polarity, these cells are able to survive without cell-cell contacts, which is important for the metastasis process [83].

Epithelial
phenotype

Figure 2.6: Epithelial Mesenchymal Transition (EMT).

Cells that undergo EMT lose their epithelial phenotype and epithelial markers such as E-Cadherin, Occludin and Cytoceratin. Instead the cells acquire a mesenchymal cell shape and express mesenchymal markers including Vimentin, Fibronectin and N-Cadherin. Referring to Thiery et al. [85].

\subsubsection{Regulation of EMT}

There are several mechanisms which are involved in initiating and executing EMT (Figure 2.7). In cancer cells, the TGF $\beta$ signalling pathway activates multiple EMT-inducing transcription factors, such as Slug, Zeb2 and Goosecoid via Smad activation [82]. Furthermore, the highly conserved canonical Wnt-signalling pathway is involved in initiating EMT [84]. In the inactive state of the Wnt-signalling pathway, $\beta$-Catenin complexes with APC and axin, which results in phosphorylation by GSK3 $\beta$ and degradation of $\beta$-Catenin by the proteasome [84]. But the active Wnt-signalling pathway and the loss of E-Cadherin from the adherens junctions results in the activation of $\beta$-Catenin, which in turn induces the EMT-inducing transcription factors Slug and Twist [82]. Additionally, the multiple tyrosine kinase receptor (TRK) pathways which involve FGFR, EGFR and HGFR activate the gene expression of Slug and Snail through the RAS-MAP kinase pathway [82]. Considering the regulation of E-Cadherin, the transcription factors Snail, Slug and Zeb2 suppress E-Cadherin transcription directly by bind- 
ing to the E-box motifs within the E-Cadherin promoter region. In contrast, Twist, Goosecoid and FOXC2 operate indirectly [82;84].
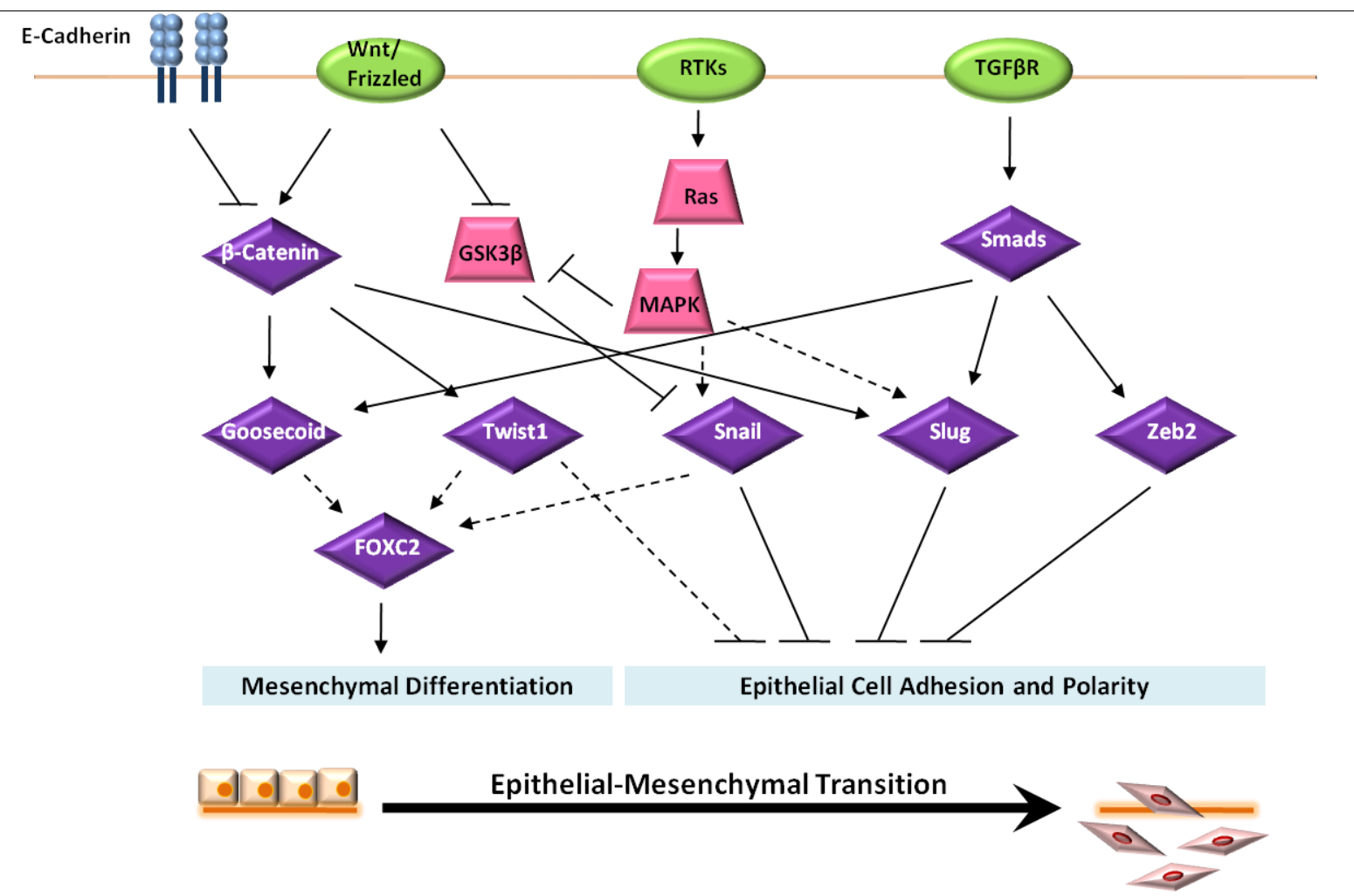

Figure 2.7: Signaling pathways involved in regulation of EMT.

The TGF $\beta$ signalling pathways induce multiple EMT-inducing transcription factors in cancer cells such as Slug, Zeb2 and Goosecoid by the activation of Smads. Activation of the Wnt pathway and loss of E-Cadherin results in the activation of $\beta$-Catenin and subsequently in the activation of distinct EMT-transcription factors including Slug, Twist1 and Goosecoid. Multiple tyrosine kinase receptor (TRK) pathways induce the expression of Snail and Slug via the Ras-MAP kinase pathway. Solid lines indicate direct transcriptional or posttranscriptional regulations. Dashed lines indicate indirect regulation. Referring to Yang et al. [82].

Besides regulation by distinct signalling pathways, it has been shown that EMT is also under the control of micro RNAs (miR). Gregory et al. were able to define five members of the miR-200 family which were specifically down regulated in cells that have undergone EMT, particularly miR-200a, miR-200b, miR-200c, miR-141 and miR-429 [86]. The expression of these miRNAs was diminished in invasive breast cancer cell lines displaying a mesenchymal cell shape. Consistent with these results, the ectopic expression of these miRNAs in mesenchymal cells results in Mesenchymal Epithelial Transition (MET) [86]. The most prominent targets of these miR-200 family members are Zeb1 and Zeb2 [87]. The transcription factors Zeb1 and Zeb2 act thereby as repressors for E-Cadherin, mediated by their binding to the Ebox motif in the proximal E-Cadherin promoter. As mentioned above, both factors are able to induce EMT and are involved in the TGF $\beta$ signalling pathways through binding to Smad coactivators [88]. Interestingly, the miR-200 and Zeb interaction occurs in an inverse manner 
[87]. Correspondingly, the knock down of Zeb1 results in an increase in the expression of all miR-200 family members [89]. Brabletz et al. therefore proposed a double-negative feedback loop between Zeb and miR-200 (Figure 2.8) [87]. Zeb transcription factors are strong EMT inducers, but the overexpression of miR-200 would result in decreased Zeb levels and epithelial differentiation. However, depending on extracellular signals, the direction of this loop can switch and evokes either an epithelial or mesenchymal phenotype [87].
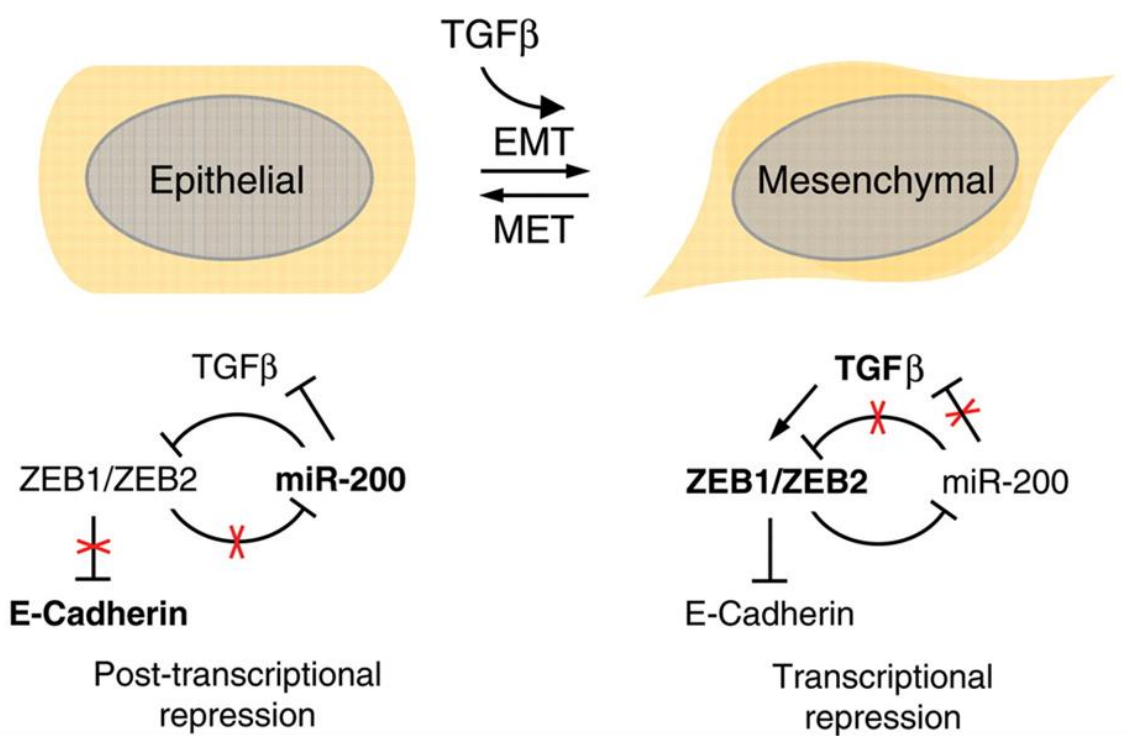

Transcriptional repression

Figure 2.8: Double-negative feedback loop of miR-200 and Zeb during EMT.

miR-200 assisted E-Cadherin gene expression and epithelial morphology by post-transcriptional repression of the transcription factors Zeb1, Zeb2 and of TGF $\beta$. During EMT Zeb1 and Zeb2 are induced by the active TGF $\beta$ signalling pathway, which results in E-Cadherin repression and is associated with a mesenchymal phenotype. This status is mediated by Zeb1/Zeb2 repression of miR-200 transcription in a double-negative feedback loop. This process is reversible with increased expression of miR-200, which induces MET by renewed induction of E-Cadherin gene expression accompanied by an epithelial cell shape. Adopted from Teague et al. [90].

\subsubsection{EMT and cancer}

The molecular links between EMT transcription factors and self-renewal suggest that EMT plays a pivotal role in the early and late stages of the metastasis cascade [91]. Currently, 90\% of tumors originate from epithelial tissue. However, the most apparent indicator differentiating a malignant and metastatic tumor from a benign one is the morphological change from an epithelial morphology to a mesenchymal invasive phenotype [92]. Nevertheless, the occurrence of Epithelial Mesenchymal Transition (EMT) in the progression of epithelial cancers into disseminated metastasis is highly controversial. Actually, epithelial markers, such as cytokeratins, are clinically used to characterize metastatic epithelial tumors in patients [92]. However, importantly not all steps that occur during the developmental process of EMT are 
necessary to establish a metastatic phenotype, meaning that in vivo a partially dedifferentiated cell with a migratory and invasive phenotype should be expected to characterize metastasis [93]. The difficulty with this question is to demonstrate the importance of EMT during metastasis in human cancer progression and the identification of cells that have undergone the EMT in primary human cancer samples. Currently used EMT markers are either expressed in epithelial or in mesenchymal cells, but these markers are not specific enough to identify tumor cells at the EMT stage [82]. Hitherto, it is suggested that EMT in cancer progression depends on the tumor type, and moreover, it is assumed that EMT occurs locally at the invasive front of the primary tumor $[93 ; 94]$. Additionally, it has been recently proposed that EMT-like phenotypes can be classified based on their cell-cell adhesion status. The most prominent examples of tumors that complete EMT in vivo are the carcinosarcomas or metaplastic carcinomas, which represent fewer than $1 \%$ of invasive breast carcinomas, associated with poor prognosis [93]. Based on the expression of cytokeratins or Vimentin, these tumors can be distinguished in epithelial and mesenchymal compartments, respectively [93]. Even luminal A breast cancer, which typically displays an epithelial phenotype, can progress into metastasis including the process of EMT [95]. It is assumed that a subpopulation of cancer cells lose their epithelial properties, while gaining the motile and invasive behavior of mesenchymal cancer cells [95]. More direct evidence for EMT in breast cancer was supplied by Trimboli et al. by developing stromal- and epithelial-specific mouse models that can be used to directly visualize EMT in cancer progression in vivo [96]. Thus, they were able to genetically mark mammary epithelial and stromal cells and follow their fate, while tumors developed in vivo [96].

Furthermore, transcription factors that are involved in the EMT process are transcriptional suppressors, that can partially direct repress epithelial adhesion molecules [91]. Slug, Zeb1 and Twist for example, repress E-Cadherin gene expression, an integral component of adherens junctions. Additionally, the gene expression of the molecule claudin, which is involved in the assembly of tight junctions between adjacent cells is repressed by Snail and Slug [91]. Due to the up-regulation of the mesenchymal marker N-Cadherin, Vimentin and Fibronectin, cells acquire a front-to-back polarization, which in the end results in their ability to migrate and invade surrounding tissues as single cells. Together, the molecular changes of disseminating cancer cells complete the early steps of the metastatic cascade. Hence, EMT presents a possible explanation as to how cancer cells acquire the ability to invade surrounding tissues, survive systemic dissemination and extravasation into distant regions of the organism [91]. The increased levels of several EMT transcription factors seem to be an attribute of several cancers. Twist e.g. is expressed in invasive lobular breast carcinoma, a highly infiltrating tu- 
mor type that exhibits loss of E-Cadherin. Thus, the suppression of Twist in cancer cells is accompanied by an decrease of their metastatic potential in vivo [97].

Invasive breast cancer comprises phenotypically diverse populations of tumor cells, which is reflected in tumor histology, clinical presentation and response to therapy [93]. Al-Hajj et al. observed a minor cell population within human breast cancer samples that contain the ability to form new tumors [98]. Based on their cell surface marker expression, tumorigenic and nontumorigenic cells were characterized as $\mathrm{CD} 24^{-} / \mathrm{CD} 44^{+}$cells (referred as $\mathrm{CD} 44^{+}$) and $\mathrm{CD} 24^{+} / \mathrm{CD} 44^{-}$cells (referred as $\mathrm{CD} 24^{+}$), respectively. Indeed, is has been demonstrated that $\mathrm{CD} 44^{+}$cells from breast cancer patients were more tumorigenic in NOD/SCID mice than $\mathrm{CD} 24^{+}$cells, whereby the resulting xenografts reproduce the heterogeneity of the original tumors [98]. Beyond this, Shipitsin et al. identified that the CD $44^{+}$-cell specific gene expression profile within these heterogeneous breast cancer tissues resembles that of stem cells, normal and $\mathrm{CD} 44^{+}$-tumor cells, more than $\mathrm{CD} 24^{+}$cells from the same tissue [99]. Moreover, they have shown that the TGF $\beta$ signalling pathways, which are also involved in inducing EMT, are specifically active in $\mathrm{CD} 44^{+}$tumor cells, concomitant with a mesenchymal phenotype. Thus, treatment with a TGF $\beta$-inhibitor resulted in dramatic morphological changes in $\mathrm{CD} 44^{+}$tumor cells, accompanied by the acquisition of an epithelial phenotype of these cells within $24 \mathrm{~h}$ [99]. Due to this heterogeneity, it is assumed that a process like EMT contributes to cancer progression, but depends on the tumor type [93].

\subsubsection{EMT involved in chemoresistance}

Tumors that have undergone EMT may acquire resistance to chemotherapy. Correspondingly, epithelial colon carcinoma cell lines that are resistant to Oxaliplatin exhibit mesenchymal properties and express distinct markers for EMT [100]. Moreover, chemoresistance to Paclitaxel in ovarian cancer cells lines was observed to be associated with the expression of EMT markers and loss of epithelial phenotype [101]. Furthermore, highly invasive subpopulations of MCF-7 and MDA-MB-434 cells that have undergone EMT, displayed increased levels of Twist and its target gene Akt2, which was also associated with Paclitaxel resistance [102]. Consistent with these results, it was observed that the reduction of Twist in breast cancer cells results in a partial reverse of the acquired multi drug resistance [85; 103]. Similarly, the suppression of Twist in lung cancer cell line A549 results in sensitivity towards Cisplatin [104].

Moreover, in post-treatment biopsies of patients with breast cancers that were medicated with Anthracycline-Taxane chemotherapy, an increase of $\mathrm{CD} 44^{+} / \mathrm{CD} 24^{-}$cells was observed which 
highly expressed EMT-associated genes [4]. So called stem cell like cancer cells display a $\mathrm{CD} 44^{+} / \mathrm{CD} 24^{-}$surface profile as well and can be regulated by EMT transcription factors [91]. It has been shown that these stem cell like cancer cells express increased level of ABCtransporter and multi-drug-resistant proteins, protecting them from chemotherapeutic treatment [105]. Additionally, it is often observed that $\mathrm{CD}^{4} 4^{+} / \mathrm{CD} 24^{-}$cells contain more active aldehyde dehydrogenase 1 (ALDH 1) which constitutes a detoxifying enzyme that oxidizes intracellular aldehydes and induces resistance to alkylating agents $[106 ; 107 ; 108]$. Finally, the inhibition of apoptosis is a crucial mechanism to escape chemotherapy. Thereby, Twist has been shown to inhibit apoptosis by several mechanisms. These mechanisms include the inhibition of the pro-apoptotic properties of the Myc oncogene, the activation of the NFkB signalling pathway and through the repression of p53-induced pro-apoptotic genes [91].

\subsubsection{Model system for EMT}

Weinberg and colleagues (MIT Boston, USA) have established a model system for EMT containing the Human Mammary Large T-antigen immortalized Epithelial cells, named HMLE (Figure 2.9) [109]. To create this model system, primary Human Mammary Epithelial Cells (HMEC) from reduction mammoplasty tissue samples from a healthy female donor were immortalized using the SV-40 large T antigen of the simian virus 40 and the subunit of the human telomerase hTERT. Thereby, hTERT maintains the telomeres during the cell cycle, while the SV-40 large T antigen is involved in the inactivation of the p53 and pRB pathways [109]. Upon this immortalization it is possible to culture these cells. However, to work with a model system that is more cancer-orientated and is indeed able to induce tumors, these HMLEs were additionally transformed with the oncogene haRAS and thus acquire a constitutive mitogenic signal [109]. The EMT model system predominantly used in this thesis is HMLE RAS: $\mathrm{Hu}-$ man Mammary Large T-Antigen immortalized Epithelial HaRas expressing cells.

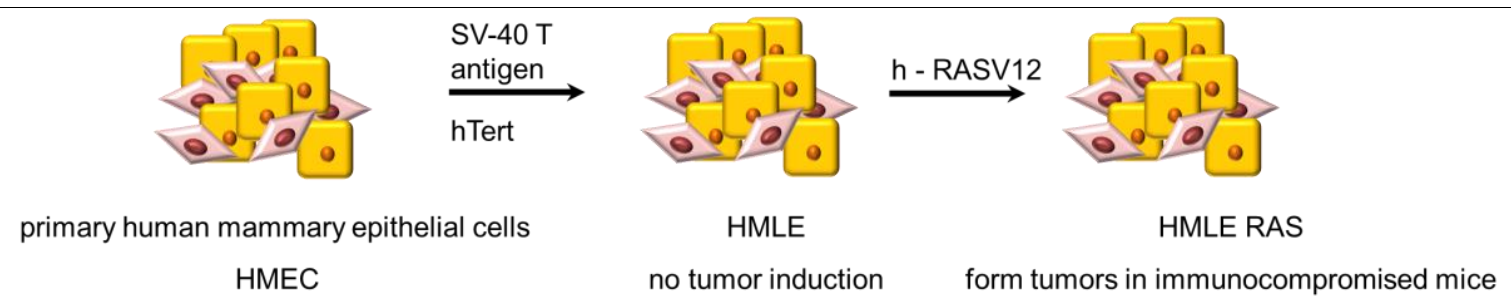

Figure 2.9: Stepwise transformation of HMECs to create the HMLE RAS model system.

Initially, HMECs were immortalized with the SV40 large T antigen and the telomerase subunit hTERT to obtain the HMLEs. Further, HMLE cells were transformed with the haRAS oncogene to receive tumorigen HMLE RAS cells. 
Every model system consists of two different cell lines (Figure 2.10). On the one hand are the $24^{+}$HMLE and $24^{+}$RAS cells, respectively, which display an epithelial phenotype and are typically positive for the surface marker CD24. The $24^{+} \mathrm{HMLE}$ and $24^{+} \mathrm{RAS}$ cells are not able to induce tumors, which is a characteristic of cells before EMT. However, it is possible to isolate a subset of cells from the $24^{+}$HMLE and $24^{+}$RAS cells termed Mesenchymal subpopulation (MSP) that express mesenchymal markers such as Vimentin and N-Cadherin and are accompanied by a mesenchymal morphology. MSP and MSP RAS cells, respectively, are positive for CD44, able to migrate and are typical of cells that have undergone the process of EMT. Additionally, Weinberg and colleagues have shown that MSP RAS in contrast to $24^{+}$RAS are highly invasive and tumorigenic [110].

Scheel et al. have shown that MSP cells remain stable in the mesenchymal state and that the induction of EMT and subsequent maintenance of the mesenchymal state occurs spontaneously and is not generated by an experimental procedure [110]. Additionally, they observed that MSP cells express less Wnt-repressors such as DKK1 and sFRP than their parental counterpart, the $24^{+} \mathrm{HMLE}$ cells. Consistent with this, the MSP cells express the non-canonical Wnt ligands Wnt5a and Wnt16. Moreover, Scheel et al. found activation of the canonical Wnt pathway in MSP cells as well. At this they were able to determine active TOPFlash Luciferase reporter assays, indicating that this pivotal EMT signalling pathway is highly induced in MSP cells [110].
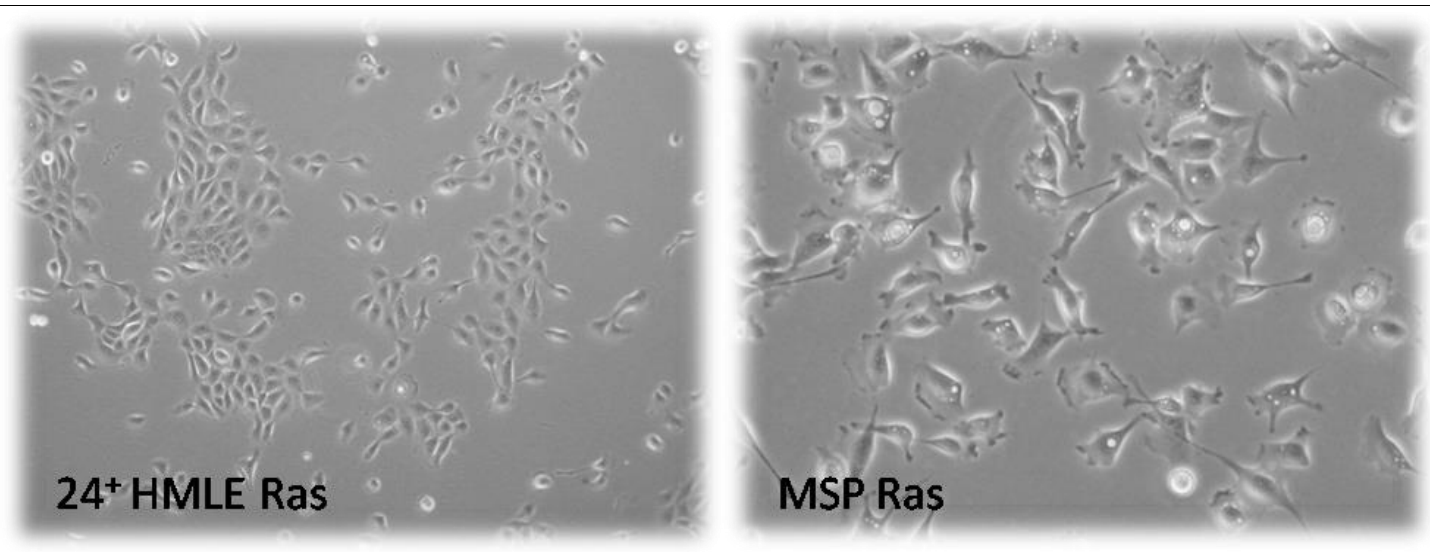

Figure 2.10: HMLE RAS cells as model system for EMT.

$24^{+}$HMLE Ras display an epithelial morphology, are highly positive for the surface marker CD24, lack efficient migration and express E-Cadherin. $24^{+}$HMLE Ras show characteristics of cells before EMT. MSP Ras exhibit a mesenchymal phenotype and express the mesenchymal markers Vimentin and N-Cadherin. MSP Ras are highly invasive and express the surface marker CD44 but not CD24. MSP Ras cells show characteristics of cells that have undergone EMT and contain a highly active Wnt-signalling pathway.

Mani et al. have shown, that EMT induced by the overexpression of the transcription factor Twist in immortalized human mammary epithelial cells (HMLE), not only results in the ac- 
quisition of a mesenchymal phenotype, but rather results in the expression of stem cell markers [111]. Using flow cytometry, they were able to detect CD24 $/ \mathrm{CD} 44^{+}$cells within the mesenchymal population, which displayed the ability to form mammospheres, an attribute that is concomitant with stem cells. Indeed, HMLEs that underwent EMT by ectopic expression of Twist formed 30-fold more mammospheres than HMLEs infected with the control vector [111]. These findings by Mani et al. strongly imply that the MSP and MSP RAS cells used in our EMT system obtain these stem cell markers as well.

\subsection{Objective}

As described in detail above, a major problem in treating cancer is the acquisition of chemoresistance by cancer cells. It was recently published, that tumor cells that survive chemotherapy differ from the major tumor population and contain tumor initiating properties that are associated with resistance to chemotherapy [4]. New knowledge of this subpopulation revealed disseminating cells that change their behaviour and morphology during the process of EMT. These cells acquire the ability to invade local and distant tissues, form metastasis and escape chemotherapeutic treatment. Considering the various ways in which tumor cells can manipulate their chemoresponse, the question arises as to how these cells acquire chemoresistance and how EMT comes into play. What differs in cells upon EMT in comparison to their parental cells?

To investigate how EMT affects the response to chemotherapy, we were provided with a model system for EMT containing the Human Mammary Large T-Antigen immortalized Epithelial HaRas expressing cells (HMLE RAS) by Weinberg and colleagues (MIT Boston, USA). This model system is composed of two different cell lines. On the one hand are the parental $24^{+}$RAS cells that display an epithelial morphology and lack efficient migration. On the other hand are the MSP RAS cells (mesenchymal subpopulation) that originate from the $24^{+}$RAS cells. MSP RAS cells contain activated EMT signalling pathways accompanied by a mesenchymal cell shape and high invasivity. Preliminary experiments have confirmed that these cells are resistant to several chemotherapeutics and encouraged us to determine how these cells became chemoresistant during the process of EMT and which mechanism is behind it. We wanted to figure out the level at which the resistance mechanism occurs and moreover, we were interested in overcoming resistance in cells upon EMT. The correlation of EMT, tumor-initiating cells and chemotherapeutic resistance is a recent field in carcinogenesis. Understanding the mechanisms that are involved in chemoresistance regarding EMT is most important to develop novel therapeutic approaches to medicate cancer. 


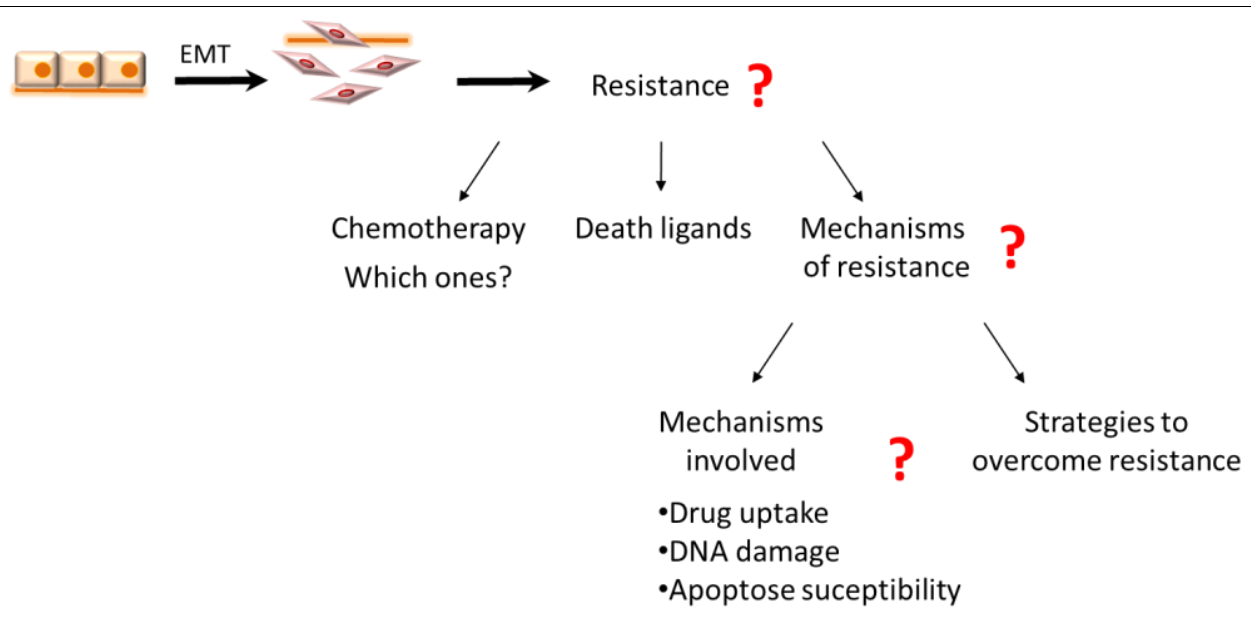

Figure 2.11: Objective at a glance. 


\section{Material}

\subsection{Technical devices}

Table 3.1: Technical devices

Device

Blotting chamber

Cell counting chamber Neubauer improved

Centrifuge 5415R

Centrifuge $5810 R$

Centrifuge Megafuge 1.OR

Chemiluminescence imager Chemocam HR 163200

Cytometer Celigo

DNA gel chamber

Electrophoresis system, for SDS-PAGE

FACS machine Guava PCA-96 Base System Foil swelding machine Vacupack plus

Freezer $-20^{\circ} \mathrm{C}$

Freezer $-80^{\circ} \mathrm{C}$

Heating Block

Heating Block $H L C$

Ice-machine $B 100$

Incubator for bacteria

Incubator for bacteria Minitron

Laminar flow cabinet Hera Safe

Liquid nitrogen tank LS 4800

Magnetic stirrer MR Hei-Standard

Magnetic stirrer MR3001

Microscope Axovert 40C

Microscope, Axioscope 2 Plus

Microscope, confocal Zeiss Confocal LSM

510 meta

Microwave

Mini Centrifuge $M C F-2360$

PCR machine for qRT-PCR Chromo4

PCR machine Thermocycler T personal pH-meter WTW-720

\section{Company}

Biozym, Hessisch Oldendorf, Germany

Brand, Wertheim, Germany

Eppendorf, Hamburg, Germany

Eppendorf

Heraeus, Thermo Scientific, Waltham, MA, United States

Intas Science Imaging Instruments, Göttingen, Germany

Cyntellect, San Diego, CA, United States

Biotech Service Blu, Schauenburg, Germany

Amersham Biosciences, GE Healthcare, Lit-

tle Chalfont, United Kingdom

Millipore, Merck, Darmstadt, Germany

Krups, Groupe SEB, Lyon, France

Liebherr, Bulle, Switzerland

Heraeus, Thermo Scientific

Grant Instruments, Hillsborough, NJ, United

States

HLC Biotech, Ditabis, Pforzheim, Germany

Ziegra, Isernhagen, Germany

Memmert, Schwabach, Germany

Infors HT, Basel, Switzerland

Heraeus, Thermo Scientific

Taylor-Wharton, Theodore, AL, United

States

Heidolph, Schwabach, Germany

Heidolph

Zeiss, Oberkochen, Germany

Zeiss

Zeiss

Cinex, Lippstadt, Germany

LMS, Tokyo, Japan

Bio-Rad Laboratories

Biometra, Göttingen, Germany

WTW, Weilheim, Germany 
Pipet Multipette

Pipet, electric Portable-XP

Pipets Eppendorf Research Series 2100

(0.1-2.5 $\mu \mathrm{l} ; 0.5-10 \mu \mathrm{l} ; 10-100 \mu \mathrm{l} ; 100-1000 \mu \mathrm{l})$

Pipette, multichannel Research Plus

Power supply unit Powerpack P25T

Refrigerator $4^{\circ} \mathrm{C}$

Roller RM5 V-30

Rotator PTR 300

Scales Acculab ALC-6100.1

Scales LE623S

Scanner CanoScan $8600 F$

Sequencer, automated ABI 3100

Shaker PROMAX 2020

Sonication device Bioruptor

Spectrophotometer NanoDrop ND-1000

Thermomixer comfort

Vacuum pump

Vortex Genie 2

Water bath $T W 20$
Eppendorf

Drummond, Broomal, PA, United States

Eppendorf

Eppendorf

Biometra

Liebherr

CAT, Staufen, Germany

Grant Instruments

Sartorius, Göttingen, Germany

Sartorius

Canon, Tokyo, Japan

Applied Biosystems, Life Technologies

Heidolph

Diagenode, Liège, Belgium

PeqLab, Erlangen, Germany

Eppendorf

IBS Integra Biosciences, Fernwald, Germany Scientific Industries, Bohemia, NY, United States

Julabo Labortechnik, Seelbach, Germany

\subsection{Consumables}

Table 3.2: Consumables

\section{Product}

12-well plates for microscopy

96-well plates for qPCR

Cell culture flasks $\left(25 \mathrm{~cm}^{2}, 75 \mathrm{~cm}^{2}, 125 \mathrm{~cm}^{2}\right)$

Cell culture plates (6-well, 12-well)

Cell scraper $(16 \mathrm{~cm}, 25 \mathrm{~cm})$

Cryo tubes Cryoline

Filter tips $(10 \mu 1)$

Filter tips $(20 \mu 1,200 \mu 1,1,000 \mu 1)$

Parafilm

Pipet tips $(10 \mu 1,20-200 \mu 1,1,000 \mu 1)$

Protran nitrocellulose transfer membrane

Reaction tube $(0.2 \mathrm{ml})$

Reaction tube $(0.5 \mathrm{ml}, 1.5 \mathrm{ml}, 2.0 \mathrm{ml})$

Reaction tube $(15 \mathrm{ml}, 50 \mathrm{ml})$

Safe-lock reaction tube $(1.5 \mathrm{ml})$

Sterile filter

Syringe

\section{Company}

Corning, Corning, NY, United States

4titude, Wotton, United Kingdom

Greiner, Frickenhausen, Germany

Greiner

Sarstedt

Nunc, Thermo Scientific

Starlab, Hamburg, Germany

Sarstedt

Brand

Greiner

Whatman, Dassel, Germany

Sarstedt

Eppendorf

Greiner

Eppendorf

Millipore, Merck

Henke-Sass, Wolf, Tuttlingen, Germany 
Syringe canula (different sizes)

Transparent sealing foil for 96-well plate

Whatman paper
B.Braun, Melsungen, Germany

4titude

Whatman

\subsection{Chemicals and reagents}

Table 3.3: Chemicals and reagents

Substance

Acetic acid

Acrylamide-bisacrylamide

Albumin Fraction V (Bovine Serum Albumine,

BSA)

Ammonium persulfate (APS)

Ammonium sulfate $\left(\left(\mathrm{NH}_{4}\right)_{2} \mathrm{SO}_{4}\right)$

Aprotinin

Bromophenol blue

Calcium chloride dihydrate $\left(\mathrm{CaCl}_{2} \times 2 \mathrm{H}_{2} \mathrm{O}\right)$

Chloroform

Crystal violet

Dimethyl sulfoxide (DMSO)

Dithiotreitol (DTT)

DNA ladder

Deoxynucleotide triphosphates (dNTPs)

Ethanol 99.8\%

Ethanol $99.9 \%$ p.a. (EtOH)

Ethylene diamine tetraacetatic acid (EDTA)

Ethylene glycol tetraacetic acid (EGTA)

FCS

Glycerol

Glycine

Glycogen

Glycogen blue

Guava ICF Cleaning Solution

Guava Viacount reagent

HEPES

Hydrogen chloride $(\mathrm{HCl})$

Immersion oil

Isoamyl alcohol

Isofluran

Isopropanol

Leupeptin Hemisulfat

Lipofectamine 2000

Magnesium chloride $\left(\mathrm{MgCl}_{2}\right)$ for PCR

Magnesium chloride hexahydrate $\left(\mathrm{MgCl}_{2} \mathrm{X}\right.$

\section{Company}

Roth, Karlsruhe, Germany

Roth

Roth

Roth

Roth

Applichem

Sigma-Aldrich

Roth

Roth

Sigma-Aldrich

AppliChem

Sigma-Aldrich

Fermentas, Thermo Scientific

Bio-Budget, Krefeld, Germany

Roth

Merck

Roth

Roth

Thermo Scientific HyClone

Roth

Roth

Fermentas, Thermo Scientific

Ambion, Life Technologies

Millipore, Merck

Millipore, Merck

Roth

Roth

Zeiss

Roth

Baxter

Th. Geyer, Renningen, Germany

Applichem

Invitrogen, Life Technologies

Fermentas, Thermo Scientific

Roth 
$\left.6 \mathrm{H}_{2} \mathrm{O}\right)$

Matrigel $^{\mathrm{TM}}$ Basement Membrane Matrix

Methanol >99\% (MetOH)

Microcystin-LR

Milk powder

Nonidet P-40 substitute (NP-40)

Nuclease free water

Pefabloc SC

Pepstatin A

Ponceau S

Potassium chloride $(\mathrm{KCl})$

Potassium glutamate (KGlu)

Potassium hydrogenphosphate $\left(\mathrm{KH}_{2} \mathrm{PO}_{4}\right)$

Prestained Protein Ladder

RNase inhibitor

Rotiphorese Gel 30

Sodium acetate $(\mathrm{NaAc})$

Sodium bicarbonate $\left(\mathrm{NaHCO}_{3}\right)$

Sodium chloride $(\mathrm{NaCl})$

Sodium deoxycholate

Sodium dodecyl sulfate (SDS)

Sodium hydrogenphosphate heptahydrate $\left(\mathrm{Na}_{2} \mathrm{HPO}_{4} \times 7 \mathrm{H}_{2} \mathrm{O}\right)$

Sodium hydroxide $(\mathrm{NaOH})$

Sodium orthovanadate

SYBR green

Tetracycline

Tetramethylethylenediamine (TEMED)

Trasylol

Trehalose

Trisamine (Tris)

Triton X-100

Trizol

Tween 20

$\beta$-Mercaptoethanol
BD Biosciences

Roth

JenaBioscience

Roth

Sigma Aldrich

Ambion, Life Technologies

Roth

Applichem

Roth

Roth

Roth

Roth

Fermentas, Thermo Scientific

Fermentas, Thermo Scientific

Roth

Roth

Roth

Roth

Applichem

Roth

Roth

Sigma-Aldrich

Sigma-Aldrich

Invitrogen, Life Technologies

Sigma-Aldrich

Roth

Bayer, Leverkusen, Germany

Sigma-Aldrich

Roth

Applichem

Invitrogen, Life Technologies

Applichem

Roth

\subsubsection{Buffers and solutions}

Table 3.4: Buffers and solutions 
Cell lysis buffer

Laemmli buffer, 6x

PBS, $\mathrm{pH} 7.4$

TBS/T, pH 7.5

qRT-PCR reaction buffer, 10x

qRT-PCR reaction mix, 25x

Ripa lysis buffer, pH 7.5
$100 \%$ Ripa lysis buffer

2M Urea

$1 \mu \mathrm{g} / \mathrm{ml}$ Aprotinin

$1 \mu \mathrm{g} / \mathrm{ml}$ Leupeptin Hemisulfat

$1 \mu \mathrm{g} / \mathrm{ml}$ Pepstatin A

$1 \mathrm{mM}$ Pefabloc SC

$0.5 \mu \mathrm{g} / \mathrm{ml}$ Microcystin

$0.35 \mathrm{M}$ Tris, $\mathrm{pH} 6.8$

$30 \%$ Glycerin

$10 \%$ SDS

$9.3 \%$ DTT

$0.02 \%$ Bromphenol blue

dissolved in $\mathrm{H}_{2} \mathrm{O}$

$24 \mathrm{mM} \mathrm{NaCl}$

$0.27 \mathrm{mM} \mathrm{KCl}$

$0.81 \mathrm{mM} \mathrm{Na}_{2} \mathrm{HPO}_{4} \times 7 \mathrm{H}_{2} \mathrm{O}$

$0.15 \mathrm{mM} \mathrm{KH}_{2} \mathrm{PO}_{4}$

dissolved in $\mathrm{H}_{2} \mathrm{O}$

$50 \mathrm{mM}$ Tris

$150 \mathrm{mM} \mathrm{NaCl}$

$0.1 \%$ Tween 20

dissolved in $\mathrm{H}_{2} \mathrm{O}$

750mM Tris-HCl, $\mathrm{pH} 8.5$

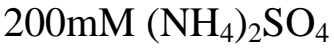

$0.1 \%$ Tween 20

dissolved in $\mathrm{H}_{2} \mathrm{O}$

1x qRT-PCR reaction buffer 10x

$3 \mathrm{mM} \mathrm{MgCl} 2$

1:80.000 SYBR Green

$0.2 \mathrm{mM}$ dNTPs

20U/ml Taq polymerase

$0.25 \%$ Triton X-100

$300 \mathrm{mM}$ Trehalose in $10 \mathrm{mM}$ Tris, $\mathrm{pH} 8.5$

dissolved in water

$1 \%$ Triton $\mathrm{X}-100$

$1 \%$ Na deoxycholat

$0.1 \%$ SDS

$150 \mathrm{mM} \mathrm{NaCl}$

$10 \mathrm{mM}$ EDTA 
20mM Tris, $\mathrm{pH} 7.5$

50.000KIU Trasylol

dissolved in $\mathrm{H}_{2} \mathrm{O}$

SDS running buffer

$25 \mathrm{mM}$ Tris

86.1mM Glycin

$3.5 \mathrm{mM}$ SDS

dissolved in $\mathrm{H}_{2} \mathrm{O}$

Western blot blocking solution

5\% milk powder

dissolved in TBS/T

Western salts, $\mathrm{pH} 8.3$

$0.25 \mathrm{M}$ Tris, $\mathrm{pH} 8.3$

$0.19 \mathrm{M}$ Glycin

$0.02 \%$ SDS

dissolved in $\mathrm{H}_{2} \mathrm{O}$

Western blot transfer buffer

1x Western salts 10x

$20 \% \mathrm{MeOH}$

dissolved in $\mathrm{H}_{2} \mathrm{O}$

\subsubsection{Chemotherapeutics and pharmological inhibitors}

Table 3.5: Chemotherapeutics

\begin{tabular}{lll} 
Chemotherapeutic & Commercial name & Company \\
\hline Carboplatin & Carbomedac ${ }^{\circledR}$ & medac Gesellschaft für klinische \\
& & Spezialpräparate mbH \\
Cisplatin & Cisplatin KL & Neocorp AG, Weilheim \\
Doxorubicin & - & Santa Cruz \\
Neocarcinostatin & - & Sigma Aldrich
\end{tabular}

Table 3.6: Pharmacological inhibitors

\begin{tabular}{llll} 
Name & $\begin{array}{l}\text { Commercial } \\
\text { name }\end{array}$ & Target & Company \\
\hline Akt inhibitor & LY294002 & Akt & Cell Signaling \\
Bcl-xL inhibitor & Gossypol & Bcl-xL & Selleckchem \\
CHX & Cycloheximide & A-site of ribosomes & Sigma Aldrich \\
HSP90 inhibitor & 17AAG & HSP90 & NCI \\
HSP90 inhibitor & 17DMAG & HSP90 & NCI \\
JNK inhibitor & SB600125 & JNK & Sigma Aldrich \\
Mek1 inhibitor & U0126 & Mek1 & Cell Signaling \\
MG132 & Z-Leu-Leu-Leu-al & proteasome & Sigma Aldrich \\
mTOR inhibitor & Rapamycin & mTOR & Calbiochem \\
p38 inhibitor & SB203580 & p38 & Biomol \\
TGF $\beta$ inhibitor & SB431542 & TGF $\beta$ & Sigma Aldrich
\end{tabular}




\subsubsection{Enzymes and proteins}

Table 3.7: Enzymes and proteins

Reagent

M-MuLV Reverse transcriptase (RT)

Rnase inhibitor

Taq DNA polymerase (Taq)

$\mathrm{TNF} \alpha$ (in sterile $\mathrm{H}_{2} \mathrm{O}$ )

Trail (in sterile $\mathrm{H}_{2} \mathrm{O}$ )

\section{Company}

New England Biolabs

New England Biolabs

Primetech LTD, Minsk, Belarus

Gibco

Gibco

\subsection{Kits}

Table 3.8: Kits

Name

\section{Company}

BCA Protein Assay Kit

Guava Check Kit

Guava® Multi Caspase FAM Kit

Immobilon Western HRP Substrate Peroxide

Solution

NucleoBond ${ }^{\circledR}$ AXG

SuperSignal West Femto Maximum Sensitivity

Substrate

TaqMan® MicroRNA Assay
Thermo Scientific

Millipore, Merck

Millipore, Merck

Millipore, Merck

Mecherey Nagel

Thermo Scientific

Invitrogen

\subsection{Oligonucleotides}

Table 3.9: Small interfering RNAs

\begin{tabular}{|c|c|c|}
\hline Target & siRNA ID & Sequence \\
\hline Negative control No.2 & - & undisclosed \\
\hline \multirow[t]{2}{*}{ Bcl-xL \#1 } & s1920 & sense: 5’-AUACUUUUGUGGAACUCUAtt-3' \\
\hline & & antisense: 5'-UAGAGUUCCACAAAAGUAUcc-3' \\
\hline \multirow[t]{2}{*}{ Bcl-xL \#2 } & s1921 & sense: 5’-GCUGGAGUCAGUUUAGUGAtt-3’' \\
\hline & & antisense: 5'-UCACUAAACUGACUCCAGCtg-3' \\
\hline \multirow[t]{2}{*}{ Bcl-xL \#3 } & s1922 & sense: 5’-GGAACUCUAUGGGAACAAUtt-3’' \\
\hline & & antisense: 5'-AUUGUUCCCAUAGAGUUCCac-3' \\
\hline \multirow[t]{2}{*}{ cFos \#1 } & s5339 & sense: 5’-CACUCCAAGCGGAGACAGAtt-3 \\
\hline & & antisense: 5'-UCUGUCUCCGCUUGGAGUGta-3' \\
\hline \multirow[t]{2}{*}{ cFos \#2 } & s5340 & sense: 5’-CUUCCUUCGUCUUCACCUAtt-3’' \\
\hline & & antisense: 5'-UAGGUGAAGACGAAGGAAGac-3' \\
\hline \multirow[t]{2}{*}{ cJun \#1 } & s7658 & sense: 5’-GGCACAGCUUAAACAGAAAtt-3’' \\
\hline & & antisense: 5'-UUUCUGUUUAAGCUGUGCCac-3' \\
\hline \multirow[t]{2}{*}{ cJun \#2 } & s7659 & sense: 5’-CCAAGUGCCGAAAAAGGAAtt-3’’ \\
\hline & & antisense: 5'-UUCCUUUUUCGGCAUUGGag-3' \\
\hline cJun \#3 & s7660 & sense: 5’-GGAUCAAGGCGGGAGAGGAAtt-3’' \\
\hline
\end{tabular}




\begin{tabular}{|c|c|c|}
\hline & & antisense: 5'-UUCCUCUCCGCCUUGAUCCgc-3' \\
\hline $\mathrm{HSP} 90 \alpha$ & s6994 & $\begin{array}{l}\text { sense: 5'-CUAUGGGUCGGUGGAACAAAtt-3' } \\
\text { antisense: 5'-UUUGUUCCACGACCCAUAGgt-3 }\end{array}$ \\
\hline HSP90 $\beta$ & s6999 & $\begin{array}{l}\text { sense: 5'-GGAUGACAGCGGUAAGGAUtt-3' } \\
\text { antisense: 5'-AUCCUUACCGCUGUCAUCCtc-3' }\end{array}$ \\
\hline JunD \#1 & s7664 & $\begin{array}{l}\text { sense: 5'-GUUUCUACUCGGGAACAAAtt-3' } \\
\text { antisense: 5'-UUUGUUCCCGAGUAGAAACat-3' }\end{array}$ \\
\hline JunD \#2 & s7665 & $\begin{array}{l}\text { sense: 5'-UCCACAUUCCUGUUUGUAAtt-3' } \\
\text { antisense: 5'-UUACAAACAGGAAUGUGGAct-3' }\end{array}$ \\
\hline Dicer \#1 & s23754 & $\begin{array}{l}\text { sense: 5'-GAUCCUAUGUUCAAUCUAAtt-3' } \\
\text { antisense: 5'-UUAGAUUGAACAUAGGAUCga-3' }\end{array}$ \\
\hline Dicer \#2 & s23755 & $\begin{array}{l}\text { sense: 5'-CAGCAUACUUUAUCGCCUUtt-3' } \\
\text { antisense: 5'-AAGGCGAUAAAGUAUGCUGgg-3' }\end{array}$ \\
\hline
\end{tabular}

All siRNA are silencer select from Ambion ${ }^{\circledR}$ Life Technologies.

Table 3.10: Primer

\begin{tabular}{|c|c|}
\hline Name & Sequence \\
\hline \multirow[t]{2}{*}{ Axin2 } & for 5'-TCCCCACCTTGAATGAAGAA-3' \\
\hline & rev 5'-TGGTGGCTGGTGCAAAGA-3' \\
\hline \multirow[t]{2}{*}{$\mathrm{Bcl} 2 \mathrm{~L} 1$} & for 5'-ACCGGCGGGCATTCAGTGAC-3' \\
\hline & rev 5'-TGAGCCCAGCAGAACCACGC-3 \\
\hline \multirow[t]{2}{*}{ Bcl-xL } & for 5'-CCTTTTTCTCCTTCGGCGGGGC-3' \\
\hline & rev 5’-GCCCTTTCGGCTCTCGGCTG-3’' \\
\hline \multirow[t]{2}{*}{ Bcl-xS } & for 5'AGAGCTTTGAACAGGATACTTTTGTGGA-3 \\
\hline & rev 5'-TGAGCCCAGCAGAACCACGC-3 \\
\hline \multirow[t]{2}{*}{ CD24 } & for 5'-ACCCAGCATCCTGCTAGACGCG-3' \\
\hline & rev 5'-AGTTGGATTTGGGGCCAACCCAGA-3' \\
\hline \multirow[t]{2}{*}{ cFos \#1 } & for 5'-CTTACTACCACTCACCCGCAG-3'́ \\
\hline & rev 5'-GTGGGAATGAAGTTGGCACTG-3' \\
\hline \multirow[t]{2}{*}{ cFos \#2 } & for 5'-CTACCACTCACCCGCAGAC-3 \\
\hline & rev 5'-GTCCGGACTGGTCGAGAT-3' \\
\hline \multirow[t]{2}{*}{ cJun } & for 5'-CCCCAAGATCAACAGA-3' \\
\hline & rev 5'-CCGTTGCTGGGATTAT-3' \\
\hline \multirow[t]{2}{*}{ c-Myc } & for 5'-AGCCAGCGGTCCGCAACCCTTGCC-3' \\
\hline & rev 5'-AGCTCGAATTTCTTCCAGATATCC-3’' \\
\hline \multirow[t]{2}{*}{ E-Cadherin } & for 5’-CCTGGACGCTCGGCCTGAAG-3’' \\
\hline & rev 5'-ATAAGGCGGGGCTGTGGGGT-3' \\
\hline \multirow[t]{2}{*}{ HSP90 pan } & for 5'-CCCTTCCACGTCTCTGCATT-3’' \\
\hline & rev 5'-CТАААССТССТССТССТССА3’' \\
\hline \multirow[t]{2}{*}{ JunD \#1 } & for 5'-CCCCTTCGGTTCTTTCGACC-3' \\
\hline & rev 5'-CGGGCGAACCAAGGATTACA-3' \\
\hline JunD \#2 & for 5'-CCCTCAAGAGTCAGAACACGG-3 \\
\hline
\end{tabular}


MMP7

Twist

Vimentin

Zeb1

Zeb2

36B4 rev 5'-GACGTGGCTGAGGACTTTCT-3'

for 5'-GAGGAGCTCATGGGGACTC-3'

rev 5'-CCATAGGTTGGATACATCACTGC-3'

for 5'-GGAGTCCGCAGTCTTACG-3'

rev 5'-TCTGGAGGACCTGGTAGAGG-3'

for 5'-CGTGTATGCCACGCGCTCCT-3'

rev 5'-TCGAGCTCGGCCAGCAGGAT-3'

for 5'-GCGCAGAAAGCAGGCGAACCC-3'

rev 5'-CCCTTCCTTTCCTGTGTCATCCTCC-3'

for 5'-AACGGTCCTGCCTCCCGACA-3'

rev 5'-AGTTCGCATGGACTCGGCGC-3'

for 5'-GATTGGCTACCCAACTGTTG-3'

rev 5'-CAGGGGCAGCAGCCACAAA-3'

All primers are from Metabion.

\subsection{Antibodies}

Table 3.11: Primary Antibodies

\begin{tabular}{|c|c|c|c|c|}
\hline Name & MW [kDa] & Source & Dilution & Company (Cat.No.) \\
\hline$\beta$-Actin & 42 & $\begin{array}{l}\text { mouse } \\
\text { monoclonal }\end{array}$ & $1: 100,000$ & Santa Cruz (sc-24) \\
\hline Akt & 60 & $\begin{array}{l}\text { rabbit } \\
\text { polyclonal }\end{array}$ & $1: 1000$ & Cell Signaling (9272) \\
\hline Bcl-2 & 26 & $\begin{array}{l}\text { rabbit } \\
\text { polyclonal }\end{array}$ & $1: 1000$ & Cell Signaling (2870) \\
\hline Bcl-xL & 30 & $\begin{array}{l}\text { rabbit } \\
\text { polyclonal }\end{array}$ & $1: 2000$ & Cell Signaling (2764) \\
\hline Bcl-xL & 30 & $\begin{array}{l}\text { mouse } \\
\text { monoclonal }\end{array}$ & $1: 1000$ & Santa Cruz (sc-8392) \\
\hline Caspase 3 (cleaved) & $17 / 19$ & $\begin{array}{l}\text { rabbit } \\
\text { polyclonal }\end{array}$ & $1: 1000$ & Cell Signaling (9664) \\
\hline Caspase 3 (full) & 35 & $\begin{array}{l}\text { rabbit } \\
\text { polyclonal }\end{array}$ & $1: 1000$ & Cell Signaling (9665) \\
\hline cJun & 39 & $\begin{array}{l}\text { rabbit } \\
\text { polyclonal }\end{array}$ & $1: 1000$ & Abcam (ab32137) \\
\hline
\end{tabular}




\begin{tabular}{|c|c|c|c|c|}
\hline Hifl $\alpha$ & 120 & $\begin{array}{l}\text { rabbit } \\
\text { polyclonal }\end{array}$ & $1: 1000$ & Cell Signaling (3716) \\
\hline Mcl-1 & 40 & $\begin{array}{l}\text { rabbit } \\
\text { polyclonal }\end{array}$ & $1: 1000$ & Cell Signaling (5453) \\
\hline $\begin{array}{l}\text { P-Akt } \\
(\text { Ser 473) }\end{array}$ & 60 & $\begin{array}{l}\text { rabbit } \\
\text { polyclonal }\end{array}$ & $1: 1000$ & Cell Signaling (9271) \\
\hline P-cJun (Ser 63) & 48 & $\begin{array}{l}\text { rabbit } \\
\text { polyclonal }\end{array}$ & $1: 500$ & Cell Signaling (9261) \\
\hline P-erk1 (Tyr 204) & 44 & $\begin{array}{l}\text { mouse } \\
\text { monoclonal }\end{array}$ & $1: 500$ & Santa Cruz (sc-7383) \\
\hline $\begin{array}{l}\text { P-JNK1/2 } \\
\text { (Thr } 183 \text { / Tyr 185) }\end{array}$ & $46 / 54$ & $\begin{array}{l}\text { rabbit } \\
\text { polyclonal }\end{array}$ & $1: 500$ & Cell Signaling (4668S) \\
\hline $\begin{array}{l}\text { P-S6 Kinase } \\
(\text { Ser } 235 \text { / 236) }\end{array}$ & 32 & $\begin{array}{l}\text { rabbit } \\
\text { polyclonal }\end{array}$ & $1: 1000$ & Cell Signaling (2211) \\
\hline PARP & $85 / 116$ & $\begin{array}{l}\text { mouse } \\
\text { monoclonal }\end{array}$ & $1: 1000$ & Calbiochem (AM30) \\
\hline PARP & $85 / 116$ & $\begin{array}{l}\text { rabbit } \\
\text { polyclonal }\end{array}$ & $1: 2000$ & Cell Signaling (9542) \\
\hline
\end{tabular}

Table 3.12: Secondary Antibodies

Name

HRP-coupled AffiniPure F(ab')2

fragment, anti-mouse $\operatorname{IgG}(\mathrm{H}+\mathrm{L})$

Goat anti-rabbit IgG-HRP
Cat.No.

711-036-152

sc-2004

\section{Company}

Jackson Immunoresearch, Europe, Newmarket, UK

Santa Cruz 


\subsection{Human cell culture}

Table 3.13: Human cell lines

\begin{tabular}{|c|c|c|}
\hline Name & Phenotype & Feature \\
\hline $24^{+B C l-x L}$ & epithelial & stably expressing \\
\hline & & Bcl-xL \\
\hline $24^{+}$HMLE & epithelial & $\begin{array}{l}\text { no selection } \\
\text { required }\end{array}$ \\
\hline $24^{+}$RAS & epithelial & selection with \\
\hline $\mathrm{MSP}^{\mathrm{Bcl-xL}}$ & mesenchymal & $\begin{array}{l}\text { Blasticidin } \\
\text { stably expressing } \\
\text { Bcl-xL }\end{array}$ \\
\hline MSP & mesenchymal & $\begin{array}{l}\text { no selection } \\
\text { required }\end{array}$ \\
\hline MSP RAS & mesenchymal & $\begin{array}{l}\text { selection with } \\
\text { Blasticidin }\end{array}$ \\
\hline
\end{tabular}

Table 3.14: Contents of MEGM medium for human cell culture (HMLE / HMLE RAS)

\begin{tabular}{|c|c|c|c|}
\hline Reagent & Company & Cat.No. & for 11 \\
\hline Blasticidin & Sigma Aldrich & 15205 & $5 \mathrm{ng} / \mathrm{ml}$ \\
\hline DMEM High Glucose & Invitrogen & 21063-045 & $250 \mathrm{ml}$ \\
\hline \multicolumn{4}{|l|}{ Phenol-Red Free } \\
\hline $\begin{array}{l}\text { F-12 Nutrient Mixture } \\
\text { (Ham), liquid }\end{array}$ & Invitrogen & $21765-029$ & $250 \mathrm{ml}$ \\
\hline \multirow[t]{7}{*}{ MEGM BulletKit } & Lonza & CC-3150 & 500ml MEGM \\
\hline & & & $0.004 \mathrm{ml} / \mathrm{ml} \mathrm{BPE}$ \\
\hline & & & 10ng/ml hEGF \\
\hline & & & $5 \mu \mathrm{g} / \mathrm{ml}$ human hydrocortisone \\
\hline & & & $5 \mu \mathrm{g} / \mathrm{ml}$ human insulin \\
\hline & & & $15 \mu \mathrm{g} / \mathrm{ml}$ Gentamicin \\
\hline & & & 7.5ng/ml Amphotericin \\
\hline human EGF & Sigma Aldrich & E9644 & $500 \mu 1$ \\
\hline human Hydrocortisone & Sigma Aldrich & H0888 & $500 \mu 1$ \\
\hline human Insulin & Sigma Aldrich & I9278 & $500 \mu 1$ \\
\hline
\end{tabular}

Table 3.15: Subculture reagents for human cell culture (HMLE / HMLE RAS)

\begin{tabular}{lrr} 
Reagent & Company & Cat.No. \\
\hline HEPES buffered Saline & Lonza & CC-5034 \\
Trypsin & Lonza & CC-5034 \\
TNS & Lonza & CC-5034
\end{tabular}

(Trypsin Neutralizing

Solution) 


\subsection{Mice}

Six week old female homozygous nude mice $(\mathrm{Nu} / \mathrm{Nu})$ were purchased from Janvier, Paris. Nude mice are laboratory mice with lacking a thymus, are unable to produce T-cells and are therefore immunodeficient. The genetic basis of these mice is a mutational disruption of the FOXN1 gene. All mice were kept in the animal facility of the Zentrale Tierexperimentelle Einrichtung at the University Medical Center Göttingen. Mice were handled according the restrictions of the German animal welfare act.

\subsection{Software}

Table 3.16: Software

Name Company

Adobe Photoshop CS5 Adobe Systems, San Jose, CA, United States

AxioVision 3.0 Zeiss

BioEdit v7.0.5

Tom Hall, Ibis Therapeutics, Isis Pharmaceuticals, Carlsbad, CA, United States

CFX Manager Software Bio-Rad

for qPCR cycler

Excel

Microsoft, Redmond, WA, United States

GraphPad Prism ${ }^{\circledR}$

GraphPad Software, Inc., CA United States

Guava Express Software

Millipore, Merck

INTAS lab ID

Intas Science Imaging Instruments

NanoDrop Software

Peqlab

Office Picture Manager

Microsoft, Redmond, WA, United States

UV imager software

Intas Science Imaging Instruments 


\section{Methods}

\subsection{Cell biology}

\subsubsection{Culturing of human cells}

Adherent human cells were cultured in coated cell culture flasks with filter caps at $37^{\circ} \mathrm{C}$, $5 \% \mathrm{CO}_{2}$ and a humidified atmosphere in MEGM media supplemented with 25\% DMEM high glucose phenol red free, 25\% F12 nutrient mixture, 0.004ml $/ \mathrm{ml} \mathrm{BPE,} 10 \mathrm{ng} / \mathrm{ml} \mathrm{hEGF}, 5 \mu \mathrm{g} / \mathrm{ml}$ human hydrocortisone, $5 \mu \mathrm{g} / \mathrm{ml}$ human insulin, $15 \mu \mathrm{g} / \mathrm{ml}$ Gentamicin and $7.5 \mathrm{ng} / \mathrm{ml}$ Amphotericin. For selection pressure and therefore preventing loss of the haRAS construct, culture medium for the haRAS transformed cells was supplemented with $5 \mathrm{ng} / \mathrm{ml}$ Blasticidin. For the subculture, cells were washed with HEPES buffered saline and detached by incubation with trypsin/EDTA at $37^{\circ} \mathrm{C}$. Subsequently, the enzymatic reaction of trypsin was stopped by adding Trypsin Neutralizing Solution (TNS). Afterwards, cells were centrifuged for $8 \mathrm{~min}$ at 900rcf and RT. The supernatant was aspirated and the cell pellet was dissolved in supplemented MEGM medium. The cells were reseeded at dilutions of 1:2.5 up to 1:5 every second day. For experiments, cell pellets were dissolved in Blasticidin-free medium, to have only one trigger during the cell treatments. The cell number was defined by counting with a Neubauer chamber and seeded at the respective density. All cell culture work was performed under sterile conditions.

\subsubsection{Freezing of cells and thawing of frozen cells}

For long-term storage, cells in a low passage were detached from a $175 \mathrm{~cm}^{2}$-size cell culture flask as described above. After centrifugation (8min, 900rcf, RT) the supernatant was aspired and the cell pellet was dissolved in $6 \mathrm{ml}$ cold FCS containing 10\% DMSO. The suspension was aliquoted in four cryo vials and stored at $-80^{\circ} \mathrm{C}$ for $24 \mathrm{~h}$ before the vials were transferred into liquid nitrogen. For thawing of the cells, the aliquots were taken from storage in the liquid nitrogen, were rapidly thawed and transferred into pre-warmed supplemented MEGM medium. Afterwards, the cells were centrifuged (8min, 900rcf, RT) and the pellets were resuspended in fresh pre-warmed medium. Subsequently, $24^{+}$HMLE (RAS, Bcl-xL) and MSP (RAS, Bcl-xL) cells were seeded into $25 \mathrm{~cm}^{2}$ - and $75 \mathrm{~cm}^{2}$-size cell culture flasks, respectively. Medium was changed after $24 \mathrm{~h}$ to remove dead cells. 


\subsubsection{Model system for EMT: HMLE and HMLE RAS cells}

\subsubsection{Generating the HMLE and HMLE RAS system}

The model systems for Epithelial Mesenchymal Transition (EMT) consisting of $24^{+} \mathrm{HMLE}$ (Human Mammary Large T-antigen immortalized Epithelial cells) and MSP (Mesenchymal Subpopulation) cells as well as $24^{+}$RAS and MSP RAS cells. We were kindly provided with these cells by Weinberg and colleagues (MIT Boston, USA). To create the model system for EMT, HMECs (Human Mammary Epithelial Cells) were obtained from reduction mammoplasty tissue samples from a healthy donor and were immortalized using retroviral transfection expressing the catalytic subunit of the human telomerase enzyme hTERT and the SV -40 large $\mathrm{T}$ antigen. Thereby, hTERT ensures maintenance of the telomers, while the SV-40 large $\mathrm{T}$ antigen is involved in the inactivation of the p53 and pRB pathways. To generate tumorigenic HMLEs the cells were transformed with the haRAS oncogene acquiring a constitutive mitogenic signal [112].

The Retroviral transfection was performed by Weinberg and colleagues (MIT Boston, USA) as previously described [112]. In short, the retroviral transfections were performed in series with drug selection to purify polyclonal-infected populations after each infection. First, amphotropic retroviruses were produced by co-transfection of $293 \mathrm{~T}$ cells with $8 \mu \mathrm{g}$ of the amphotropic packaging plasmid pCL-10A1 and the specified pBabe retroviral expression construct using calcium phosphate precipitation. After $28 \mathrm{~h}$ the viral supernatants were harvested and used to infect the HMECs applying $8 \mu \mathrm{g} / \mathrm{ml}$ polybrene. The transfection efficiency was proven by parallel infections with GFP-expressing retroviruses. To prepare Bcl2L1 overexpressing HMLE cells, the $24^{+}$HMLE were transfected with the specific pBABEzeo construct as described above. Infected cells were selected with $2 \mu \mathrm{g} / \mathrm{ml}$ puromycin, $200 \mu \mathrm{g} / \mathrm{ml}$ hygromycin, $200 \mu \mathrm{g} / \mathrm{ml}$ neomycin and $500 \mu \mathrm{g} / \mathrm{ml}$ zeomycin.

\subsubsection{Isolation of floating MSP cells from HMLE cultures}

To generate MSP cells from their parental $24^{+}$HMLE, limited trypsination of $24^{+}$HMLE was performed. First, the respective $24^{+}$HMLE cells were cultured as described before (4.1.1). However, after applying Trypsin the cells were incubated for max. $3 \mathrm{~min}$ at $37^{\circ} \mathrm{C}$ and $5 \% \mathrm{CO}_{2}$. Afterwards, the cells were forced to detach by rapping on the cell culture flask. The cell suspension containing only fast detached cells was transferred into a $15 \mathrm{ml}$ reaction tube and Trypsin was neutralized by adding TNS in the same amount. After centrifugation (8min, 900rcf, RT), the supernatant was removed and the pellet was resolved in supplemented 
MEGM medium and completely reseeded into a new cell culture flask. The process of limited trypsination was repeated at least three times every second day until the morphological change to mesenchymal cells was visible.

\subsubsection{Transient transfection with siRNA}

The selective knock down of target proteins was performed transiently by reverse transfection using siRNA (final concentration $12.5 \mathrm{nM}$ ). For 6-well plates two different solutions were prepared. Solution A contained $0.5 \mu$ l of siRNA $(50 \mu \mathrm{M})$ in supplemented MEGM medium $(250 \mu 1)$, whereas solution B contained $15 \mu$ l Lipofectamine 2000 in supplemented MEGM medium $(250 \mu \mathrm{l})$. After $5 \mathrm{~min}$ incubation the solutions were combined in 6-well plates and incubated for 20 to $45 \mathrm{~min}$ at RT. Meanwhile, the cells were adjusted to the defined cell concentrations $\left(1 \times 10^{5}\right.$ cells $\left./ \mathrm{ml}\right)$ depending on the experimental setup. The cell solution was added to the prepared transfection solution and mixed by swaying back and forth. The medium was changed after $24 \mathrm{~h}$ incubation at $37^{\circ} \mathrm{C}$ and $5 \% \mathrm{CO}_{2}$. Knock down was performed $24 \mathrm{~h}$ up to $48 \mathrm{~h}$ before treatment, depending on the experimental setup.

\subsubsection{Chemical treatment}

Cells were seeded in the respective density $24 \mathrm{~h}$ before chemical treatment. The medium was removed and fresh supplemented MEGM medium containing drugs in the indicated concentrations were added (Table 4.1). To treat the cells with chemotherapeutics in combination with inhibitors, the reagents were dissolved in DMSO and pre-diluted in supplemented MEGM medium, if necessary. Depending on the experimental outline the cells were treated with inhibitors in the presence or absence of chemotherapeutics for the respective time periods (Table 4.2). Control samples were treated with similar amounts of respective solvent.

Table 4.1: Concentrations of chemotherapeutics used for cell treatment

\begin{tabular}{lrrrr} 
Chemotherapeutic & $\begin{array}{r}\text { Stock } \\
\text { concentration }\end{array}$ & $\begin{array}{r}\text { Final } \\
\text { concentration }\end{array}$ & Solvent & Duration \\
\hline Carboplatin & $27 \mathrm{mM}$ & $0.75-1.5 \mathrm{mM}$ & $\mathrm{H} \mathrm{H}_{2} \mathrm{O}$ & $16 \mathrm{~h}$ \\
Cisplatin & $3.45 \mathrm{mM}$ & $5-40 \mu \mathrm{M}$ & $\mathrm{NaCl}$ & $8-16 \mathrm{~h}$ \\
Doxorubicin & $4 \mathrm{mM}$ & $200-500 \mathrm{nM}$ & $\mathrm{DMSO}$ & $24 \mathrm{~h}$ \\
Neocarcinostatin & $0.5 \mathrm{mg} / \mathrm{ml}$ & $150 \mathrm{ng} / \mathrm{ml}$ & $\mathrm{MES}$ buffer & $20 \mathrm{~h}$
\end{tabular}


Table 4.2: Concentrations of inhibitors for cell treatment

\begin{tabular}{llrr} 
Inhibitor & Target & $\begin{array}{r}\text { Stock } \\
\text { concentration }\end{array}$ & $\begin{array}{r}\text { Use } \\
\text { concentration }\end{array}$ \\
\hline 17AAG & HSP90 & $170 \mathrm{mM}$ & $5 \mu \mathrm{M}$ \\
17DMAG & HSP90 & $5 \mathrm{mM}$ & $5 \mu \mathrm{M}$ \\
Gossypol & Bcl-xL & $100 \mathrm{mM}$ & $6-30 \mu \mathrm{M}$ \\
LY294002 & Akt & $10 \mathrm{mM}$ & $50 \mu \mathrm{M}$ \\
Rapamycin & mTOR & $1 \mathrm{mg} / \mathrm{ml}$ & $50 \mathrm{nM}$ \\
SB203580 & p38 & $10 \mathrm{mM}$ & $10 \mu \mathrm{M}$ \\
SB431542 & TGF & $10 \mathrm{mM}$ & $10 \mu \mathrm{M}$ \\
SB600125 & JNK & $10 \mathrm{mM}$ & $10 \mu \mathrm{M}$ \\
U0126 & Mek & $25 \mathrm{mM}$ & $25 \mu \mathrm{M}$
\end{tabular}

\subsubsection{Proliferation Assay}

Cell proliferation was analyzed using the Celigo ${ }^{\circledR}$ Cytometer. A direct cell counting was performed by defining the cell number per well on the basis of contrast in bright field light microscopy. For the measurement, the cells were seeded into 12 -well plates $\left(1.25 \times 10^{4} / \mathrm{ml}\right)$ in duplicates or triplicates for every experimental setting. $24 \mathrm{~h}$ after seeding the treatment was applied to the cells. Cell counting was performed once a day after adding fresh MEGM medium for the respective time periods.

\subsubsection{Flow cytometry}

For analyzing caspase activation after chemotherapeutic treatment, the cells were analyzed by flow cytometry. To define healthy, early and late apoptotic cells, the Guava® Multi Caspase FAM Kit was used. This approach uses an inhibitor that binds specifically to the active site of caspases (so called "Caspase Reagent"). The inhibitor is conjugated to a fluorescent dye. The inhibitor is colligated to activated caspases with a fluoromethyl ketone group. The inhibitor is able to traverse the cell membrane. Once bound to an activated caspase, the inhibitor is trapped in the cell while unbound inhibitors diffuse out of the cell and are washed out during the washing step. The measured fluorescence signal is proportional to the number of active caspases that were represent in the cells at the beginning of the assay [113]. The implementation was done according the manufacturer's instructions. In short, the supernatants of treated cells were harvested separately by centrifugation ( $8 \mathrm{~min}, 900 \mathrm{rcf}, \mathrm{RT}$ ) while attached cells were harvested by trypsination (4.1.1). Afterwards, cells of the respective samples were combined and adjusted to a cell number of $1 \times 10^{4}$ to $5 \times 10^{5}$ cells $/ \mathrm{ml}$ in supplemented MEGM medium. The cells were mixed with the provided caspase working reagent and incubated for $1 \mathrm{~h}$ at $37^{\circ} \mathrm{C}$ 
and $5 \% \mathrm{CO}_{2}$. After washing the cells with apoptosis buffer (3 times, 5min, 300g, RT), 7Aminoactinomycin (7-AAD) was added to the separate samples. 7-AAD intercalates into double-stranded DNA. 7-AAD is excluded by viable cells, however it can penetrate through membranes of dying and dead cells. It is therefore possible to simultaneously evaluate membrane integrity and cell viability. The samples were measured with the Guava ${ }^{\circledR}$ EasyCyte ${ }^{\mathrm{TM}}$ flow cytometer by counting 5.000 events/sample.

\subsubsection{Immunohistochemistry}

To assess Bcl-xL level in human breast cancer, human tissues were analyzed for their Bcl-xL levels and mesenchymal character by immunohistochemistry and subsequent color intensity quantification in collaboration with Andreas Scheel, Institute for Pathology Nordhessen. Human tissue fixed in PBS-buffered formaline 4\% was embedded in paraffin. $1.5 \mu \mathrm{m}$ sections were cut and treated with boric-acid/EDTA buffer for antigen-retrieval. Bcl-xL and Cytokeratin 8 were stained using a specific primary antibody and UltraView DAB IHC detection kit on a BenchMark XT autostainer according to the manufacturer's instructions. For NCadherin staining, a specific primary antibody together with the UltraView Universal Alkaline Phosphatase Red Detection Kit was used.

The slides were digitized with a ScanScope XT at 400x magnification and color intensity quantification was achieved with the ImageJ software [114] by manually selecting the areas of interest and performing a color deconvolution [115]. All experiments were in accordance with the local ethics committee.

\subsection{Molecular biology}

\subsubsection{RNA standard techniques}

\subsubsection{Isolation of total RNA}

Total RNA was isolated using Trizol and used for subsequent reverse transcription (4.2.1.3) and quantification by real time PCR (4.2.1.4). Cells in 6-well plates were washed with icecold PBS followed by adding $1 \mathrm{ml}$ Trizol per sample. The samples were incubated for $5 \mathrm{~min}$ at RT for cell lysis. Afterwards, $200 \mu \mathrm{l}$ chloroform per $1 \mathrm{ml}$ Trizol was added. The samples were shaken, incubated for $3 \mathrm{~min}$ at RT and centrifuged to separate the different phases $(15 \mathrm{~min}$, $\left.12.000 \mathrm{~g}, 4^{\circ} \mathrm{C}\right)$. The RNA containing aqueous upper phase was transferred into another eppen- 
dorf tube and purified by precipitation with $500 \mu 1$ isopropanol per $1 \mathrm{ml}$ Trizol. The samples were shaken and centrifuged $\left(10 \mathrm{~min}, 12.000 \mathrm{~g}, 4^{\circ} \mathrm{C}\right)$. Subsequently, the pellet was washed with $75 \% \mathrm{EtOH}\left(5 \mathrm{~min}, 7.500 \mathrm{~g}, 4^{\circ} \mathrm{C}\right)$. To remove any protein contamination, the pellet was dissolved in $30 \mu \mathrm{l}$ nuclease free $\mathrm{H}_{2} \mathrm{O}$ and precipitated with $300 \mathrm{mM} \mathrm{NaAc}$ and $70 \% \mathrm{EtOH}$ (15min, 14.000rpm, $4^{\circ} \mathrm{C}$ ). Afterwards, the RNA pellet was washed with $70 \%$ EtOH (5min, 14.000rpm, $4^{\circ} \mathrm{C}$ ) and air-dried. The RNA was dissolved in $30 \mu \mathrm{l}$ nuclease free $\mathrm{H}_{2} \mathrm{O}$ and heated for $5 \mathrm{~min}$ at $55^{\circ} \mathrm{C}$. The RNA was stored at $-80^{\circ} \mathrm{C}$.

\subsubsection{Quantification of RNA}

RNA concentration was measured with a NanoDrop spectrophotometer. To determine the concentration, the absorbance (A) at $260 \mathrm{~nm}$ was used while ratios of $\mathrm{A}_{260} / \mathrm{A}_{230}$ and of $\mathrm{A}_{260} / \mathrm{A}_{280}$ were used as indicators for the purity of the isolated RNA. Purified RNA should display values from 1.9 to 2.0 for of $\mathrm{A}_{260} / \mathrm{A}_{230}$ and 2.0 to 2.1 for of $\mathrm{A}_{260} / \mathrm{A}_{280}$. For distinct deviating values, the RNA was purified again (4.2.1.1).

\subsubsection{Reverse transcription}

For preparing cDNA, purified RNA (4.2.1.1) was reverse transcribed by using the reverse transcriptase M-MuLV. $1 \mu \mathrm{g}$ of the respective RNA was incubated with $4 \mu 1 \mathrm{dNTP}$ mix (2.5mM of each deoxyribonucleotide triphosphate, i.e. dATP, dCTP, dGTP and dTTP) and $2 \mu 1$ primer which consisted of a combination of anchored oligo-dT primers (using $50 \mu \mathrm{M}$ $\mathrm{dT}_{23} \mathrm{VN}$ ) and random nonamer primers (using $15 \mu \mathrm{M}$ nonamer primers). Additionally, nuclease free water to a total volume of $16 \mu 1$ was added and the reagent mix was incubated for $5 \mathrm{~min}$ at $70^{\circ} \mathrm{C}$ in the PCR cycler. Afterwards, $4 \mu \mathrm{l}$ of a master mix was added to every sample. Every reaction mix was composed of the following reagents:

\begin{tabular}{lll}
$2 \mu 1$ & reaction buffer & $10 \mathrm{x}$ \\
$0.25 \mu 1$ & RNase inhibitor & $(10 \mathrm{U})$ \\
$0.125 \mu 1$ & M-MuLV Reverse Transcriptase & $(25 \mathrm{U})$ \\
$1.625 \mu 1$ & RNase free water & \\
\hline
\end{tabular}

$4 \mu 1$ per sample

Incubation: $\quad$ Reverse transcription $\quad 42^{\circ} \mathrm{C} \quad 60 \mathrm{~min}$ Inactivation of the enzyme $95^{\circ} \mathrm{C} \quad 5 \mathrm{~min}$ 
To exclude contaminations with genomic DNA, a reaction without the reverse transcriptase was prepared for every RNA sample. Subsequently, the reactions were diluted with $30 \mu 1 \mathrm{nu}-$ clease free water to a total volume of $50 \mu 1$ and stored at $-20^{\circ} \mathrm{C}$.

\subsubsection{Quantitative real time PCR}

To analyze gene expression patterns a quantitative real time PCR was performed based on Ishiguro et al. [116]. All samples were analyzed in triplicates and samples without reverse transcriptase as well as a sample without cDNA were used as controls. Every reaction mix was composed of the following reagents:

\begin{tabular}{lll}
$14 \mu 1$ & qRT-PCR reaction mix & $25 \mathrm{x}$ \\
$0.075 \mu 1$ & primer forward & $(100 \mu \mathrm{M})$ \\
$0.075 \mu 1$ & primer reverse & $(100 \mu \mathrm{M})$ \\
$9.85 \mu 1$ & RNase free water & \\
$1.0 \mu 1$ & cDNA \\
\hline $25 \mu 1$ per sample
\end{tabular}

The qRT-PCR was done in 96-well plates and covered with a clear foil. The following program was used:

$\left.\begin{array}{lll}\text { Denaturation } & 95^{\circ} \mathrm{C} & 2 \mathrm{~min} \\ \text { Denaturation } & 95^{\circ} \mathrm{C} & 15 \mathrm{~s} \\ \text { Annealing } & 60^{\circ} \mathrm{C} & 15 \mathrm{~s} \\ \text { Elongation } & 72^{\circ} \mathrm{C} & 30 \mathrm{~s}\end{array}\right\} \quad 40 \mathrm{x}$

The fluorescence dye SYBR-Green is able to intercalate into double-stranded DNA. The fluorescence of each sample was measured after each cycle at the end of the elongation step. It is therefore possible to make a proposition about the amplification of the cDNA. Semiquantitative analysis of mRNA expression was conducted by measuring the $C_{t}$ values of the reference gene $36 \mathrm{~B} 4$ and the gene of interest. $\mathrm{C}_{\mathrm{t}}$ values were defined by setting a threshold within the exponential slope of the amplified product. To determine the relative gene expression of the analyzed target genes, the $\Delta \Delta \mathrm{C}_{\mathrm{t}}$ method was used:

$$
\Delta \Delta \mathrm{C}_{\mathrm{t}}=\frac{\mathrm{C}_{\mathrm{t}}(\text { reference gene, untreated })-\mathrm{C}_{\mathrm{t}}(\text { target gene, untreated })}{\mathrm{C}_{\mathrm{t}}(\text { reference gene, treated })-\mathrm{C}_{\mathrm{t}}(\text { target gene, treated })}
$$




\subsubsection{TaqMan® MicroRNA assay}

The TaqMan® MicroRNA assay was used to detect and quantify mature microRNAs (miRNA).

\subsubsection{Isolation of microRNA}

To isolate RNA for miRNA analysis, seeded cells were allowed to attach overnight, then washed with PBS and subsequently lysed by adding $1 \mathrm{ml}$ Trizol to every 6-well. After an incubation of $10 \mathrm{~min}$ at RT, $200 \mu \mathrm{l}$ Chloroform per $1 \mathrm{ml}$ Trizol was added and the samples were centrifuged (30min, $12.000 \mathrm{rcf}, 4^{\circ} \mathrm{C}$ ). The RNA containing aqueous upper phase was transferred into a new reaction tube and purified by precipitation with three times of absolute $\mathrm{EtOH}$ of the recovered volume. The samples were incubated at $-20^{\circ} \mathrm{C}$ overnight for precipitation and afterwards centrifuged (30min, $10.000 \mathrm{rcf}, 4^{\circ} \mathrm{C}$ ). The pellet was washed with $70 \%$ $\mathrm{EtOH}\left(5 \mathrm{~min}, 12.000 \mathrm{rcf}, 4^{\circ} \mathrm{C}\right)$. To remove protein contaminations, the pellet was dissolved in $100 \mu \mathrm{l}$ nuclease free $\mathrm{H}_{2} \mathrm{O}$ and precipitated with $2 \mathrm{M} \mathrm{NaAc}$ and $80 \% \mathrm{EtOH}$ for $30 \mathrm{~min}$ at $-20^{\circ} \mathrm{C}$ and subsequent centrifugation (30min, $14.000 \mathrm{rpm}, 4^{\circ} \mathrm{C}$ ). Afterwards, the RNA pellet was washed with $80 \% \mathrm{EtOH}\left(5 \mathrm{~min}, 14.000 \mathrm{rpm}, 4^{\circ} \mathrm{C}\right.$ ) and air-dried. The RNA was dissolved in $30 \mu \mathrm{l}$ nuclease free $\mathrm{H}_{2} \mathrm{O}$ and heated for $5 \mathrm{~min}$ at $55^{\circ} \mathrm{C}$. The RNA was stored at $-80^{\circ} \mathrm{C}$.

\subsubsection{MicroRNA specific reverse transcription}

For preparing miRNA specific cDNA, the isolated miRNA (4.2.2.1) was reversely transcribed using miRNA specific primer and the reverse transcriptase Multiscribe. miRNA concentration was measured as described before (4.2.1.2) and subsequently adjusted to $2 \mathrm{ng} / \mathrm{ml}$ with nuclease-free $\mathrm{H}_{2} \mathrm{O}$. Every reaction mix was composed of the following reagents:

$\begin{array}{ll}0.15 \mu 1 & \text { dNTPs } \\ 1.5 \mu 1 & \text { RT buffer } \\ 0.188 \mu 1 & \text { RNase inhibitor } \\ 4.162 \mu 1 & \text { RNase free water } \\ 3 \mu 1 & 5 x \text { primer (for the corresponding miRNA) }\end{array}$

$9 \mu 1$ per sample

$1 \mu \mathrm{l}$ reverse transcriptase Multiscribe and $5 \mu \mathrm{l}$ RNA $(2 \mathrm{ng} / \mathrm{ml})$ were added to every reaction mix. A reaction mix with water instead of the reverse transcriptase Multiscribe was used as 
control. Subsequently, the reactions were diluted at least 1:15 with nuclease free $\mathrm{H}_{2} \mathrm{O}$ and stored at $-20^{\circ} \mathrm{C}$.

PCR program:

\begin{tabular}{lrr} 
Step & time [min] & temperature $\left[{ }^{\circ} \mathbf{C}\right]$ \\
\hline 1 & 30 & 16 \\
2 & 30 & 42 \\
3 & 5 & 85
\end{tabular}

\subsubsection{TaqMan® PCR}

To quantify miRNA expression a TaqMan ${ }^{\circledR}$ PCR was performed according to the manufacturer's instructions. All samples were analyzed in triplicates and samples without RT product (4.2.2.2) were used as controls. The following components were added per sample:

\begin{tabular}{ll}
$10 \mu 1$ & TaqMan 2x universal PCR Master Mix \\
$0.9 \mu 1$ & 20x TaqMan Assay \\
$7.77 \mu 1$ & RNase free water \\
$1.33 \mu 1$ & RT product $(4.2 .2 .2)$ \\
\hline $20 \mu 1$ per sample
\end{tabular}

The TaqMan ${ }^{\circledR}$ PCR was done in 96-well plates and covered with a clear foil. For quantification all samples were normalized to the nucleolar RNA U6.

TaqMan®-PCR program:

$\left.\begin{array}{lll}\text { AmpliTaq Gold enzyme activation } & 95^{\circ} \mathrm{C} & 10 \mathrm{~min} \\ \text { Denaturation } & 95^{\circ} \mathrm{C} & 15 \mathrm{~s} \\ \text { Annealing } & 60^{\circ} \mathrm{C} & 60 \mathrm{~s}\end{array}\right\} 45 \mathrm{x}$

To analyze the TaqMan ${ }^{\circledR}$ PCR, a threshold was set within the exponential slope of the amplified product to define $\mathrm{C}_{\mathrm{t}}$ values. The relative amount of miRNAs was determined by using the $\Delta \Delta \mathrm{C}_{\mathrm{t}}$ method: 


$$
\Delta \Delta \mathrm{C}_{\mathrm{t}}=\frac{\mathrm{C}_{\mathrm{t}}(\mathrm{U} 6, \text { untreated })-\mathrm{C}_{\mathrm{t}}(\text { target miRNA, untreated })}{\mathrm{C}_{\mathrm{t}}(\mathrm{U} 6, \text { treated })-\mathrm{C}_{\mathrm{t}}(\text { target miRNA, treated })}
$$

\subsubsection{Gene expression analysis using microarray}

For comparison of total mRNA expression levels of $24^{+}$HMLE and MSP cells, a whole genome microarray was performed. For every cell line, three independent samples were prepared. Total RNA was isolated using Trizol (4.2.1.1) and delivered to the transcriptome analysis laboratory (TAL) Göttingen. TAL controlled the RNA quality, measured the concentration and used 200ng of total RNA for reverse transcription into cDNA. Afterwards, the cDNA was transcribed into antisense RNA with a master mix containing the sample cDNA, dNTPs and T7 RNA polymerase. To label the sequence, Cy3-CTP was added to the transcription master mix as well. The labeled and purified antisense RNA was hybridized to the microarray slide, where the antisense RNA binds to complementary sequences printed on the array. The array slides were read out by laser application, measuring the fluorescence intensity of the Cy3 excitation. Strong emission at a particular RNA spot is correlated with a high expression of the specific gene in the sample. Subsequently, the data were analyzed by Lennart Opitz (TAL, Göttingen). For analyses, data of the three independent samples of each cell line were combined and the MSP data were analyzed relatively to the $24^{+}$HMLE data. The threshold of differentially regulated mRNA expression in MSP cells compared to $24^{+} \mathrm{HMLE}$ was set to 2 and the induction was calculated as $\log 2$ values. Positive values indicate an up-regulation and negative ones a down-regulation of gene expression in MSP cells compared to $24^{+}$HMLE.

\subsection{Biochemistry and immunological methods}

\subsubsection{Immuno-Slot-Blot assay}

To quantify the damaged DNA after Cisplatin treatment, the Cisplatin-adducts that were formed between the platinum atom and DNA after Cisplatin treatment were measured. DNA was firstly extracted with NucleoBond ${ }^{\circledR}$ AXG columns. Subsequently, DNA was analyzed for formed adducts by performing an Immuno-Slot-Blot assay based on Nehls et al. [118] in collaboration with Jürgen Thomale, University of Duisburg-Essen. 


\subsubsection{Extraction of DNA using NucleoBond® AXG columns}

For DNA extraction of Cisplatin treated cells NucleoBond® AXG columns were used according to the manufacturer's instructions. In short, the cells were harvested by trypsination (4.1.1) and adjusted to $1 \times 10^{7} / \mathrm{ml}$ in PBS. The cells were disrupted by adding a specific lysis buffer and lysates were digested with Proteinase $\mathrm{K}$ for $1 \mathrm{~h}$ at $50^{\circ} \mathrm{C}$. The samples were applied to the NucleoBond ${ }^{\circledR}$ AXG columns and entered the resin by gravity flow. DNA was eluted twice with $5 \mathrm{ml}$ elution-buffer after three washing steps. Afterwards the DNA was precipitated with isopropanol (0.7 times) and incubated for $1 \mathrm{~h}$ at RT. After centrifugation (30min, $12.000 \mathrm{rpm}, 4^{\circ} \mathrm{C}$ ) the pellets were washed with $70 \% \mathrm{EtOH}$ and dried at $55^{\circ} \mathrm{C}$. Finally, the pellets were dissolved in $95 \mu 1$ nuclease free water and stored at $-20^{\circ} \mathrm{C}$.

\subsubsection{Immuno-Slot-Blot}

For analysis of the platinum atom-DNA intrastrand crosslinks, an Immuno-Slot-Blot was performed by Jürgen Thomale, University of Duisburg-Essen. The extracted DNA from the Cisplatin treated cells (4.3.1.1) was first denatured for $10 \mathrm{~min}$ at $95^{\circ} \mathrm{C}$. The samples were subsequently put on ice and diluted with $100 \mu \mathrm{l}$ ice-cold ammonium acetate (2M). For the ImmunoSlot-Blot, a nitrocellulose Hybond membrane was soaked in $1 \mathrm{M}$ ammonium acetate, the slotblot apparatus was assembled and $200 \mu 1$ sample was applied onto the membrane. Afterwards, each slot was washed twice with $1 \mathrm{M}$ ammonium acetate. Subsequently, the membrane was removed from the apparatus. The membrane was soaked with 5xSSC for $5 \mathrm{~min}$ and washed with $\mathrm{H}_{2} \mathrm{O}$. Afterwards, the membrane was dried and the DNA was fixed by heating ( $2 \mathrm{~h}$, $80^{\circ} \mathrm{C}$ ). Unspecific antibody binding sites were blocked with blocking buffer for $2 \mathrm{~h}$. Afterwards, the membrane was incubated with the lesion specific antibody R-C18 $(0.2 \mu \mathrm{g} / \mathrm{ml})$ in blocking buffer for $1 \mathrm{~h}$ at RT [14]. Thereafter, the membrane was washed twice in PBS/0.2\% Tween for 10min and then exposed to the anti-rat HRP-linked secondary antibody (1:2000 in blocking buffer) for $1 \mathrm{~h}$ at RT. Afterwards the membrane was washed again and covered with 1:1 mixed ECL solution for a few minutes. The luminescence was detected using an ECLreader. 


\subsubsection{Immunoblot analysis}

\subsubsection{Generation of cell lysates for SDS-PAGE analysis}

Adherent cells were treated or transfected in 6-well plates for immunoblot analysis. To prepare cell lysates for subsequent analysis by Sodium Dodecyl Sulfate PolyAcrylamide Gel Electrophoresis (SDS-PAGE), the supernatants of each well was collected in eppendorf tubes. Afterwards, the samples were centrifuged $\left(5 \mathrm{~min} ; 3000 \mathrm{rct} ; 4^{\circ} \mathrm{C}\right)$. The attached cells in the 6well plate were washed once with ice-cold PBS and covered with $85 \mu 1$ lysis buffer (containing protease inhibitors). The cells were scraped off and combined with the respective cellpellets of the supernatants. The protein concentration was measured with Nanodrop (4.3.2.2) and adjusted to the lowest protein concentration of the experiment by adding $6 \%$ Laemmli and lysis buffer $(20 \mu 1$ total volume). For protein denaturation, the samples were boiled for $3 \mathrm{~min}$ at $95^{\circ} \mathrm{C}$ and finally sonicated for $10 \mathrm{~min}$. The samples were stored at $-20^{\circ} \mathrm{C}$.

\subsubsection{Determination of protein concentrations}

Protein concentration was measured using the BCA Protein Assay Kit based on the biuret reaction according to the manufacturer's instructions. In short, a master mix of $4 \%$ cupric sulfate and BCA solution (1:50) was added to the protein lysates (1:20) and incubated for 30min at $37^{\circ} \mathrm{C}$. Afterwards the concentration was measured with Nanodrop and adjusted to the lowest protein concentration in the experiment.

\subsubsection{Separation of proteins by SDS-PAGE}

To separate proteins under denaturing conditions, SDS-PAGE was performed as developed by Shapiro et al. in 1967 and Laemmli in 1970 [119; 120]. Separation of the proteins relies on their electrophoretic mobility. Prior the electrophoresis the proteins are boiled in presence of sodium dodecyl sulfate (SDS). Thus, SDS coats the proteins which results in a negative overall charge, so that proteins move to the anode if an electric field is applied. Thereby, proteins with lower weight travel faster through the pores of the gel, while large proteins are slower. The stacking gel contains large pores and the proteins are forming a stack between the leading chloride ions and the trailing ion Glycine. Afterwards proteins reach the resolving gel. Its $\mathrm{pH}$ is higher than that of the stacking gel and contains smaller pores. Thus, the proteins are separated according to their molecular weight. Depending on the molecular weight of the investi- 
gated proteins, the different percentages of acrylamide/ bisacrylamide are used within the resolving gel. Details on the composition of the gels can be found in the following overview:

\begin{tabular}{lrr} 
Chemical & Stacking gel & Resolving gel \\
\hline Acrylamide/ bisacrylamide & $5 \%$ & $8-12 \%$ \\
Tris, pH 6.8 (1M) & $126 \mathrm{mM}$ & - \\
Tris, pH 8.8 (1.5M) & - & $375 \mathrm{mM}$ \\
SDS (10\%) & $0.1 \%$ & $0.1 \%$ \\
APS (10\%) & $0.1 \%$ & $0.1 \%$ \\
TEMED & $0.3 \%$ & $0.4 \%$
\end{tabular}

First the resolving gel was casted between two glass plates, which were separated by two spacers. The gel was topped with isopropanol during the polymerization to prevent air contact and dehydration. The polymerized gel was rinsed with water and the stacking gel was filled on top. To form separated slots for the application of the protein lysates, a comb with 10 or 15 teeth was fit in the liquid gel.

For electrophoresis, the protein samples were boiled for $3 \mathrm{~min}$ at $95^{\circ} \mathrm{C}$ and $18 \mu \mathrm{l}$ sample were applied to the pockets of the stacking gel. The electrophoresis was performed at $20 \mathrm{~mA}$ per gel.

\subsubsection{Immunoblotting}

The proteins that were separated by SDS-PAGE (4.3.2.3) were visualized using a tankblot technique based on Bittner et al. [121]. Thereby, proteins from the SDS gel are transferred to a nitrocellulose membrane whereon the proteins are detected with a primary antibody specific for the target protein and a secondary antibody that is coupled to horseradish peroxidase (HRP). Application of the substrate luminol that is oxidized by HRP then results in luminescence. The emitted light is detected by a camera and thus protein bands are visualized. For the wet blot, the sponges, Whatman paper and nitrocellulose membrane with $0.2 \mu \mathrm{m}$ pore size were soaked with transfer buffer. Then the SDS gel was placed in a stack. Therefore, two sponges were assembled at the anode side, followed by three Whatman papers, the nitrocellulose membrane and the SDS gel, followed by three Whatman papers and one sponge at the cathode side. The stack was placed into a blotting chamber, which was filled with transfer buffer and the blotting was performed at $100 \mathrm{~V}$ for $120 \mathrm{~min}$ at $4^{\circ} \mathrm{C}$. Afterwards, the transfer chamber was disassembled and the nitrocellulose membrane was washed with water for a few seconds. Then, unspecific antibody binding sites of the membrane were blocked with blocking buffer for at least 30min at RT. Subsequently, the membrane was put into $5 \mathrm{ml}$ antibody 
solution and incubated overnight at $4^{\circ} \mathrm{C}$. After washing three times with TBS/T and three times with blocking buffer, the membrane was exposed to the respective secondary antibody (in $5 \mathrm{ml}$ blocking buffer) for at least $1 \mathrm{~h}$ at RT. Afterwards the membrane was washed again as before. To detect protein bands, the SuperSignal ${ }^{\circledR}$ Millipore solution Luminol and stable peroxide solution were mixed 1:1 and applied onto the membrane. The luminescence was detected using a Chemocam HR 163200 imager. Weak signals were detected with the more sensitive substrate solution SuperSignal West Femto Maximum.

\subsection{MSP RAS injection in mice and HSP90 inhibition}

Xenograft experiments were performed to assess the inhibition of HSP90 in MSP RAS cells derived tumors in mice. Six-week-old nude mice were anesthetized with 3-4\% Isofluran, followed by $\gamma$-irradiation $(1.87 \mathrm{~Gy} / \mathrm{min})$. MSP RAS cells have been grown to $70 \%$ confluence and were trypsinized as described previously (4.1.1). Subsequently, cells were resuspended in antibiotic free MEGM medium at 1x106 cells per 100 $\mu 1$. Cell suspension was diluted 1:1 with Matrigel $^{\mathrm{TM}}$ immediately before injection in the flank region of the nude mice. Tumor growth was monitored daily using a caliper. In the phase of exponential tumor growth, approximately three weeks after injection and tumor volume of $200 \mathrm{~mm}^{3}$, xenograft mice were randomly assigned to two groups. 17DMAG was dissolved in sterile $\mathrm{H} 2 \mathrm{O}$ and stored at $-20^{\circ} \mathrm{C}$ until use. For daily intraperitoneal administration of $0.06 \mathrm{mg} / 10 \mu 1$ 17DMAG $(200 \mu 1 / \mathrm{mice}), 17 \mathrm{DMAG}$ was diluted 1:1 with $10 \%$ glucose solution.

\subsection{Statistical analysis}

Statistical significances were defined by the unpaired, two-tailed student's t-test using Microsoft Excel. Results were considered as significant for p-values below 0.05. In figures, asterisks indicate resulting $\mathrm{p}$-values as follows: $* \mathrm{p}<0.05$, $* * \mathrm{p}<0.01$, $* * * \mathrm{p}<0.001$. n.s. $=$ not significant. $\mathrm{n}$ in figure legends indicates the number of independent experiments 


\section{Results}

\subsection{EMT results in chemotherapeutic resistance}

\subsubsection{HMLE (RAS) system as a convenient model for EMT}

It is well established that Epithelial Mesenchymal Transition (EMT) is not only involved in embryogenic development but rather plays an important role in tumor metastasis as well as in chemotherapeutic resistance. In different tumor types typical markers for EMT occur and were found to be accompanied by resistances to different chemotherapeutic drugs [105]. To investigate whether EMT results in chemotherapeutic resistance, a convenient model system was an absolute need. We therefore were kindly provided with the HMLE and HMLE RAS system by Weinberg and colleagues (MIT Boston, USA). In this system, $24^{+}$HMLE and $24^{+}$HMLE RAS cells display the parental cell population in their original epithelial cell shape and behavior, while MSP and MSP RAS cells are a mesenchymal subpopulation originating from the 24+HMLE or 24+HMLE RAS, respectively, and display properties of cells upon EMT $[110 ; 111 ; 112]$.

To validate the respective markers for EMT in HMLE and HMLE RAS we made use of qRTPCR and micro array analysis. mRNA of untreated cells was isolated and gene expression of morphological markers like Vimentin and E-Cadherin as well as the cell surface marker CD24 and genes that are directly affected by EMT-induced signalling pathways were identified. As expected, the mesenchymal marker Vimentin is up-regulated in MSP (RAS) cells, while the epithelial marker E-Cadherin is decreased. Concomitant with EMT, Zeb1, Zeb2 and Twist gene expression were found to be increased in MSP and MSP RAS together with the Wnt-target genes Axin2, MMP7 and cMyc, which are distinctively up-regulated by activated Wnt signaling pathway (Figure 5.1A, C). Additionally, micro array analysis resulted in 812 differentially regulated genes implicating the substantial alteration of morphological and cellular processes associated with EMT (Figure 5.1B, appendix).

In conclusion, the HMLE as well as HMLE RAS system display characteristic markers for cells before and upon EMT. Not only the morphological change from epithelial to mesenchymal phenotype, but also cellular signalling pathways that are affected by EMT seemed to be active in MSP and MSP RAS. This is reflected by the up-regulation of Wnt-responsive genes (Axin2, MMP7, cMyc) and TGF $\beta /$ Smad regulated genes (Zeb1, Zeb2). These results suggest the HMLE and HMLE RAS systems as an acceptable model for EMT. 


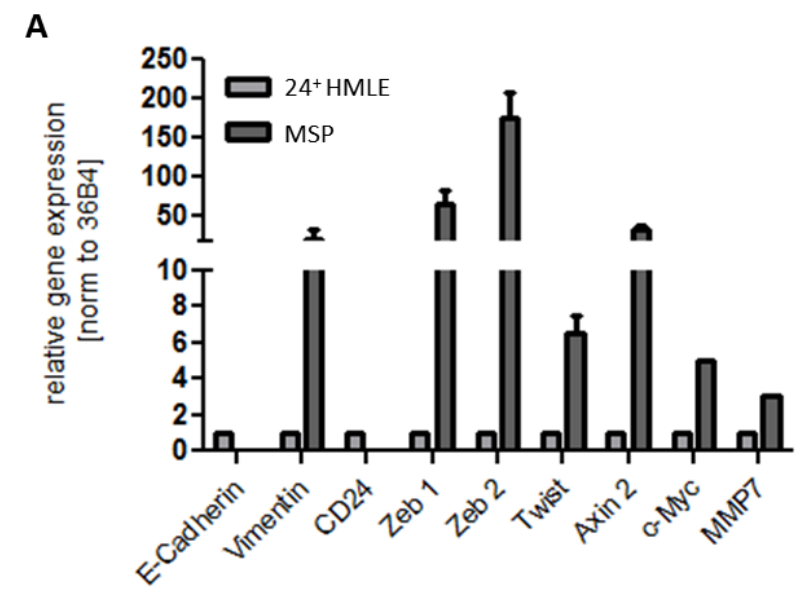

B

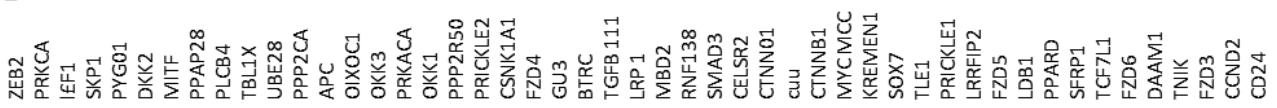

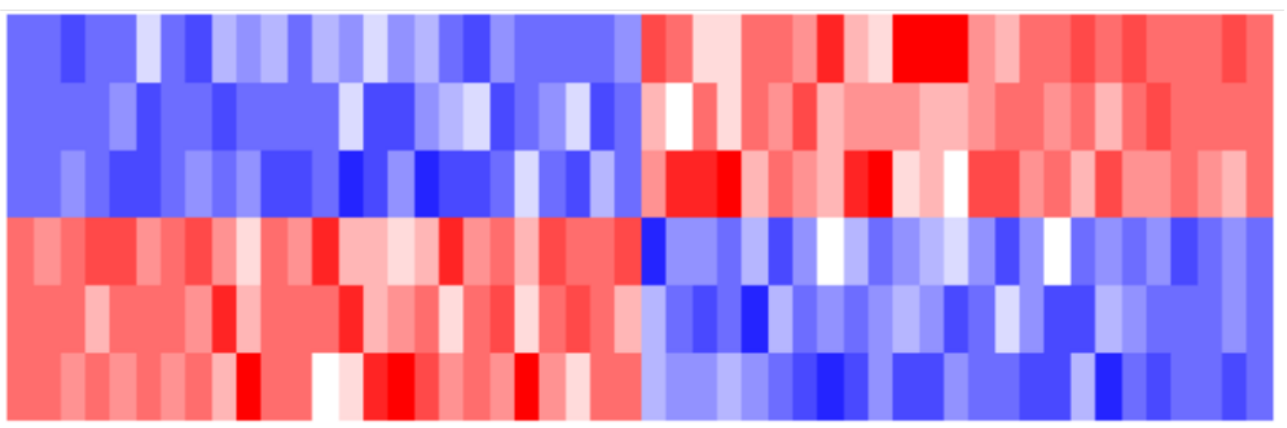

\#1 MSP

\#2 MSP

\#3 MSP

\#1 24 +HMLE

\#2 24 +HMLE

\#3 $24{ }^{+} \mathrm{HMLE}$
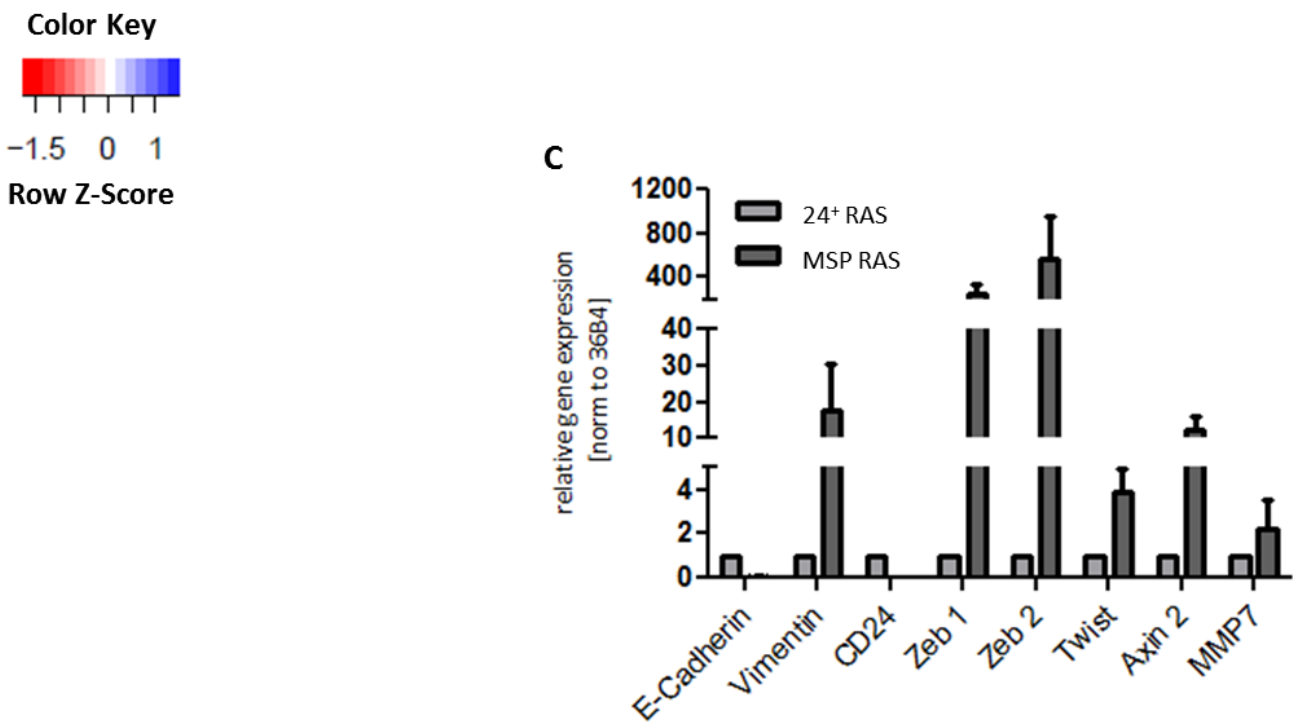

Figure 5.1: Validation of differentially regulated EMT markers in cells before and upon EMT.

(A, C) HMLE (A) and HMLE RAS cells (C) were harvested 24h after seeding. mRNA was isolated and mRNA level of the indicated genes were analyzed by qRT-PCR. mRNA level were normalized to 36B4. Data show mean \pm SD of $n=3$. (B) Micro array analysis was performed in untreated $24^{+}$HMLE and MSP cells to contrast EMT regulated genes. The heat map represents the top 50 deregulated genes in MSP cells relative to the $24^{+}$HMLE cells. By setting a threshold of differentially regulated mRNA expression in $24^{+}$HMLE compared to MSP cells to 2-fold, 812 genes were found to be differentially regulated upon EMT. 


\subsubsection{In response to Cisplatin no apoptosis is detectable in MSP and MSP RAS cells}

To address the question, whether EMT affects the response to chemotherapy HMLE and HMLE RAS cells were exposed to Cisplatin for $8 \mathrm{~h}$ or $16 \mathrm{~h}$. Thereupon, cells were harvested and apoptotic markers were identified by western blot. Activation of Caspase 3 (cleaved Caspase 3) and cleavage of PARP imply apoptosis induction after chemotherapeutic treatment. These apoptotic mediators were detectable for $24^{+} \mathrm{HMLE}$ as well as for $24^{+}$RAS cells, but not for MSP or MSP RAS cells (Figure 5.2B, C). Even higher concentrations of Cisplatin $(60 \mu \mathrm{M})$ did not result in generic detached rounded cells in case of MSP cells (Figure 5.2A). As an alternative measure to define apoptotic cells, activated caspases were determined by flow cytometry. MSP RAS cells treated with $30 \mu \mathrm{M}$ Cisplatin displayed less amounts of apoptotic cells compared to $24^{+}$RAS cells (Figure 5.2D), indicating lack of activation of apoptotic mediators in MSP and MSP RAS cells after Cisplatin treatment.

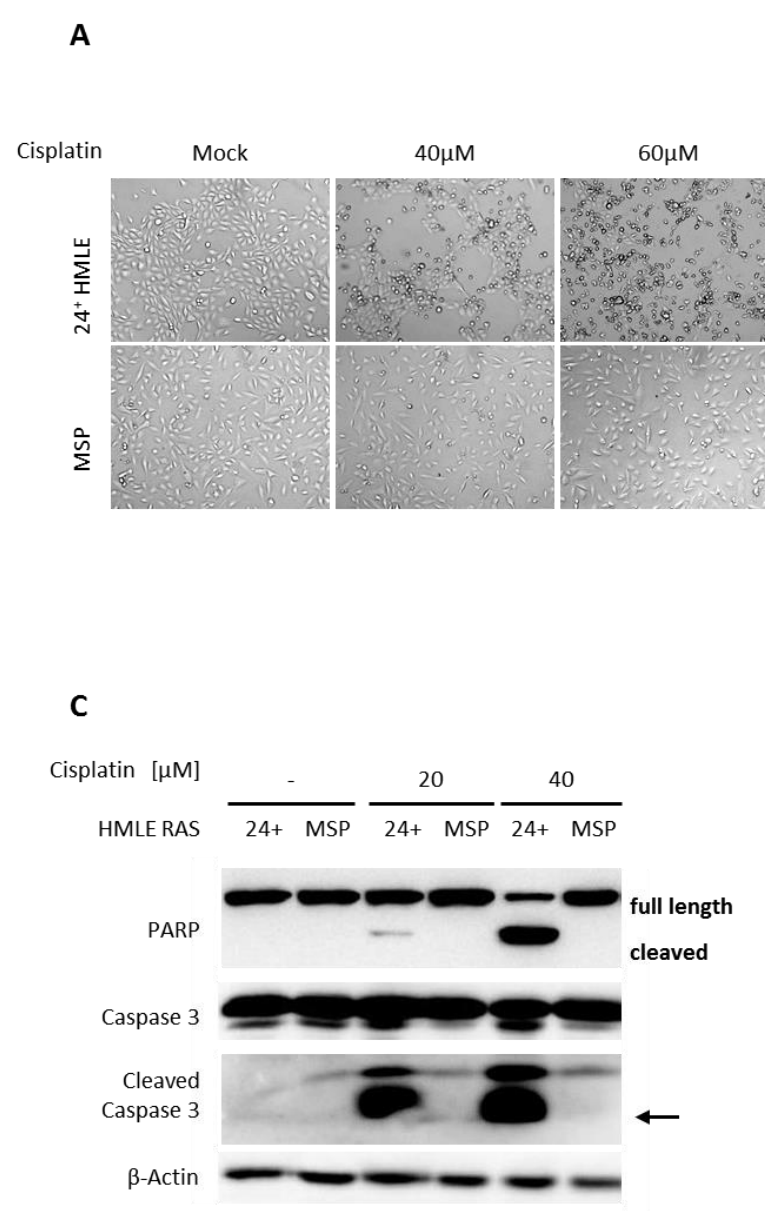

B

Cisplatin $[\mu \mathrm{M}]$
HMLE $\frac{-}{24+\mathrm{MSP}} \frac{20}{24+\mathrm{MSP}} \frac{40}{24+\mathrm{MSP}}$

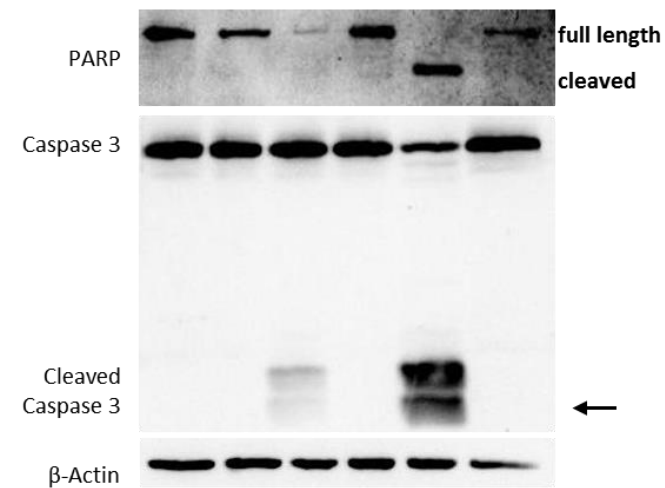

D

Multicaspase Activation

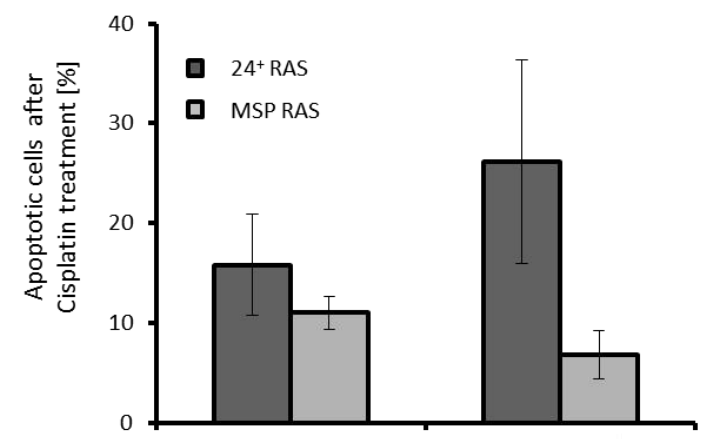

Cisplatin $[30 \mu \mathrm{M}]$

Figure 5.2: In response to Cisplatin no induction of apoptosis is detectable in MSP RAS cells.

(A) $16 \mathrm{~h}$ after seeding HMLE cells were treated with $40 \mu \mathrm{M}$ or $60 \mu \mathrm{M}$ Cisplatin for $8 \mathrm{~h}$. Thereafter, morphology of cells was checked with phase-contrast microscopy. (B, C) HMLE (B) and HMLE RAS cells (C) were treated with $20 \mu \mathrm{M}$ or $40 \mu \mathrm{M}$ Cisplatin for $16 \mathrm{~h}$. Cells were harvested and lysates were analyzed by western blot. Actin 
staining was used as loading control and arrows are indicating the intended staining. One representative experiment of $\mathrm{n}=3$ is shown. (D) $16 \mathrm{~h}$ after seeding HMLE RAS cells were treated with $30 \mu \mathrm{M}$ Cisplatin for $16 \mathrm{~h}$. Cells and supernatants were harvested and apoptotic cells were determined using the Guava® Multi Caspase FAM Kit and flow cytometry. Data show mean $\pm \mathrm{SD}$ of $\mathrm{n}=3$.

It was recently published that EMT activators are able to directly manipulate the cell-cycle progression impairing proliferative activity [122; 123]. Diminished cell proliferation is assumed to protect from DNA damage and would result in delayed or missing induction of apoptosis upon chemotherapeutic treatment [124]. We therefore performed cell proliferation assays in HMLE as well as HMLE RAS cells. Proliferation was measured over $2 \mathrm{~d}$ by direct cell counting, defining the cell number per well using a Celigo ${ }^{\circledR}$ cytometer. The analysis showed that $24^{+}$HMLE cells displayed different growth rates compared to MSP cells, while they were comparable for HMLE RAS cells (Figure 5.3A, B mock). Implicating that different growth rates are not the reason for lacking apoptosis, we decided to use the HMLE RAS cell system for further analysis. To this end, HMLE RAS cells were treated with $5 \mu \mathrm{M}$ or $10 \mu \mathrm{M}$ Cisplatin for $24 \mathrm{~h}$ and subsequently, Cisplatin was replaced by fresh medium and the cell proliferation were measured over $7 \mathrm{~d}$ every $24 \mathrm{~h}$ by direct cell counting. Strikingly, MSP RAS cells continued their proliferation $5 \mathrm{~d}$ after Cisplatin withdrawal, while $24^{+}$RAS cells did not recover (Figure 5.3B). Considering these results, we were interested to know whether Cisplatin is absorbed by MSP RAS and explored the Cisplatin adducts that were formed between the platinum atom and DNA after Cisplatin treatment. We made use of an Immuno-Slot-Blot assay in cooperation with J. Thomale, University of Duisburg-Essen, applying an antibody that specifically recognizes the platinum atom bound to $\mathrm{GpG}$ [14]. These data demonstrated that $24^{+}$RAS and MSP RAS display equal amounts of Cisplatin adducts after treatment (Figure 5.4). This experiment argues that DNA of $24^{+}$RAS cells and MSP RAS cells is damaged to the same degree by chemotherapy. Thus, neither different cell proliferation nor Cisplatin transport processes are involved in apoptosis resistance of MSP RAS cells. 


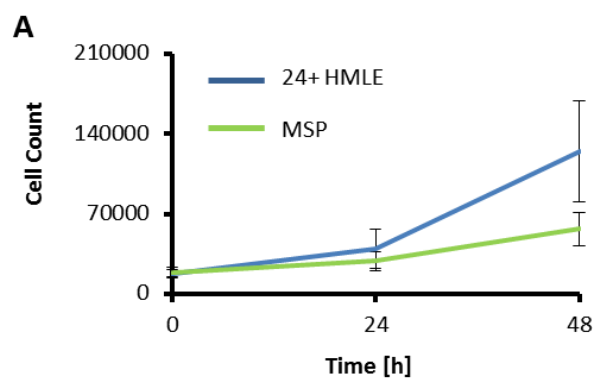

B
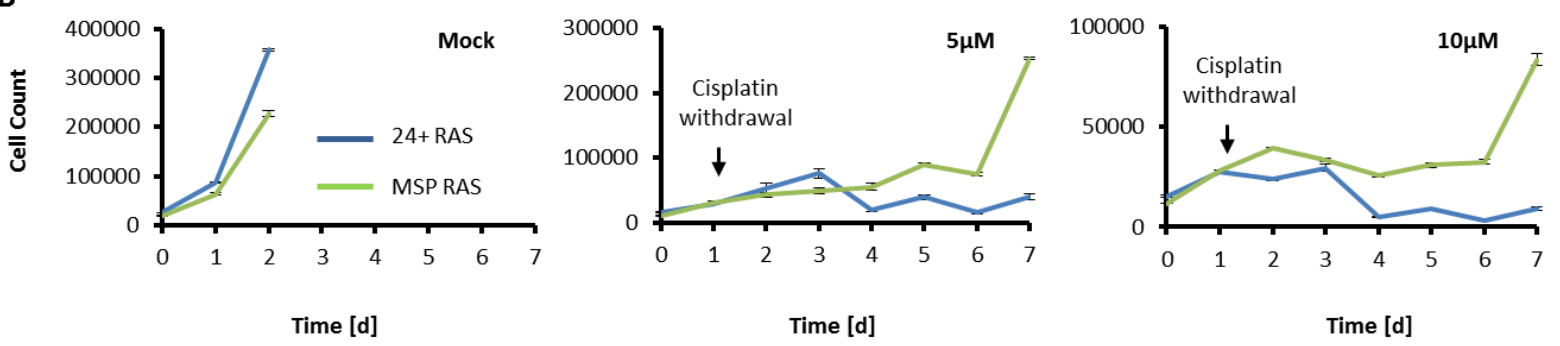

Figure 5.3: MSP RAS cells recover from Cisplatin treatment.

(A) $8 \mathrm{~h}$ after seeding HMLE cells, direct cell counting was performed for $48 \mathrm{~h}$ by defining the cell number per well using a Celigo $®$ cytometer. One representative experiment of $n=3$ is shown. (B) $24 \mathrm{~h}$ after seeding HMLE RAS were treated with $5 \mu \mathrm{M}$ or $10 \mu \mathrm{M}$ Cisplatin for $24 \mathrm{~h}$. Afterwards, cells were washed with supplemented MEGM medium and kept in culture. Cell number was measured once a day for $7 \mathrm{~d}$ with a Celigo® cytometer after adding fresh medium. Non-treated cells were used as control. Data show mean $\pm \mathrm{SD}$. One representative experiment of $n=3$ is shown.

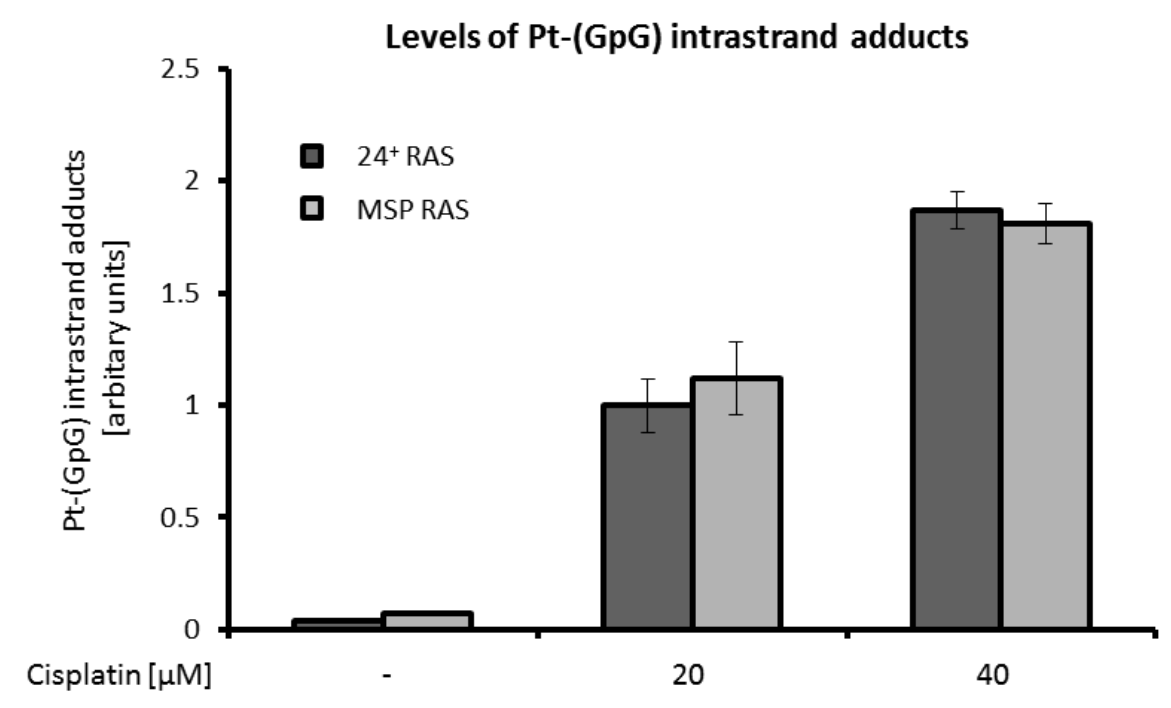

Figure 5.4: Cells before and upon EMT display equal amounts of Cisplatin adducts.

24h after seeding HMLE RAS cells were treated with $20 \mu \mathrm{M}$ or $40 \mu \mathrm{M}$ Cisplatin for $16 \mathrm{~h}$. Afterwards, DNA was extracted with NucleoBond $\AA$ AXG columns and analyzed for formed adducts by Immuno-Slot-Blot assay. Experiments were conducted with J. Thomale, University of Duisburg-Essen. Non-treated cells were used as control. Data show mean $\pm \mathrm{SD}$ of $\mathrm{n}=3$. 


\subsubsection{MSP RAS cells display a general apoptosis deficit}

Given the above results, we made use of different inhibitors manipulating distinct pathways that are involved in EMT or cell survival to test whether this would sensitize MSP RAS cells to Cisplatin treatment: First, we inhibited the Akt-signalling pathway by Akt and mTor inhibitors. Akt is a pro-survival protein, which is described as mediator of chemoresistance to Cisplatin [125]. Furthermore, we manipulated the mitogen activated protein kinase Mek and inhibited JNK and p38, which are involved in the activated Wnt-signalling pathway [126]. MSP RAS cells were treated with the respective inhibitors in presence or absence of Cisplatin; subsequently, cells were harvested and analyzed for induced apoptosis mediators by western blot. None of the treatments resulted in PARP cleavage; implicating no effects on the response to Cisplatin in MSP RAS cells (Figure 5.5A, C, D). Even by inhibiting TGF $\beta$-induced pathways no changes concerning cleaved PARP were detectable for MSP RAS cells (Figure 5.5B). On that account, it was tested whether other chemotherapeutics were able to induce apoptosis in MSP RAS cells. To this end, HMLE RAS cells were exposed to Carboplatin, Doxorubicin or Neocarzinostatin (NCS) followed by western blot analyses for PARP cleavage. Strikingly, none of the chemotherapeutics induced apoptosis in MSP RAS cells while $24^{+}$RAS cells showed PARP cleavage for every treatment (Figure 5.6). These findings raised the question whether it is possible to induce apoptosis in MSP RAS cells independent from DNA damage. To test this, HMLE RAS cells were exposed to several concentrations of the death ligands Trail or TNFa. Since these ligands induce the transcription of NFאB and thus pro-survival pathways as a negative feedback, all treatments were performed in the presence of Cycloheximide a protein synthesis inhibitor preventing NFKB translation [127]. For $24^{+}$RAS cells activation of Caspase 3 as well as PARP cleavage was detectable for both treatments. But even for higher concentrations of Trail no PARP cleavage was identified for MSP RAS cells (Figure 5.7A). Only for TNF $\alpha$ concentrations four times higher compared to the concentrations used for $24^{+}$RAS cells, activation of the apoptosis mediators was observed (Figure 5.7B).

In summary, these results suggest that EMT results in MSP RAS cells that are resistant to the induction of apoptosis through chemotherapy or independent from DNA damage through death ligands. 


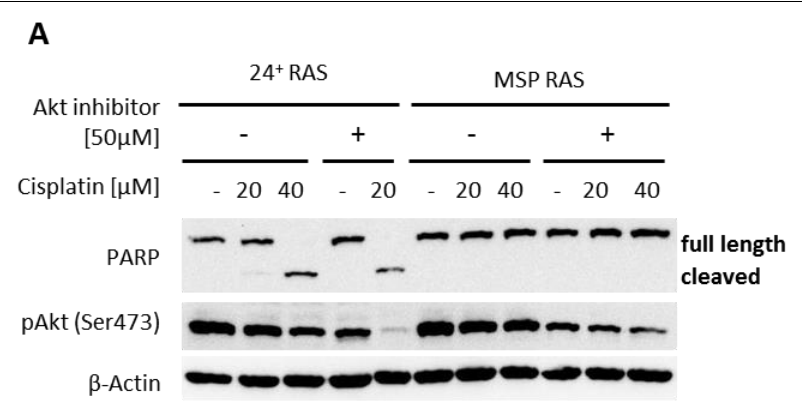

C

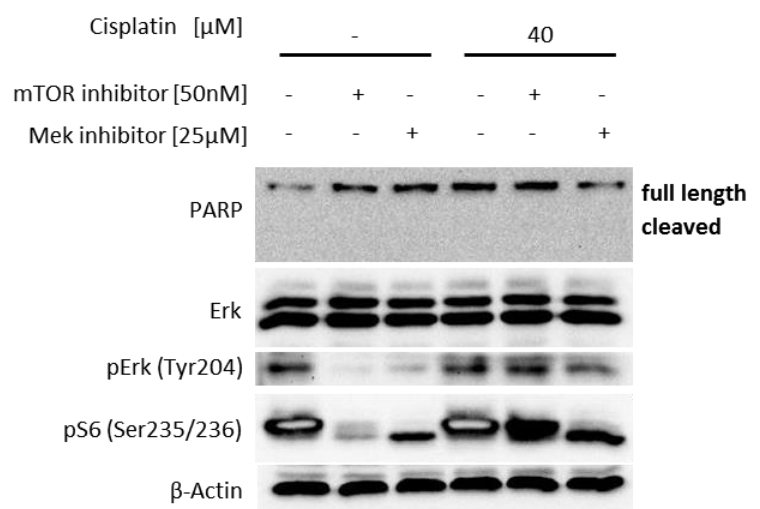

B

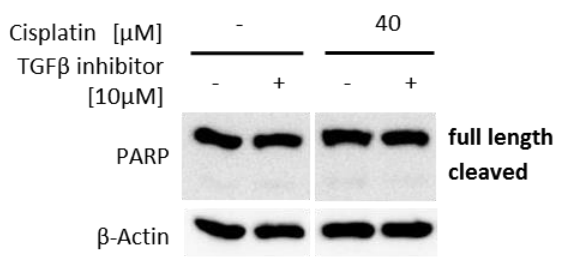

D

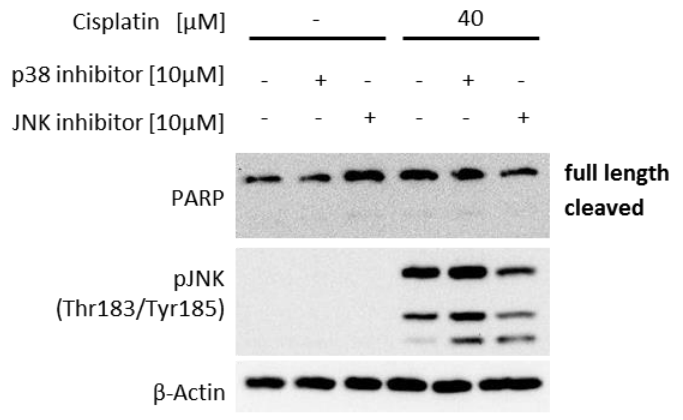

Figure 5.5: Manipulation of distinct signalling pathways does not sensitize MSP RAS cells towards Cisplatin.

(A-D) 24h after seeding HMLE RAS cells (A) or MSP RAS cells alone (B-D) were treated with the indicated inhibitors of different signalling pathways in the presence or absence of $20 \mu \mathrm{M}$ or $40 \mu \mathrm{M}$ Cisplatin for $16 \mathrm{~h}$. Afterwards, cells were harvested and lysates were analyzed by western blot. Actin staining was used as loading control. Controls were treated with similar amounts of DMSO, $\mathrm{n}=1$.

A

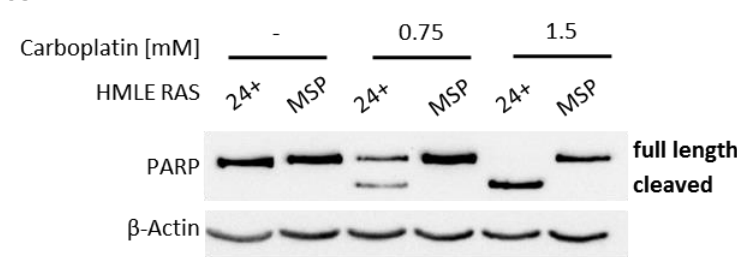

C

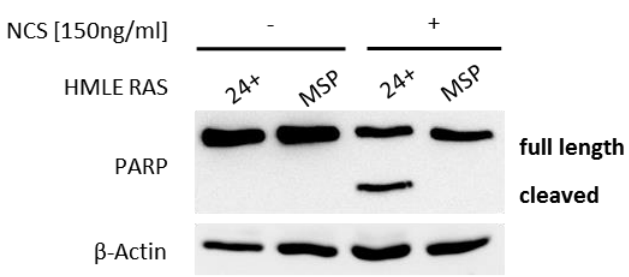

B

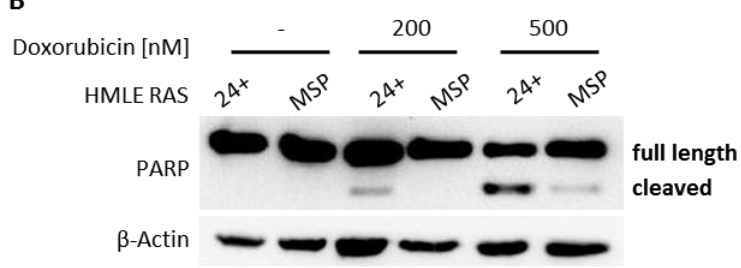

Figure 5.6: MSP RAS are resistant to a variety of chemotherapeutics.

(A-C) 16h after seeding HMLE RAS cells were treated with the indicated concentrations of Carboplatin for $16 \mathrm{~h}$ (A), Doxorubicin for $24 \mathrm{~h}$ (B) or NCS for $20 \mathrm{~h}$ (C). Thereafter, cells were harvested and lysates were analyzed by western blot. Actin staining was used as loading control. Controls were treated with similar amounts of the respective solvent, $\mathrm{n}=1$. 

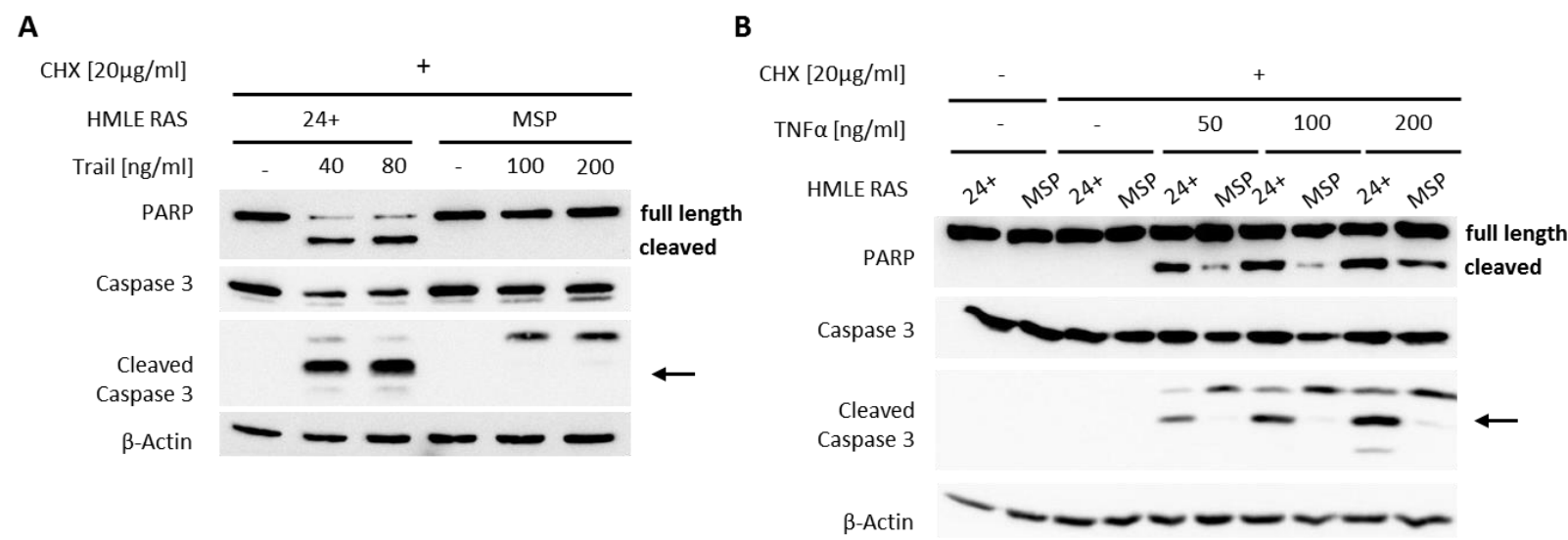

Figure 5.7: In response to the treatment with death ligands no apoptosis is detectable in MSP RAS cells.

(A, B) 16h after seeding HMLE RAS cells were treated with the indicated concentrations of Trail (A) or TNF $\alpha$ (B) in presence of $20 \mu \mathrm{g} / \mathrm{ml} \mathrm{CHX}$ for $6 \mathrm{~h}$. Subsequently, cells were harvested and lysates were analyzed by western blot. Controls were treated with Ethanol for CHX or sterile $\mathrm{H}_{2} \mathrm{O}$ for Trail and TNF $\alpha$. Actin staining was used as loading control and arrows are indicating the intended staining. Shown is one representative experiment of $\mathrm{n}=3$.

\subsection{Bcl-xL overexpression protects MSP RAS cells from chemotherapy}

\subsubsection{EMT causes overexpression of the anti-apoptotic protein $\mathrm{Bcl}-\mathrm{xL}$}

Previous results indicated that the failure of induction of apoptosis in MSP RAS cells is independent of chemotherapeutic treatment or exposure to death ligands. We hypothesized that mediators related to apoptosis are changed in cells upon EMT. We therefore checked our micro array data for differentially regulated genes that are associated with apoptosis. In fact, gene expression of the apoptotic gene Bcl2L1 was found to be two fold up-regulated in MSP cells compared to $24^{+}$HMLE cells (appendix). The Bc12L1 gene encodes two different proteins: the pro-apoptotic variant Bcl-xS and the anti-apoptotic variant Bcl-xL [128]. Quantitative RT-PCR for HMLE and HMLE RAS cells showed a two to three fold induction of Bcl2L1, Bcl-xS and Bcl-xL in MSP and MSP RAS cells compared to $24^{+} \mathrm{HMLE}$ and $24^{+} \mathrm{RAS}$ cells, respectively. We further checked the protein levels for Bcl2L1 by western blot and found that $\mathrm{Bcl} 2 \mathrm{~L} 1$ is highly overexpressed in MSP and MSP RAS cells compared to $24^{+}$HMLE and $24^{+}$RAS cells, respectively (Figure 5.8A, B). To assess whether the anti- or pro-apoptotic variant of Bcl2L1 is up-regulated in MSP and MSP RAS cells, we used an antibody that recognizes an epitope that is solely present in Bcl-xL, but missing in Bcl-xS. We obtained the same result for the specific (sc-8392) compared to the unspecific Bcl-xL antibody (cs-2764), i.e. only one signal at the height of Bcl-xL (26kDa) and no signal correspond- 
ing to Bcl-xS (19kDa) (Figure 5.8C). Apart from Bcl-xL, we were not able to detect other upregulated anti-apoptotic proteins belonging to the Bcl-2 family, such as Mcl-1 or Bcl-2 (Figure 5.8D).

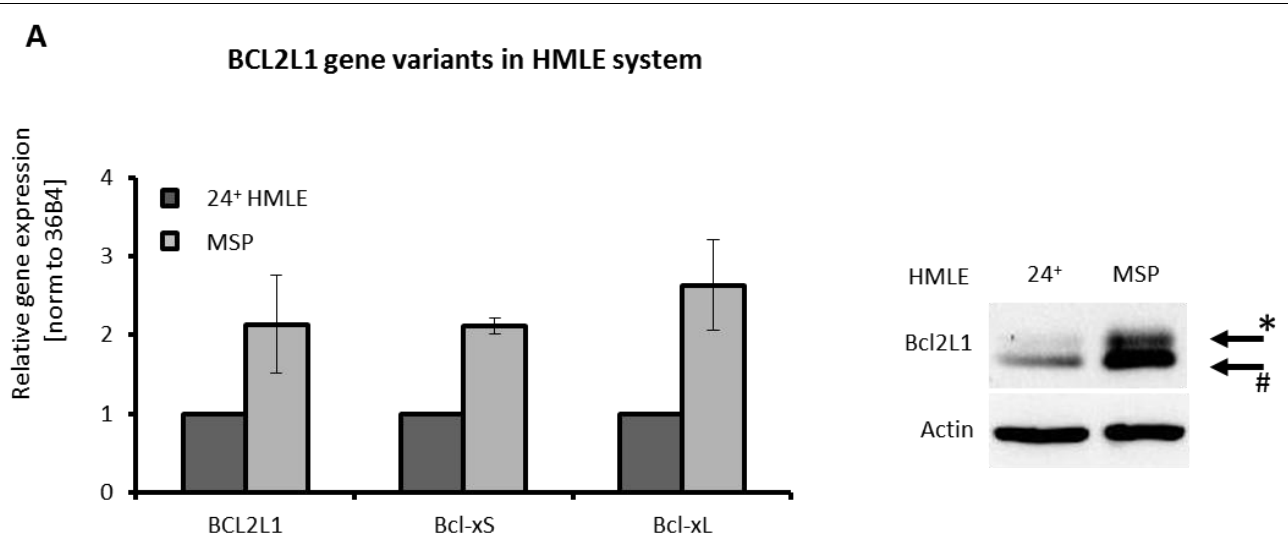

B

BCL2L1 gene variants in the HMLE RAS system
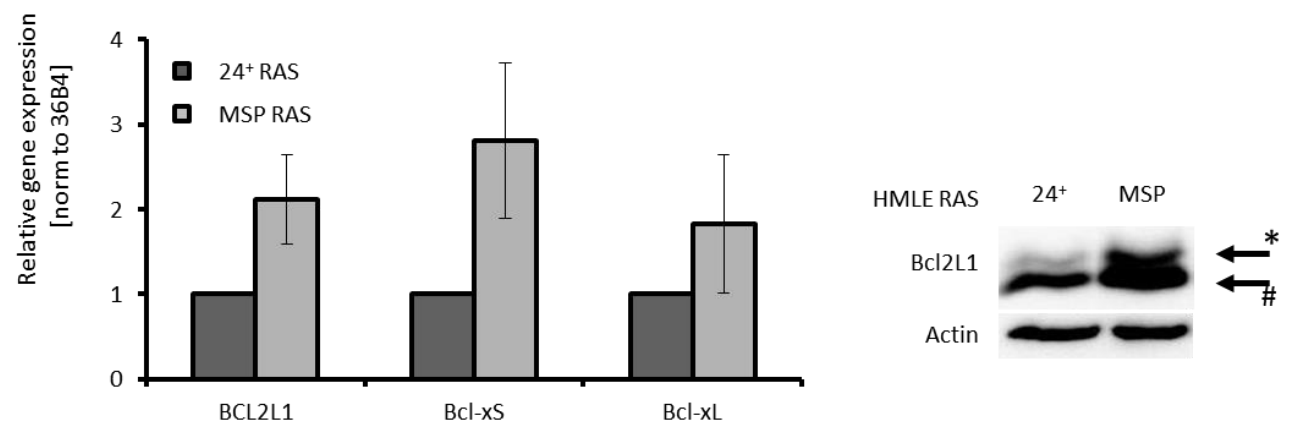

C

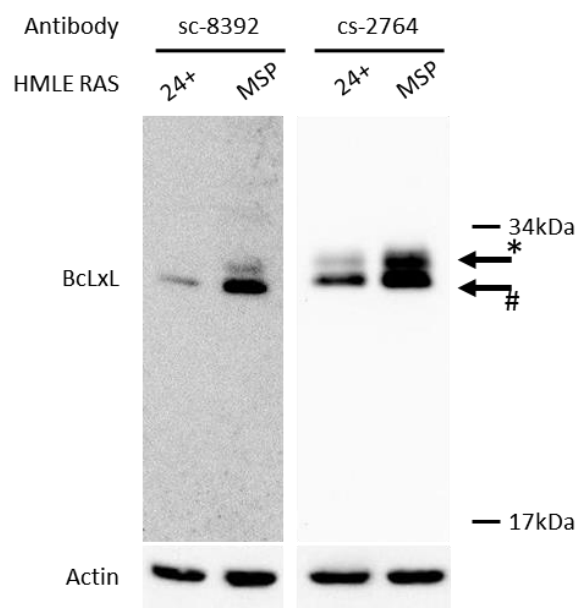

D

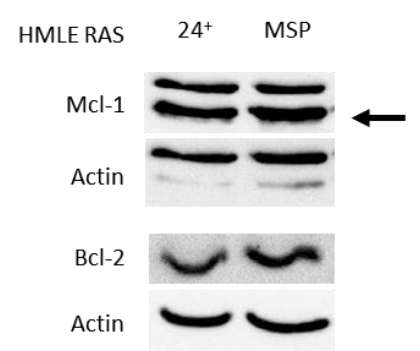

Figure 5.8: EMT results in overexpression of the anti-apoptotic protein Bcl-xL.

(A, B) HMLE (A) and HMLE RAS cells (B) were harvested 24h after seeding, mRNA was isolated and mRNA level of Bcl2L1 variants were analyzed using qRT-PCR. mRNA level were normalized to 36B4. Data show mean \pm SD of $n=3$. Protein lysates were analyzed for Bcl2L1 by western blotting. (C, D) HMLE RAS were harvested $24 \mathrm{~h}$ after seeding and lysates were analyzed for anti-apoptotic proteins by western blotting. Actin staining was used as loading control and arrows are indicating the intended staining. For Bcl2L1 and Bcl-xL: $\leftarrow_{*}^{*}$ indicates deamidated protein, $\leftarrow \#$ indicates unmodified protein. For western blot one representative experiment of $\mathrm{n}=3$ is shown. 


\subsubsection{Bcl-xL siRNA knock down sensitizes MSP RAS cells to apoptosis induction}

To investigate the role of $\mathrm{Bcl}-\mathrm{xL}$ in chemotherapeutic resistance, we performed $\mathrm{Bcl}-\mathrm{xL}$ mediated siRNA knock down, treated the cells with Cisplatin and subsequently, analyzed cell lysates for apoptosis induction by western blot. siRNA targeting Bcl-xL efficiently depleted cells of the protein (Figure 5.9A). Compared to mock and scr control-treated cells, Bcl-xLdepleted MSP RAS cells displayed strong activation of Caspase 3 as well as PARP cleavage in the presence of Cisplatin (Figure 5.9A). Analyzing the activated caspases with flow cytometry for Bcl-xL-depleted MSP RAS cells a significant increase in the amount of apoptotic cells was visible (Figure 5.9B). This confirmed the results obtained with western blot. Furthermore, we found that the Bcl-xL-mediated siRNA knock down caused apoptosis in MSP RAS cells after exposure to Trail, independent from DNA damage (Figure 5.9C). Thereupon, it was tested whether overexpression of Bcl-xL would rescue the $24^{+} \mathrm{HMLE}$ from induction of apoptosis after Cisplatin or Trail treatment. For this purpose, Weinberg and colleagues provided us with $24^{+} \mathrm{HMLE}$ cells that stably overexpress Bcl2L1 $\left(24^{+\mathrm{Bcl}-\mathrm{xL}}\right)$ and applied Cisplatin and Trail to these cells. Indeed, $24^{+\mathrm{Bcl}-\mathrm{xL}}$ displayed neither activated Caspase 3 nor cleaved PARP (Figure 5.10A, B). These results support the notion of Bcl-xL as a key mediator in preventing apoptosis in cells upon EMT and implicates that the failure of apoptosis induction in MSP RAS cells is not caused downstream of Bcl-xL.

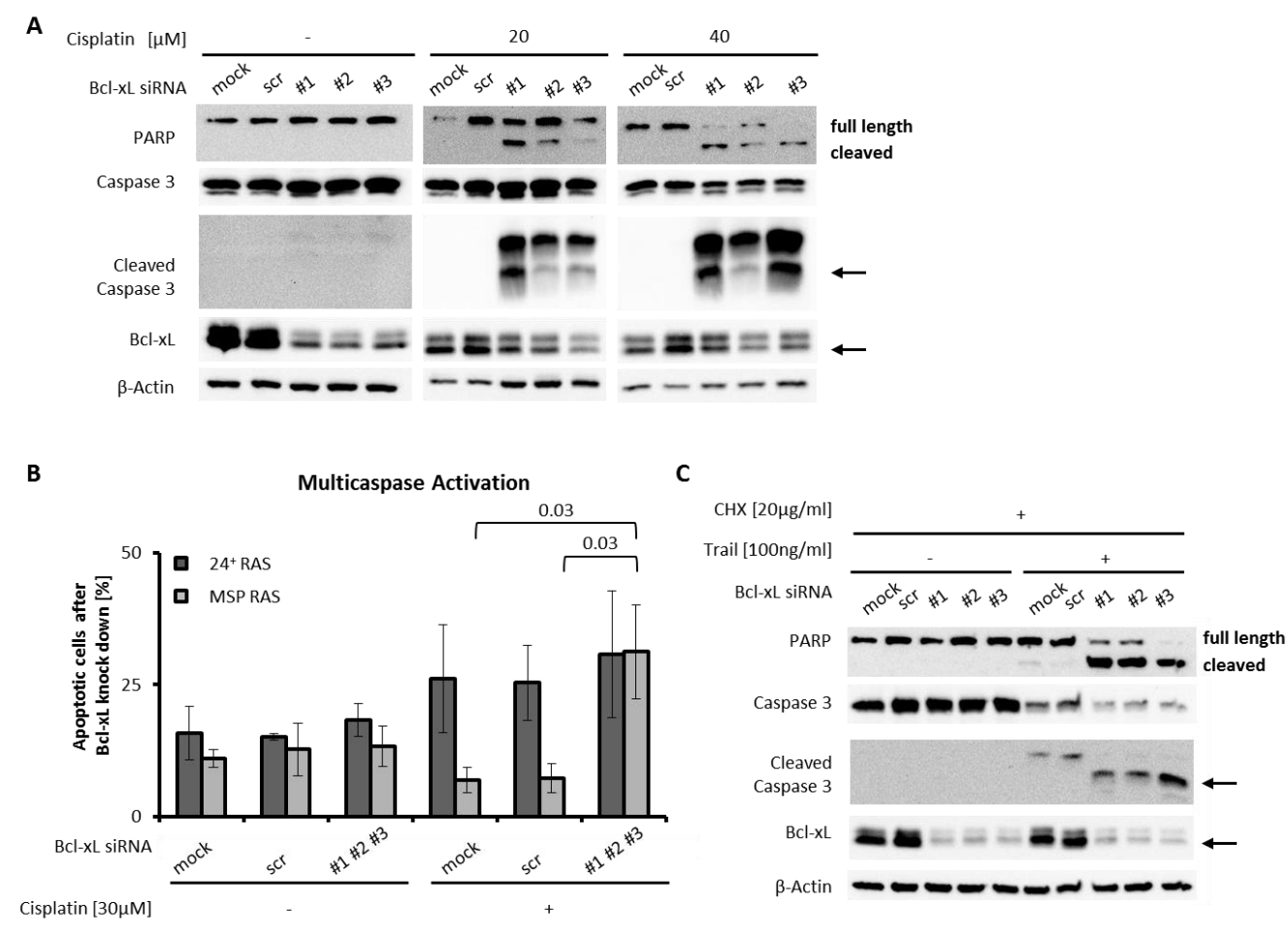

Figure 5.9: Bcl-xL siRNA knock down sensitizes MSP RAS cells to Cisplatin and Trail treatment. Legend on the next page. 
Figure 5.9: Bcl-xL siRNA knock down sensitizes MSP RAS cells to Cisplatin and Trail treatment. (A) $32 \mathrm{~h}$ after siRNA mediated knock down of Bcl-xL, MSP RAS cells were treated with $20 \mu \mathrm{M}$ or $40 \mu \mathrm{M}$ Cisplatin for 16h. Subsequently, cells were harvested and lysates were analyzed by western blot. (B) $32 \mathrm{~h}$ after siRNA mediat-

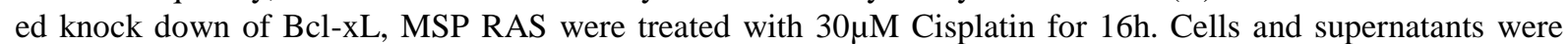
harvested and apoptotic cells were determined using the Guava ${ }^{\circledR}$ Multi Caspase FAM Kit and flow cytometry. Data show mean \pm SD of $n=3$. Student's t-test was performed for statistical analyses. (C) $48 \mathrm{~h}$ post transfection MSP RAS cells were treated with $100 \mathrm{ng} / \mathrm{ml}$ Trail in the presence of $20 \mu \mathrm{g} / \mathrm{ml} \mathrm{CHX}$ for $6 \mathrm{~h}$. Thereafter, cells were harvested and lysates were analyzed by western blot. Untreated and scr siRNA-transfected cells were used as control, while Actin staining was used as loading control. Arrows are indicating the intended staining. A, C: One representative experiment of $n=3$ is shown.

A

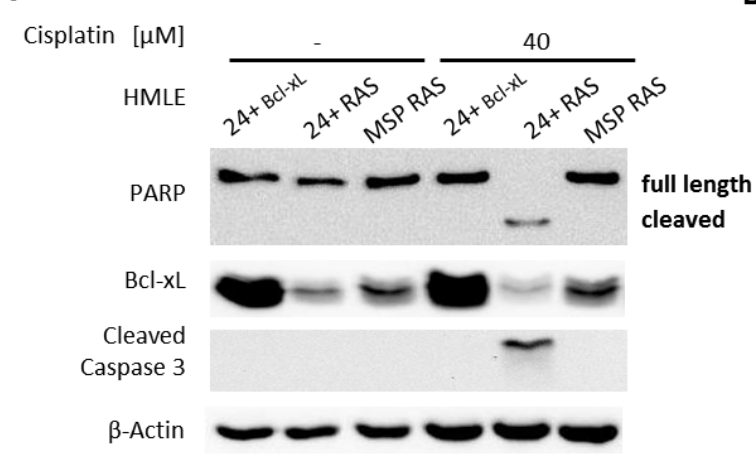

B

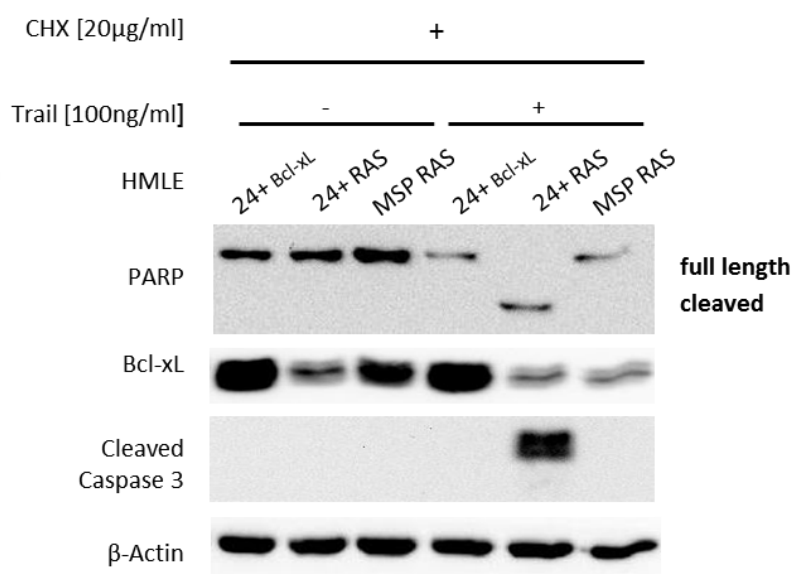

Figure 5.10: Bcl-xL overexpression in 24+HMLE cells results in resistant cells.

(A, B) $24^{+B c l-x L}$ and HMLE RAS cells were treated with $40 \mu \mathrm{M}$ Cisplatin for $16 \mathrm{~h}$ (A) or 100ng/ml Trail in the presence of $20 \mu \mathrm{g} / \mathrm{ml} \mathrm{CHX} \mathrm{for} 6 \mathrm{~h}$ (B). Subsequently, cells were harvested and lysates were analyzed by western blot. Untreated cells were used as control, while Actin staining was used as loading control. $n=1$.

\subsubsection{Bcl-xL protein level are increased in disseminated tumor cells in human tissue}

Additionally, we were interested to see whether we can transfer our results to clinical breast cancer tissues. We therefore collaborated with A. Scheel, Institute for Pathology Nordhessen, and assessed Bcl-xL protein level in human ductal carcinoma (DIC). We made use of immunohistochemistry on formalin-fixed paraffin-embedded tumor material. 26 cases of DIC including all molecular subtypes of human breast cancer as luminal A, B and triple negative, were validated for Bcl-xL level. The data showed that Bcl-xL was detected in every specimen but differences in protein levels did not correlate with subtypes or the parameters of hormone receptors, Her2 status, stage, age and sex. Bcl-xL protein expression was heterogeneously distributed within the respective specimen, whereby six cases display pronounced protein levels in tumor cells at the tumor-stroma-interface and in disseminated tumor cells (Figure 5.11A). As an alternative measure, digital color intensity quantification corroborated the increased Bcl-xL staining (Figure 5.11B). The disseminated high positive Bcl-xL cells expressed the epithelial marker Cytokeratin 8, indicating that morphologically these cells seem to originate 
from the solid tumor formations (Figure 5.11C). However, the observed heterogeneous expression of $\mathrm{N}$-Cadherin that partly overlapped with Bcl-xL expression implies that these are altered cells from the solid tumor [personal communication A. Scheel] (Figure 5.11D).

A

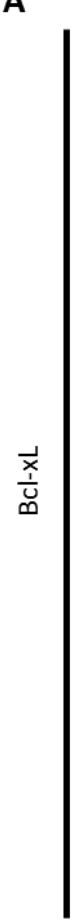

Image

Case \#1 (H67889-10)

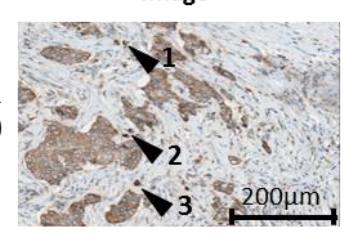

(H10708-11)

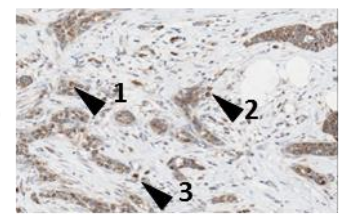

(H29296-10)

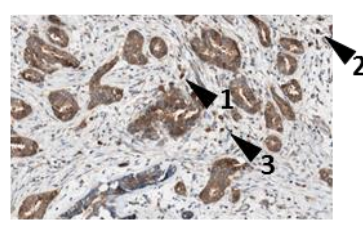

\#4

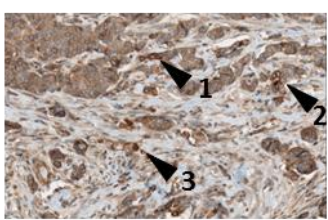

Image, details
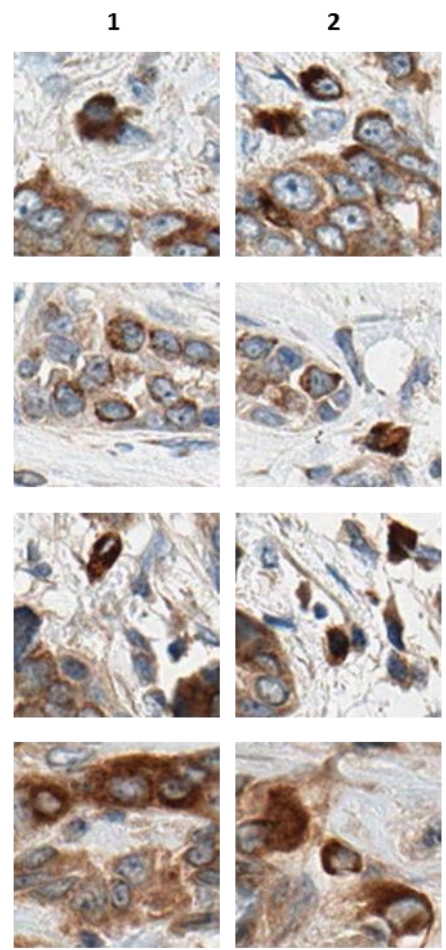
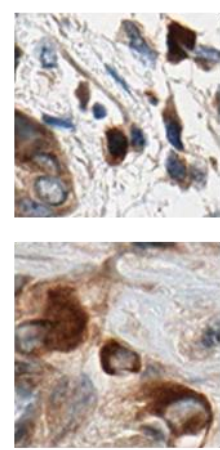

3
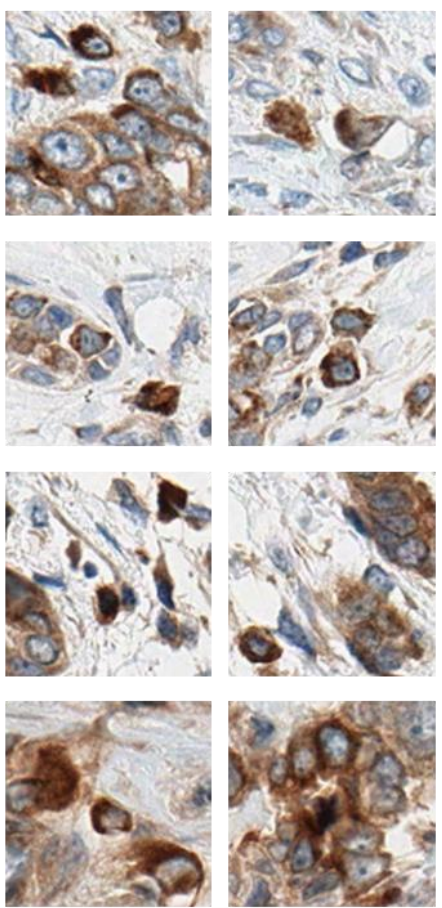

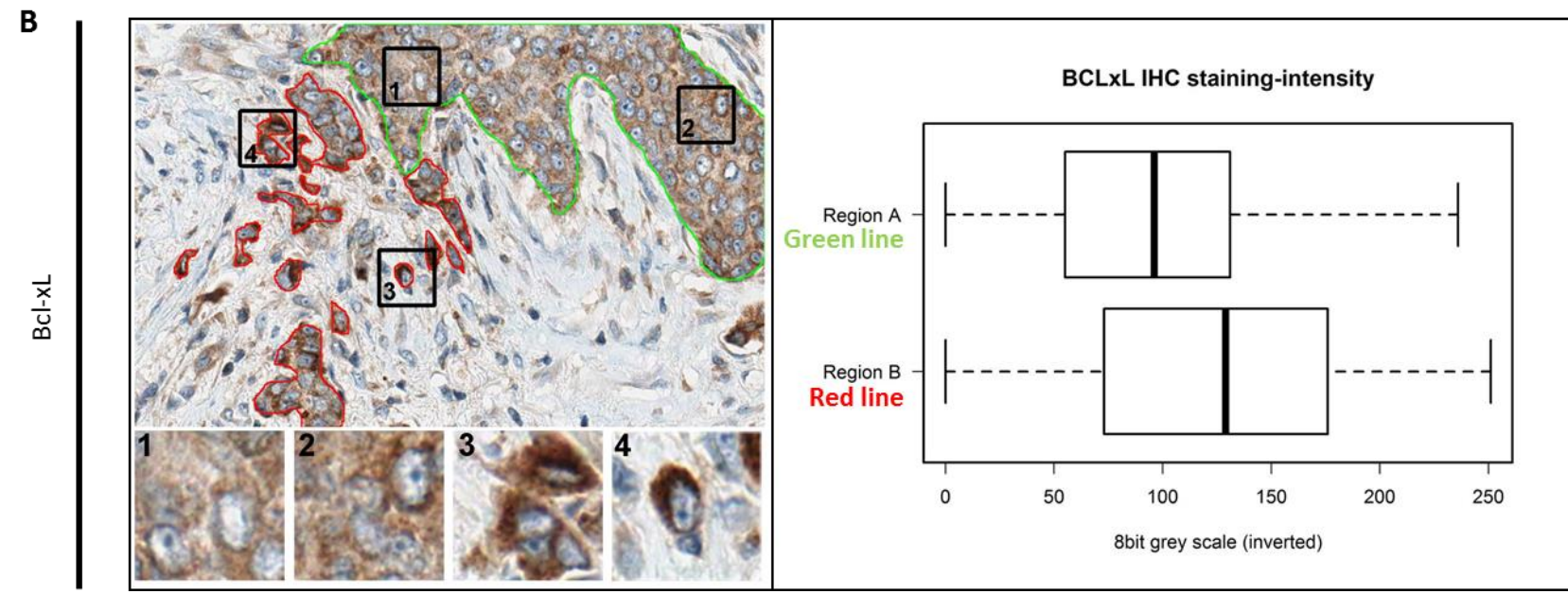

Figure 5.11: Bcl-xL protein levels are increased at the tumor-stroma-interface and in disseminated tumor cells.

Continued on the next page. 
C

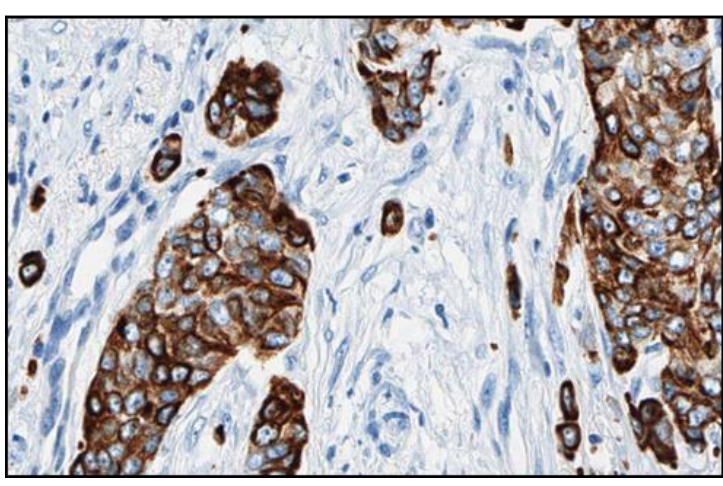

D

$\mathrm{N}$-Cadherin

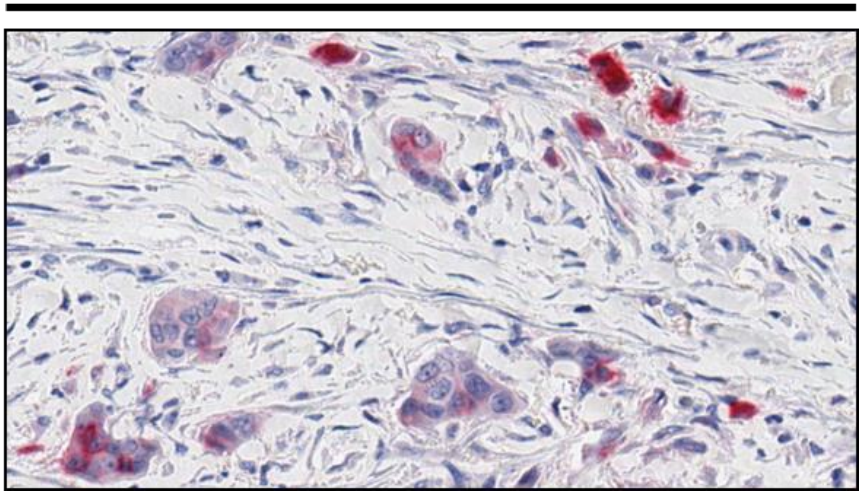

Figure 5.11: Bcl-xL protein levels are increased at the tumor-stroma-interface and in disseminated tumor cells. (A-D) Human tissue fixed in PBS-buffered $4 \%$ formalin was embedded in paraffin. $1.5 \mu \mathrm{m}$ sections were cut and treated with boric-acid/EDTA buffer for antigen-retrieval (A) IHC for Bcl-xL. 3 images of 4 clinical cases, magnification 200x. Column 1: Representative cells highlighted (black arrows), columns 2-4: Magnifications of areas indicated in the first column. (B) Quantification of DAB precipitate color-intensity in the two highlighted regions (borders green, red); boxplots of respective intensities. '1'-'4': Magnified details as indicated. (C) Cytokeratin 8 IHC. (D) N-Cadherin IHC (Fast Red). B-D: 400x magnification, corresponding regions. Experiments were conducted with A. Scheel, Institute for Pathology Nordhessen.

\subsubsection{Bcl-xL level in MSP RAS cells are not detectably regulated by translation, tran- scription factors, miRNA or proteasomal degradation}

Since we have shown that EMT obviously causes the overexpression of the anti-apoptotic protein Bcl-xL in MSP and MSP RAS cells, we were interested to examine how this induction is regulated. To assess, whether translational or proteasomal processes are involved we made use of time chase experiments and exposed the HMLE RAS cells to Cycloheximide or MG132 up to 4h and analyzed protein lysates for Bcl-xL afterwards. Cycloheximide inhibits the elongation of translation by binding the ribosome and inhibiting eEF2-mediated translocation of the mRNA [129]. Regulation on this level would result in decreased protein levels as it is visible for the control staining Hif1 $\alpha$. Bcl-xL protein levels were neither affected by Cycloheximide in 24+ RAS cells nor in MSP RAS cells (Figure 5.12A). In contrast to Cycloheximide, the proteasomal inhibition by MG132 would result in protein accumulation. This was the case for the control staining Hif $1 \alpha$, but no increase were observed for the levels of Bcl-xL in HMLE RAS (Figure 5.12B), suggesting that Bcl-xL is not a high-turnover protein.

It was recently published that miRNAs (miR) are directly associated with EMT in development and cancer. Peter reviewed that especially let7 miRNA / miR-98 and the miR-200 family are linked to cell differentiation and EMT [130]. Additionally, Shimizu et al. showed that low let7 miRNA expression results in increased Bcl-xL protein levels [79]. To investigate the role of miRNA concerning the overexpression of Bcl-xL in MSP RAS cells we used Taq- 
man ${ }^{\circledR}$ PCR as an alternative measure to define the expression levels of miRNA let7a, $b$ and let7f in HMLE RAS cells. However, quantification of miRNA let7 revealed that the expression levels do not differ in HMLE RAS cells (Figure 5.13A). Apart from miRNA let7, the miR-200 family is involved in processes that regulate EMT. In this context, miR-200 expression contributes to an epithelial phenotype by preventing the expression of the transcriptional repressors Zeb1 and Zeb2, which are highly induced upon EMT [131]. To explore, whether miRNAs are involved in Bcl-xL translation in general, we decided to perform a siRNAmediated knock down of Dicer, a protein that is necessary for the maturation of miRNA from pre-miRNA. Depletion of Dicer abrogates the processing of pre-miRNA and therefore depletes the cell of mature miRNAs. If Bcl-xL mRNA is degraded by miRNA, it would be expected that upon Dicer siRNA knock down Bcl-xL level are increased in $24^{+}$RAS cells. The knock down efficiency was monitored by qRT-PCR, but we did not detect any changes in Bcl-xL level for $24^{+}$RAS cells or MSP RAS cells (Figure 5.13B, C).

\section{A}

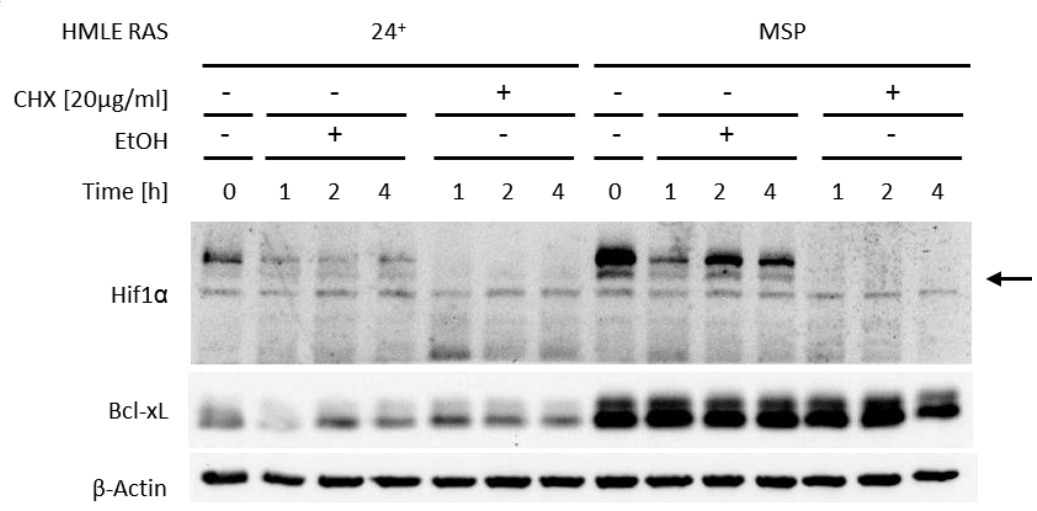

B

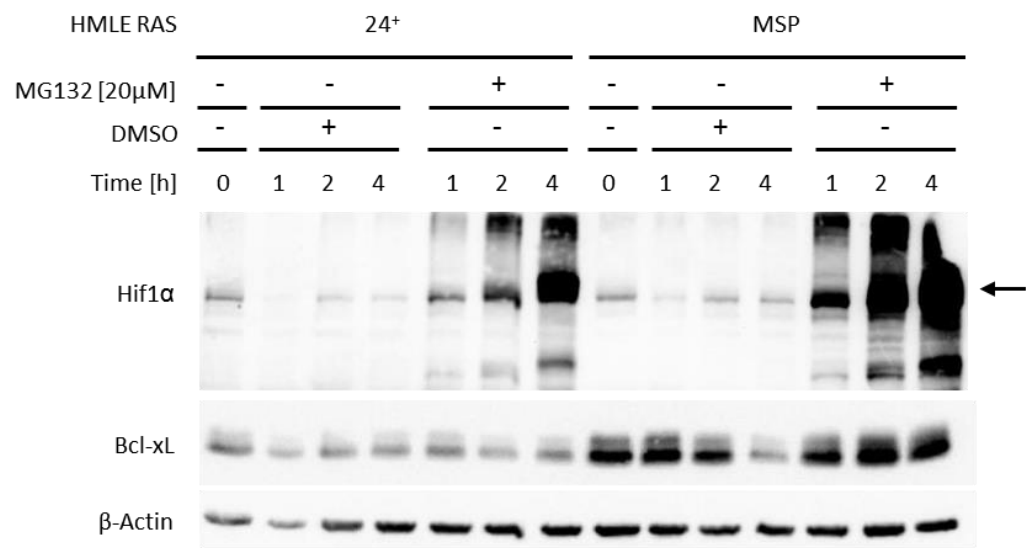

Figure 5.12: Bcl-xL overexpression is not detectably regulated by translation or proteasomal degradation. Legend on the next page. 
Figure 5.12: Bcl-xL overexpression is not detectably regulated by translation or proteasomal degradation.

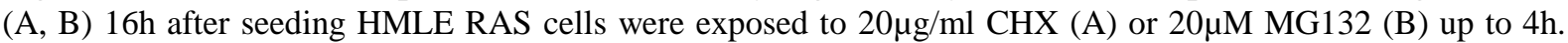
Thereafter, cells were harvested and lysates were analyzed for Bcl-xL by western blot. Actin staining was used as loading control, while Hif1 $\alpha$ staining was used as positive control for treatment. Ethanol or DMSO was used as control for CHX (A) or MG132 (B) treatment, respectively. Arrows are indicating the intended staining. $\mathrm{n}=1$.

A

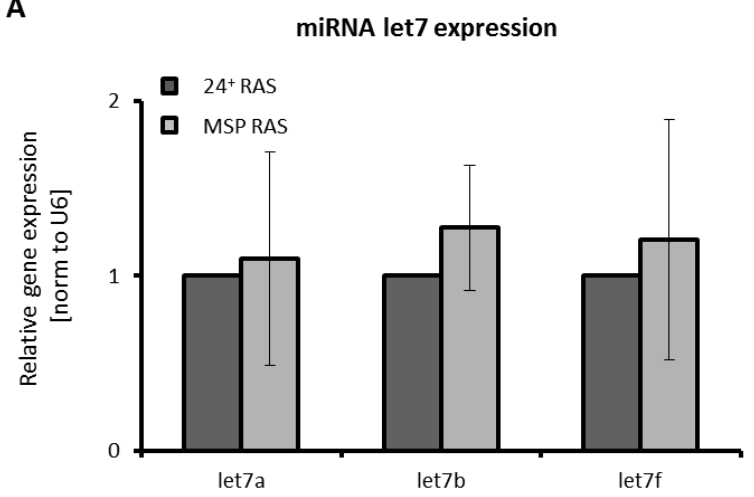

B

Dicer gene expression after Dicer siRNA knock down

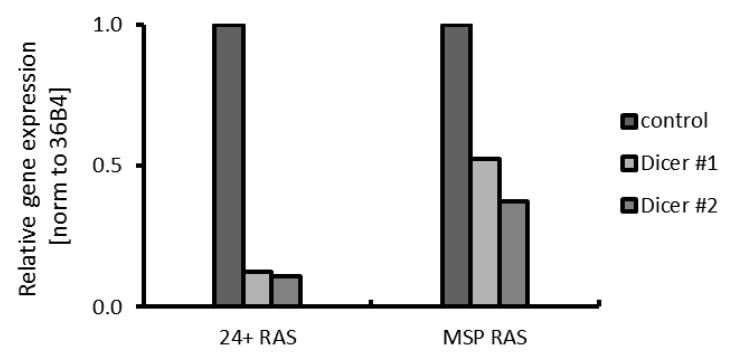

C

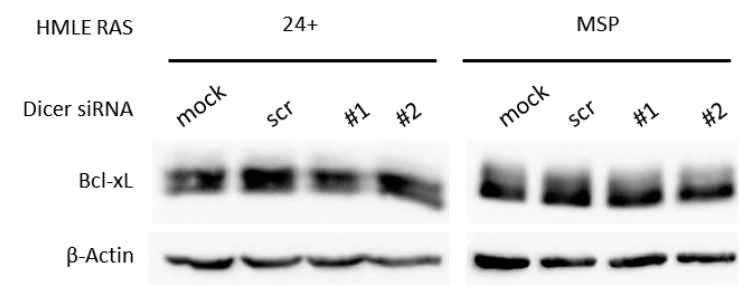

Figure 5.13: Bcl-xL overexpression is not detectably regulated by miRNAs.

(A) HMLE RAS cells were harvested 24h after seeding. Total mRNA was isolated and miRNA levels of miRNA let7 variants were analyzed using Taqman ${ }^{\circledR}$ PCR. miRNA levels were normalized to U6. Data show mean \pm SD of $n=3$. (B) $48 \mathrm{~h}$ after siRNA-mediated Dicer knock down HMLE RAS cells were harvested. mRNA was isolated and mRNA levels of Dicer were analyzed using qRT-PCR. mRNA levels were normalized to 36B4. Nontransfected cells or scr siRNA were used as control. $n=1$ (C) $48 \mathrm{~h}$ after siRNA-mediated Dicer knock down HMLE RAS cells were harvested and lysates were analyzed for Bcl-xL by western blot. Actin staining was used loading control. $\mathrm{n}=1$.

Scheel et al. have shown that the Wnt-signalling pathway is activated in MSP cells [110]. Active Wnt pathway results in phosphorylated JNK and subsequently in activated cJun. The transcriptional activator cJun is a pivotal component of the AP-1 transcription factor complex and able to induce autonomous gene expression [132]. In general, the dimeric transcription factor AP-1 consists of proteins that belong to the Jun (e.g. cJun and JunD), Fos (e.g. cFos), Maf and ATF family and bind to the AP-1 binding site [74]. The promoter region of Bcl-xL 
contains various transcription factor binding sites including two sites for AP-1 [133]. We therefore addressed the question, whether manipulation of AP-1 would alter Bcl-xL level in MSP RAS cells. Initially, we were able to validate the cJun overexpression in MSP RAS cells (Figure 5.14A) and thereupon tested the effects of the siRNA-mediated cJun knock down in MSP RAS cells. Despite an efficient cJun siRNA knock down, no changes for Bcl-xL mRNA or protein levels were observed (Figure 5.14B, C). Given the fact that AP-1 can consist of either Jun family protein homodimers or Jun-Fos heterodimers, we were interested to investigate the Bcl-xL level after siRNA-mediated knock down of combinations of cJun, cFos or JunD [134]. The knock down efficiency of cFos and JunD was defined by qRT-PCR (Figure 5.15C, D). However, when Bcl-xL protein levels were analyzed by western blot $48 \mathrm{~h}$ after knock down, no differences were visible in the knock down samples compared to the mock and scr control (Figure 5.15A). Because of the high stability of chromatin-associated transcription factors, we decided to perform a reiterated siRNA-mediated AP-1 knock down 48h after the first knock down. After 96h cells were harvested and the protein lysates were analysed for Bcl-xL. The knock down efficiency was monitored by Cyclin D1 and cJun, but no differences were detectable. It can be concluded, that the reiterated knock down was not successful. Accordingly, no changes for Bcl-xL protein levels were detectable. Since the combination of different siRNA in one experiment can cause reduced knock down efficiencies, this could be the reason for the lacking siRNA efficiencies in this experiment.

A

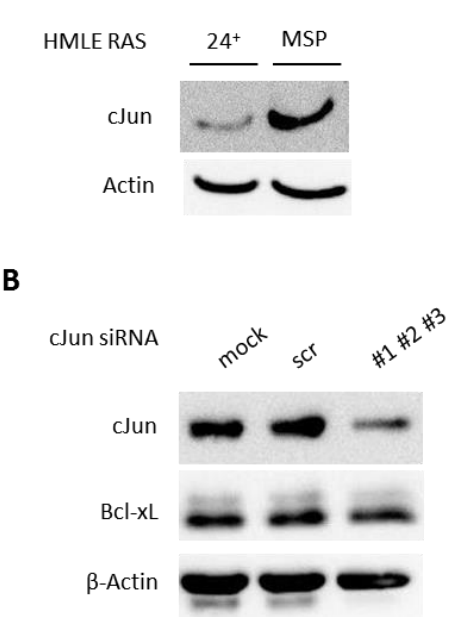

C

Bcl-xL gene expression after cJun siRNA knock down

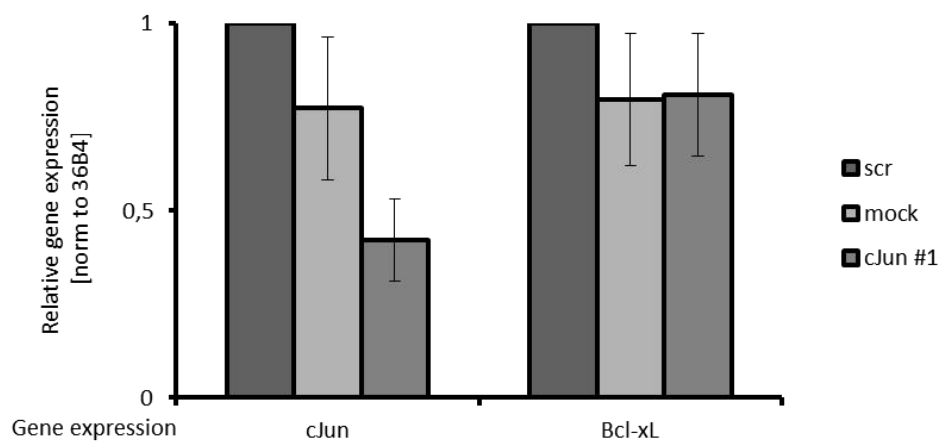

Figure 5.14: EMT results in overexpression of the transcription factor cJun, but this is not the reason for Bcl-xL accumulation.

(A) HMLE RAS cells were harvested 24h after seeding. Protein lysates were analyzed for cJun by western blotting. (B) 48h after siRNA-mediated cJun knock down MSP RAS cells were harvested and lysates were analyzed for Bcl-xL level by western blot. Actin staining was used as loading control. One representative experiment of $\mathrm{n}=3$ is shown. (C) $48 \mathrm{~h}$ after siRNA mediated cJun knock down MSP RAS were harvested. mRNA was isolated 
and mRNA levels of cJun and Bcl-xL were analyzed using qRT-PCR. mRNA levels were normalized to $36 \mathrm{~B} 4$. Non-transfected cells and scr siRNA were used as control. Data show mean \pm SD of $n=3$.

A

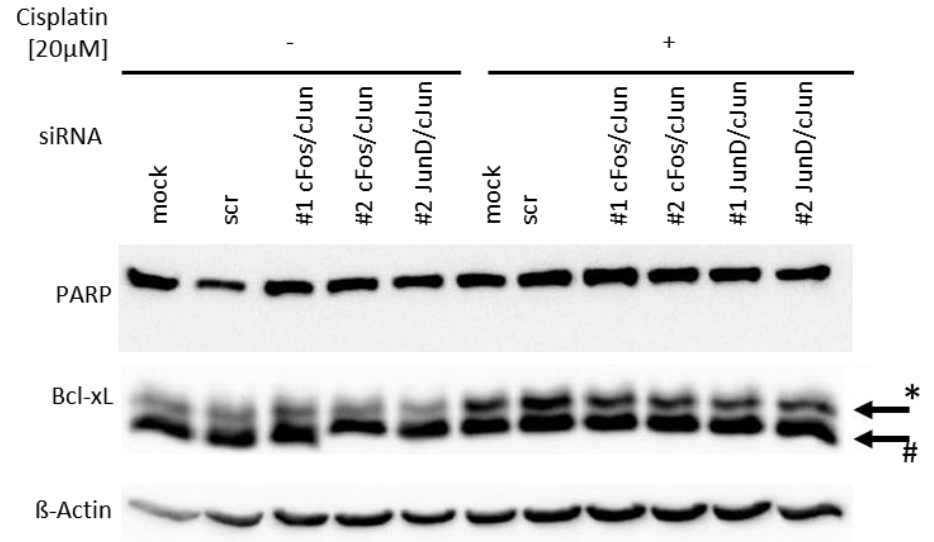

C

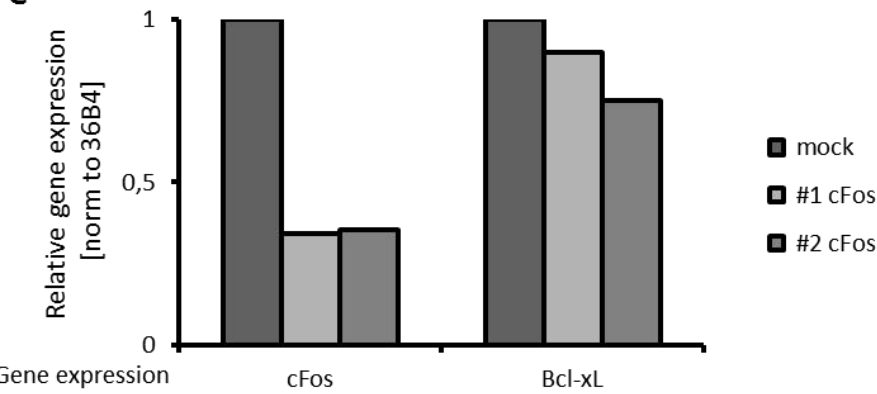

D

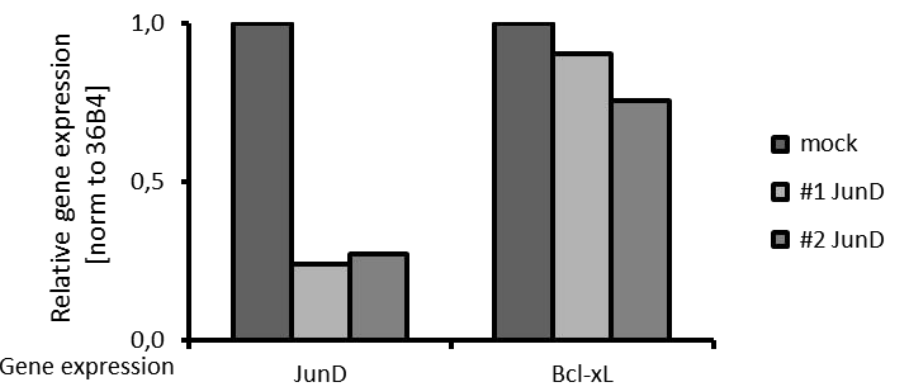

B

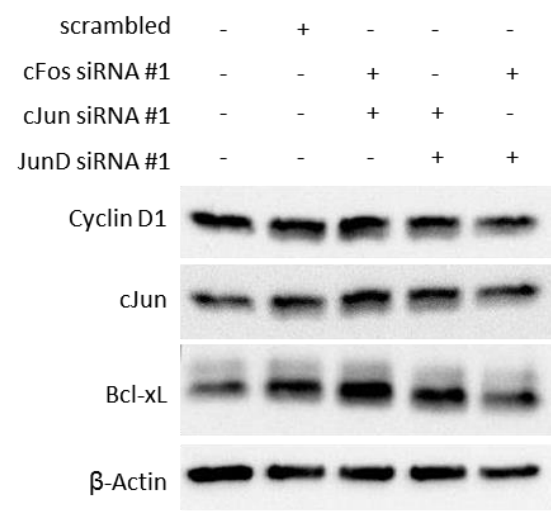

Figure 5.15: AP-1 transcription factor knock down has no detectable effect on Bcl-xL level in MSP RAS cells.

(A) 32h after siRNA-mediated knock down of AP-1 with combinations of cFos, cJun and JunD siRNA, MSP RAS cells were treated with $20 \mu \mathrm{M}$ Cisplatin for $16 \mathrm{~h}$. Subsequently, cells were harvested and lysates were analyzed by western blotting. Untreated cells were used as control. For Bcl-xL: $\leftarrow *$ indicates deamidated protein, $\leftarrow \#$ indicates unmodified protein. (B) $96 \mathrm{~h}$ after reiterated siRNA-mediated knock down of AP-1 with combinations of cFos, cJun and JunD siRNA, cells were harvested and lysates were analyzed for Bcl-xL level by western blot. Actin staining was used as loading control, while CyclinD1 staining was used as positive control for treatment. (C-D) 48h after siRNA-mediated knock down of cFos (C) or JunD (D) cells were harvested, mRNA was isolated and mRNA levels of cFos (C) or JunD (D) and Bcl-xL were determined using qRT-PCR. mRNA levels were normalized to 36B4. Non-transfected cells and scr siRNA were used as control. $n=1$. 
Besides an AP-1 binding site, the Bcl-xL gene contains transcription factor binding sites that are bound by STAT transcription factors, especially by STAT5 [133]. We therefore tested, whether STAT-mediated siRNA knock down effected Bcl-xL level. qRT-PCR analysis showed efficient knock down of STAT3 as well as STAT5a and STAT5b on mRNA level (Figure 5.16B, C). 32h after STAT siRNA knock down; MSP RAS cells were treated with $20 \mu \mathrm{M}$ Cisplatin and the induction of apoptosis as well as Bcl-xL protein level were defined by western blot analyses. The knock down efficiency of STAT siRNA $48 \mathrm{~h}$ after transfection was indirectly monitored by decreased Cyclin D1 protein level. In contrast, no changes were evident for Bcl-xL or apoptosis-induction in dependence on STAT knock down. These findings for STAT are in line with the result for the siRNA-mediated knock down of AP-1. It can be concluded that these transcription factors do not regulate the Bcl-xL level in MSP RAS cells to a detectable degree.
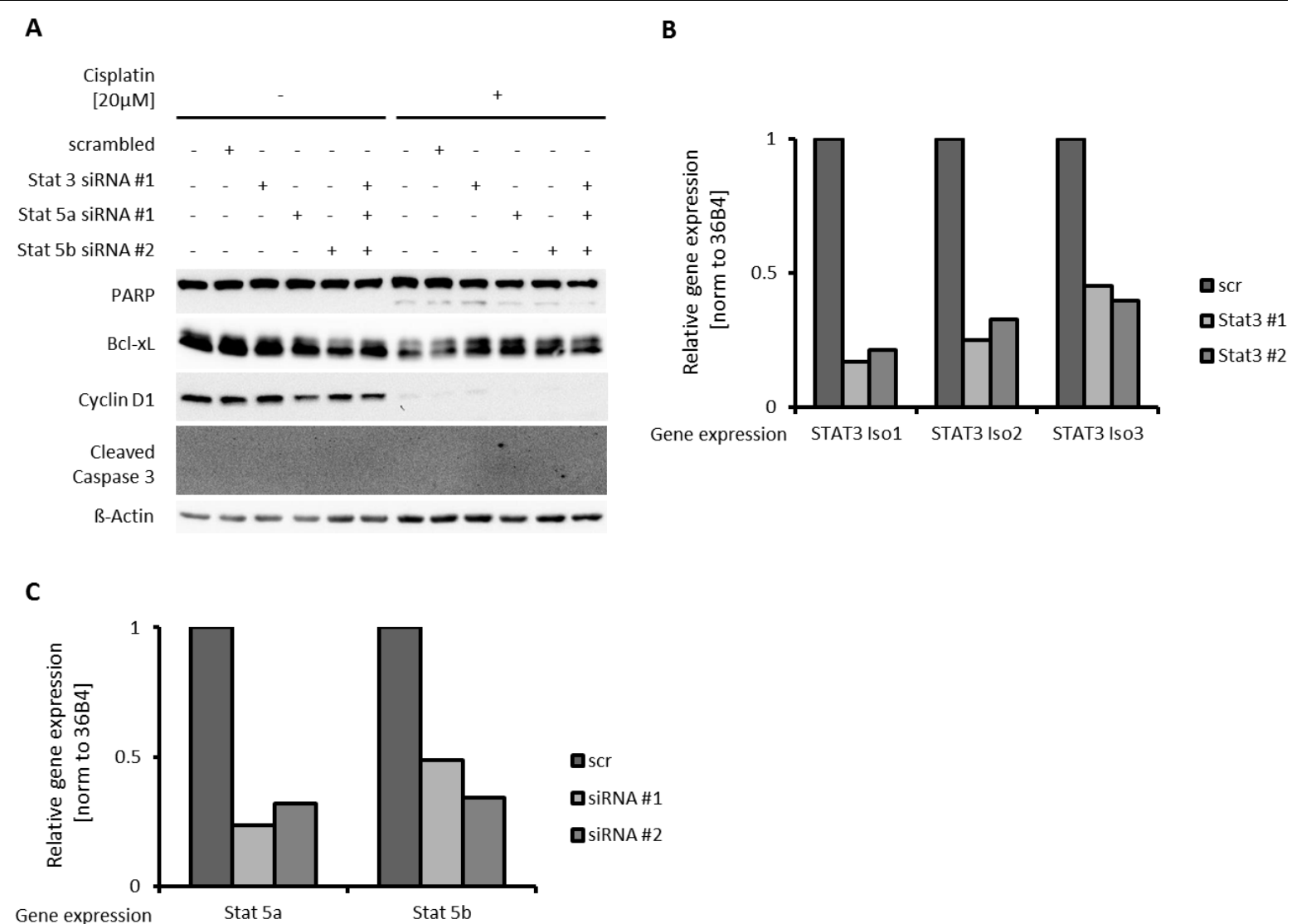

Figure 5.16: STAT siRNA knock down has no detectable effect on Bcl-xL level in MSP RAS cells.

(A) 32h after siRNA mediated knock down of STAT3, STAT5a and STAT5b, MSP RAS cells were treated with $20 \mu \mathrm{M}$ Cisplatin for 16h. Subsequently, cells were harvested and lysates were analyzed for Bcl-xL level by western blot. Actin staining was used as loading control while CyclinD1 staining was used as positive control for treatment. Untreated cells were used as control. (C-D) 48h after siRNA-mediated transfection of STAT3 (C) or STAT5a and STAT5b (D) cells were harvested, mRNA was isolated and mRNA levels of STAT3 (C) or STAT5a and STAT5b (D) were analyzed using qRT-PCR. mRNA levels were normalized to 36B4. Nontransfected cells and scr siRNA were used as control. $n=1$. 


\subsection{Overcoming the chemoresistance of MSP RAS cells by the inhibition and destabili- zation of Bcl-xL}

\subsubsection{Gossypol stops MSP RAS cell proliferation and induces autophagy}

Cells that have undergone EMT pose a challenge to cancer therapy as they develop chemotherapeutic resistance which results in metastasis and relapse of cancer disease. We therefore aimed to overcome the chemotherapeutic resistance of MSP RAS cells overexpressing Bcl$\mathrm{xL}$. To this end, we decided to use the Bcl-xL inhibitor Gossypol which mimics the Bcl-2 homology domain 3 (BH3), binds to Bcl-2 proteins and neutralizes them [135]. To investigate the effect of Gossypol on HMLE RAS cells we performed cell proliferation assays. HMLE RAS cells were treated with 6 to $30 \mu \mathrm{M}$ Gossypol for $24 \mathrm{~h}$. Subsequently, Gossypol was replaced by fresh medium and the cell proliferation was measured over $8 \mathrm{~d}$ every $24 \mathrm{~h}$ by direct cell counting, defining the cell number per well using a Celigo® cytometer. While Gossypol concentrations up to $18 \mu \mathrm{M}$ displayed no distinctive effect on HMLE RAS cell proliferation (Figure 5.17B), $24 \mu \mathrm{M}$ and $30 \mu \mathrm{M}$ Gossypol disrupted the cell proliferation of $24^{+}$RAS cells as well as MSP RAS cells with no regain of cell viability (Figure 5.17C).

When we applied Gossypol in parallel with Cisplatin for 16h to HMLE RAS it was obvious that apoptosis was still not induced in MSP RAS cells. Since Gao et al. showed that Gossypol is able to induce autophagy in cancer cells, we analyzed cell lysates for apoptosis and autophagy by western blot [135]. Thus, we examined the autophagy marker LC3a/b I. LC3a/b I is a widely used marker for autophagy because of its lipidation and recruitment to the autophagosomes after induction of autophagy in the cell. The lipidation of LC3a/b I results in LC3a/b II which displays an increase in the electrophoretic mobility of this protein on gels compared to LC3a/b I [136]. The signal for LC3a/b II is therefore below the staining for LC3a/b I in western blot. Thus, induced autophagy is visible by increased levels of LC3a/b II. Indeed, for MSP RAS cells no activation of Caspase 3 was detectable. However, the signal for LC3a/b II accumulated in MSP RAS cells upon Gossypol treatment in the presence or absence of Cisplatin (Figure 5.18). 


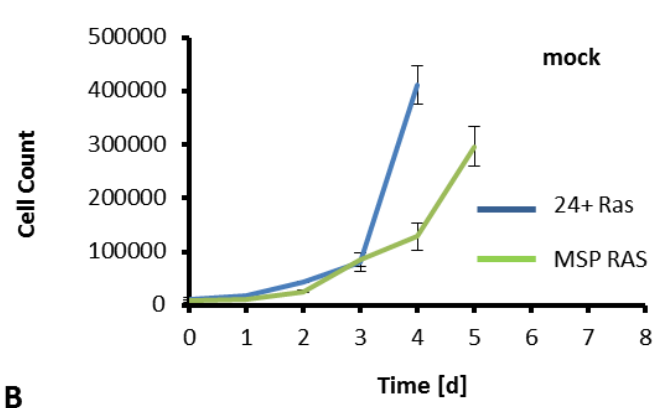

B
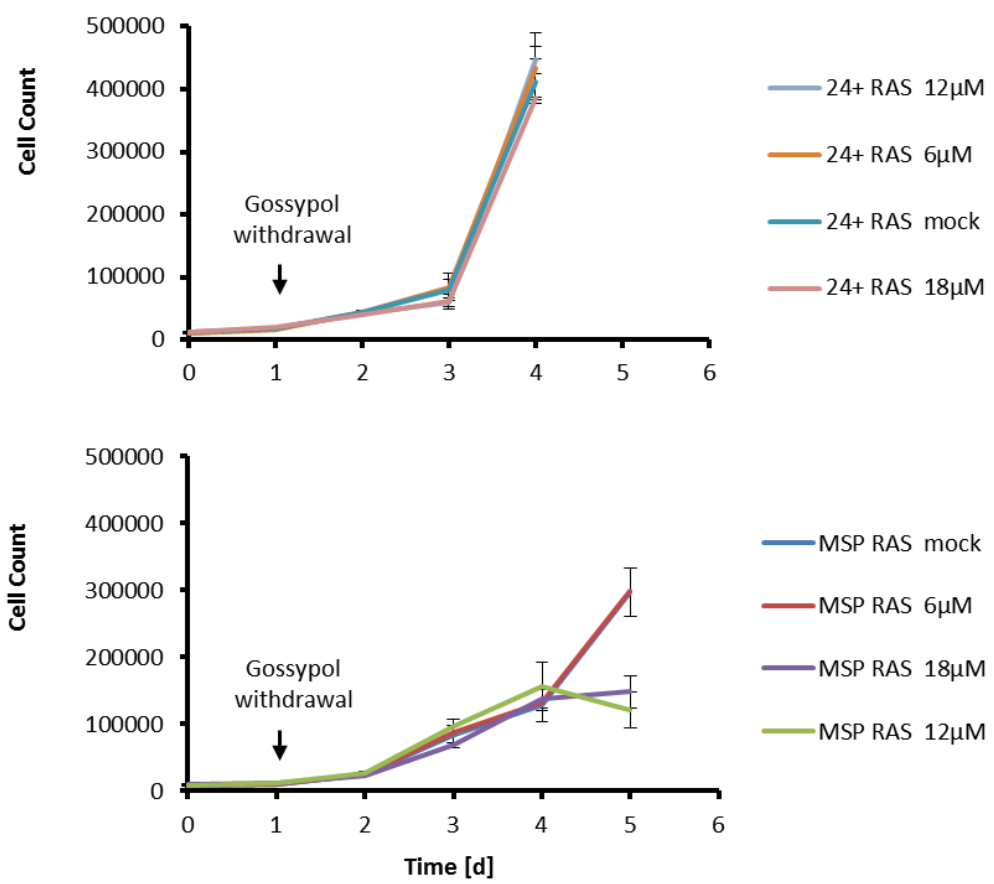

C
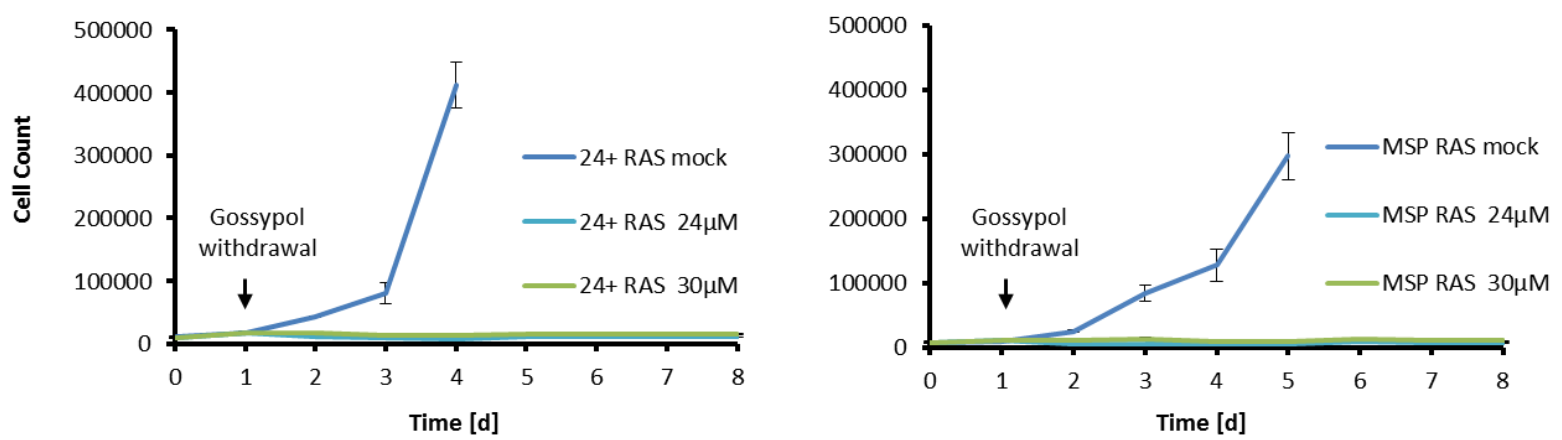

Figure 5.17: The Bcl-xL inhibitor Gossypol stops proliferation of MSP RAS cells.

(A) HMLE RAS cells were seeded in duplicates in 12-well plates. 4h after seeding direct cell counting was performed by defining the cell number/well with a Celigo ${ }^{\circledR}$ cytometer for the indicated time periods. (B, C) $24 \mathrm{~h}$ af-

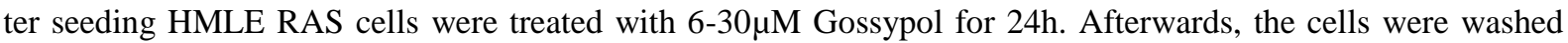
with supplemented MEGM medium and kept in culture. Cell number was measured once a day for 8 days with a Celigo ${ }^{\circledR}$ cytometer after adding fresh medium. DMSO-treated cells were used as control. Data show mean \pm SD. One representative experiment of $n=2$ is shown. 


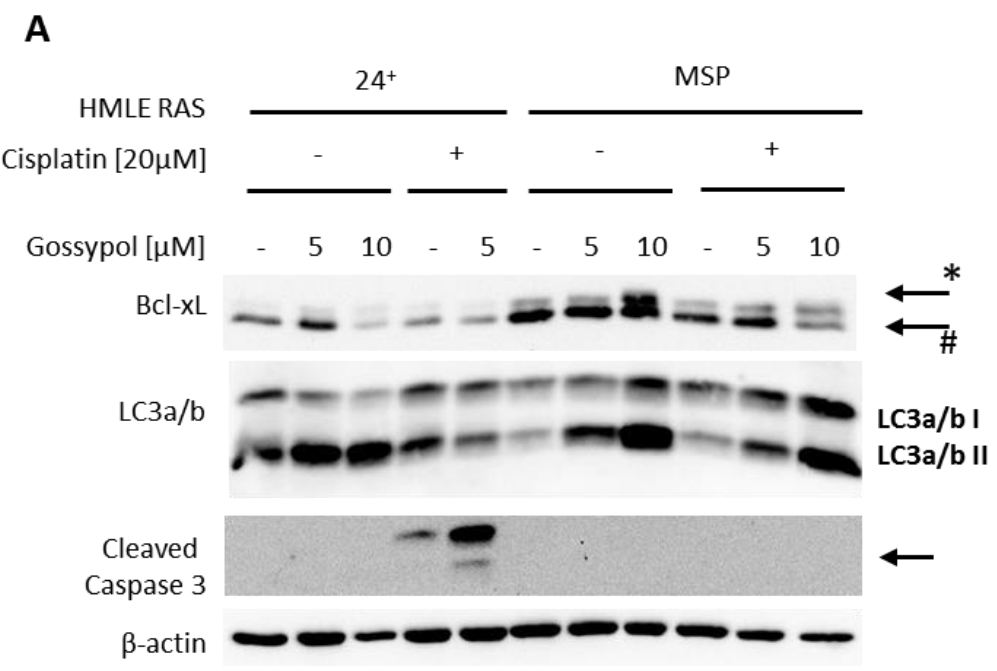

Figure 5.18: The Bcl-xL inhibitor Gossypol induces autophagy in MSP RAS cells.

16h after seeding HMLE RAS cells were exposed to $5 \mu \mathrm{M}$ or $10 \mu \mathrm{M}$ Gossypol in the presence or absence of Cisplatin for $16 \mathrm{~h}$. Afterwards, cells were harvested and lysates were analyzed for apoptosis by western blotting. Actin staining was used as loading control, while LC3a/b staining was used as indicator for autophagy. Accumulation of LC3a/b II indicates autophagy. Non-treated and DMSO-treated cells were used as control. Arrows are indicating the intended staining. For Bcl-xL: $\leftarrow *$ indicates deamidated protein, $\leftarrow \#$ indicates unmodified protein. $\mathrm{n}=1$.

\subsubsection{HSP90 inhibition destabilizes Bcl-xL and induces apoptosis in MSP RAS cells}

As Caldas-Lopes et al. have shown that Bcl-xL is a client of HSP90 and is therefore protected from proteasomal degradation [137], we were interested to see, whether HSP90 inhibition can destabilize Bcl-xL and may this affect the response to Cisplatin treatment. For this, we made use of the HSP90 inhibitors 17AAG as well as 17DMAG which disable the ATP binding site of HSP90 and thereby abolish HSP90 function [138]. MSP RAS cells were treated with HSP90 inhibitors for $24 \mathrm{~h}$ followed by exposure to Cisplatin for 16h. Subsequently, the protein lysates were analyzed for Bcl-xL and induced apoptosis by western blot. We found that the inhibition of HSP90 in the absence of Cisplatin induced a decrease of Bcl-xL protein level associated with active Caspase 3 and cleaved PARP. The induction of apoptosis was enhanced in the presence of Cisplatin. The decrease of Akt protein level indicated the efficiency of the HSP90 inhibitors and is well detectable in the presence or absence of Cisplatin (Figure 5.19A). Next, it was tested whether the overexpression of Bcl-xL in $24^{+} \mathrm{HMLE}$ cells and MSP cells rescued the induction of apoptosis mediated by HSP90 inhibition. Indeed, neither in the absence nor in presence of Cisplatin apoptosis was induced in HMLE overexpressing Bcl-xL (Figure 5.19B). 
It can be concluded that the inhibition of HSP90 results in a decrease of Bcl-xL level which in turn sensitizes MSP RAS cells to Cisplatin treatment. The high Bcl-xL levels in expression vector-containing HMLE cells rescued these cells from the induction of apoptosis mediated by HSP90 inhibition. This implicates that Bcl-xL plays a role in this apoptosis induction.

A

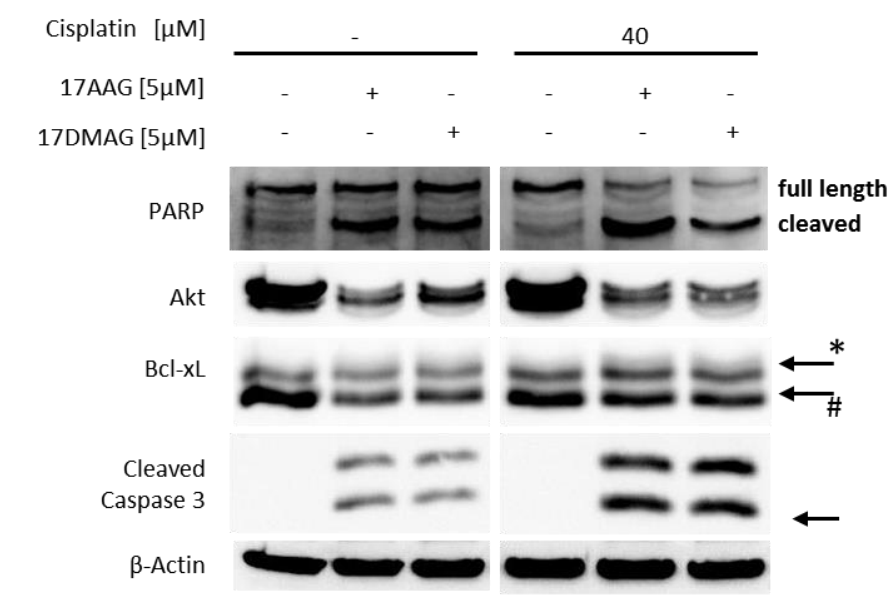

B

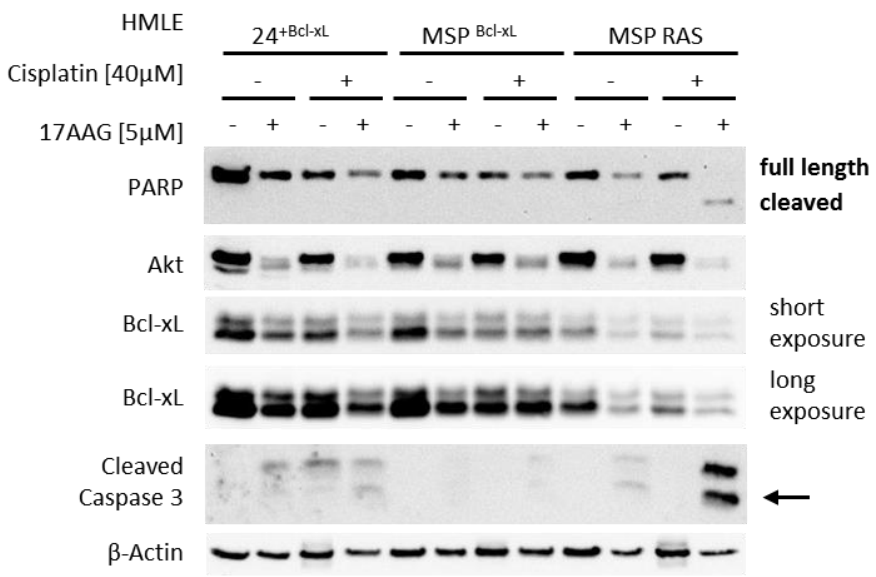

Figure 5.19: HSP90 inhibition sensitizes MSP RAS cells towards Cisplatin.

(A) $16 \mathrm{~h}$ after seeding MSP RAS cells were treated with 5 $\mathrm{MM}$ 17AAG or 17DMAG for 24h. Subsequently, cells were washed and exposed to $20 \mu \mathrm{M}$ Cisplatin for $16 \mathrm{~h}$. Thereafter, cells were harvested and lysates were analyzed for apoptosis and Bcl-xL by western blot. $\leftarrow *$ indicates deamidated protein, $\leftarrow \#$ indicates unmodified protein. $\mathrm{n}=3$. (B) $16 \mathrm{~h}$ after seeding 24+Bcl-xL, MSP Bcl-xL and MSP RAS were treated with 5 $\mu$ M 17AAG for 24h. Afterwards, the cells were washed and treated with $40 \mu \mathrm{M}$ Cisplatin for $16 \mathrm{~h}$. Then, cells were harvested and analyzed for apoptosis and Bcl-xL by western blot. $\mathrm{n}=1$. Actin staining was used as loading control, while Akt staining was used as positive control for the experiment. DMSO-treated cells were used as control. Arrows are indicating the intended staining.

Interestingly, the HSP90 inhibition with 17AAG and 17DMAG resulted in decreased Bcl-xL mRNA level, as well (Figure 5.20A). To examine, whether HSP90 directly causes a decrease in Bcl-xL mRNA level we performed a siRNA-mediated knock down of HSP90 and analyzed the Bcl-xL mRNA level 48h post-transfection with qRT-PCR. It was detectable that HSP90 
knock down results in decreased Bcl-xL mRNA level, as well. However, this decrease was less prominent than that induced by 17AAG-mediated HSP90 inhibition (Figure 5.20B). These data implicate that not only HSP90-mediated direct Bcl-xL protein stabilization is important for the Bcl-xL level in MSP RAS cells. Rather, the stabilization of other HSP90 clients affects Bcl-xL gene expression in these cells as well.

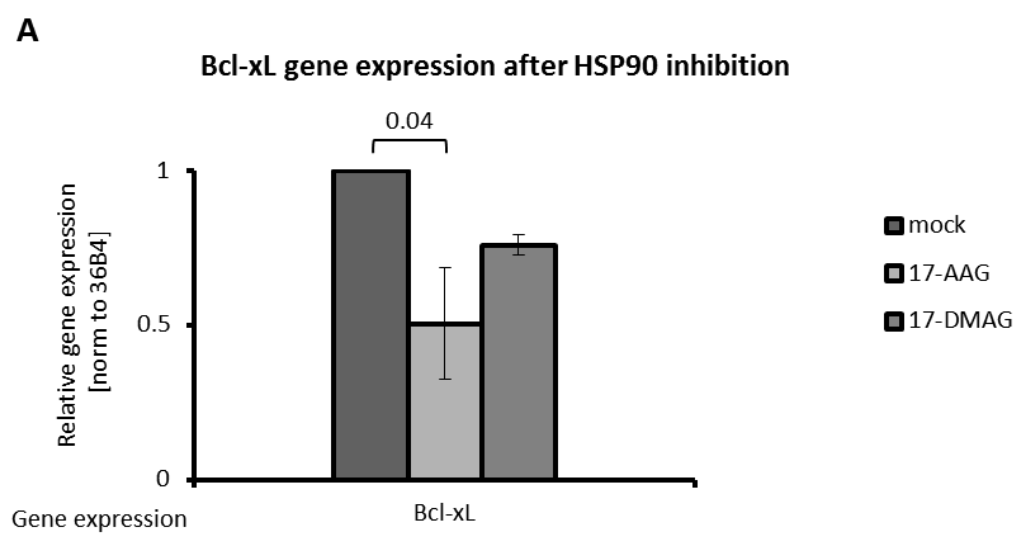

B

Bcl-xL gene expression after HSP90 siRNA knock down

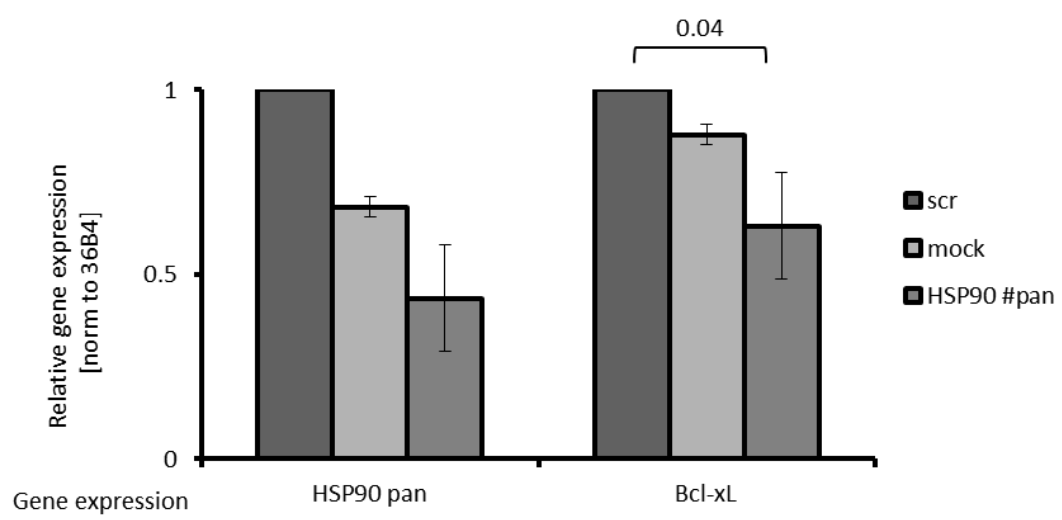

Figure 5.20: HSP90 inhibition decreases Bcl-xL mRNA level.

(A) 16h after seeding MSP RAS cells were treated with 5 $\mathrm{MM}$ 17AAG or 17DMAG for 24h. Subsequently, cells were harvested and mRNA was isolated. mRNA levels of Bcl-xL were analyzed using qRT-PCR. DMSO-treated cells were used as control. (B) 48h after siRNA-mediated knock down of HSP90 $\alpha$ and HSP90 $\beta$, cells were harvested, mRNA was isolated and mRNA levels of HSP90pan and Bcl-xL were analyzed by qRT-PCR. Nontransfected cells and scr siRNA were used as control. mRNA levels were normalized to 36B4. siRNA data show mean $\pm \mathrm{SD}$ of $\mathrm{n}=3$. Student's t-test was performed for statistical analyses.

\subsubsection{HSP90 inhibition reduces MSP RAS cell-derived tumors in mice}

Previous results suggest the possibility to overcome the chemoresistance of MSP RAS cells by inhibiting HSP90 with 17DMAG. To this end, we were interested to examine whether MSP RAS cell-derived tumors in mice respond to 17DMAG under physiological conditions 
as it was the case for MSP RAS cells in vitro. To test this, MSP RAS cells were injected into three week old $(\mathrm{Nu} / \mathrm{Nu})$ mice. In the phase of exponential tumor growth three weeks after injection, xenograft mice were treated with intraperitoneal injection of 17DMAG 5d per week until sacrifice. Tumor size was defined every day and plotted against time. This first experiment demonstrated that, 17DMAG treatment resulted in reduced tumor sizes compared to the control mice. This effect was reduced during the rest of the treatment (Figure 5.21).

These results support the notion that HSP90 inhibition constitutes a possibility to overcome chemoresistance in MSP RAS cells. Moreover, these findings suggest that this is even possible under physiological conditions in mice. However, these first data have to be verified with a larger number of mice.

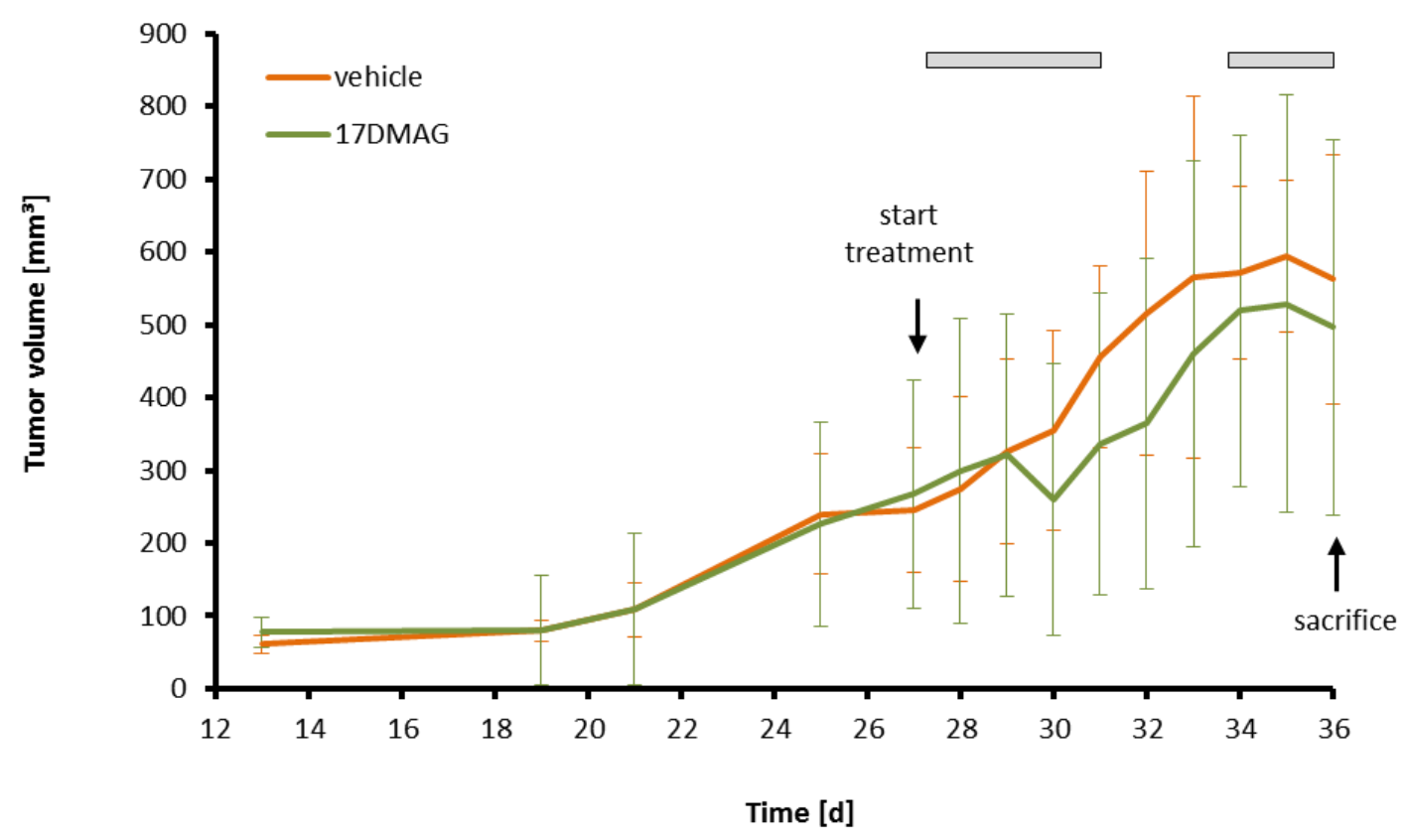

Figure 5.21: HSP90 inhibition via systemic 17DMAG treatment induces growth inhibition of MSP RAS cell-induced tumors in mice.

$10^{6}$ MSP RAS cells were subcutaneously injected in $(\mathrm{Nu} / \mathrm{Nu})$ mice. Three weeks after injection, xenograft mice were treated with intraperitoneal injections of $10 \mathrm{mg} / \mathrm{kg} /$ day $17 \mathrm{DMAG}$ (green line) or vehicle $(5 \%$ glucose, orange line) $5 \mathrm{~d}$ per week until sacrifice. During treatment, tumor sizes were monitored every day. (Preliminary data; 4 tumors control group; 6 tumors $17 \mathrm{DMAG}$ group). Data show mean $\pm \mathrm{SD}$. 


\section{Discussion}

The developmental program of Epithelial Mesenchymal Transition (EMT) appears to enable cancer cells to disseminate from the primary tumor and form metastasis in distant tissues of the organism. In addition, EMT has become a prominent process in the acquisition of chemoresistance and cancer regrowth in patients associated with a poor prognosis. In order to investigate about how EMT results in chemotherapeutic resistance we were provided with the HMLE and HMLE RAS model systems for EMT by Weinberg and colleagues (MIT Boston, USA), consisting of the $24^{+}$HMLE and MSP cell lines and the $24^{+}$RAS and MSP RAS cell lines, respectively.

Our results demonstrated that MSP and MSP RAS cells are resistant to chemotherapeutic treatment, including Cisplatin, Carboplatin, Doxorubicin and Neocarzinostatin. Most strikingly, we found that MSP and MSP RAS cells acquire the ability to prevent the induction of apoptosis, through intrinsic or extrinsic apoptosis signalling pathway. In particular, we identified the anti-apoptotic Bcl-2-family member Bcl-xL to be overexpressed in MSP as well as MSP RAS cells, which causes apoptosis-resistance. Inhibition of Bcl-xL with the BH3 mimic Gossypol resulted in chemosensitivity in MSP RAS cells. The same observation was obtained upon destabilization of Bcl-xL, mediated by inhibition of HSP90 chaperone function in MSP RAS cells in vitro and in vivo. Thus, our results point out a potential strategy to overcome chemoresistance in cells that have undergone EMT.

In conclusion, Bcl-xL appears to be a key factor of apoptosis in cells upon EMT. We propose a causal relation between EMT, chemoresistance and Bcl-xL overexpression, and we suggest Bcl-xL as a possible target in cancer therapy (Figure 6.1). 


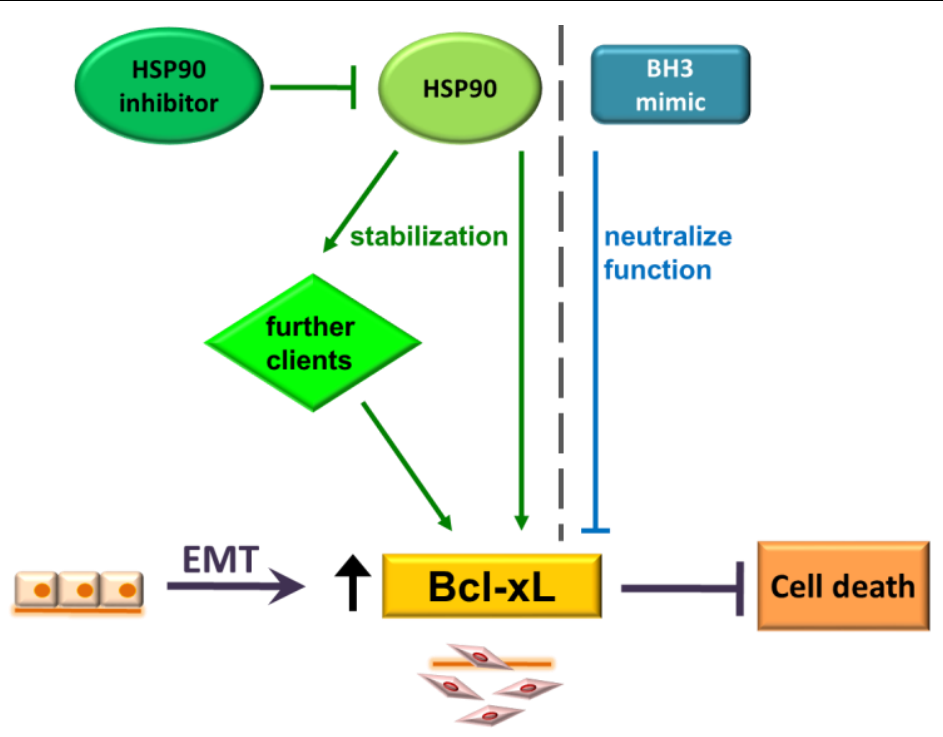

Figure 6.1: EMT renders HMLE and HMLE RAS cells resistant to chemotherapy by the overexpression of Bcl-xL.

EMT results in the up-regulation of the anti-apoptotic protein $\mathrm{Bcl}-\mathrm{xL}$, which in turn prevents cell death in cells that have undergone EMT. The inhibition of the chaperone HSP90 affects Bcl-xL stabilization, which results in decreased Bcl-xL level and induced apoptosis. However, further HSP90 clients are involved in Bcl-xL transcriptional regulation, which can result in decreased Bcl-xL protein level and induced apoptosis upon HSP90 inhibition as well. The BH3 mimic Gossypol associates with the BH3-domain of Bcl-2-family members and neutralizes their function. Thus, Gossypol treatment lead to cell death in MSP RAS cells.

\subsection{EMT renders HMLE and HMLE RAS cells resistant to chemotherapy}

\subsubsection{Other examples of EMT correlating with chemoresistance}

The process of EMT in cancer cells is not only associated with an invasive and migratory phenotype. Rather, EMT results in cancer cells that gain the ability to escape conventional chemotherapy by acquiring chemoresistance [85]. Based on this knowledge we asked whether EMT affects the response to chemotherapeutics in the HMLE and HMLE RAS system and treated them with Cisplatin, Carboplatin, Doxorubicin and Neocarzinostatin. Indeed, we found that apoptosis was activated in $24^{+} \mathrm{HMLE}$ and $24^{+}$RAS cells, but not in MSP or MSP RAS cells, displayed by cleaved Caspase 3 and cleaved PARP (Figure 5.2A-C, Figure 5.6AC). Consistent with our findings, Tryndyak et al. defined a correlation between EMT in the human breast cancer cell lines MDA-MB-231 and BT-549, and chemoresistance to Doxorubicin [139]. Correspondingly, Asiedu et al. have shown that induced EMT in the epithelial mouse mammary carcinoma cell line MMC resulted in mesenchymal cells (ETTM) that were resistant to Oxaliplatin, Taxol and Etoposide [140]. Additionally, Arumugam et al. observed 
that EMT mainly triggered by the overexpression of the transcription factor Zeb1 in these cell lines renders human pancreatic cancer cell lines multidrug resistant to Cisplatin, Gemcitabine and 5'-FU [141]. Similar findings were observed for Zeb2-overexpressing bladder cancer cells, which are resistant to UV- and Cisplatin-induced apoptosis, distinguished by the lack of activation of Caspase 3 and cleaved PARP [142]. These results indicated that the induction of EMT leads to chemoresistance in several cell lines as well as in our EMT model systems, and results in the failure to induce apoptosis upon treatment.

\subsubsection{Impact of cell proliferation and drug uptake}

To stress a possible mechanism for the chemotherapeutic resistance, we initially investigated the cell proliferation of cells before and upon EMT. It was recently published that EMT activators are concomitant with impaired proliferation activity, which is assumed to protect cells from DNA damage and would result in delayed apoptosis induction after chemotherapeutic treatment $[122 ; 123]$. We therefore wanted to define whether different growth rates correlate with to the failure of apoptosis induction in MSP and MSP RAS cells. In fact, our results show that MSP cells displayed decreased growth rates compared to the $24^{+}$HMLE cells after Cisplatin treatment. However, the growth rates of $24^{+}$RAS and MSP RAS cells were comparable (Figure 5.3A, B mock). We assumed that the latter observation is caused by the overexpression of the RAS-oncogene, which is known to increase cell proliferation by induction of Cyclin D1 and might therefore be mediating similar growth behavior in the HMLE RAS cell system [143]. Since we observed no induction of apoptosis after Cisplatin treatment in both cell lines, MSP and MSP RAS, upon EMT, we concluded that different proliferation rates are not the reason for this finding. Strikingly, when we treated the cells with Cisplatin for $24 \mathrm{~h}$ followed by prolonged incubation, we observed that MSP RAS cells continued their proliferation after 5d, while $24^{+}$RAS cells did not recover (Figure 5.3B). Considering these findings, we asked whether MSP RAS cells gain the possibility to escape Cisplatin treatment by increased drug efflux. We therefore determined the amount of adducts formed by Cisplatin and DNA in HMLE RAS cells. However, we did not detect differences between $24^{+}$RAS and MSP RAS cells (Figure 5.4). Surprisingly, it is especially known for Cisplatin that the ectopic expression of the Cu-transporter ATP7A and 7B is associated with decreased Cisplatin sensitivity because of increased Cisplatin export [37]. Beyond that, the surface marker CD44 contributes to drug resistance via the interaction with the $\mathrm{ABC}$-transporter P-glycoprotein, which is involved in drug efflux, as well $[34 ; 144]$. The expression of CD44 is assumed to induce a metastatic phenotype in locally growing tumor cells and is highly induced in MSP and MSP 
RAS cells [110; 145]. However, our finding that both cell lines contained comparable amounts of Cisplatin adducts after treatment suggests that Cisplatin transport processes are not involved in apoptosis resistance of MSP RAS cells.

In summary, we have shown that EMT results in MSP and MSP RAS cells which are resistant to distinct chemotherapeutics. Further, we demonstrated that neither differences in the cell proliferation nor drug efflux protect MSP RAS cells from chemotherapy.

\subsubsection{Manipulation of EMT-associated pathways fails to sensitize MSP RAS cells}

EMT is executed by the activation and modification of distinct signalling pathways that might result in chemoresistance. On the one hand, an active Wnt-pathway is associated with EMT, marked by phosphorylated JNK and, subsequently, overexpression of Jun and active p38 $[110 ; 126]$. On the other hand, TGF $\beta$ plays a crucial role in the process of EMT [85]. To assess the relevance of these pathways in cell death resistance in our HMLE RAS system, we made use of respective inhibitors in the presence of Cisplatin. We found that the inhibition of JNK and p38 as downstream effectors of the Wnt-pathway exhibit no effect on the response of MSP RAS cells to Cisplatin. The same observation was obtained upon inhibition of Mek1/2 and the TGF $\beta$ pathway (Figure 5.5B-D). In contrast to our results, Vangipuram et al. suggested a correlation between the active Wnt-pathway and chemoresistance in neuroblastoma cell lines, as they successfully sensitized these cells to Doxorubicin by the application of specific Wnt-inhibitors [146]. Similar results have been found for chemoresistant human colon cancer cells by Chikazawa et al., whereby the siRNA-mediated knock down of $\beta$-Catenin diminished Wnt-signalling and retrieved sensitivity of these cells to Paclitaxel [147]. Further, it has been demonstrated with cell culture experiments that TGF $\beta$ treatment resulted in induction of reversible EMTs. Thereby, TGF $\beta$ withdrawal immediately results in the reversion from a mesenchymal to an epithelial phenotype [148]. In contrast to our findings, the blockage of Mek1/2 resulted in inactivation of the Erk1/2 kinases and inhibition of TGF $\beta$-induced EMT in NMUMG cells [149]. Interestingly, Scheel et al. have previously shown that TGF $\beta$ and Wnt-molecules, such as Wnt5a, are essential for the EMT induction in $24^{+}$HMLE, indicating that the Wnt- and TGF $\beta$ signalling pathways are indispensible for the EMT in the present model system [110]. However, our findings suggest that inhibition of TGF $\beta$ and Wntpathways are not sufficient to induce chemosensitivity in MSP RAS cells. Given the fact that the MSP as well as MSP RAS cells in our model system reside stably in their mesenchymal state [110], we assume that we are working with an EMT system that is associated with largely irreversible epigenetic changes. Thus, we conclude that manipulation of EMT-inducing 
molecules, such as Wnt or TGF $\beta$, for short time periods is insufficient to revert EMT-induced cellular changes that cause chemoresistance.

\subsubsection{MSP RAS cells are resistant to extrinsic apoptosis induction}

We have shown that EMT leads to chemoresistant MSP and MSP RAS cells. However, the most striking observation was that MSP RAS cells failed to undergo apoptosis independent of the chemotherapeutic treatment we applied. Additionally, we demonstrated that the exposure of HMLE RAS cells to death ligands, such as Trail and TNF $\alpha$, results in the induction of apoptosis in $24^{+}$RAS cells, but not in MSP RAS cells, indicating that Caspase 8 and Caspase 3 can only be activated downstream of the mitochondria in these cells (Figure 5.7A, B). Consistent with our findings, it has been recently published that acquisition of multidrugresistance upon EMT correlates with altered death receptor signalling [150]. Antoon et al. have shown that resistance of the MCF7 breast cancer cell line to TNF $\alpha$ is related to EMT and an altered death receptor pathway, blocking extrinsic apoptosis. They hypothesized that altered TNF receptor expression switched TNF ligand binding from cell death to pro-survival signals in these cells. These changes made MCF7 cells resistant to the clinical chemotherapeutic agents Trail, Etoposide, Paclitaxel and Doxorubicin and was accompanied by increased tumorigenesis [150]. Interestingly, a very recent report demonstrated that the gene expression of FAS and TNF signalling mediators was down-regulated by the anti-apoptotic protein Bcl$\mathrm{xL}$, which is a common protein involved in chemoresistance [151; 152]. In this context, the next step is to investigate whether the protein level of death receptors, mediating extrinsic apoptosis, are differentially present in MSP RAS compared to $24^{+}$RAS cells. Further, it is known that increased expression of anti-apoptotic Bcl-2 family members, such as Bcl-2 and Bcl-xL, and decreased expression pro-apoptotic Bcl-2 family members, like Bax and Bid, represent prominent resistance mechanisms that disrupt mitochondria-initiated apoptosis in breast cancer $[150 ; 152]$. However, as of yet, these findings have not been directly linked to EMT. Taken together, the failure to induce apoptosis in cells that have undergone EMT raises the idea that EMT results in changes to the downstream cellular machinery involved in apoptosis. 


\subsection{The anti-apoptotic protein Bcl-xL as a mediator of chemoresistance in cancer cells}

\subsubsection{Bcl-xL protects MSP RAS cells from apoptosis}

Within our studies we observed that the anti-apoptotic Bcl-2-family member Bcl-xL is overexpressed in MSP as well as MSP RAS cells compared to their parental counterpart $24^{+}$HMLE and $24^{+}$RAS cells, respectively (Figure 5.8A-C). Correspondingly, we have demonstrated that the overexpression of Bcl-xL in $24^{+}$HMLE renders these cells resistant to chemotherapy, as well as to the initiation of extrinsic apoptosis via treatment with Trail (Figure 5.10). Conversely, siRNA-mediated knock down of Bcl-xL in MSP RAS cells rendered them sensitive to death stimuli (Figure 5.9A-C). Consistent with our observations, Kunze et al. have shown that not only the siRNA-mediated knock down of Bcl-2, Bcl-xL, but also the reduction of XIAP (X-linked inhibitor of apoptosis) or survivin, sensitize chemoresistant bladder cancer cells to chemotherapy, including mitomycin $\mathrm{C}$ and Cisplatin treatment [153]. Knockout studies revealed important roles for the Bcl-2 family members Bcl-2, Bcl-xL and Mcl1 in oncogenesis [35]. However, upon EMT we were not able to detect differences in the expression of anti-apoptotic proteins, except Bcl-xL (Figure 5.8D). The first direct evidence that elevated Bcl-xL levels play an important role in cancer was demonstrated by Packham et al. in 1998 in murine leukemia cells [154]. In the meantime, overexpression of Bcl-xL was found in several cancers, such as hepatocellular carcinoma, prostate cancer, colorectal cancer and also breast cancer [76; 79]. Fernández et al. induced plasmid-mediated Bcl$x L$ overexpression in human breast cancer cell line MDA-MB-435, which resulted in an increase in the metastatic potential of these cells, an attribute that is also found in MSP RAS cells $[80 ; 110]$. Correspondingly, Martin et al. have shown that Bcl-xL expression did not induce tumor formation, but enhanced metastatic potential in breast tumor cell lines [155]. Consistent with these findings, Bcl-xL overexpression counteracts the pro-apoptotic signals released in the microenvironment of primary and disseminated tumors and promotes successful development of metastasis in the organism [156]. These data support our findings of Bcl-xLmediated chemoresistance in MSP RAS cells.

We further have demonstrated that Bcl-xL protein levels were heterogeneous within the investigated breast cancer samples, as well. The presence of different Bcl-xL protein levels did not correlate with breast cancer subtypes or the parameters Her2-status, hormone receptors, stage, sex and age (Figure 5.11A, B) [personal communication A. Scheel]. Interestingly, six investigated cases displayed pronounced $\mathrm{Bcl}-\mathrm{xL}$ protein level in tumor cells at the tumorstroma-interface and in disseminated tumor cells, supporting previously published results that 
$\mathrm{Bcl}-\mathrm{xL}$ is associated with metastatic potential (Figure 5.11A-C). Consistent with our findings, Olopade et al. examined 42 human invasive breast cancer samples for their expression of Bcl$\mathrm{xL}$ by immunohistochemical and immunoblot methods [157]. Their studies revealed that $43 \%$ of these samples contain increased levels of Bcl-xL. The overexpression of Bcl-xL in these tumors was associated with higher tumor grade and decreased overall survival. Additionally, they found no correlation between age, tumor size, ER-status or p53-status, which correlates well with our results.

We furthermore determined that the disseminated cells in our breast cancer samples and the occurrence of the mesenchymal marker N-Cadherin partly overlapped with Bcl-xL expression [A. Scheel, personal communication], which probably indicates phenotypic changes resembling an EMT-like process. Hitherto, the correlation between EMT and the overexpression of anti-apoptotic proteins has been shown for Bcl-2, but not for Bcl-xL. There is significant evidence for the association of the prevalence of CD44 in breast cancer samples with increased Bcl-2 expression, indicating that the $\mathrm{CD} 44^{+}$-population of breast cancer cells is potentially more resistant to apoptosis [158]. Additionally, given the fact that primary human mammary epithelial cells (HMECs, without large T-antigen) did not display an up-regulation of antiapoptotic proteins in comparison to human embryonic stem cells (hESCs) and human cancer cells, we suggest that the up-regulation of Bcl-xL in MSP and MSP RAS cells, originating from HMLE, is correlated with the process of EMT [159].

In conclusion, our data demonstrate Bcl-xL as a key factor of inhibition of apoptosis in our EMT systems. Further, the expression of Bcl-xL appears to be increased in a significant fraction of invasive breast cancers [157], however the general role for Bcl-xL in EMT in breast cancer requires the investigation of a larger collective to be strongly proven.

\subsubsection{Bcl-xL level in MSP RAS cells are not detectably regulated by miRNAs or candi- date transcription factors}

miRNAs play an important role in the process of EMT, particularly the families let-7 and miR-200. While the loss of let-7 in cancer results in reverse embryogenesis and dedifferentiation, miR-200 has been shown to be a prominent regulator of EMT [130]. Based on these findings, we examined the presence of miRNA let-7a, let-7b and let-7f in MSP RAS cells. However, our results demonstrated that the levels of those miRNAs did not differ in $24^{+}$RAS compared to MSP RAS cells (Figure 5.13A). In contrast to our results, Li et al. have shown that the expression of miR-200b, miR-200c, let-7b, let-7c, let-7d and let-7e were significantly down-regulated in chemoresistant pancreatic cancer cells, which showed EMT characteristics 
[160]. Further, they observed that the up-regulation of miR-200 results in the reversion of EMT and increased chemo-sensitivity in Gemcitabine-resistant MiaPaCa-2 pancreatic cancer cells. Beyond that, Shimizu et al. characterized the let-7 family as inhibitors for Bcl-xL gene expression [79]. They have shown that Bcl-xL is overexpressed in human hepatocellular carcinoma associated with increased survival. The authors defined with reporter assays a direct post-transcriptional regulation, involving miRNA let-7 and the 3'UTR of Bcl-xL mRNA. Shimizu et al. have further demonstrated that low expression of let-7 miRNA was accompanied by high Bcl-xL level, while induced let-7 expression resulted in down-regulation of Bcl$\mathrm{xL}$ and induced apoptosis after chemotherapy [79]. In contrast to our data, these results indicate a role for miR let-7 in the regulation of Bcl-xL gene expression.

To exclude that Bcl-xL levels are regulated by other miRNAs than specifically determined in TaqMan gene expression assays, we performed a siRNA-mediated knock down for Dicer, a component which is necessary for the maturation of pre-miRNA to miRNA. In the case of miRNA-mediated regulation of Bcl-xL, we expected an increase in the level of Bcl-xL in $24^{+}$ RAS cells after Dicer knock down. However, Dicer knock down affected neither the level of Bcl-xL in $24^{+}$RAS nor in MSP RAS cells (Figure 5.13B, C). Given these facts, we have not confirmed previous findings that Bcl-xL is regulated by miRNAs. Even though we did not examine all members of the miR let-7 family, we conclude from the Dicer knock down experiment that neither miRNA let-7 nor other miRNAs play a significant role for the Bcl-xL expression in our cell system.

Further, it has been shown that the Bcl-xL promoter region contains several transcription factor binding sites, including sequences for the binding of the transcription factors AP-1, STAT, Ets and $\mathrm{NF \kappa B}$, implicating various regulatory mechanisms [72; 76]. We therefore subsequently determined the effects of the transcription factors AP-1 and STAT3/5 on Bcl-xL level in MSP RAS cells. We have shown that siRNA-mediated knock down of AP-1 via the reduction of cJun, cFos and JunD did not affect Bcl-xL protein level (Figure 5.14B, Figure 5.15A-D). The same observation was obtained for siRNA-mediated knock downs of STAT3 and STAT5, which did not result in altered Bcl-xL mRNA or protein levels either (Figure 5.16AC). In contrast to our findings, Salameh et al. demonstrated the presence of two functional AP-1 binding sites in the Bcl-xL promoter which can be recognized by the heterodimer cJun:ATF2 [161]. Silencing of cJun or ATF2 resulted in decreased Bcl-xL level [161]. Further, STAT3 and STAT5 are especially known to be involved in the regulation of Bcl-xL gene expression $[72 ; 76]$. However, our data suggest that diminished levels of the transcription fac- 
tors AP-1 and STAT3/5 have no impact on Bcl-xL protein level. On the one hand, it is possible that these transcription factors are quite stable, because of their association to the chromatin. This would require the performance of extended knock down or stable knock down experiments, mediated by shRNA instead of siRNA. On the other hand, combinations of siRNAs can evoke reduced knock down efficiencies and effects on the target proteins. Thus, we are going to verify these results with single instead of combined siRNA knock downs to make our final conclusions from this experiment.

Another transcription factor which is associated with $\mathrm{Bcl}-\mathrm{xL}$ gene expression is $\mathrm{NF} \kappa \mathrm{B}$. NFkB has been found to promote tumorigenesis, which is mediated by the protection of cancer cells from the induction of apoptosis [162]. Correspondingly, Wang et al. suggested that NFkB promotes resistance in breast cancer cells by induced gene expression of Bcl-xL and IAPs (inhibitor of apoptosis) [163]. Moreover, Huber et al. have shown that the IKK-2/I $\mathrm{B} \alpha / \mathrm{NF} \kappa \mathrm{B}$ pathway is required for the induction and maintenance of EMT in breast cancer. Thus, the inhibition of this pathway prevents EMT and in contrast, activation leads to a mesenchymal phenotype [164]. We hypothesize that EMT possibly results in activation of the NFкB signalling pathway, which subsequently induces the overexpression of Bcl-xL in the MSP RAS cells. In this context, it would be interesting to characterize the role of $\mathrm{NF \kappa B}$ in correlation to EMT and Bcl-xL gene expression in our model system. Consequently, the investigation of $\mathrm{NF \kappa B}$ activity as well as the inhibition of the respective factors could be helpful to elucidate the regulation of $\mathrm{Bcl}-\mathrm{xL}$ expression.

Additionally, concerning Bcl-xL regulation, recent reports defined the 5'UTR of Bcl-xL as a key regulatory element, which controls the translation of Bcl-xL mRNA [165]. Yoon et al. demonstrated that the 5'UTR of Bcl-xL mRNA contains an IRES (internal ribosome entry side) sequence, which is used in cells under stress [166]. Additionally, Durie et al. have shown that the RNA binding protein HuR (Hu antigen R) specifically binds to the 5 UTR of Bcl-xL, which results in the repression of Bcl-xL translation [165]. A separate assay would be required to examine translation in MSP RAS and $24^{+}$RAS cells. To this end, a polyribosomeanalyses assay would be useful to make a declaration about the efficiency of the mRNA translation in both cell lines.

Taken together, we manipulated several cellular molecules to investigate the effects on BclxL levels and on chemoresistance in MSP and MSP RAS cells. However, we were not able to 
confirm previous findings concerning $\mathrm{Bcl}-\mathrm{xL}$ regulation by miRNAs and specific transcription factors in our HMLE RAS system. We do not exclude the regulation of Bcl-xL or EMT by the addressed molecules, albeit we assume that missing effects could be due to the fact that we targeted modulatory mechanisms separately. The complexity of the EMT process suggests that reduction of Bcl-xL levels in MSP and MSP RAS cells probably needs a wide range of manipulating events, which in the end result in chemosensitive cells. Nevertheless, we successfully aimed to overcome the chemotherapeutic resistance in MSP RAS cells, using reagents that are already established in clinical trials.

\subsection{Targeting Bcl-xL in MSP RAS cells to overcome chemoresistance}

\subsubsection{Gossypol induces autophagy in HMLE RAS cells}

The overexpression of the anti-apoptotic Bcl-2 family members Bcl-2 and Bcl-xL have been found to contribute to metastasis and to interfere with the anti-tumor activity of chemotherapeutic drugs in cancer cells [155; 167]. It is possible that cancer cells develop a dependence on the protective effect of Bcl-2 and Bcl-xL for their survival [168]. Therefore, several studies pursued the development of anti-tumor agents that restore the apoptotic process. These antitumor agents sensitize resistant cancer cells to chemotherapy, by targeting the anti-apoptotic functions of Bcl-2 and Bcl-xL [167]. An inhibitor that specifically interacts with antiapoptotic Bcl-2 family members like Bcl-2 and Bcl-xL is Gossypol. This agent is a polyphenol, isolated from cotton seeds. Gossypol binds to the BH3-domain of anti-apoptotic proteins to neutralize their function [169]. Our aim was to examine whether Gossypol treatment sensitizes MSP RAS cells to chemotherapy. We have demonstrated that the application of Gossypol to HMLE RAS cells resulted in a proliferation stop in $24^{+}$RAS, as well as in MSP RAS cells (Figure 5.17A-C). In fact, we did not observe that Gossypol induces apoptosis in these cells, but rather autophagy (Figure 5.18). This observation was previously described by Gao et al., providing evidence that Gossypol induces autophagy followed by apoptotic cell death in MCF7 cells [135]. Mechanistically, they have shown that the BH3-only protein Beclin1, which is an inducer of autophagy, binds to the anti-apoptotic Bcl-2 family members. This interaction inhibits Beclin1 function. However, the dissociation of Beclin1 from its inhibitors triggers autophagy in the cell [135]. Gossypol is a BH3 mimetic and therefore able to bind the BH3-binding groove of Bcl-2 and Bcl-xL. The interaction between Gossypol and Bcl-2 or $\mathrm{Bcl}-\mathrm{xL}$ interrupts the binding of $\mathrm{Bcl}-2$ or $\mathrm{Bcl}-\mathrm{xL}$ to the pro-apoptotic $\mathrm{Bcl}-2$ family members Bax and Bim, which initiates apoptosis. But it also interrupts the binding of Bcl-2 or Bcl-xL 
to Beclin1, inducing autophagy. We wonder why we did not detect induced apoptosis, but rather autophagy in both $24^{+}$RAS and MSP RAS cells. Gao et al. hypothesized that, during the early stages of Gossypol treatment, the integrity of the mitochondria membranes is relatively stable. This results in autophagy as a protective mechanism in the cell, ensuring the turnover of damaged mitochondria. However, at later stages or at higher Gossypol dosages, mitochondria outer membrane permeabilization occurs and induces apoptosis. They suggested that autophagy delays the initiation of apoptosis, especially confirmed by the fact that the inhibition of autophagy immediately amplified the initiation of apoptosis by Gossypol [135]. In fact, we treated HMLE RAS cells for $16 \mathrm{~h}$ with $5 \mu \mathrm{M}$ and $10 \mu \mathrm{M}$ Gossypol and were unable to detect apoptosis induction. Even higher Gossypol concentrations, applied for the long-term proliferation assay, did not reveal typical apoptotic cell shapes of treated cells (Figure 5.17). For this reason, we doubt that this explanation applies to our cell system. Interestingly, Lian et al. suggested that Gossypol preferentially induces autophagy in apoptosis-resistant cells with high Bcl-2/Bcl-xL levels and apoptosis in cells with low Bcl-2/Bcl-xL levels [136]. This implies that the induced mode of cell death by Gossypol is dependent on the cellular context. Possibly, in cells containing low Bcl-2/Bcl-xL levels, the inhibition of the interaction with pro-apoptotic members of the Bcl-2 family is sufficient to shift the balance towards apoptosis. However, in cells with high levels of Bcl-2/Bcl-xL, the initiation of autophagy occurs more rapidly due to the inhibition of the interaction of Bcl-2/Bcl-xL with Beclin1 after Gossypol treatment [136]. The observation that Gossypol induces autophagy in MSP RAS and in $24^{+}$ RAS cells can be explained by the possibility that the Bcl-xL and Bcl-2 protein levels in $24^{+}$ RAS cells are probably high enough to prevent the induction of apoptosis after Gossypol treatment, rather than autophagy. To verify this idea, we propose to knock down Bcl-xL and possibly Bcl-2, followed by Gossypol treatment, to examine the induction of apoptosis. Additionally, it has been shown for another BH3 mimetic, ABT-737, that this reagent induces autophagy, as well [170]. It would be interesting to verify whether ABT-737 induces autophagy, but not apoptosis in the HMLE RAS system, too. Importantly, using reagents that target autophagy to kill cancer cells is a promising new strategy for cancer therapy. Even in our system, the application of Gossypol treatment was successful in inhibiting cell proliferation in chemoresistant MSP RAS cells, representing a potent reagent to overcome chemoresistance in these cells. In this context, Wolter et al. performed xenograft experiments with chemoresistant, high Bcl-xL-expressing head and neck squamous cell carcinoma (HNSCC). The authors have shown that the treatment of these tumors with Gossypol resulted in decreased mitotic rates and an increase in the number of apoptotic cells in comparison to the control mice 
[169]. To explore whether Gossypol affects MSP RAS cells under physiological conditions, we are going to perform xenograft experiments, treating MSP RAS cell-induced tumors with Gossypol.

\subsubsection{Bcl-xL destabilization mediated by HSP90 inhibition induced apoptosis in MSP RAS cells}

Besides targeting Bcl-xL in MSP RAS cells with Gossypol, we demonstrated that the destabilization of Bcl-xL mediated by HSP90 inhibition sensitized these cells to chemotherapy. Particularly, the inhibition of the HSP90 chaperone-function with the specific HSP90-inhibitors 17AAG and 17DMAG resulted in the induction of cleaved Caspase 3 and cleaved PARP in MSP RAS cells (Figure 5.19A). Caldas-Lopes et al. have previously shown that Bcl-xL is a direct client of HSP90 [137]. Correspondingly to our results, they have demonstrated that the inhibition of HSP90 with the inhibitor PU-H71 induced apoptosis in triple-negative Breast cancer cells, partly by the inactivation and down-regulation of the anti-apoptotic protein Bcl$\mathrm{xL}$, as well as the pro-survival protein Akt [137]. We observed reduced Akt protein levels in our experiments, as well. Interestingly, Akt inhibition alone did not induce apoptosis in MSP RAS cells, implying that Bcl-xL is essential for preventing apoptosis in our system (Figure 5.5A). This assumption was further confirmed by the fact that we were not able to induce apoptosis after 17AAG-mediated HSP90 inhibition in $24^{+\mathrm{Bcl}-\mathrm{xL}}$ and MSP ${ }^{\mathrm{Bcl}-\mathrm{xL}}$ cells, which continuously express high levels of Bcl-xL (Figure 5.19B). In addition, Nimmanapalli et al. have demonstrated that 17AAG treatment resulted in destabilization of the growth- and survival-signalling proteins c-Raf-1, Akt and c-Src; and additionally, in the conformational change of the pro-apoptotic protein Bax [171]. However, when they overexpressed Bcl-xL in human leukemic cells (HL-60) and human breast cancer cells (MDA-MB-468), they also failed to induce apoptosis after 17AAG treatment. In fact, this could not be explained by the failed destabilization of further HSP90 client proteins, which were still decreased upon HSP90 inhibition, but rather by the lack of mitochondrial events of apoptosis, supporting our

findings in $24^{+\mathrm{Bcl}-\mathrm{xL}}$ and MSP ${ }^{\mathrm{Bcl}-\mathrm{xL}}$ cells [171]. These results indicate that Bcl-xL stabilization in MSP RAS cells is in part mediated by HSP90, confirmed by the marked decrease in Bcl-xL protein levels after HSP90 inhibition, which contributed to apoptosis in MSP RAS cells and the failed induction of apoptosis in Bcl-xL overexpressing HMLE cells.

Further, it has been shown for mammary tumors that they contain increased levels of antiapoptotic Bcl-2 family members, as well as increased levels of the heat shock proteins HSP70 
and HSP90 [172]. It is suggested that HSP70 and HSP90 are able to inhibit the apoptotic cascade by preventing the formation of the apoptosome or induction of Fas signalling, which contributes to the formation of malignant cells $[173 ; 174 ; 175]$. In contrast to these findings, we did not detect increased HSP90 levels in MSP RAS compared to $24^{+}$RAS cells, implying that HSP90 is not executing these functions in MSP RAS cells to prevent apoptosis (data not shown). However, considering the high number of HSP90 clients which are stabilized by HSP90, we assume that not only HSP90-mediated Bcl-xL stabilization plays a role for the induction of apoptosis in MSP RAS cells after HSP90 inhibition. Rather, the destabilization of transcription factors, such as STAT3 and STAT5, which are involved in Bcl-xL gene expression, or EMT-regulating factors, like $\mathrm{NF \kappa B}$, which possibly maintain the mesenchymal state of the MSP RAS cells, may be important for the induction of apoptosis after abolishing HSP90 chaperone function [176; 177]. Possibly, the inhibition of EMT-inducing molecules and Bcl-xL transcription factors, mediated by HSP90 inhibition, is necessary to induce a decrease in Bcl-xL levels in MSP RAS cells and subsequently apoptosis.

17AAG and 17DMAG are promising anti-tumor agents, which are already used in clinical trials $[178 ; 179]$. We performed one preliminary xenograft experiment, treating MSP RAS cellinduced tumors with 17DMAG (Figure 5.21). We have demonstrated that in comparison to the control group 17DMAG treatment resulted in reduced tumor sizes. 17DMAG displays less hepatotoxicity compared to $17 \mathrm{AAG}$ and is easily soluble in water [180; 181]. In addition, in vitro clonogenic assays revealed lower $\mathrm{IC}_{50}$ for $17 \mathrm{DMAG}$ than for $17 \mathrm{AAG}$, implicating that 17DMAG is a more potent anti-tumor drug [182; 183]. The efficacy of 17DMAG has been verified in several xenograft models, including MDA-MB-231 (breast cancer) and NCI-H522 (adenocarcinoma). Clinical phase I studies have shown that HSP90 inhibition in Her-2 positive breast cancer cells combined with Trastuzumab, a cytostatic agent used for breast cancer, is well tolerated and inhibits tumor growth in vivo [184]. Our first result indicates that we are able to overcome the resistant phenotype of MSP RAS cells in vivo by inhibiting HSP90 in mice. Considering the characteristics of MSP RAS cells, such as being cells that have undergone EMT, containing stem cell like properties, being highly invasive and forming metastasis, we are now able to target such cells, resulting in inhibited tumor growth in vivo and chemosensitivity in vitro. 


\subsubsection{The role of Salinomycin in treating cancer stem cells}

Another agent that is able to specifically eliminate cancer stem cells (CSC), an attribute that is associated with our MSP RAS cells as well, is Salinomycin. Salinomycin was found by performing a high throughput screen to identify selective inhibitors for CSC [185]. Salinomycin is a ionophore antibiotic with the ability to kill CSC as well as chemoresistant tumor cells [186]. Indeed, by the performance of long-term-survival assays, we have shown that MSP RAS cells are more sensitive to Salinomycin than $24^{+}$RAS cells (Figure 8.1, appendix). Hitherto, Salinomycin was only used in veterinary medicine. However, Gupta et al. showed in previous studies that Salinomycin is 100 times more efficient in killing breast cancer stem cell like cells than Taxol [185]. Interestingly, Gupta et al. used human breast cancer cell lines for their investigations, which have been experimentally induced to undergo EMT. Particularly, they worked with HMLE and HMLE RAS cells and induced EMT on the one hand via shRNA-mediated gene silencing of E-Cadherin and on the other hand via the overexpression of the EMT-transcription factor Twist. Both experimental settings resulted in cells that have undergone EMT associated with a mesenchymal phenotype. Nevertheless, upon EMT cells displayed resistance towards the chemotherapeutic drugs Paclitaxel ( 20 fold increase in $\left.\mathrm{IC}_{50}\right)$ and Doxorubicin ( $\sim 5$ fold increase) compared to the respective HMLE and HMLE RAS controls [185]. In fact, consistent with our results, the treatment with Salinomycin decreased the proportion of $\mathrm{CD}_{4} 4^{+} / \mathrm{CD} 24^{-}$cells by 20 -fold in comparison to the controls and additionally, decreased the number of tumor-spheres by 10-fold [185]. Despite these impressive findings, an important caveat for the clinical use of Salinomycin is its toxicity [186]. It is known that Salinomycin exhibits toxicity towards neuronal cells and additionally reduces cell viability [187]. An important fact is that Salinomycin can only be applied by intravenous injection, because the oral application of Saliomycin results in life-endangering rhabdomyolysis [188]. This particular disadvantage of Salinomycin should be considered when establishing this antibiotic as an anti-cancer reagent. In contrast, the HSP90 inhibitors 17AAG and 17DMAG, which work efficiently in our EMT system to eliminate MSP RAS cells, are well established within clinical trials and do not show any detrimental activity. 


\subsection{Relevance of our findings in human cancer diseases and future aspects}

\subsubsection{Migrating breast cancer cells combine the attributes of EMT and Bcl-xL overex- pression}

The presence of EMT in human cancer has been intensely discussed during the last decades. Findings in pre-clinical models suggest that at least a partial EMT occurs during cancer progression, supporting tumor metastasis and chemoresistance [93]. Within our studies, we have demonstrated that EMT renders HMLE as well as HMLE RAS cells resistant to chemotherapy and particularly resistant to apoptosis induction mediated by the overexpression of Bcl-xL. Correspondingly, we made use of immunohistochemical approaches to characterize $\mathrm{Bcl}-\mathrm{xL}$ protein levels in human breast cancer tissues (Figure 5.11A-C). We found increased Bcl-xL protein levels at the stroma interface and in disseminated tumor cells. Staining for Cytokeratin 8 indicated that the disseminated tumor cells belong to the tumor, but not to stroma (Figure 5.11D). In this context, it has been previously shown that the dissemination of tumor cells is a prerequisite to form metastasis associated with EMT at the invasive front $[93 ; 94 ; 189]$. Not only is the autonomous activation of EMT-relevant pathways required, but rather costimulatory signals from the microenvironment at the tumor-stroma interface are necessary to induce EMT [189]. Additionally, the accumulation of genetic alterations occurs in these cells, providing them with the ability to survive in distant tissues [189]. In fact, Brabletz et al. suggested the presence of migrating cancer stem (MCS) cells at the invasive front, combining the attributes of stemness and mobility mediated by EMT [189]. They assumed that breast cancer meets both of these attributes of the MCS-cell concept, which explains tumor metastasis [189]. Consistently, very recent findings by Yu et al. characterized EMT in circulating tumors cells (CTC) from human breast cancer tissues [190]. They found that mesenchymal cells are highly enriched in CTCs and proposed an association of mesenchymal CTCs and disease progression. In particular, the comparison of CTCs in pre- and post-treatment blood samples revealed that progressive disease is associated with increased numbers of mesenchymal CTCs [190]. In addition, they defined active EMT-related signalling pathways, including the TGF $\beta$ and Wnt-signalling pathways, and the EMT-inducing transcription factor Forkhead box protein $\mathrm{C} 1$ (FOXC1) in these cells. These results indicate the presence of EMT in human breast cancer samples [190]. Data from these previous studies correlate well with our findings. Disseminated cancer cells appear to be associated with EMT and the overexpression of Bcl-xL. Thus, an important step to define the correlation between EMT and the overexpression of Bcl- 
$\mathrm{xL}$ in breast cancer is to prove the presence of EMT-associated transcription factors, such as Twist or Zeb1/2, in the disseminated cells from our breast cancer tissue samples.

\subsubsection{Chromosomal rearrangements lead to Bcl-xL overexpression in cancer and em- bryonic stem cells}

In general, the overexpression of Bcl-xL has not only been detected in breast cancer, but also in prostate, colon and pancreatic cancer, as well as multiple myeloma and melanoma [76; 157]. Our findings suggest that chemoresistance acquired during the process of EMT, even in stem cell like cancer cells, is accounted for by the overexpression of the anti-apoptotic protein Bcl-xL. Bai et al. found ectopic expression of Bcl-xL in human embryonic stem cells (hESC) which resulted in a significant increase of hESC colonies in single-cell suspension cultures [151]. This very recent report showed that the overexpression of Bcl-xL in hESCs resulted in attenuation of apoptosis, increased efficiency to generate embryoid bodies in vitro and formation of teratoma by dissociated hESCs in vivo. Consistent with these data, findings from the initial stem cell initiative revealed that human embryonic stem (ES) cells acquired karyotypically changes on prolonged cell culture [191]. In particular, a minimal amplicon in chromosome 20q11.21 occurred in $<20 \%$ of the investigated ES cell lines. The amplicon includes three genes expressed in human ES cells, one of these genes is Bcl2L1 (Bcl-xL is an isoform of this gene) [191]. Accordingly, a genome-wide RNAi screen exhibited a prominent role of Bc12L1 in human ES biology [192]. Strikingly, a recent structural variant screen of $>3000$ samples from 24 cancer cell types identified a reoccurring gain in 20q11.21, including Bcl2L1 within the minimal amplicon, as well [191; 193]. In conclusion, these observations imply that similar mutations occur in ES and cancer cells, resulting in the overexpression of Bcl-xL. The similarity of these mutations suggests equal underlying mechanisms [191]. This raised the idea that similar alterations might be present in cells upon EMT, resulting in Bcl-xL overexpression. In this context it is of particular interest to analyze the karyotypes of MSP RAS compared to $24^{+}$RAS cells. These results would give an idea, as to what extent chromosomal rearrangements contribute to overexpression of Bcl-xL in MSP RAS cells, leading to chemoresistance.

\subsubsection{Gossypol and HSP90 inhibitors as new strategies to eliminate chemoresistant cells in human cancer therapies}

Finally, we aimed to find an option to overcome chemoresistance in MSP RAS cells. Antiapoptotic proteins are an attractive target for the development of anti-cancer agents, given the fact that high levels of anti-apoptotic Bcl-2 family members are associated with chemo- 
resistance [194]. Thus, Fiebig et al. have shown that the overexpression of Bcl-xL in MCF7 cells is ten times more efficient to render cells chemoresistant than Bcl-2 [81]. We demonstrated in vitro and in vivo that the BH3 mimetic Gossypol and HSP90 inhibition, respectively, sensitized MSP RAS cells and induced cell death (Figure 5.17C, Figure 5.19A, Figure 5.21). As mentioned before, both reagents are already established in clinical trials and possibly usable in combinations with conventional therapy to treat cancer. On the one hand, 17AAG-mediated HSP90 inhibition combined with Trastuzumab was a convincing possibility for the treatment of breast cancer patients that are resistant to Trastuzumab alone [195]. Clinical studies revealed mild to moderate toxicities and suggested that HSP90 inhibition in vivo is sufficient to cause inhibition of tumor growth [195]. The combination of HSP90 inhibitors with chemotherapeutic agents was demonstrated to be additive, as it was shown for 17AAG and Carboplatin in ovarian cancer cells. Further, the application of 17AAG in combination with Taxol or Doxorubicin in breast cancer cell lines was synergistic, displaying a greater advantage of 17AAG to treat cancer [196; 197]. On the other hand, Gossypol was tested in several clinical trials in which cancer patients were treated with Gossypol in combination with conventional therapy. Recent clinical studies investigated the treatment of small cell lung cancer and solid tumors with Gossypol in combination with Cisplatin or Etoposide [198]. Further, treatments of metastatic cancer and cancers that cannot be removed by surgery were performed using Gossypol in combination with Paclitaxel or Carboplatin [199]. Additionally, previous studies in melanoma, colon and breast cancer have demonstrated that Gossypol is well tolerated and contributed to reduced tumor volume $[200 ; 201 ; 202 ; 203]$, indicating that Gossypol might be applicable for a wide range of cancer types and thus represents a promising agent for cancer therapy [204]. These results indicate that both reagents, 17DMAG and Gossypol, may be considered as anti-cancer drugs for tumor therapies. The fact that both chemotherapeutics are able to induce cell death in MSP RAS cells suggests that both reagents probably contain the competency to eliminate resistant cells in vivo.

\subsection{Summary}

The data presented here demonstrate that EMT can render cells chemoresistant. We defined the anti-apoptotic protein $\mathrm{Bcl}-\mathrm{xL}$ as a key factor required for chemoresistance, which is overexpressed in cells that have undergone EMT. si-RNA-mediated knock down of Bcl-xL made MSP RAS cells chemosensitive, highlighting Bcl-xL as a promising drug target to sensitize cancer cells to chemotherapy. Additionally, our first xenograft experiment revealed HSP90 inhibitors as possible anti-tumor agents for stemness-associated, metastatic cancer cells. 
The comparison of our findings to those from previous studies implies a correlation between EMT, chemoresistance, cancer stem cell features and Bcl-xL overexpression. Nevertheless, further studies are required to define the precise mechanism behind Bcl-xL up-regulation upon EMT. However, this report describes Bcl-xL as a key mediator of apoptosis-resistance in cells upon EMT and as a determinant of life and death in chemotherapy. 


\section{References}

[1]J. Ferlay, E. Steliarova-Foucher, J. Lortet-Tieulent, S. Rosso, J.W. Coebergh, H. Comber, D. Forman, F. Bray, Cancer incidence and mortality patterns in Europe: estimates for 40 countries in 2012. Eur J Cancer 49 (2013) 1374-1403.

[2]H.D. Suit, Local control and patient survival. International journal of radiation oncology, biology, physics 23 (1992) 653-660.

[3]J. Sleeman, P.S. Steeg, Cancer metastasis as a therapeutic target. European Journal of Cancer 46 (2010) 1177-1180.

[4]X. Li, M.T. Lewis, J. Huang, C. Gutierrez, C.K. Osborne, M.F. Wu, S.G. Hilsenbeck, A. Pavlick, X. Zhang, G.C. Chamness, H. Wong, J. Rosen, J.C. Chang, Intrinsic resistance of tumorigenic breast cancer cells to chemotherapy. Journal of the National Cancer Institute 100 (2008) 672-679.

[5]P.S. Steeg, D. Theodorescu, Metastasis: a therapeutic target for cancer. Nature clinical practice. Oncology 5 (2008) 206-219.

[6]K.F. Aktories, U.; Hofmann, B.; Starke K., Allgemeine und spezielle Pharmakologie und Toxikologie, Elsevier, Urban \& Fischer, 2008.

[7]T. Helleday, E. Petermann, C. Lundin, B. Hodgson, R.A. Sharma, DNA repair pathways as targets for cancer therapy. Nat Rev Cancer 8 (2008) 193-204.

[8]Y. Pommier, R.B. Diasio, (Eds.) Pharmacological and Therapeutic agents that target DNA Replication, Cold Spring Harbor Press, 2006.

[9]G. Minotti, P. Menna, E. Salvatorelli, G. Cairo, L. Gianni, Anthracyclines: molecular advances and pharmacologic developments in antitumor activity and cardiotoxicity. Pharmacological reviews 56 (2004) 185-229.

[10]P. D'Arpa, L.F. Liu, Topoisomerase-targeting antitumor drugs. Biochimica et biophysica acta 989 (1989) 163-177.

[11]N.R. Bachur, F. Yu, R. Johnson, R. Hickey, Y. Wu, L. Malkas, Helicase inhibition by anthracycline anticancer agents. Molecular pharmacology 41 (1992) 993-998.

[12]P.J. Loehrer, L.H. Einhorn, Drugs five years later. Cisplatin. Annals of internal medicine 100 (1984) 704-713.

[13]B. Rosenberg, L. VanCamp, J.E. Trosko, V.H. Mansour, Platinum compounds: a new class of potent antitumour agents. Nature 222 (1969) 385-386.

[14]B. Liedert, D. Pluim, J. Schellens, J. Thomale, Adduct-specific monoclonal antibodies for the measurement of cisplatin-induced DNA lesions in individual cell nuclei. Nucleic acids research 34 (2006) e47.

[15]M. Kartalou, J.M. Essigmann, Mechanisms of resistance to cisplatin. Mutation research 478 (2001) 23-43.

[16]C.M. Sorenson, M.A. Barry, A. Eastman, Analysis of events associated with cell cycle arrest at G2 phase and cell death induced by cisplatin. Journal of the National Cancer Institute 82 (1990) 749-755.

[17]R.A. Alderden, M.D. Hall, T.W. Hambley, The discovery and development of cisplatin. J Chem Educ 83 (2006) 728-734.

[18]L. Kelland, The resurgence of platinum-based cancer chemotherapy. Nat Rev Cancer 7 (2007) 573-584.

[19]E. Wong, C.M. Giandomenico, Current status of platinum-based antitumor drugs. Chemical reviews 99 (1999) 2451-2466.

[20]K. Kolaric, D. Vukas, Carboplatin activity in untreated metastatic breast cancer patients-results of a phase II study. Cancer chemotherapy and pharmacology 27 (1991) 409412. 
[21]M. Martin, E. Diaz-Rubio, A. Casado, P. Santabarbara, J.M. Lopez Vega, E. Adrover, L. Lenaz, Carboplatin: an active drug in metastatic breast cancer. J Clin Oncol 10 (1992) 433-437.

[22]G. Fountzilas, A.M. Dimopoulos, C. Papadimitriou, A. Kalogera-Fountzila, G. Aravantinos, D. Bafaloukos, A. Athanassiades, C. Nicolaides, A. Keramopoulos, N. Pavlidis, P. Kosmidis, D. Skarlos, First-line chemotherapy with paclitaxel by threehour infusion and carboplatin in advanced breast cancer (final report): a phase II study conducted by the Hellenic Cooperative Oncology Group. Annals of oncology : official journal of the European Society for Medical Oncology / ESMO 9 (1998) 1031-1034.

[23]E.A. Perez, D.W. Hillman, P.J. Stella, J.E. Krook, L.C. Hartmann, T.R. Fitch, A.K. Hatfield, J.A. Mailliard, S. Nair, C.G. Kardinal, J.N. Ingle, A phase II study of paclitaxel plus carboplatin as first-line chemotherapy for women with metastatic breast carcinoma. Cancer 88 (2000) 124-131.

[24]G. Fountzilas, A. Athanassiadis, A. Kalogera-Fountzila, G. Aravantinos, D. Bafaloukos, E. Briasoulis, N. Dombros, I. Ioannidis, N. Pavlidis, P. Kosmidis, D. Skarlos, Paclitaxel by 3-h infusion and carboplatin in anthracycline-resistant advanced breast cancer. A phase II study conducted by the Hellenic Cooperative Oncology Group. Eur J Cancer 33 (1997) 1893-1895.

[25]M.D. Pegram, T. Pienkowski, D.W. Northfelt, W. Eiermann, R. Patel, P. Fumoleau, E. Quan, J. Crown, D. Toppmeyer, M. Smylie, A. Riva, S. Blitz, M.F. Press, D. Reese, M.A. Lindsay, D.J. Slamon, Results of two open-label, multicenter phase II studies of docetaxel, platinum salts, and trastuzumab in HER2-positive advanced breast cancer. Journal of the National Cancer Institute 96 (2004) 759-769.

[26]H. Brockmann, [Anthracyclinones and Anthracyclines. (Rhodomycinone, Pyrromycinone and Their Glycosides)]. Fortschritte der Chemie organischer Naturstoffe = Progress in the chemistry of organic natural products. Progres dans la chimie des substances organiques naturelles 21 (1963) 121-182.

[27]D.A. Gewirtz, A critical evaluation of the mechanisms of action proposed for the antitumor effects of the anthracycline antibiotics adriamycin and daunorubicin. Biochemical pharmacology 57 (1999) 727-741.

[28]N. Ishida, K. Miyazaki, K. Kumagai, M. Rikimaru, Neocarzinostatin, an Antitumor Antibiotic of High Molecular Weight. Isolation, Physiochemical Properties and Biological Activities. The Journal of antibiotics 18 (1965) 68-76.

[29]J. Meienhofer, H. Maeda, C.B. Glaser, J. Czombos, K. Kuromizu, Primary structure of neocarzinostatin, an antitumor protein. Science 178 (1972) 875-876.

[30]A. Galat, I.H. Goldberg, Molecular models of neocarzinostatin damage of DNA: analysis of sequence dependence in 5'GAGCG:5'CGCTC. Nucleic acids research 18 (1990) 2093-2099.

[31]I.H. Goldberg, Mechanism of Neocarcinostatin Action: Role of DNA Microstructure in Determination of Chemistry of Biostranded Oxidative Damage. Accounts of Chemical Research 24 (1991) 8.

[32]S.S. Legha, D.D. Von Hoff, M. Rozencweig, D. Abraham, M. Slavik, M. Muggia, Neocarzinostatin (NSC 157365) a new cancerostatic compound. Oncology 33 (1976) 265-270.

[33]G. Falkson, D. Von Hoff, D. Klaassen, H. Du Plessis, C.F. Van Der Merwe, A.M. Van Der Merwe, P.P. Carbone, A phase II study of neocarzinostatin (NSC 157365) in malignant hepatoma. An Eastern Cooperative Oncology Group pilot study. Cancer chemotherapy and pharmacology 4 (1980) 33-36.

[34]D.B. Longley, P.G. Johnston, Molecular mechanisms of drug resistance. The Journal of pathology 205 (2005) 275-292. 
[35]L.N. Abdullah, E.K. Chow, Mechanisms of chemoresistance in cancer stem cells. Clinical and translational medicine 2 (2013) 3.

[36]M.M. Gottesman, Mechanisms of cancer drug resistance. Annu Rev Med 53 (2002) 615627.

[37]M. Komatsu, T. Sumizawa, M. Mutoh, Z.S. Chen, K. Terada, T. Furukawa, X.L. Yang, H. Gao, N. Miura, T. Sugiyama, S. Akiyama, Copper-transporting P-type adenosine triphosphatase (ATP7B) is associated with cisplatin resistance. Cancer research 60 (2000) 1312-1316.

[38]C.A. Rabik, M.E. Dolan, Molecular mechanisms of resistance and toxicity associated with platinating agents. Cancer treatment reviews 33 (2007) 9-23.

[39]K. Hamaguchi, A.K. Godwin, M. Yakushiji, P.J. O'Dwyer, R.F. Ozols, T.C. Hamilton, Cross-resistance to diverse drugs is associated with primary cisplatin resistance in ovarian cancer cell lines. Cancer research 53 (1993) 5225-5232.

[40]Y. Xu, M.A. Villalona-Calero, Irinotecan: mechanisms of tumor resistance and novel strategies for modulating its activity. Annals of oncology : official journal of the European Society for Medical Oncology / ESMO 13 (2002) 1841-1851.

[41]H. Lage, H. Helmbach, M. Dietel, D. Schadendorf, Modulation of DNA topoisomerase II activity and expression in melanoma cells with acquired drug resistance. British journal of cancer 82 (2000) 488-491.

[42]G.J. Peters, H.H. Backus, S. Freemantle, B. van Triest, G. Codacci-Pisanelli, C.L. van der Wilt, K. Smid, J. Lunec, A.H. Calvert, S. Marsh, H.L. McLeod, E. Bloemena, S. Meijer, G. Jansen, C.J. van Groeningen, H.M. Pinedo, Induction of thymidylate synthase as a 5-fluorouracil resistance mechanism. Biochimica et biophysica acta 1587 (2002) 194-205.

[43]S.W. Johnson, P.B. Laub, J.S. Beesley, R.F. Ozols, T.C. Hamilton, Increased platinumDNA damage tolerance is associated with cisplatin resistance and cross-resistance to various chemotherapeutic agents in unrelated human ovarian cancer cell lines. Cancer research 57 (1997) 850-856.

[44]E.L. Mamenta, E.E. Poma, W.K. Kaufmann, D.A. Delmastro, H.L. Grady, S.G. Chaney, Enhanced replicative bypass of platinum-DNA adducts in cisplatin-resistant human ovarian carcinoma cell lines. Cancer research 54 (1994) 3500-3505.

[45]F. Bunz, Cell death and cancer therapy. Current opinion in pharmacology 1 (2001) 337341.

[46]J.C. Reed, T. Miyashita, S. Takayama, H.G. Wang, T. Sato, S. Krajewski, C. AimeSempe, S. Bodrug, S. Kitada, M. Hanada, BCL-2 family proteins: regulators of cell death involved in the pathogenesis of cancer and resistance to therapy. Journal of cellular biochemistry 60 (1996) 23-32.

[47]T.R. Wilson, D.B. Longley, P.G. Johnston, Chemoresistance in solid tumours. Annals of oncology : official journal of the European Society for Medical Oncology / ESMO 17 Suppl 10 (2006) x315-324.

[48]D.N. Dhanasekaran, E.P. Reddy, JNK signaling in apoptosis. Oncogene 27 (2008) 62456251.

[49]G. Gebauer, B. Mirakhur, Q. Nguyen, S.K. Shore, H. Simpkins, N. Dhanasekaran, Cisplatin-resistance involves the defective processing of MEKK1 in human ovarian adenocarcinoma 2008/C13 cells. International journal of oncology 16 (2000) 321-325.

[50]P. Blume-Jensen, T. Hunter, Oncogenic kinase signalling. Nature 411 (2001) 355-365.

[51]S. Elmore, Apoptosis: a review of programmed cell death. Toxicologic pathology 35 (2007) 495-516.

[52]J.F. Kerr, A.H. Wyllie, A.R. Currie, Apoptosis: a basic biological phenomenon with wide-ranging implications in tissue kinetics. British journal of cancer 26 (1972) 239257. 
[53]A. Lawen, Apoptosis-an introduction. BioEssays : news and reviews in molecular, cellular and developmental biology 25 (2003) 888-896.

[54]A. Gewies, Indroduction to Apoptosis, in, ApoReview, http://www.celldeath.de/encyclo/aporev/aporev.htm, 2003.

[55]S.M. Nelson, L.R. Ferguson, W.A. Denny, DNA and the chromosome - varied targets for chemotherapy. Cell \& chromosome 3 (2004) 2.

[56]M.S. Satoh, T. Lindahl, Role of poly(ADP-ribose) formation in DNA repair. Nature 356 (1992) 356-358.

[57]I. Bohm, [The apoptosis marker enzyme poly-(ADP-ribose) polymerase (PARP) in systemic lupus erythematosus]. Zeitschrift fur Rheumatologie 65 (2006) 541-544.

[58]K. Lauber, Produktion von monozytären Attraktionssignalen während der Apoptose: Charakterisierung eines neuen Aspekts bei der Eliminierung apoptotischer Zellen, in, Institue for Medical Virology, University of Tübingen, 2003.

[59]C. Teixeira, J.C. Reed, M.A. Pratt, Estrogen promotes chemotherapeutic drug resistance by a mechanism involving Bcl-2 proto-oncogene expression in human breast cancer cells. Cancer research 55 (1995) 3902-3907.

[60]M. Kandouz, M. Siromachkova, D. Jacob, B. Chretien Marquet, A. Therwath, A. Gompel, Antagonism between estradiol and progestin on Bcl-2 expression in breastcancer cells. International journal of cancer. Journal international du cancer 68 (1996) 120-125.

[61]S.L. Sankari, K.M. Masthan, N.A. Babu, T. Bhattacharjee, M. Elumalai, Apoptosis in cancer--an update. Asian Pacific journal of cancer prevention : APJCP 13 (2012) 4873-4878.

[62]R.P. Rastogi, R.P. Sinha, Apoptosis: Molecular Mechanisms and Pathogenicity. EXCLI Journal 8 (2009) 155-181.

[63]A. Ashkenazi, Targeting death and decoy receptors of the tumour-necrosis factor superfamily. Nat Rev Cancer 2 (2002) 420-430.

[64]C. Scaffidi, S. Fulda, A. Srinivasan, C. Friesen, F. Li, K.J. Tomaselli, K.M. Debatin, P.H. Krammer, M.E. Peter, Two CD95 (APO-1/Fas) signaling pathways. The EMBO journal 17 (1998) 1675-1687.

[65]M. Crompton, Bax, Bid and the permeabilization of the mitochondrial outer membrane in apoptosis. Current opinion in cell biology 12 (2000) 414-419.

[66]A. Gross, J.M. McDonnell, S.J. Korsmeyer, BCL-2 family members and the mitochondria in apoptosis. Genes \& development 13 (1999) 1899-1911.

[67]K.C. Zimmermann, C. Bonzon, D.R. Green, The machinery of programmed cell death. Pharmacology \& therapeutics 92 (2001) 57-70.

[68]R.J. Youle, A. Strasser, The BCL-2 protein family: opposing activities that mediate cell death. Nature reviews. Molecular cell biology 9 (2008) 47-59.

[69]S. Cory, Regulation of lymphocyte survival by the bcl-2 gene family. Annual review of immunology 13 (1995) 513-543.

[70]C. Schwerk, K. Schulze-Osthoff, Regulation of apoptosis by alternative pre-mRNA splicing. Molecular cell 19 (2005) 1-13.

[71]N. Motoyama, F. Wang, K.A. Roth, H. Sawa, K. Nakayama, I. Negishi, S. Senju, Q. Zhang, S. Fujii, et al., Massive cell death of immature hematopoietic cells and neurons in Bcl-x-deficient mice. Science 267 (1995) 1506-1510.

[72]L. Sevilla, A. Zaldumbide, P. Pognonec, K.E. Boulukos, Transcriptional regulation of the bcl-x gene encoding the anti-apoptotic Bcl-xL protein by Ets, Rel/NFkappaB, STAT and AP1 transcription factor families. Histology and histopathology 16 (2001) 595601. 
[73]D.A. Grillot, M. Gonzalez-Garcia, D. Ekhterae, L. Duan, N. Inohara, S. Ohta, M.F. Seldin, G. Nunez, Genomic organization, promoter region analysis, and chromosome localization of the mouse bcl-x gene. J Immunol 158 (1997) 4750-4757.

[74]R. Eferl, E.F. Wagner, AP-1: A double-edged sword in tumorigenesis. Nat Rev Cancer 3 (2003) 859-868.

[75]L. Sevilla, C. Aperlo, V. Dulic, J.C. Chambard, C. Boutonnet, O. Pasquier, P. Pognonec, K.E. Boulukos, The Ets2 transcription factor inhibits apoptosis induced by colonystimulating factor 1 deprivation of macrophages through a Bcl-xL-dependent mechanism. Molecular and cellular biology 19 (1999) 2624-2634.

[76]J.M. Grad, X.R. Zeng, L.H. Boise, Regulation of Bcl-xL: a little bit of this and a little bit of STAT. Current opinion in oncology 12 (2000) 543-549.

[77]J.I. Song, J.R. Grandis, STAT signaling in head and neck cancer. Oncogene 19 (2000) 2489-2495.

[78]M. Horita, E.J. Andreu, A. Benito, C. Arbona, C. Sanz, I. Benet, F. Prosper, J.L. Fernandez-Luna, Blockade of the Bcr-Abl kinase activity induces apoptosis of chronic myelogenous leukemia cells by suppressing signal transducer and activator of transcription 5-dependent expression of Bcl-xL. The Journal of experimental medicine 191 (2000) 977-984.

[79]S. Shimizu, T. Takehara, H. Hikita, T. Kodama, T. Miyagi, A. Hosui, T. Tatsumi, H. Ishida, T. Noda, H. Nagano, Y. Doki, M. Mori, N. Hayashi, The let-7 family of microRNAs inhibits Bcl-xL expression and potentiates sorafenib-induced apoptosis in human hepatocellular carcinoma. Journal of hepatology 52 (2010) 698-704.

[80]Y. Fernandez, L. Espana, S. Manas, A. Fabra, A. Sierra, Bcl-xL promotes metastasis of breast cancer cells by induction of cytokines resistance. Cell death and differentiation 7 (2000) 350-359.

[81]A.A. Fiebig, W. Zhu, C. Hollerbach, B. Leber, D.W. Andrews, Bcl-XL is qualitatively different from and ten times more effective than Bcl-2 when expressed in a breast cancer cell line. BMC cancer 6 (2006) 213.

[82]J. Yang, R.A. Weinberg, Epithelial-mesenchymal transition: at the crossroads of development and tumor metastasis. Dev Cell 14 (2008) 818-829.

[83]K. Polyak, R.A. Weinberg, Transitions between epithelial and mesenchymal states: acquisition of malignant and stem cell traits. Nat Rev Cancer 9 (2009) 265-273.

[84]L. Larue, A. Bellacosa, Epithelial-mesenchymal transition in development and cancer: role of phosphatidylinositol 3' kinase/AKT pathways. Oncogene 24 (2005) 7443-7454.

[85]J.P. Thiery, H. Acloque, R.Y. Huang, M.A. Nieto, Epithelial-mesenchymal transitions in development and disease. Cell 139 (2009) 871-890.

[86]P.A. Gregory, A.G. Bert, E.L. Paterson, S.C. Barry, A. Tsykin, G. Farshid, M.A. Vadas, Y. Khew-Goodall, G.J. Goodall, The miR-200 family and miR-205 regulate epithelial to mesenchymal transition by targeting ZEB1 and SIP1. Nature cell biology 10 (2008) 593-601.

[87]S. Brabletz, T. Brabletz, The ZEB/miR-200 feedback loop--a motor of cellular plasticity in development and cancer? EMBO reports 11 (2010) 670-677.

[88]P.A. Gregory, C.P. Bracken, A.G. Bert, G.J. Goodall, MicroRNAs as regulators of epithelial-mesenchymal transition. Cell Cycle 7 (2008) 3112-3118.

[89]U. Burk, J. Schubert, U. Wellner, O. Schmalhofer, E. Vincan, S. Spaderna, T. Brabletz, A reciprocal repression between ZEB1 and members of the miR-200 family promotes EMT and invasion in cancer cells. EMBO reports 9 (2008) 582-589.

[90]E.M. Teague, C.G. Print, M.L. Hull, The role of microRNAs in endometriosis and associated reproductive conditions. Human reproduction update 16 (2010) 142-165.

[91]C. Scheel, R.A. Weinberg, Cancer stem cells and epithelial-mesenchymal transition: concepts and molecular links. Semin Cancer Biol 22 (2012) 396-403. 
[92]G. Christofori, New signals from the invasive front. Nature 441 (2006) 444-450.

[93]A.G. de Herreros, S. Peiro, M. Nassour, P. Savagner, Snail family regulation and epithelial mesenchymal transitions in breast cancer progression. Journal of mammary gland biology and neoplasia 15 (2010) 135-147.

[94]I. Roxanis, Occurrence and significance of epithelial-mesenchymal transition in breast cancer. Journal of clinical pathology 66 (2013) 517-521.

[95]I.K. Guttilla, B.D. Adams, B.A. White, ERalpha, microRNAs, and the epithelialmesenchymal transition in breast cancer. Trends in endocrinology and metabolism: TEM 23 (2012) 73-82.

[96]A.J. Trimboli, K. Fukino, A. de Bruin, G. Wei, L. Shen, S.M. Tanner, N. Creasap, T.J. Rosol, M.L. Robinson, C. Eng, M.C. Ostrowski, G. Leone, Direct evidence for epithelial-mesenchymal transitions in breast cancer. Cancer research 68 (2008) 937945.

[97]J. Yang, S.A. Mani, J.L. Donaher, S. Ramaswamy, R.A. Itzykson, C. Come, P. Savagner, I. Gitelman, A. Richardson, R.A. Weinberg, Twist, a master regulator of morphogenesis, plays an essential role in tumor metastasis. Cell 117 (2004) 927-939.

[98]M. Al-Hajj, M.S. Wicha, A. Benito-Hernandez, S.J. Morrison, M.F. Clarke, Prospective identification of tumorigenic breast cancer cells. Proceedings of the National Academy of Sciences of the United States of America 100 (2003) 3983-3988.

[99]M. Shipitsin, L.L. Campbell, P. Argani, S. Weremowicz, N. Bloushtain-Qimron, J. Yao, T. Nikolskaya, T. Serebryiskaya, R. Beroukhim, M. Hu, M.K. Halushka, S. Sukumar, L.M. Parker, K.S. Anderson, L.N. Harris, J.E. Garber, A.L. Richardson, S.J. Schnitt, Y. Nikolsky, R.S. Gelman, K. Polyak, Molecular definition of breast tumor heterogeneity. Cancer cell 11 (2007) 259-273.

[100]A.D. Yang, F. Fan, E.R. Camp, G. van Buren, W. Liu, R. Somcio, M.J. Gray, H. Cheng, P.M. Hoff, L.M. Ellis, Chronic oxaliplatin resistance induces epithelial-tomesenchymal transition in colorectal cancer cell lines. Clin Cancer Res 12 (2006) 4147-4153.

[101]H. Kajiyama, K. Shibata, M. Terauchi, M. Yamashita, K. Ino, A. Nawa, F. Kikkawa, Chemoresistance to paclitaxel induces epithelial-mesenchymal transition and enhances metastatic potential for epithelial ovarian carcinoma cells. International journal of oncology 31 (2007) 277-283.

[102]G.Z. Cheng, J. Chan, Q. Wang, W. Zhang, C.D. Sun, L.H. Wang, Twist transcriptionally up-regulates AKT2 in breast cancer cells leading to increased migration, invasion, and resistance to paclitaxel. Cancer research 67 (2007) 1979-1987.

[103]Q.Q. Li, J.D. Xu, W.J. Wang, X.X. Cao, Q. Chen, F. Tang, Z.Q. Chen, X.P. Liu, Z.D. $\mathrm{Xu}$, Twist1-mediated adriamycin-induced epithelial-mesenchymal transition relates to multidrug resistance and invasive potential in breast cancer cells. Clin Cancer Res 15 (2009) 2657-2665.

[104]W.L. Zhuo, Y. Wang, X.L. Zhuo, Y.S. Zhang, Z.T. Chen, Short interfering RNA directed against TWIST, a novel zinc finger transcription factor, increases A549 cell sensitivity to cisplatin via MAPK/mitochondrial pathway. Biochem Biophys Res Commun 369 (2008) 1098-1102.

[105]B.G. Hollier, K. Evans, S.A. Mani, The epithelial-to-mesenchymal transition and cancer stem cells: a coalition against cancer therapies. Journal of mammary gland biology and neoplasia 14 (2009) 29-43.

[106]C. Ginestier, M.H. Hur, E. Charafe-Jauffret, F. Monville, J. Dutcher, M. Brown, J. Jacquemier, P. Viens, C.G. Kleer, S. Liu, A. Schott, D. Hayes, D. Birnbaum, M.S. Wicha, G. Dontu, ALDH1 is a marker of normal and malignant human mammary stem cells and a predictor of poor clinical outcome. Cell stem cell 1 (2007) 555-567. 
[107]M. Kakarala, M.S. Wicha, Implications of the cancer stem-cell hypothesis for breast cancer prevention and therapy. J Clin Oncol 26 (2008) 2813-2820.

[108]E.H. Huang, M.J. Hynes, T. Zhang, C. Ginestier, G. Dontu, H. Appelman, J.Z. Fields, M.S. Wicha, B.M. Boman, Aldehyde dehydrogenase 1 is a marker for normal and malignant human colonic stem cells (SC) and tracks SC overpopulation during colon tumorigenesis. Cancer research 69 (2009) 3382-3389.

[109]B. Elenbaas, L. Spirio, F. Koerner, M.D. Fleming, D.B. Zimonjic, J.L. Donaher, N.C. Popescu, W.C. Hahn, R.A. Weinberg, Human breast cancer cells generated by oncogenic transformation of primary mammary epithelial cells. Genes \& development 15 (2001) 50-65.

[110]C. Scheel, E.N. Eaton, S.H. Li, C.L. Chaffer, F. Reinhardt, K.J. Kah, G. Bell, W. Guo, J. Rubin, A.L. Richardson, R.A. Weinberg, Paracrine and autocrine signals induce and maintain mesenchymal and stem cell states in the breast. Cell 145 (2011) 926-940.

[111]S.A. Mani, W. Guo, M.J. Liao, E.N. Eaton, A. Ayyanan, A.Y. Zhou, M. Brooks, F. Reinhard, C.C. Zhang, M. Shipitsin, L.L. Campbell, K. Polyak, C. Brisken, J. Yang, R.A. Weinberg, The epithelial-mesenchymal transition generates cells with properties of stem cells. Cell 133 (2008) 704-715.

[112]B. Elenbaas, R.A. Weinberg, Heterotypic signaling between epithelial tumor cells and fibroblasts in carcinoma formation. Exp Cell Res 264 (2001) 169-184.

[113]Millipore, Guava ${ }^{\circledR}$ Multi Caspase FAM Kit. (2005).

[114]C.A. Schneider, W.S. Rasband, K.W. Eliceiri, NIH Image to ImageJ: 25 years of image analysis. Nat Methods 9 (2012) 671-675.

[115]A.C. Ruifrok, D.A. Johnston, Quantification of histochemical staining by color deconvolution. Anal Quant Cytol Histol 23 (2001) 291-299.

[116]T. Ishiguro, J. Saitoh, H. Yawata, H. Yamagishi, S. Iwasaki, Y. Mitoma, Homogeneous quantitative assay of hepatitis $\mathrm{C}$ virus RNA by polymerase chain reaction in the presence of a fluorescent intercalater. Anal Biochem 229 (1995) 207-213.

[117]K.J. Livak, T.D. Schmittgen, Analysis of relative gene expression data using real-time quantitative PCR and the 2(-Delta Delta C(T)) Method. Methods 25 (2001) 402-408.

[118]P. Nehls, J. Adamkiewicz, M.F. Rajewsky, Immuno-slot-blot: a highly sensitive immunoassay for the quantitation of carcinogen-modified nucleosides in DNA. $J$ Cancer Res Clin Oncol 108 (1984) 23-29.

[119]A.L. Shapiro, E. Vinuela, J.V. Maizel, Jr., Molecular weight estimation of polypeptide chains by electrophoresis in SDS-polyacrylamide gels. Biochem Biophys Res Commun 28 (1967) 815-820.

[120]U.K. Laemmli, Cleavage of structural proteins during the assembly of the head of bacteriophage T4. Nature 227 (1970) 680-685.

[121]M. Bittner, P. Kupferer, C.F. Morris, Electrophoretic transfer of proteins and nucleic acids from slab gels to diazobenzyloxymethyl cellulose or nitrocellulose sheets. Anal Biochem 102 (1980) 459-471.

[122]T. Brabletz, A. Jung, S. Reu, M. Porzner, F. Hlubek, L.A. Kunz-Schughart, R. Knuechel, T. Kirchner, Variable beta-catenin expression in colorectal cancers indicates tumor progression driven by the tumor environment. Proceedings of the National Academy of Sciences of the United States of America 98 (2001) 1035610361.

[123]S. Vega, A.V. Morales, O.H. Ocana, F. Valdes, I. Fabregat, M.A. Nieto, Snail blocks the cell cycle and confers resistance to cell death. Genes \& development 18 (2004) 11311143.

[124]P. Borst, S. Rottenberg, J. Jonkers, How do real tumors become resistant to cisplatin? Cell Cycle 7 (2008) 1353-1359. 
[125]V. Gagnon, I. Mathieu, E. Sexton, K. Leblanc, E. Asselin, AKT involvement in cisplatin chemoresistance of human uterine cancer cells. Gynecologic oncology 94 (2004) 785 795.

[126]R.K. Bikkavilli, M.E. Feigin, C.C. Malbon, p38 mitogen-activated protein kinase regulates canonical Wnt-beta-catenin signaling by inactivation of GSK3 beta. J Cell Sci 121 (2008) 3598-3607.

[127]S. Schutze, K. Wiegmann, T. Machleidt, M. Kronke, TNF-induced activation of NFkappa B. Immunobiology 193 (1995) 193-203.

[128]L.H. Boise, M. Gonzalez-Garcia, C.E. Postema, L. Ding, T. Lindsten, L.A. Turka, X. Mao, G. Nunez, C.B. Thompson, bcl-x, a bcl-2-related gene that functions as a dominant regulator of apoptotic cell death. Cell 74 (1993) 597-608.

[129]T.G. Obrig, W.J. Culp, W.L. McKeehan, B. Hardesty, The mechanism by which cycloheximide and related glutarimide antibiotics inhibit peptide synthesis on reticulocyte ribosomes. The Journal of biological chemistry 246 (1971) 174-181.

[130]M.E. Peter, Let-7 and miR-200 microRNAs: guardians against pluripotency and cancer progression. Cell Cycle 8 (2009) 843-852.

[131]C.P. Bracken, P.A. Gregory, N. Kolesnikoff, A.G. Bert, J. Wang, M.F. Shannon, G.J. Goodall, A double-negative feedback loop between ZEB1-SIP1 and the microRNA200 family regulates epithelial-mesenchymal transition. Cancer research 68 (2008) 7846-7854.

[132]F.J. Rauscher, D.R. Cohen, T. Curran, T.J. Bos, P.K. Vogt, D. Bohmann, R. Tjian, B.R. Franza, Fos-Associated Protein-P39 Is the Product of the Jun Proto-Oncogene. Science 240 (1988) 1010-1016.

[133]L. Sevilla, A. Zaldumbide, P. Pognonec, K.E. Boulukos, Transcriptional regulation of the bcl-x gene encoding the anti-apoptotic Bcl-x(L) protein by Ets, Rel/NF kappa B, STAT and AP1 transcription factor families. Histol Histopathol 16 (2001) 595-601.

[134]E. Shaulian, M. Karin, AP-1 as a regulator of cell life and death. Nature cell biology 4 (2002) E131-E136.

[135]P. Gao, C. Bauvy, S. Souquere, G. Tonelli, L. Liu, Y. Zhu, Z. Qiao, D. Bakula, T. Proikas-Cezanne, G. Pierron, P. Codogno, Q. Chen, M. Mehrpour, The Bcl-2 homology domain 3 mimetic gossypol induces both Beclin 1-dependent and Beclin 1independent cytoprotective autophagy in cancer cells. The Journal of biological chemistry 285 (2010) 25570-25581.

[136]J. Lian, X. Wu, F. He, D. Karnak, W. Tang, Y. Meng, D. Xiang, M. Ji, T.S. Lawrence, $\mathrm{L}$. $\mathrm{Xu}, \mathrm{A}$ natural $\mathrm{BH} 3$ mimetic induces autophagy in apoptosis-resistant prostate cancer via modulating Bcl-2-Beclin1 interaction at endoplasmic reticulum. Cell death and differentiation 18 (2011) 60-71.

[137]E. Caldas-Lopes, L. Cerchietti, J.H. Ahn, C.C. Clement, A.I. Robles, A. Rodina, K. Moulick, T. Taldone, A. Gozman, Y. Guo, N. Wu, E. de Stanchina, J. White, S.S. Gross, Y. Ma, L. Varticovski, A. Melnick, G. Chiosis, Hsp90 inhibitor PU-H71, a multimodal inhibitor of malignancy, induces complete responses in triple-negative breast cancer models. Proceedings of the National Academy of Sciences of the United States of America 106 (2009) 8368-8373.

[138]A. Kamal, F.J. Burrows, Hsp90 inhibitors as selective anticancer drugs. Discovery medicine 4 (2004) 277-280.

[139]V.P. Tryndyak, F.A. Beland, I.P. Pogribny, E-cadherin transcriptional down-regulation by epigenetic and microRNA-200 family alterations is related to mesenchymal and drug-resistant phenotypes in human breast cancer cells. International journal of cancer. Journal international du cancer 126 (2010) 2575-2583.

[140]M.K. Asiedu, J.N. Ingle, M.D. Behrens, D.C. Radisky, K.L. Knutson, TGFbeta/TNF(alpha)-mediated epithelial-mesenchymal transition generates breast 
cancer stem cells with a claudin-low phenotype. Cancer research 71 (2011) 47074719.

[141]T. Arumugam, V. Ramachandran, K.F. Fournier, H. Wang, L. Marquis, J.L. Abbruzzese, G.E. Gallick, C.D. Logsdon, D.J. McConkey, W. Choi, Epithelial to mesenchymal transition contributes to drug resistance in pancreatic cancer. Cancer research 69 (2009) 5820-5828.

[142]A.E. Sayan, T.R. Griffiths, R. Pal, G.J. Browne, A. Ruddick, T. Yagci, R. Edwards, N.J. Mayer, H. Qazi, S. Goyal, S. Fernandez, K. Straatman, G.D. Jones, K.J. Bowman, A. Colquhoun, J.K. Mellon, M. Kriajevska, E. Tulchinsky, SIP1 protein protects cells from DNA damage-induced apoptosis and has independent prognostic value in bladder cancer. Proceedings of the National Academy of Sciences of the United States of America 106 (2009) 14884-14889.

[143]J. Filmus, A.I. Robles, W. Shi, M.J. Wong, L.L. Colombo, C.J. Conti, Induction of cyclin D1 overexpression by activated ras. Oncogene 9 (1994) 3627-3633.

[144]K.E. Miletti-Gonzalez, S. Chen, N. Muthukumaran, G.N. Saglimbeni, X. Wu, J. Yang, K. Apolito, W.J. Shih, W.N. Hait, L. Rodriguez-Rodriguez, The CD44 receptor interacts with P-glycoprotein to promote cell migration and invasion in cancer. Cancer research 65 (2005) 6660-6667.

[145]M. Zoller, CD44: can a cancer-initiating cell profit from an abundantly expressed molecule? Nat Rev Cancer 11 (2011) 254-267.

[146]S.D. Vangipuram, S.A. Buck, W.D. Lyman, Wnt pathway activity confers chemoresistance to cancer stem-like cells in a neuroblastoma cell line. Tumour biology : the journal of the International Society for Oncodevelopmental Biology and Medicine 33 (2012) 2173-2183.

[147]N. Chikazawa, H. Tanaka, T. Tasaka, M. Nakamura, M. Tanaka, H. Onishi, M. Katano, Inhibition of Wnt signaling pathway decreases chemotherapy-resistant side-population colon cancer cells. Anticancer research 30 (2010) 2041-2048.

[148]N. Dumont, M.B. Wilson, Y.G. Crawford, P.A. Reynolds, M. Sigaroudinia, T.D. Tlsty, Sustained induction of epithelial to mesenchymal transition activates DNA methylation of genes silenced in basal-like breast cancers. Proceedings of the National Academy of Sciences of the United States of America 105 (2008) 14867-14872.

[149]L. Xie, B.K. Law, A.M. Chytil, K.A. Brown, M.E. Aakre, H.L. Moses, Activation of the Erk pathway is required for TGF-beta1-induced EMT in vitro. Neoplasia 6 (2004) 603-610.

[150]J.W. Antoon, R. Lai, A.P. Struckhoff, A.M. Nitschke, S. Elliott, E.C. Martin, L.V. Rhodes, N.S. Yoon, V.A. Salvo, B. Shan, B.S. Beckman, K.P. Nephew, M.E. Burow, Altered death receptor signaling promotes epithelial-to-mesenchymal transition and acquired chemoresistance. Scientific reports 2 (2012) 539.

[151]H. Bai, K. Chen, Y.X. Gao, M. Arzigian, Y.L. Xie, C. Malcosky, Y.G. Yang, W.S. Wu, Z.Z. Wang, Bcl-xL enhances single-cell survival and expansion of human embryonic stem cells without affecting self-renewal. Stem cell research 8 (2012) 26-37.

[152]S. Krajewski, M. Krajewska, B.C. Turner, C. Pratt, B. Howard, J.M. Zapata, V. Frenkel, S. Robertson, Y. Ionov, H. Yamamoto, M. Perucho, S. Takayama, J.C. Reed, Prognostic significance of apoptosis regulators in breast cancer. Endocrine-related cancer 6 (1999) 29-40.

[153]D. Kunze, K. Erdmann, M. Froehner, M.P. Wirth, S. Fuessel, siRNA-mediated inhibition of antiapoptotic genes enhances chemotherapy efficacy in bladder cancer cells. Anticancer research 32 (2012) 4313-4318.

[154]G. Packham, E.L. White, C.M. Eischen, H. Yang, E. Parganas, J.N. Ihle, D.A. Grillot, G.P. Zambetti, G. Nunez, J.L. Cleveland, Selective regulation of Bcl-XL by a Jak 
kinase-dependent pathway is bypassed in murine hematopoietic malignancies. Genes \& development 12 (1998) 2475-2487.

[155]S.S. Martin, A.G. Ridgeway, J. Pinkas, Y. Lu, M.J. Reginato, E.Y. Koh, M. Michelman, G.Q. Daley, J.S. Brugge, P. Leder, A cytoskeleton-based functional genetic screen identifies Bcl-xL as an enhancer of metastasis, but not primary tumor growth. Oncogene 23 (2004) 4641-4645.

[156]N. Rubio, L. Espana, Y. Fernandez, J. Blanco, A. Sierra, Metastatic behavior of human breast carcinomas overexpressing the $\mathrm{Bcl}-\mathrm{x}(\mathrm{L})$ gene: a role in dormancy and organospecificity. Laboratory investigation; a journal of technical methods and pathology 81 (2001) 725-734.

[157]O.I. Olopade, M.O. Adeyanju, A.R. Safa, F. Hagos, R. Mick, C.B. Thompson, W.M. Recant, Overexpression of BCL-x protein in primary breast cancer is associated with high tumor grade and nodal metastases. The cancer journal from Scientific American 3 (1997) 230-237.

[158]Z. Madjd, A.Z. Mehrjerdi, A.M. Sharifi, S. Molanaei, S.Z. Shahzadi, M. Asadi-Lari, CD44+ cancer cells express higher levels of the anti-apoptotic protein Bcl-2 in breast tumours. Cancer immunity 9 (2009) 4.

[159]D.T. Madden, D. Davila-Kruger, S. Melov, D.E. Bredesen, Human embryonic stem cells express elevated levels of multiple pro-apoptotic BCL-2 family members. PloS one 6 (2011) e28530.

[160]Y. Li, T.G. VandenBoom, 2nd, D. Kong, Z. Wang, S. Ali, P.A. Philip, F.H. Sarkar, Upregulation of miR-200 and let-7 by natural agents leads to the reversal of epithelial-tomesenchymal transition in gemcitabine-resistant pancreatic cancer cells. Cancer research 69 (2009) 6704-6712.

[161]A. Salameh, F. Galvagni, F. Anselmi, C. De Clemente, M. Orlandini, S. Oliviero, Growth factor stimulation induces cell survival by c-Jun. ATF2-dependent activation of Bcl-XL. The Journal of biological chemistry 285 (2010) 23096-23104.

[162]R.Z. Orlowski, A.S. Baldwin, Jr., NF-kappaB as a therapeutic target in cancer. Trends in molecular medicine 8 (2002) 385-389.

[163]C.Y. Wang, M.W. Mayo, A.S. Baldwin, Jr., TNF- and cancer therapy-induced apoptosis: potentiation by inhibition of NF-kappaB. Science 274 (1996) 784-787.

[164]M.A. Huber, N. Azoitei, B. Baumann, S. Grunert, A. Sommer, H. Pehamberger, N. Kraut, H. Beug, T. Wirth, NF-kappaB is essential for epithelial-mesenchymal transition and metastasis in a model of breast cancer progression. The Journal of clinical investigation 114 (2004) 569-581.

[165]D. Durie, M. Hatzoglou, P. Chakraborty, M. Holcik, HuR controls mitochondrial morphology through the regulation of Bcl-xL translation. Translation 1 (2013) e23980.

[166]A. Yoon, G. Peng, Y. Brandenburger, O. Zollo, W. Xu, E. Rego, D. Ruggero, Impaired control of IRES-mediated translation in X-linked dyskeratosis congenita. Science 312 (2006) 902-906.

[167]Z. Huang, Bcl-2 family proteins as targets for anticancer drug design. Oncogene 19 (2000) 6627-6631.

[168]S. Wang, D. Yang, M.E. Lippman, Targeting Bcl-2 and Bcl-XL with nonpeptidic smallmolecule antagonists. Semin Oncol 30 (2003) 133-142.

[169]K.G. Wolter, S.J. Wang, B.S. Henson, S. Wang, K.A. Griffith, B. Kumar, J. Chen, T.E. Carey, C.R. Bradford, N.J. D'Silva, (-)-gossypol inhibits growth and promotes apoptosis of human head and neck squamous cell carcinoma in vivo. Neoplasia 8 (2006) 163-172.

[170]M.C. Maiuri, G. Le Toumelin, A. Criollo, J.C. Rain, F. Gautier, P. Juin, E. Tasdemir, G. Pierron, K. Troulinaki, N. Tavernarakis, J.A. Hickman, O. Geneste, G. Kroemer, 
Functional and physical interaction between $\mathrm{Bcl}-\mathrm{X}(\mathrm{L})$ and a BH3-like domain in Beclin-1. The EMBO journal 26 (2007) 2527-2539.

[171]R. Nimmanapalli, E. O'Bryan, D. Kuhn, H. Yamaguchi, H.G. Wang, K.N. Bhalla, Regulation of 17-AAG-induced apoptosis: role of Bcl-2, Bcl-XL, and Bax downstream of 17-AAG-mediated down-regulation of Akt, Raf-1, and Src kinases. Blood 102 (2003) 269-275.

[172]R. Kumaraguruparan, D. Karunagaran, C. Balachandran, B.M. Manohar, S. Nagini, Of humans and canines: a comparative evaluation of heat shock and apoptosis-associated proteins in mammary tumors. Clinica chimica acta; international journal of clinical chemistry 365 (2006) 168-176.

[173]A.S. Sreedhar, P. Csermely, Heat shock proteins in the regulation of apoptosis: new strategies in tumor therapy: a comprehensive review. Pharmacology \& therapeutics 101 (2004) 227-257.

[174]J. Beliakoff, L. Whitesell, Hsp90: an emerging target for breast cancer therapy. Anticancer drugs 15 (2004) 651-662.

[175]H.M. Beere, B.B. Wolf, K. Cain, D.D. Mosser, A. Mahboubi, T. Kuwana, P. Tailor, R.I. Morimoto, G.M. Cohen, D.R. Green, Heat-shock protein 70 inhibits apoptosis by preventing recruitment of procaspase- 9 to the Apaf-1 apoptosome. Nature cell biology 2 (2000) 469-475.

[176]D. Picard, HSP90 interactors, in: D. Picard, (Ed.), http://www.picard.ch/downloads/Hsp90interactors.pdf, 2013.

[177]C. Perotti, R. Liu, C.T. Parusel, N. Bocher, J. Schultz, P. Bork, E. Pfitzner, B. Groner, C.S. Shemanko, Heat shock protein-90-alpha, a prolactin-STAT5 target gene identified in breast cancer cells, is involved in apoptosis regulation. Breast cancer research : BCR 10 (2008) R94.

[178]S.Z. Usmani, R. Bona, Z. Li, 17 AAG for HSP90 inhibition in cancer--from bench to bedside. Current molecular medicine 9 (2009) 654-664.

[179]S. Kummar, M.E. Gutierrez, E.R. Gardner, X. Chen, W.D. Figg, M. Zajac-Kaye, M. Chen, S.M. Steinberg, C.A. Muir, M.A. Yancey, Y.R. Horneffer, L. Juwara, G. Melillo, S.P. Ivy, M. Merino, L. Neckers, P.S. Steeg, B.A. Conley, G. Giaccone, J.H. Doroshow, A.J. Murgo, Phase I trial of 17-dimethylaminoethylamino-17demethoxygeldanamycin (17-DMAG), a heat shock protein inhibitor, administered twice weekly in patients with advanced malignancies. Eur J Cancer 46 (2010) 340347.

[180]E.R. Glaze, A.L. Lambert, A.C. Smith, J.G. Page, W.D. Johnson, D.L. McCormick, A.P. Brown, B.S. Levine, J.M. Covey, M.J. Egorin, J.L. Eiseman, J.L. Holleran, E.A. Sausville, J.E. Tomaszewski, Preclinical toxicity of a geldanamycin analog, 17(dimethylaminoethylamino)-17-demethoxygeldanamycin (17-DMAG), in rats and dogs: potential clinical relevance. Cancer chemotherapy and pharmacology 56 (2005) 637-647.

[181]V. Smith, E.A. Sausville, R.F. Camalier, H.H. Fiebig, A.M. Burger, Comparison of 17dimethylaminoethylamino-17-demethoxy-geldanamycin (17DMAG) and 17allylamino-17-demethoxygeldanamycin (17AAG) in vitro: effects on Hsp90 and client proteins in melanoma models. Cancer chemotherapy and pharmacology 56 (2005) 126-137.

[182]M. Hollingshead, M. Alley, A.M. Burger, S. Borgel, C. Pacula-Cox, H.H. Fiebig, E.A. Sausville, In vivo antitumor efficacy of 17-DMAG (17-dimethylaminoethylamino-17demethoxygeldanamycin hydrochloride), a water-soluble geldanamycin derivative. Cancer chemotherapy and pharmacology 56 (2005) 115-125.

[183]J.L. Eiseman, J. Lan, T.F. Lagattuta, D.R. Hamburger, E. Joseph, J.M. Covey, M.J. Egorin, Pharmacokinetics and pharmacodynamics of 17-demethoxy 17-[[(2- 
dimethylamino)ethyl]amino]geldanamycin (17DMAG, NSC 707545) in C.B-17 SCID mice bearing MDA-MB-231 human breast cancer xenografts. Cancer chemotherapy and pharmacology 55 (2005) 21-32.

[184]Dharmendra, K.R. Chatterjee, Prognostic value of the lepromin test in contacts of leprosy cases. 1955. Indian journal of leprosy 63 (1991) 293-296.

[185]P.B. Gupta, T.T. Onder, G. Jiang, K. Tao, C. Kuperwasser, R.A. Weinberg, E.S. Lander, Identification of selective inhibitors of cancer stem cells by high-throughput screening. Cell 138 (2009) 645-659.

[186]A. Huczynski, Salinomycin: a new cancer drug candidate. Chem Biol Drug Des 79 (2012) 235-238.

[187]W. Boehmerle, M. Endres, Salinomycin induces calpain and cytochrome c-mediated neuronal cell death. Cell death \& disease 2 (2011) e168.

[188]P. Story, A. Doube, A case of human poisoning by salinomycin, an agricultural antibiotic. The New Zealand medical journal 117 (2004) U799.

[189]T. Brabletz, A. Jung, S. Spaderna, F. Hlubek, T. Kirchner, Opinion: migrating cancer stem cells - an integrated concept of malignant tumour progression. Nat Rev Cancer 5 (2005) 744-749.

[190]M. Yu, A. Bardia, B.S. Wittner, S.L. Stott, M.E. Smas, D.T. Ting, S.J. Isakoff, J.C. Ciciliano, M.N. Wells, A.M. Shah, K.F. Concannon, M.C. Donaldson, L.V. Sequist, E. Brachtel, D. Sgroi, J. Baselga, S. Ramaswamy, M. Toner, D.A. Haber, S. Maheswaran, Circulating breast tumor cells exhibit dynamic changes in epithelial and mesenchymal composition. Science 339 (2013) 580-584.

[191]I. International Stem Cell, K. Amps, P.W. Andrews, G. Anyfantis, L. Armstrong, S. Avery, H. Baharvand, J. Baker, D. Baker, M.B. Munoz, S. Beil, N. Benvenisty, D. Ben-Yosef, J.C. Biancotti, A. Bosman, R.M. Brena, D. Brison, G. Caisander, M.V. Camarasa, J. Chen, E. Chiao, Y.M. Choi, A.B. Choo, D. Collins, A. Colman, J.M. Crook, G.Q. Daley, A. Dalton, P.A. De Sousa, C. Denning, J. Downie, P. Dvorak, K.D. Montgomery, A. Feki, A. Ford, V. Fox, A.M. Fraga, T. Frumkin, L. Ge, P.J. Gokhale, T. Golan-Lev, H. Gourabi, M. Gropp, G. Lu, A. Hampl, K. Harron, L. Healy, W. Herath, F. Holm, O. Hovatta, J. Hyllner, M.S. Inamdar, A.K. Irwanto, T. Ishii, M. Jaconi, Y. Jin, S. Kimber, S. Kiselev, B.B. Knowles, O. Kopper, V. Kukharenko, A. Kuliev, M.A. Lagarkova, P.W. Laird, M. Lako, A.L. Laslett, N. Lavon, D.R. Lee, J.E. Lee, C. Li, L.S. Lim, T.E. Ludwig, Y. Ma, E. Maltby, I. Mateizel, Y. Mayshar, M. Mileikovsky, S.L. Minger, T. Miyazaki, S.Y. Moon, H. Moore, C. Mummery, A. Nagy, N. Nakatsuji, K. Narwani, S.K. Oh, S.K. Oh, C. Olson, T. Otonkoski, F. Pan, I.H. Park, S. Pells, M.F. Pera, L.V. Pereira, O. Qi, G.S. Raj, B. Reubinoff, A. Robins, P. Robson, J. Rossant, G.H. Salekdeh, T.C. Schulz, K. Sermon, J. Sheik Mohamed, H. Shen, E. Sherrer, K. Sidhu, S. Sivarajah, H. Skottman, C. Spits, G.N. Stacey, R. Strehl, N. Strelchenko, H. Suemori, B. Sun, R. Suuronen, K. Takahashi, T. Tuuri, P. Venu, Y. Verlinsky, D. Ward-van Oostwaard, D.J. Weisenberger, Y. Wu, S. Yamanaka, L. Young, Q. Zhou, Screening ethnically diverse human embryonic stem cells identifies a chromosome 20 minimal amplicon conferring growth advantage. Nature biotechnology 29 (2011) 1132-1144.

[192]N.Y. Chia, Y.S. Chan, B. Feng, X. Lu, Y.L. Orlov, D. Moreau, P. Kumar, L. Yang, J. Jiang, M.S. Lau, M. Huss, B.S. Soh, P. Kraus, P. Li, T. Lufkin, B. Lim, N.D. Clarke, F. Bard, H.H. Ng, A genome-wide RNAi screen reveals determinants of human embryonic stem cell identity. Nature 468 (2010) 316-320.

[193]R. Beroukhim, C.H. Mermel, D. Porter, G. Wei, S. Raychaudhuri, J. Donovan, J. Barretina, J.S. Boehm, J. Dobson, M. Urashima, K.T. Mc Henry, R.M. Pinchback, A.H. Ligon, Y.J. Cho, L. Haery, H. Greulich, M. Reich, W. Winckler, M.S. Lawrence, B.A. Weir, K.E. Tanaka, D.Y. Chiang, A.J. Bass, A. Loo, C. Hoffman, J. Prensner, T. 
Liefeld, Q. Gao, D. Yecies, S. Signoretti, E. Maher, F.J. Kaye, H. Sasaki, J.E. Tepper, J.A. Fletcher, J. Tabernero, J. Baselga, M.S. Tsao, F. Demichelis, M.A. Rubin, P.A. Janne, M.J. Daly, C. Nucera, R.L. Levine, B.L. Ebert, S. Gabriel, A.K. Rustgi, C.R. Antonescu, M. Ladanyi, A. Letai, L.A. Garraway, M. Loda, D.G. Beer, L.D. True, A. Okamoto, S.L. Pomeroy, S. Singer, T.R. Golub, E.S. Lander, G. Getz, W.R. Sellers, M. Meyerson, The landscape of somatic copy-number alteration across human cancers. Nature 463 (2010) 899-905.

[194]M. Vogler, D. Dinsdale, M.J. Dyer, G.M. Cohen, Bcl-2 inhibitors: small molecules with a big impact on cancer therapy. Cell death and differentiation 16 (2009) 360-367.

[195]S. Modi, A.T. Stopeck, M.S. Gordon, D. Mendelson, D.B. Solit, R. Bagatell, W. Ma, J. Wheler, N. Rosen, L. Norton, G.F. Cropp, R.G. Johnson, A.L. Hannah, C.A. Hudis, Combination of trastuzumab and tanespimycin (17-AAG, KOS-953) is safe and active in trastuzumab-refractory HER-2 overexpressing breast cancer: a phase I doseescalation study. J Clin Oncol 25 (2007) 5410-5417.

[196]P.N. Munster, A. Basso, D. Solit, L. Norton, N. Rosen, Modulation of Hsp90 function by ansamycins sensitizes breast cancer cells to chemotherapy-induced apoptosis in an RB- and schedule-dependent manner. See: E. A. Sausville, Combining cytotoxics and 17-allylamino, 17-demethoxygeldanamycin: sequence and tumor biology matters, Clin. Cancer Res., 7: 2155-2158, 2001. Clin Cancer Res 7 (2001) 2228-2236.

[197]U. Banerji, N. Sain, S.Y. Sharp, M. Valenti, Y. Asad, R. Ruddle, F. Raynaud, M. Walton, S.A. Eccles, I. Judson, A.L. Jackman, P. Workman, An in vitro and in vivo study of the combination of the heat shock protein inhibitor 17-allylamino-17demethoxygeldanamycin and carboplatin in human ovarian cancer models. Cancer chemotherapy and pharmacology 62 (2008) 769-778.

[198]N.C.I. (NCI), R-(-)-Gossypol acetic acid, Cisplatin, and Etoposide in treating patients with advanced solid tumors or extensive stage small cell lung cancer, in, U.S. National Institutes of Health, ClinicalTrials.gov, 2007.

[199]N.C.I. (NCI), Gossypol, Paclitaxel, and Carboplatin in treating patients with solid tumors that are metastatic or cannot be removed by surgery, in, U.S. National Institutes of Health, ClinicalTrials.gov, 2009.

[200]X. Wang, J. Wang, S.C. Wong, L.S. Chow, J.M. Nicholls, Y.C. Wong, Y. Liu, D.L. Kwong, J.S. Sham, S.W. Tsa, Cytotoxic effect of gossypol on colon carcinoma cells. Life sciences 67 (2000) 2663-2671.

[201]M. Zhang, H. Liu, R. Guo, Y. Ling, X. Wu, B. Li, P.P. Roller, S. Wang, D. Yang, Molecular mechanism of gossypol-induced cell growth inhibition and cell death of HT-29 human colon carcinoma cells. Biochemical pharmacology 66 (2003) 93-103.

[202]C. Van Poznak, A.D. Seidman, M.M. Reidenberg, M.M. Moasser, N. Sklarin, K. Van Zee, P. Borgen, M. Gollub, D. Bacotti, T.J. Yao, R. Bloch, M. Ligueros, M. Sonenberg, L. Norton, C. Hudis, Oral gossypol in the treatment of patients with refractory metastatic breast cancer: a phase I/II clinical trial. Breast Cancer Res Treat 66 (2001) 239-248.

[203]L. Blackstaffe, M.D. Shelley, R.G. Fish, Cytotoxicity of gossypol enantiomers and its quinone metabolite gossypolone in melanoma cell lines. Melanoma research 7 (1997) 364-372.

[204]P. Bushunow, M.M. Reidenberg, J. Wasenko, J. Winfield, B. Lorenzo, S. Lemke, B. Himpler, R. Corona, T. Coyle, Gossypol treatment of recurrent adult malignant gliomas. Journal of neuro-oncology 43 (1999) 79-86. 


\section{Appendix}

\section{List of differentially regulated genes in MSP cells compared to $24^{+}$HMLE cells. Micro} array results.

The table includes the name of the differentially regulated genes and the NCBI number. Induction of gene expression in MSP cells relative to $24^{+}$HMLE cells is shown as log2, since the threshold was set at 2. Positive values indicate up-regulation in MSP cells and negative values the down-regulation in MSP cells compared to $24^{+}$HMLE cells.

The fold induction can be calculated by $2^{\mathrm{x}}$.

\begin{tabular}{|c|c|c|c|}
\hline Symbol & Gene & $\log F C$ & FDR \\
\hline ALDH1A3 & 220 & 1,507083 & $0,38 \%$ \\
\hline ACSS2 & 55902 & 1,100465 & $0,10 \%$ \\
\hline PFKFB2 & 5208 & $-1,78158$ & $0,01 \%$ \\
\hline ACSL1 & 2180 & 1,771212 & $0,03 \%$ \\
\hline CPT1A & 1374 & 1,359796 & $0,11 \%$ \\
\hline CYP1B1 & 1545 & 2,349792 & $0,01 \%$ \\
\hline SULT1E1 & 6783 & 1,068866 & $0,33 \%$ \\
\hline UQCRQ & 27089 & 1,224993 & $0,06 \%$ \\
\hline PDE7A & 5150 & $-1,08929$ & $0,13 \%$ \\
\hline PDE9A & 5152 & 1,535564 & $0,04 \%$ \\
\hline POLR3GL & 84265 & 1,196115 & $0,74 \%$ \\
\hline ENPP1 & 5167 & 3,197189 & $0,00 \%$ \\
\hline AK5 & 26289 & 1,033676 & $0,06 \%$ \\
\hline DPYD & 1806 & 1,395174 & $0,01 \%$ \\
\hline UPP1 & 7378 & $-1,26477$ & $0,14 \%$ \\
\hline GLUL & 2752 & 2,185174 & $0,00 \%$ \\
\hline $\mathrm{CTH}$ & 1491 & $-1,27519$ & $1,93 \%$ \\
\hline MAOA & 4128 & 1,174297 & $1,49 \%$ \\
\hline BCAT1 & 586 & $-2,21221$ & $0,00 \%$ \\
\hline AOX1 & 316 & 3,639332 & $0,00 \%$ \\
\hline LARS & 51520 & 1,000945 & $0,04 \%$ \\
\hline HNMT & 3176 & 1,637541 & $0,38 \%$ \\
\hline GCLC & 2729 & $-1,05815$ & $0,19 \%$ \\
\hline AGL & 178 & $-1,28933$ & $0,03 \%$ \\
\hline ST6GAL1 & 6480 & $-1,16834$ & $0,64 \%$ \\
\hline GALNT10 & 55568 & 1,257673 & $0,44 \%$ \\
\hline GALNT3 & 2591 & $-1,59177$ & $0,01 \%$ \\
\hline GALNT5 & 11227 & $-1,82909$ & $0,11 \%$ \\
\hline GCNT1 & 2650 & 1,444338 & $0,04 \%$ \\
\hline GCNT4 & 51301 & $-1,50139$ & $0,03 \%$ \\
\hline DSE & 29940 & $-1,27181$ & $0,61 \%$ \\
\hline CHST11 & 50515 & $-1,30296$ & $4,49 \%$ \\
\hline B4GALT4 & 8702 & $-1,13215$ & $1,17 \%$ \\
\hline HS3ST2 & 9956 & -1.21478 & $0.23 \%$ \\
\hline EXT1 & 2131 & $-1,27528$ & $0,04 \%$ \\
\hline
\end{tabular}




\begin{tabular}{|c|c|c|c|}
\hline DGKB & 1607 & 1,206388 & $0,57 \%$ \\
\hline PLCE1 & 51196 & 1,192281 & $0,27 \%$ \\
\hline INPP4B & 8821 & $-1,08805$ & $0,28 \%$ \\
\hline PLA2G4A & 5321 & $-3,95197$ & $0,00 \%$ \\
\hline ETNK2 & 55224 & 1,077889 & $2,67 \%$ \\
\hline PCYT1B & 9468 & $-1,05496$ & $0,18 \%$ \\
\hline CDS1 & 1040 & $-2,31769$ & $0,00 \%$ \\
\hline ENPP2 & 5168 & 5,683607 & $0,00 \%$ \\
\hline PLA2G7 & 7941 & $-2,02106$ & $0,85 \%$ \\
\hline PTGS2 & 5743 & $-2,45948$ & $0,02 \%$ \\
\hline FADS2 & 9415 & 3,452264 & $0,00 \%$ \\
\hline ACER3 & 55331 & $-1,02918$ & $0,19 \%$ \\
\hline ST3GAL5 & 8869 & 1,290383 & $0,09 \%$ \\
\hline NNMT & 4837 & 1,597558 & $0,00 \%$ \\
\hline $\mathrm{GCH} 1$ & 2643 & $-1,68895$ & $0,01 \%$ \\
\hline LRAT & 9227 & $-2,73782$ & $0,01 \%$ \\
\hline CA12 & 771 & $-1,27221$ & $0,07 \%$ \\
\hline CA2 & 760 & $-1,82728$ & $0,16 \%$ \\
\hline CA8 & 767 & 1,1996 & $1,56 \%$ \\
\hline CES1 & 1066 & 1,556555 & $0,16 \%$ \\
\hline FADS1 & 3992 & 1,817718 & $0,02 \%$ \\
\hline PON2 & 5445 & 1,478673 & $0,09 \%$ \\
\hline ABCA1 & 19 & $-1,92064$ & $0,43 \%$ \\
\hline ABCA12 & 26154 & $-3,13788$ & $0,00 \%$ \\
\hline ABCA6 & 23460 & 3,022089 & $0,00 \%$ \\
\hline ABCG2 & 9429 & $-2,15632$ & $0,01 \%$ \\
\hline ABCD2 & 225 & 1,03692 & $0,33 \%$ \\
\hline MPHOSPH6 & 10200 & $-1,03352$ & $0,06 \%$ \\
\hline SLU7 & 10569 & 1,031951 & $0,05 \%$ \\
\hline PPARG & 5468 & 2,62103 & $0,00 \%$ \\
\hline ERCC6 & 2074 & 1,306861 & $0,01 \%$ \\
\hline CDK7 & 1022 & $-1,03282$ & $0,52 \%$ \\
\hline DUSP1 & 1843 & 2,738331 & $0,08 \%$ \\
\hline DUSP10 & 11221 & $-2,59926$ & $0,00 \%$ \\
\hline PRKCA & 5578 & 2,497344 & $0,00 \%$ \\
\hline IL1B & 3553 & $-1,70638$ & $0,04 \%$ \\
\hline IL1R1 & 3554 & 1,983528 & $0,00 \%$ \\
\hline IL1A & 3552 & $-4,93904$ & $0,00 \%$ \\
\hline RPS6KA2 & 6196 & 2,638631 & $0,00 \%$ \\
\hline FGFR2 & 2263 & $-2,2699$ & $0,04 \%$ \\
\hline MAPK13 & 5603 & $-1,09479$ & $2,45 \%$ \\
\hline FGFR1 & 2260 & 1,792861 & $0,01 \%$ \\
\hline MECOM & 2122 & $-1,56476$ & $0,02 \%$ \\
\hline PIK3R1 & 5295 & 1,270797 & $0,01 \%$ \\
\hline PAK3 & 5063 & 1,909093 & $0,01 \%$ \\
\hline GAB1 & 2549 & $-1,22068$ & $0,03 \%$ \\
\hline HBEGF & 1839 & $-1,48951$ & $0,06 \%$ \\
\hline GNA15 & 2769 & $-1,18307$ & $0,69 \%$ \\
\hline PTGFR & 5737 & 2,371151 & $0,01 \%$ \\
\hline F2R & 2149 & 1,44525 & $0,09 \%$ \\
\hline EDNRA & 1909 & 2,010692 & $0,12 \%$ \\
\hline CAMK4 & 814 & 2,606334 & $0,01 \%$ \\
\hline GNAL & 2774 & $-1,703$ & $0,67 \%$ \\
\hline EDA2R & 60401 & 1,364127 & $2,51 \%$ \\
\hline TNFRSF21 & 27242 & $-1,67118$ & $0,03 \%$ \\
\hline
\end{tabular}




\begin{tabular}{|c|c|c|c|}
\hline TNFSF15 & 9966 & $-1,2919$ & $2,31 \%$ \\
\hline CXCL16 & 58191 & $-1,7816$ & $0,35 \%$ \\
\hline LEPR & 3953 & 1,813564 & $0,03 \%$ \\
\hline KDR & 3791 & 1,638889 & $0,01 \%$ \\
\hline VEGFA & 7422 & $-1,48917$ & $0,02 \%$ \\
\hline TNFSF10 & 8743 & $-1,85949$ & $0,09 \%$ \\
\hline INHBA & 3624 & $-2,39234$ & $0,07 \%$ \\
\hline PDGFC & 56034 & 1,562102 & $0,00 \%$ \\
\hline IL18 & 3606 & $-2,4409$ & $0,01 \%$ \\
\hline IFNE & 338376 & 1,139559 & $1,54 \%$ \\
\hline IFNK & 56832 & $-2,90672$ & $0,02 \%$ \\
\hline IL20RB & 53833 & $-1,46587$ & $0,28 \%$ \\
\hline LIFR & 3977 & 1,750814 & $0,01 \%$ \\
\hline IFNGR1 & 3459 & $-1,0189$ & $0,14 \%$ \\
\hline GNG11 & 2791 & 2,061082 & $0,01 \%$ \\
\hline GNG2 & 54331 & 1,042098 & $0,24 \%$ \\
\hline ADRBK2 & 157 & $-2,13647$ & $0,00 \%$ \\
\hline LYN & 4067 & $-1,88945$ & $0,05 \%$ \\
\hline CNR1 & 1268 & 1,168863 & $0,05 \%$ \\
\hline F2RL1 & 2150 & $-1,68529$ & $0,02 \%$ \\
\hline F2RL2 & 2151 & 1,199652 & $0,29 \%$ \\
\hline HTR1B & 3351 & 2,120665 & $0,03 \%$ \\
\hline LPAR3 & 23566 & $-2,92509$ & $0,10 \%$ \\
\hline S1PR3 & 1903 & 1,926169 & $0,01 \%$ \\
\hline CALCRL & 10203 & 1,1769 & $2,37 \%$ \\
\hline LPAR1 & 1902 & 1,219848 & $0,07 \%$ \\
\hline PTTG1 & 9232 & 1,211616 & $0,09 \%$ \\
\hline SKP1 & 6500 & 1,127976 & $0,22 \%$ \\
\hline CDC25C & 995 & 1,391117 & $0,01 \%$ \\
\hline CCND2 & 894 & $-3,45301$ & $0,04 \%$ \\
\hline SFN & 2810 & $-1,16437$ & $0,15 \%$ \\
\hline E2F5 & 1875 & $-2,00685$ & $0,10 \%$ \\
\hline CCNA1 & 8900 & $-1,26002$ & $0,07 \%$ \\
\hline CCNG2 & 901 & $-1,04092$ & $2,23 \%$ \\
\hline IGFBP3 & 3486 & 1,59098 & $0,09 \%$ \\
\hline PERP & 64065 & $-2,35338$ & $0,02 \%$ \\
\hline SESN3 & 143686 & $-2,05296$ & $0,04 \%$ \\
\hline SERPINE1 & 5054 & 3,307557 & $0,00 \%$ \\
\hline KLHL13 & 90293 & $-1,49229$ & $0,05 \%$ \\
\hline NEDD4 & 4734 & 1,264956 & $0,02 \%$ \\
\hline NEDD4L & 23327 & $-1,52895$ & $0,02 \%$ \\
\hline STX3 & 6809 & $-1,20923$ & $0,35 \%$ \\
\hline AP1M2 & 10053 & $-1,48737$ & $1,09 \%$ \\
\hline CD63 & 967 & 1,207195 & $2,10 \%$ \\
\hline CTSC & 1075 & $-1,87703$ & $0,05 \%$ \\
\hline CTSL2 & 1515 & $-3,16895$ & $0,11 \%$ \\
\hline ARAP2 & 116984 & $-2,45387$ & $0,01 \%$ \\
\hline CHMP2B & 25978 & $-1,4471$ & $0,01 \%$ \\
\hline CHMP4C & 92421 & $-3,12252$ & $0,00 \%$ \\
\hline DNAJC6 & 9829 & 1,007965 & $0,13 \%$ \\
\hline PARD6G & 84552 & $-1,11573$ & $0,06 \%$ \\
\hline DNM3 & 26052 & $-1,45974$ & $0,13 \%$ \\
\hline CAB39 & 51719 & $-1,46568$ & $0,00 \%$ \\
\hline BCL2L1 & 598 & 1,111819 & $0,20 \%$ \\
\hline IRAK3 & 11213 & 1,064325 & $0,38 \%$ \\
\hline
\end{tabular}




\begin{tabular}{|c|c|c|c|}
\hline ACTA2 & 59 & 1,273341 & $0,54 \%$ \\
\hline KCNMA1 & 3778 & 4,025247 & $0,00 \%$ \\
\hline PRKCH & 5583 & $-3,71194$ & $0,00 \%$ \\
\hline DAAM1 & 23002 & $-1,33325$ & $0,04 \%$ \\
\hline LEF1 & 51176 & 2,277916 & $0,01 \%$ \\
\hline TCF7L1 & 83439 & $-1,13187$ & $1,85 \%$ \\
\hline FZD3 & 7976 & $-1,77138$ & $0,00 \%$ \\
\hline FZD6 & 8323 & $-1,23581$ & $0,06 \%$ \\
\hline ETV6 & 2120 & $-1,03001$ & $0,67 \%$ \\
\hline KAT2B & 8850 & 1,317162 & $0,01 \%$ \\
\hline HHIP & 64399 & 5,399516 & $0,00 \%$ \\
\hline DCN & 1634 & 5,949161 & $0,00 \%$ \\
\hline FST & 10468 & $-5,9779$ & $0,00 \%$ \\
\hline LTBP1 & 4052 & 2,838986 & $0,00 \%$ \\
\hline SMAD1 & 4086 & $-1,12476$ & $0,08 \%$ \\
\hline ABLIM1 & 3983 & $-2,78316$ & $0,00 \%$ \\
\hline ABLIM3 & 22885 & 3,065491 & $0,00 \%$ \\
\hline DPYSL2 & 1808 & 1,044855 & $0,73 \%$ \\
\hline EFNA5 & 1946 & 1,562579 & $0,00 \%$ \\
\hline NRP1 & 8829 & 1,309366 & $0,04 \%$ \\
\hline NTN4 & 59277 & 2,277654 & $0,02 \%$ \\
\hline SEMA3A & 10371 & $-3,22016$ & $0,00 \%$ \\
\hline SEMA3E & 9723 & $-1,6858$ & $0,07 \%$ \\
\hline SEMA5A & 9037 & 2,006641 & $0,02 \%$ \\
\hline COL5A2 & 1290 & 1,668055 & $0,01 \%$ \\
\hline TNC & 3371 & $-1,21182$ & $0,15 \%$ \\
\hline ITGA4 & 3676 & $-3,83512$ & $0,00 \%$ \\
\hline ITGB8 & 3696 & $-2,474$ & $0,00 \%$ \\
\hline ITGA2 & 3673 & $-2,45103$ & $0,03 \%$ \\
\hline ITGB6 & 3694 & $-5,24954$ & $0,00 \%$ \\
\hline LAMA3 & 3909 & $-2,74856$ & $0,02 \%$ \\
\hline LAMB3 & 3914 & $-1,49319$ & $0,47 \%$ \\
\hline LAMC2 & 3918 & $-4,05752$ & $0,01 \%$ \\
\hline SDC2 & 6383 & 1,11137 & $0,05 \%$ \\
\hline SDC3 & 9672 & 1,491069 & $0,01 \%$ \\
\hline ALCAM & 214 & 1,264535 & $0,01 \%$ \\
\hline CADM1 & 23705 & $-1,07642$ & $0,04 \%$ \\
\hline CADM3 & 57863 & 2,511207 & $0,03 \%$ \\
\hline CDH3 & 1001 & $-3,42883$ & $0,04 \%$ \\
\hline $\mathrm{CDH} 4$ & 1002 & 1,037551 & $0,88 \%$ \\
\hline CNTN1 & 1272 & $-4,84675$ & $0,00 \%$ \\
\hline NEGR1 & 257194 & 4,379052 & $0,00 \%$ \\
\hline NRCAM & 4897 & $-2,13581$ & $0,00 \%$ \\
\hline PTPRM & 5797 & 1,18753 & $0,08 \%$ \\
\hline PVRL3 & 25945 & 2,094607 & $0,00 \%$ \\
\hline CDH1 & 999 & $-5,52742$ & $0,00 \%$ \\
\hline F11R & 50848 & $-2,37166$ & $0,01 \%$ \\
\hline JAM3 & 83700 & 1,88106 & $0,00 \%$ \\
\hline CLDN1 & 9076 & $-4,65226$ & $0,01 \%$ \\
\hline OCLN & 4950 & $-3,84561$ & $0,00 \%$ \\
\hline PECAM1 & 5175 & 1,620255 & $0,08 \%$ \\
\hline $\mathrm{CDH} 2$ & 1000 & 3,538188 & $0,00 \%$ \\
\hline EPB41 & 2035 & $-2,11372$ & $0,01 \%$ \\
\hline INADL & 10207 & $-2,77523$ & $0,00 \%$ \\
\hline GJA1 & 2697 & $-2,72669$ & $0,00 \%$ \\
\hline
\end{tabular}




\begin{tabular}{|c|c|c|c|}
\hline PROS1 & 5627 & 1,591022 & $0,18 \%$ \\
\hline TFPI & 7035 & 4,885055 & $0,00 \%$ \\
\hline CD59 & 966 & 1,363465 & $0,09 \%$ \\
\hline C5 & 727 & 1,075757 & $0,13 \%$ \\
\hline C3 & 718 & $-2,68391$ & $0,04 \%$ \\
\hline IFI30 & 10437 & $-1,1818$ & $0,10 \%$ \\
\hline MME & 4311 & 3,05898 & $0,00 \%$ \\
\hline TICAM2 & 353376 & 1,377929 & $0,16 \%$ \\
\hline TLR2 & 7097 & 1,011527 & $0,27 \%$ \\
\hline TLR4 & 7099 & 2,457467 & $0,01 \%$ \\
\hline TAB3 & 257397 & $-1,0933$ & $0,05 \%$ \\
\hline TNFAIP3 & 7128 & $-1,17358$ & $1,77 \%$ \\
\hline IFIH1 & 64135 & $-3,06364$ & $0,00 \%$ \\
\hline RNF125 & 54941 & $-1,74584$ & $0,01 \%$ \\
\hline DDX58 & 23586 & $-1,63902$ & $0,00 \%$ \\
\hline SPRY2 & 10253 & 1,231053 & $0,50 \%$ \\
\hline CD9 & 928 & $-1,1103$ & $0,15 \%$ \\
\hline SYK & 6850 & $-1,4786$ & $0,23 \%$ \\
\hline DAPP1 & 27071 & $-4,25505$ & $0,00 \%$ \\
\hline THY1 & 7070 & 1,578185 & $0,17 \%$ \\
\hline MAGED1 & 9500 & 1,050891 & $0,11 \%$ \\
\hline CLCA2 & 9635 & $-4,1538$ & $0,00 \%$ \\
\hline OR5P3 & 120066 & $-1,05539$ & $0,25 \%$ \\
\hline TAS2R10 & 50839 & 1,329106 & $0,69 \%$ \\
\hline CYFIP2 & 26999 & 1,421903 & $0,09 \%$ \\
\hline ARHGEF6 & 9459 & 1,512228 & $0,91 \%$ \\
\hline FLOT1 & 10211 & 1,017955 & $0,44 \%$ \\
\hline PPP1R3C & 5507 & 2,585413 & $0,12 \%$ \\
\hline NR5A2 & 2494 & 1,433663 & $0,03 \%$ \\
\hline BACE2 & 25825 & $-1,0344$ & $2,17 \%$ \\
\hline SNCA & 6622 & $-2,02305$ & $0,01 \%$ \\
\hline DERL1 & 79139 & $-1,32117$ & $0,01 \%$ \\
\hline NEFM & 4741 & $-4,20615$ & $0,00 \%$ \\
\hline DCTN4 & 51164 & 1,099948 & $0,09 \%$ \\
\hline KRT18 & 3875 & $-1,42752$ & $3,54 \%$ \\
\hline DAPK1 & 1612 & 1,747935 & $0,13 \%$ \\
\hline JUP & 3728 & $-1,56159$ & $0,07 \%$ \\
\hline DSC2 & 1824 & $-6,49542$ & $0,00 \%$ \\
\hline DSG2 & 1829 & $-1,29254$ & $0,01 \%$ \\
\hline DSP & 1832 & $-6,88033$ & $0,00 \%$ \\
\hline A2ML1 & 144568 & $-2,96629$ & $0,07 \%$ \\
\hline ABI3BP & 25890 & 3,253779 & $0,06 \%$ \\
\hline ADAM32 & 203102 & 1,573119 & $0,03 \%$ \\
\hline ADAMTS1 & 9510 & 1,431545 & $0,09 \%$ \\
\hline ADAMTSL1 & 92949 & 1,281668 & $0,02 \%$ \\
\hline AFAP1L2 & 84632 & $-1,76564$ & $0,00 \%$ \\
\hline AGPAT9 & 84803 & $-2,13294$ & $0,21 \%$ \\
\hline ANGPT1 & 284 & 2,665148 & $0,09 \%$ \\
\hline ANK2 & 287 & $-1,03779$ & $0,36 \%$ \\
\hline ANKRD1 & 27063 & 3,940318 & $0,00 \%$ \\
\hline ANKRD13A & 88455 & $-1,16515$ & $0,16 \%$ \\
\hline ANKRD18A & 253650 & $-3,13435$ & $0,01 \%$ \\
\hline ANKRD18B & 441459 & $-3,53643$ & $0,54 \%$ \\
\hline ANKRD22 & 118932 & $-1,97225$ & $0,09 \%$ \\
\hline ANKRD31 & 256006 & $-1,62531$ & $0,28 \%$ \\
\hline
\end{tabular}




\begin{tabular}{|c|c|c|c|}
\hline ANKRD44 & 91526 & 1,375263 & $0,14 \%$ \\
\hline ANKRD5 & 63926 & $-2,75407$ & $0,00 \%$ \\
\hline ANKS1B & 56899 & 2,236244 & $0,00 \%$ \\
\hline ANXA3 & 306 & $-3,68521$ & $0,00 \%$ \\
\hline ANXA6 & 309 & 2,443829 & $0,00 \%$ \\
\hline APOBECЗB & 9582 & 1,177479 & $0,77 \%$ \\
\hline APOBEC4 & 403314 & 1,222098 & $0,23 \%$ \\
\hline APOD & 347 & 1,897956 & $0,00 \%$ \\
\hline ARHGAP18 & 93663 & 1,285843 & $0,01 \%$ \\
\hline ARHGAP31 & 57514 & 1,248218 & $0,64 \%$ \\
\hline ARHGEF3 & 50650 & $-3,8971$ & $0,00 \%$ \\
\hline ARHGEF9 & 23229 & 1,095849 & $0,15 \%$ \\
\hline ARID3B & 10620 & $-2,09112$ & $0,01 \%$ \\
\hline ARL15 & 54622 & $-1,22552$ & $1,08 \%$ \\
\hline ARL4C & 10123 & $-1,3397$ & $0,87 \%$ \\
\hline ARNTL2 & 56938 & $-1,35354$ & $0,01 \%$ \\
\hline ARRDC3 & 57561 & 1,455581 & $0,02 \%$ \\
\hline ARRDC4 & 91947 & $-1,3749$ & $0,63 \%$ \\
\hline ATF7IP2 & 80063 & $-1,42218$ & $0,03 \%$ \\
\hline ATP8B1 & 5205 & 1,051258 & $0,05 \%$ \\
\hline ATXN1 & 6310 & $-1,47752$ & $0,03 \%$ \\
\hline AUTS2 & 26053 & $-1,19696$ & $0,48 \%$ \\
\hline$A X L$ & 558 & 1,292351 & $0,07 \%$ \\
\hline BAIAP2L1 & 55971 & $-2,73518$ & $0,00 \%$ \\
\hline BCL2L11 & 10018 & $-1,03777$ & $0,06 \%$ \\
\hline BGN & 633 & 2,623967 & $0,00 \%$ \\
\hline BICC1 & 80114 & 1,769613 & $0,01 \%$ \\
\hline BLMH & 642 & 1,371053 & $0,06 \%$ \\
\hline C10orf58 & 84293 & $-1,5355$ & $0,02 \%$ \\
\hline C11orf63 & 79864 & $-1,15045$ & $0,20 \%$ \\
\hline C11orf67 & 28971 & 1,65583 & $0,00 \%$ \\
\hline C14orf143 & 90141 & 1,161441 & $0,48 \%$ \\
\hline C1orf110 & 339512 & 2,254366 & $0,04 \%$ \\
\hline C1orf74 & 148304 & $-1,27157$ & $0,04 \%$ \\
\hline C20orf197 & 284756 & $-1,33579$ & $1,82 \%$ \\
\hline C2orf15 & 150590 & $-1,26495$ & $1,84 \%$ \\
\hline C4orf34 & 201895 & 1,64345 & $0,05 \%$ \\
\hline C5orf13 & 9315 & 3,298039 & $0,00 \%$ \\
\hline C5orf36 & 285600 & 1,49228 & $0,07 \%$ \\
\hline C6orf105 & 84830 & $-2,41314$ & $0,00 \%$ \\
\hline C6orf138 & 442213 & 1,089162 & $3,13 \%$ \\
\hline C6orf145 & 221749 & 1,097267 & $0,25 \%$ \\
\hline C6orf150 & 115004 & $-1,8387$ & $0,04 \%$ \\
\hline C6orf192 & 116843 & $-1,14288$ & $0,95 \%$ \\
\hline C7orf58 & 79974 & 2,049602 & $0,03 \%$ \\
\hline CACHD1 & 57685 & $-1,78617$ & $0,26 \%$ \\
\hline CAMLG & 819 & 1,246453 & $0,47 \%$ \\
\hline CATSPERB & 79820 & 1,280407 & $1,38 \%$ \\
\hline CC2D2A & 57545 & $-1,43711$ & $0,02 \%$ \\
\hline CCBE1 & 147372 & 1,563051 & $0,05 \%$ \\
\hline CCDC102B & 79839 & 2,544288 & $0,00 \%$ \\
\hline CCDC80 & 151887 & 2,113351 & $0,13 \%$ \\
\hline CD24 & 100133941 & $-6,46304$ & $0,00 \%$ \\
\hline CD2AP & 23607 & $-1,23411$ & $0,01 \%$ \\
\hline CDC42EP3 & 10602 & 1,090282 & $0,14 \%$ \\
\hline
\end{tabular}




\begin{tabular}{|c|c|c|c|}
\hline CDCP1 & 64866 & $-1,59425$ & $0,01 \%$ \\
\hline $\mathrm{CDH} 10$ & 1008 & 1,352835 & $0,04 \%$ \\
\hline $\mathrm{CDH} 18$ & 1016 & 2,817176 & $0,00 \%$ \\
\hline CDKL2 & 8999 & $-3,14481$ & $0,00 \%$ \\
\hline CDR1 & 1038 & 6,539589 & $0,00 \%$ \\
\hline $\mathrm{CHN} 1$ & 1123 & 1,586556 & $0,03 \%$ \\
\hline CHN2 & 1124 & 1,801749 & $0,00 \%$ \\
\hline CHRNB1 & 1140 & $-1,10882$ & $0,50 \%$ \\
\hline CKLF & 51192 & 1,325525 & $0,02 \%$ \\
\hline CMKLR1 & 1240 & 1,350493 & $0,22 \%$ \\
\hline CNTN3 & 5067 & 2,130302 & $0,22 \%$ \\
\hline COL17A1 & 1308 & $-1,14062$ & $0,02 \%$ \\
\hline COL8A1 & 1295 & 2,235122 & $0,06 \%$ \\
\hline COPS8 & 10920 & 1,449706 & $0,04 \%$ \\
\hline CPPED1 & 55313 & 1,491117 & $0,02 \%$ \\
\hline CPVL & 54504 & 1,452284 & $0,04 \%$ \\
\hline CSTA & 1475 & $-4,90945$ & $0,01 \%$ \\
\hline CTGF & 1490 & 1,792521 & $0,05 \%$ \\
\hline CTTNBP2 & 83992 & $-1,00108$ & $2,49 \%$ \\
\hline CWH43 & 80157 & $-2,30763$ & $0,12 \%$ \\
\hline CXorf15 & 55787 & $-1,16723$ & $0,02 \%$ \\
\hline CYBRD1 & 79901 & 1,80552 & $0,16 \%$ \\
\hline CYP4F11 & 57834 & $-1,90856$ & $0,98 \%$ \\
\hline CYR61 & 3491 & 1,859279 & $0,00 \%$ \\
\hline DARC & 2532 & 1,23437 & $0,04 \%$ \\
\hline DCLK2 & 166614 & 1,054582 & $0,92 \%$ \\
\hline DDAH1 & 23576 & 1,706363 & $0,00 \%$ \\
\hline DDX60 & 55601 & $-1,23979$ & $0,44 \%$ \\
\hline DENND2C & 163259 & $-1,44989$ & $0,04 \%$ \\
\hline DENND5B & 160518 & 1,945615 & $0,08 \%$ \\
\hline DHX32 & 55760 & $-1,19618$ & $0,05 \%$ \\
\hline DNAJB4 & 11080 & 1,392978 & $0,01 \%$ \\
\hline DNER & 92737 & 1,759985 & $0,01 \%$ \\
\hline DOCK10 & 55619 & 2,39177 & $0,01 \%$ \\
\hline DOCK4 & 9732 & 1,637033 & $0,01 \%$ \\
\hline DPYSL3 & 1809 & 1,668779 & $0,00 \%$ \\
\hline DSC3 & 1825 & $-6,00559$ & $0,00 \%$ \\
\hline DSCAM & 1826 & $-2,04186$ & $0,07 \%$ \\
\hline DSEL & 92126 & 1,448713 & $0,01 \%$ \\
\hline DSG1 & 1828 & $-2,95928$ & $0,64 \%$ \\
\hline DSG3 & 1830 & $-5,70212$ & $0,00 \%$ \\
\hline DST & 667 & 1,022589 & $0,48 \%$ \\
\hline DUSP11 & 8446 & $-1,03269$ & $0,08 \%$ \\
\hline DUSP23 & 54935 & 1,134006 & $1,23 \%$ \\
\hline EBF1 & 1879 & 1,255997 & $0,18 \%$ \\
\hline EDIL3 & 10085 & 2,205936 & $0,05 \%$ \\
\hline EFHA2 & 286097 & 1,024132 & $1,22 \%$ \\
\hline $\mathrm{EHF}$ & 26298 & $-3,37249$ & $0,04 \%$ \\
\hline ELAVL2 & 1993 & $-2,24021$ & $0,01 \%$ \\
\hline ELOVL4 & 6785 & $-1,99986$ & $0,00 \%$ \\
\hline ELOVL7 & 79993 & $-2,92231$ & $0,00 \%$ \\
\hline EMB & 133418 & $-1,1856$ & $0,27 \%$ \\
\hline EML5 & 161436 & $-1,79917$ & $0,00 \%$ \\
\hline EMP3 & 2014 & 1,335496 & $0,31 \%$ \\
\hline ENOX1 & 55068 & 1,624581 & $0,30 \%$ \\
\hline
\end{tabular}




\begin{tabular}{|c|c|c|c|}
\hline EPCAM & 4072 & $-6,65207$ & $0,00 \%$ \\
\hline EPHX4 & 253152 & $-1,16738$ & $2,39 \%$ \\
\hline EPS8 & 2059 & 1,703254 & $0,00 \%$ \\
\hline ERGIC1 & 57222 & 1,002524 & $4,90 \%$ \\
\hline ESRP1 & 54845 & $-5,5253$ & $0,00 \%$ \\
\hline ESRP2 & 80004 & $-1,18459$ & $1,84 \%$ \\
\hline ETV1 & 2115 & 1,144286 & $0,28 \%$ \\
\hline EXPH5 & 23086 & $-2,22089$ & $0,01 \%$ \\
\hline FAM114A2 & 10827 & 1,053817 & $0,19 \%$ \\
\hline FAM117B & 150864 & $-1,34213$ & $1,12 \%$ \\
\hline FAM135A & 57579 & $-1,40498$ & $0,09 \%$ \\
\hline FAM160A1 & 729830 & $-1,35765$ & $2,72 \%$ \\
\hline FAM169A & 26049 & $-3,06387$ & $0,00 \%$ \\
\hline FAM171B & 165215 & $-1,25641$ & $1,91 \%$ \\
\hline FAM172A & 83989 & 1,196932 & $0,43 \%$ \\
\hline FAM173B & 134145 & 1,019644 & $0,41 \%$ \\
\hline FAM60A & 58516 & $-1,23921$ & $0,06 \%$ \\
\hline FAM65B & 9750 & 2,510136 & $0,00 \%$ \\
\hline FAM69A & 388650 & $-1,8623$ & $0,05 \%$ \\
\hline FAM83A & 84985 & $-1,33845$ & $0,01 \%$ \\
\hline FAM83B & 222584 & $-5,88642$ & $0,00 \%$ \\
\hline FAM84B & 157638 & $-1,70246$ & $0,18 \%$ \\
\hline FAM91A1 & 157769 & $-1,77283$ & $0,00 \%$ \\
\hline FANK1 & 92565 & $-1,26586$ & $0,34 \%$ \\
\hline FAT2 & 2196 & $-2,5925$ & $0,00 \%$ \\
\hline FAT4 & 79633 & 2,619804 & $0,00 \%$ \\
\hline FBLN1 & 2192 & 2,454517 & $0,00 \%$ \\
\hline FBLN5 & 10516 & 6,701929 & $0,00 \%$ \\
\hline FBN1 & 2200 & 4,611196 & $0,00 \%$ \\
\hline FBN2 & 2201 & 3,058033 & $0,00 \%$ \\
\hline FBXL14 & 144699 & 1,002116 & $2,19 \%$ \\
\hline FERMT1 & 55612 & $-1,87064$ & $0,01 \%$ \\
\hline FERMT2 & 10979 & 1,845866 & $0,01 \%$ \\
\hline FEZ1 & 9638 & $-2,48128$ & $0,02 \%$ \\
\hline FGD6 & 55785 & $-1,52464$ & $0,02 \%$ \\
\hline FGFBP1 & 9982 & $-5,06542$ & $0,00 \%$ \\
\hline FHL1 & 2273 & 1,050759 & $2,37 \%$ \\
\hline FHOD3 & 80206 & $-2,15138$ & $0,06 \%$ \\
\hline FIGNL1 & 63979 & 1,29488 & $0,03 \%$ \\
\hline FKBP5 & 2289 & 1,326128 & $0,01 \%$ \\
\hline FKBP7 & 51661 & 1,685212 & $0,02 \%$ \\
\hline FLJ32810 & 143872 & $-1,34337$ & $0,05 \%$ \\
\hline FLVCR2 & 55640 & 2,071016 & $0,01 \%$ \\
\hline FOXN3 & 1112 & 2,245154 & $0,02 \%$ \\
\hline FRAS1 & 80144 & $-1,44666$ & $0,06 \%$ \\
\hline FRK & 2444 & $-2,70206$ & $0,00 \%$ \\
\hline FRMD3 & 257019 & 1,406831 & $0,08 \%$ \\
\hline FRMD4B & 23150 & $-3,18408$ & $0,01 \%$ \\
\hline FRMD6 & 122786 & 1,197235 & $0,09 \%$ \\
\hline FRRS1 & 391059 & $-3,91441$ & $0,00 \%$ \\
\hline FSTL1 & 11167 & 1,0537 & $0,25 \%$ \\
\hline FTO & 79068 & 1,034197 & $0,18 \%$ \\
\hline FUNDC1 & 139341 & $-1,02094$ & $0,52 \%$ \\
\hline GBP6 & 163351 & $-1,49148$ & $1,72 \%$ \\
\hline GCA & 25801 & $-1,72873$ & $2,55 \%$ \\
\hline
\end{tabular}




\begin{tabular}{|c|c|c|c|}
\hline GDPD3 & 79153 & $-1,70309$ & $3,09 \%$ \\
\hline GJB2 & 2706 & $-2,49628$ & $0,00 \%$ \\
\hline GJB5 & 2709 & $-1,03876$ & $0,85 \%$ \\
\hline GLCCl1 & 113263 & 2,00416 & $0,01 \%$ \\
\hline GLRX & 2745 & 3,127223 & $0,00 \%$ \\
\hline GLT8D2 & 83468 & $-1,31767$ & $4,65 \%$ \\
\hline GLYATL2 & 219970 & 2,165953 & $0,01 \%$ \\
\hline GPR110 & 266977 & $-2,90007$ & $0,04 \%$ \\
\hline GPR115 & 221393 & $-1,52097$ & $1,12 \%$ \\
\hline GPR125 & 166647 & $-1,22743$ & $0,06 \%$ \\
\hline GPR56 & 9289 & $-1,02971$ & $1,78 \%$ \\
\hline GPR63 & 81491 & $-1,19755$ & $0,24 \%$ \\
\hline GPR64 & 10149 & 2,391411 & $0,06 \%$ \\
\hline GPR87 & 53836 & $-2,90587$ & $0,01 \%$ \\
\hline GRB14 & 2888 & $-3,10521$ & $0,05 \%$ \\
\hline GREB1L & 80000 & 1,355142 & $0,88 \%$ \\
\hline GREM1 & 26585 & 3,508672 & $0,00 \%$ \\
\hline GRHL1 & 29841 & $-1,13139$ & $0,45 \%$ \\
\hline GRHL2 & 79977 & $-4,17071$ & $0,00 \%$ \\
\hline GRIP1 & 23426 & $-1,75377$ & $0,04 \%$ \\
\hline GULP1 & 51454 & $-4,15256$ & $0,00 \%$ \\
\hline HAS2 & 3037 & 3,726369 & $0,00 \%$ \\
\hline HDX & 139324 & $-1,01034$ & $0,39 \%$ \\
\hline HELB & 92797 & $-1,11896$ & $0,21 \%$ \\
\hline HEPHL1 & 341208 & $-1,4025$ & $0,02 \%$ \\
\hline HERC5 & 51191 & $-1,33395$ & $0,26 \%$ \\
\hline HERC6 & 55008 & $-2,75702$ & $0,00 \%$ \\
\hline HIPK2 & 28996 & 1,031728 & $0,12 \%$ \\
\hline HIVEP1 & 3096 & $-1,0983$ & $0,09 \%$ \\
\hline HIVEP2 & 3097 & $-1,24656$ & $0,04 \%$ \\
\hline HOMER1 & 9456 & $-1,9642$ & $0,02 \%$ \\
\hline HOMER2 & 9455 & $-1,07916$ & $0,50 \%$ \\
\hline HOOK1 & 51361 & $-4,9158$ & $0,00 \%$ \\
\hline HPGD & 3248 & 1,850578 & $0,00 \%$ \\
\hline HSD17B11 & 51170 & 1,231886 & $0,16 \%$ \\
\hline HSPC159 & 29094 & $-1,21808$ & $0,48 \%$ \\
\hline IER3 & 8870 & $-1,12068$ & $1,07 \%$ \\
\hline IFI44 & 10561 & $-1,33467$ & $0,39 \%$ \\
\hline IFI44L & 10964 & 1,974194 & $0,24 \%$ \\
\hline IGFBP4 & 3487 & 1,715999 & $0,11 \%$ \\
\hline IKZF2 & 22807 & 1,348883 & $0,09 \%$ \\
\hline IL1RN & 3557 & $-1,09806$ & $2,42 \%$ \\
\hline INSC & 387755 & 1,394635 & $0,10 \%$ \\
\hline IQCA1 & 79781 & $-1,49016$ & $0,25 \%$ \\
\hline IRF6 & 3664 & $-2,66252$ & $0,00 \%$ \\
\hline ITGBL1 & 9358 & 3,796898 & $0,00 \%$ \\
\hline JAKMIP2 & 9832 & 3,973465 & $0,00 \%$ \\
\hline JHDM1D & 80853 & $-1,25011$ & $0,32 \%$ \\
\hline JMY & 133746 & $-1,09341$ & $0,09 \%$ \\
\hline JPH1 & 56704 & $-1,68337$ & $0,11 \%$ \\
\hline KCNJ15 & 3772 & $-1,79119$ & $0,19 \%$ \\
\hline KCNJ6 & 3763 & 2,850055 & $0,00 \%$ \\
\hline KCNK1 & 3775 & $-1,05598$ & $3,05 \%$ \\
\hline KCNK2 & 3776 & 1,780088 & $0,02 \%$ \\
\hline KCTD1 & 284252 & $-1,52502$ & $0,06 \%$ \\
\hline
\end{tabular}




\begin{tabular}{|c|c|c|c|}
\hline KDELC2 & 143888 & 1,667794 & $0,00 \%$ \\
\hline KDM5B & 10765 & $-1,23665$ & $0,56 \%$ \\
\hline KDM6A & 7403 & $-1,09752$ & $0,02 \%$ \\
\hline KIAA0040 & 9674 & $-2,86611$ & $0,01 \%$ \\
\hline KIAA0196 & 9897 & $-1,23827$ & $0,01 \%$ \\
\hline KIAA1107 & 23285 & $-1,05033$ & $3,83 \%$ \\
\hline KIAA1217 & 56243 & $-1,62161$ & $0,02 \%$ \\
\hline KIF13A & 63971 & $-1,45175$ & $0,03 \%$ \\
\hline KIF20A & 10112 & 1,341971 & $0,25 \%$ \\
\hline KLF12 & 11278 & 1,103155 & $0,04 \%$ \\
\hline KLF5 & 688 & $-1,07177$ & $0,14 \%$ \\
\hline KLHL4 & 56062 & 2,846087 & $0,00 \%$ \\
\hline KLK5 & 25818 & $-1,10645$ & $0,27 \%$ \\
\hline KRCC1 & 51315 & $-1,1865$ & $0,11 \%$ \\
\hline KRT14 & 3861 & $-2,00059$ & $0,69 \%$ \\
\hline KRT15 & 3866 & $-1,63253$ & $0,01 \%$ \\
\hline KRT17 & 3872 & $-1,71116$ & $0,35 \%$ \\
\hline KRT5 & 3852 & $-2,6302$ & $0,09 \%$ \\
\hline KRT6A & 3853 & $-7,37797$ & $0,00 \%$ \\
\hline KRT6B & 3854 & $-3,57352$ & $0,02 \%$ \\
\hline KRT6C & 286887 & $-3,76575$ & $0,09 \%$ \\
\hline KRT8 & 3856 & $-1,56693$ & $0,33 \%$ \\
\hline LAD1 & 3898 & $-1,0365$ & $0,94 \%$ \\
\hline LCP1 & 3936 & $-2,38467$ & $0,00 \%$ \\
\hline LEPREL1 & 55214 & $-2,50432$ & $0,04 \%$ \\
\hline LGR4 & 55366 & $-1,59771$ & $0,01 \%$ \\
\hline LIMA1 & 51474 & $-1,56774$ & $0,01 \%$ \\
\hline LMNB1 & 4001 & 1,130161 & $0,06 \%$ \\
\hline LOC100130348 & 100130348 & 1,067129 & $0,76 \%$ \\
\hline LOC100131726 & 100131726 & $-2,47355$ & $0,00 \%$ \\
\hline LOC100288413 & 100288413 & $-2,93297$ & $0,00 \%$ \\
\hline LOC100288461 & 100288461 & $-1,64251$ & $0,32 \%$ \\
\hline LOC554202 & 554202 & 1,200015 & $1,45 \%$ \\
\hline LOC642587 & 642587 & $-1,18691$ & $1,21 \%$ \\
\hline LOX & 4015 & 3,786601 & $0,00 \%$ \\
\hline LPHN2 & 23266 & $-5,67536$ & $0,00 \%$ \\
\hline LPHN3 & 23284 & 3,301966 & $0,00 \%$ \\
\hline LRP12 & 29967 & $-1,1913$ & $0,92 \%$ \\
\hline LRRC1 & 55227 & $-1,71383$ & $0,03 \%$ \\
\hline LRRC16A & 55604 & $-1,51283$ & $0,05 \%$ \\
\hline LTBP2 & 4053 & 1,18168 & $0,03 \%$ \\
\hline MACC1 & 346389 & $-4,4822$ & $0,00 \%$ \\
\hline MAL2 & 114569 & $-7,14065$ & $0,00 \%$ \\
\hline MAMLD1 & 10046 & 1,145021 & $0,95 \%$ \\
\hline MAP1B & 4131 & 4,872148 & $0,00 \%$ \\
\hline MAP1LC3B2 & 643246 & $-1,08572$ & $4,22 \%$ \\
\hline MAP7 & 9053 & $-2,73642$ & $0,02 \%$ \\
\hline MAP9 & 79884 & $-2,18213$ & $0,01 \%$ \\
\hline MARCH3 & 115123 & 1,509304 & $0,01 \%$ \\
\hline MARK1 & 4139 & $-1,77822$ & $0,01 \%$ \\
\hline MARVELD2 & 153562 & $-3,22842$ & $0,00 \%$ \\
\hline MBNL2 & 10150 & $-1,41103$ & $0,07 \%$ \\
\hline MCOLN2 & 255231 & $-2,28053$ & $0,03 \%$ \\
\hline MCOLN3 & 55283 & $-3,08733$ & $0,00 \%$ \\
\hline MCTP1 & 79772 & 2,610116 & $0,00 \%$ \\
\hline
\end{tabular}




\begin{tabular}{|c|c|c|c|}
\hline MCTP2 & 55784 & $-1,62154$ & $0,11 \%$ \\
\hline MED10 & 84246 & 1,095471 & $0,39 \%$ \\
\hline MED14 & 9282 & $-1,11691$ & $0,03 \%$ \\
\hline MED30 & 90390 & $-1,07339$ & $0,45 \%$ \\
\hline MED7 & 9443 & 1,123571 & $0,52 \%$ \\
\hline MEST & 4232 & $-1,01691$ & $0,72 \%$ \\
\hline MFAP5 & 8076 & $-3,40629$ & $0,01 \%$ \\
\hline MGP & 4256 & 2,070706 & $4,29 \%$ \\
\hline MICALCL & 84953 & $-1,65315$ & $0,04 \%$ \\
\hline MIOS & 54468 & 1,846857 & $0,00 \%$ \\
\hline MLLT3 & 4300 & 1,555401 & $0,11 \%$ \\
\hline MLPH & 79083 & 1,372648 & $0,27 \%$ \\
\hline MMP16 & 4325 & 1,270034 & $0,38 \%$ \\
\hline MMP19 & 4327 & 1,502709 & $0,07 \%$ \\
\hline MOBKL2B & 79817 & $-1,84582$ & $0,47 \%$ \\
\hline MOSC1 & 64757 & $-2,13433$ & $0,00 \%$ \\
\hline MOXD1 & 26002 & 1,184718 & $1,37 \%$ \\
\hline MPZL2 & 10205 & $-4,23682$ & $0,07 \%$ \\
\hline MPZL3 & 196264 & $-1,27623$ & $0,05 \%$ \\
\hline MREG & 55686 & $-1,82235$ & $0,01 \%$ \\
\hline MSRB3 & 253827 & 1,115227 & $1,93 \%$ \\
\hline MT1E & 4493 & 1,569602 & $0,04 \%$ \\
\hline MT1L & 4500 & 1,12916 & $0,55 \%$ \\
\hline MT1X & 4501 & 1,027699 & $0,68 \%$ \\
\hline MTSS1 & 9788 & $-2,40624$ & $0,00 \%$ \\
\hline MTUS1 & 57509 & $-2,00517$ & $0,00 \%$ \\
\hline MY01D & 4642 & $-3,07542$ & $0,00 \%$ \\
\hline MY01E & 4643 & $-1,29157$ & $0,02 \%$ \\
\hline MYO5B & 4645 & $-2,45463$ & $0,01 \%$ \\
\hline MYO5C & 55930 & $-1,3238$ & $0,11 \%$ \\
\hline MYO6 & 4646 & $-2,01269$ & $0,04 \%$ \\
\hline MYPN & 84665 & 3,007115 & $0,01 \%$ \\
\hline N4BP2 & 55728 & $-1,08137$ & $0,12 \%$ \\
\hline NAV1 & 89796 & 1,370974 & $0,47 \%$ \\
\hline NAV2 & 89797 & 2,374518 & $0,00 \%$ \\
\hline NCEH1 & 57552 & 1,159076 & $0,03 \%$ \\
\hline NCRNA00052 & 145978 & $-1,2509$ & $0,27 \%$ \\
\hline NDFIP2 & 54602 & $-1,14856$ & $0,08 \%$ \\
\hline NEBL & 10529 & 1,991245 & $0,00 \%$ \\
\hline NETO1 & 81832 & 4,563266 & $0,00 \%$ \\
\hline NID1 & 4811 & 2,349584 & $0,00 \%$ \\
\hline NIPAL1 & 152519 & $-1,41235$ & $0,03 \%$ \\
\hline NKAIN2 & 154215 & 3,893879 & $0,00 \%$ \\
\hline NPNT & 255743 & $-1,17971$ & $0,04 \%$ \\
\hline NQO1 & 1728 & 1,423442 & $0,03 \%$ \\
\hline NR2F1 & 7025 & 1,006493 & $1,48 \%$ \\
\hline NRIP1 & 8204 & $-1,47093$ & $0,04 \%$ \\
\hline NRP2 & 8828 & $-1,42078$ & $0,02 \%$ \\
\hline NUDT11 & 55190 & $-2,26086$ & $0,03 \%$ \\
\hline NUP62CL & 54830 & $-1,07661$ & $0,55 \%$ \\
\hline OCIAD2 & 132299 & $-1,46789$ & $4,80 \%$ \\
\hline OGFRL1 & 79627 & 1,090624 & $0,15 \%$ \\
\hline OMD & 4958 & 1,080998 & $0,99 \%$ \\
\hline OPN3 & 23596 & 1,005271 & $0,91 \%$ \\
\hline OVCH1 & 341350 & 1,508822 & $0,11 \%$ \\
\hline
\end{tabular}




\begin{tabular}{|c|c|c|c|}
\hline PAG1 & 55824 & 1,566696 & $0,04 \%$ \\
\hline PAM & 5066 & 1,523384 & $0,02 \%$ \\
\hline PARD3B & 117583 & 1,196151 & $0,59 \%$ \\
\hline PCDH9 & 5101 & 1,243515 & $0,09 \%$ \\
\hline PCDHGB4 & 8641 & 1,859413 & $0,16 \%$ \\
\hline PCDHGB5 & 56101 & 1,501365 & $0,19 \%$ \\
\hline PCDHGB7 & 56099 & 1,090372 & $0,06 \%$ \\
\hline PCOLCE & 5118 & 1,041778 & $0,08 \%$ \\
\hline PDPN & 10630 & $-2,03677$ & $0,00 \%$ \\
\hline PGBD3 & 267004 & 1,893771 & $0,00 \%$ \\
\hline PHF15 & 23338 & 1,204314 & $0,38 \%$ \\
\hline PHLDB2 & 90102 & $-1,11584$ & $0,04 \%$ \\
\hline PI3 & 5266 & $-1,22531$ & $4,05 \%$ \\
\hline PLA2R1 & 22925 & 1,810461 & $0,11 \%$ \\
\hline PLD5 & 200150 & $-3,80382$ & $0,07 \%$ \\
\hline PLEKHA5 & 54477 & $-1,29778$ & $0,03 \%$ \\
\hline PLEKHA7 & 144100 & $-1,1124$ & $0,07 \%$ \\
\hline PLS1 & 5357 & $-1,23883$ & $0,01 \%$ \\
\hline PLSCR4 & 57088 & 1,484274 & $0,03 \%$ \\
\hline PLXDC2 & 84898 & 4,076799 & $0,00 \%$ \\
\hline PMFBP1 & 83449 & 1,14764 & $4,74 \%$ \\
\hline PMP22 & 5376 & 2,05257 & $0,01 \%$ \\
\hline PNLIPRP3 & 119548 & $-3,39355$ & $0,02 \%$ \\
\hline POF1B & 79983 & $-1,99642$ & $0,73 \%$ \\
\hline POSTN & 10631 & 3,884249 & $0,00 \%$ \\
\hline PPP1R1C & 151242 & $-1,30708$ & $0,21 \%$ \\
\hline PPP4R4 & 57718 & $-1,7792$ & $0,38 \%$ \\
\hline PRDM1 & 639 & $-3,07636$ & $0,08 \%$ \\
\hline PRKD1 & 5587 & 4,833212 & $0,00 \%$ \\
\hline PRR16 & 51334 & 4,013681 & $0,00 \%$ \\
\hline PRRG4 & 79056 & $-2,40412$ & $0,06 \%$ \\
\hline PRRX1 & 5396 & 4,044821 & $0,00 \%$ \\
\hline PRSS23 & 11098 & 1,701233 & $0,28 \%$ \\
\hline PRTFDC1 & 56952 & 1,2804 & $0,55 \%$ \\
\hline PRUNE2 & 158471 & 3,104814 & $0,00 \%$ \\
\hline PSTPIP2 & 9050 & $-3,98872$ & $0,01 \%$ \\
\hline PTGFRN & 5738 & $-2,78819$ & $0,00 \%$ \\
\hline PTHLH & 5744 & $-1,5582$ & $0,27 \%$ \\
\hline PTPN13 & 5783 & $-1,5903$ & $0,01 \%$ \\
\hline PTPN3 & 5774 & $-1,25408$ & $0,09 \%$ \\
\hline PTX3 & 5806 & 3,111522 & $0,01 \%$ \\
\hline PYGO1 & 26108 & 1,020611 & $0,14 \%$ \\
\hline QPCT & 25797 & $-1,32474$ & $2,87 \%$ \\
\hline RAB25 & 57111 & $-3,06086$ & $0,04 \%$ \\
\hline RAB38 & 23682 & $-1,9442$ & $0,04 \%$ \\
\hline RAB9A & 9367 & $-1,05109$ & $0,17 \%$ \\
\hline RAPGEF5 & 9771 & $-1,85171$ & $0,03 \%$ \\
\hline RASA3 & 22821 & 1,03946 & $0,58 \%$ \\
\hline RASEF & 158158 & $-1,92983$ & $0,36 \%$ \\
\hline RASSF6 & 166824 & $-2,26082$ & $0,02 \%$ \\
\hline RASSF9 & 9182 & $-2,3504$ & $0,02 \%$ \\
\hline RAVER2 & 55225 & $-1,10708$ & $0,35 \%$ \\
\hline REPS2 & 9185 & 3,155223 & $0,00 \%$ \\
\hline RG9MTD2 & 93587 & $-1,02264$ & $0,35 \%$ \\
\hline RGL1 & 23179 & 2,164084 & $0,00 \%$ \\
\hline
\end{tabular}




\begin{tabular}{|c|c|c|c|}
\hline RGS17 & 26575 & 1,193645 & $0,13 \%$ \\
\hline RGS4 & 5999 & 3,197985 & $0,00 \%$ \\
\hline RGS7 & 6000 & 3,049249 & $0,00 \%$ \\
\hline RHOBTB3 & 22836 & 4,931677 & $0,00 \%$ \\
\hline RIMS2 & 9699 & $-2,90152$ & $0,01 \%$ \\
\hline RMND5A & 64795 & $-1,12968$ & $0,02 \%$ \\
\hline RND3 & 390 & $-1,39334$ & $1,12 \%$ \\
\hline RNF128 & 79589 & $-1,01587$ & $0,94 \%$ \\
\hline RNF144B & 255488 & $-1,18984$ & $0,12 \%$ \\
\hline ROR1 & 4919 & 1,255677 & $0,05 \%$ \\
\hline ROS1 & 6098 & 4,896899 & $0,00 \%$ \\
\hline RTN1 & 6252 & 4,491045 & $0,00 \%$ \\
\hline S100A14 & 57402 & $-2,62316$ & $1,07 \%$ \\
\hline S100A2 & 6273 & $-2,05778$ & $0,16 \%$ \\
\hline S100A8 & 6279 & $-3,32892$ & $0,27 \%$ \\
\hline SAA1 & 6288 & $-2,95641$ & $0,19 \%$ \\
\hline SAMD12 & 401474 & $-1,58486$ & $1,10 \%$ \\
\hline SATB2 & 23314 & 1,006965 & $0,42 \%$ \\
\hline SAV1 & 60485 & $-1,42979$ & $0,01 \%$ \\
\hline SCCPDH & 51097 & 1,029792 & $0,18 \%$ \\
\hline SCEL & 8796 & $-2,04428$ & $0,60 \%$ \\
\hline SCML2 & 10389 & 1,601303 & $0,42 \%$ \\
\hline SDR42E1 & 93517 & $-1,21875$ & $0,25 \%$ \\
\hline SEL1L3 & 23231 & $-1,6722$ & $0,08 \%$ \\
\hline SERPINB1 & 1992 & $-2,68356$ & $0,02 \%$ \\
\hline SERPINB13 & 5275 & $-4,09617$ & $0,00 \%$ \\
\hline SERPINB2 & 5055 & $-5,96889$ & $0,00 \%$ \\
\hline SERPINB7 & 8710 & $-1,32738$ & $0,01 \%$ \\
\hline SERPINE2 & 5270 & 1,202091 & $0,52 \%$ \\
\hline SH3BGRL2 & 83699 & $-3,36119$ & $0,03 \%$ \\
\hline SH3PXD2A & 9644 & $-1,1057$ & $0,32 \%$ \\
\hline SH3RF2 & 153769 & $-1,39784$ & $0,25 \%$ \\
\hline SH3YL1 & 26751 & $-1,01398$ & $0,61 \%$ \\
\hline SHISA9 & 729993 & 1,396862 & $0,04 \%$ \\
\hline SHROOM3 & 57619 & $-1,2655$ & $0,03 \%$ \\
\hline SLC12A8 & 84561 & $-1,14814$ & $0,18 \%$ \\
\hline SLC16A7 & 9194 & 1,539421 & $0,35 \%$ \\
\hline SLC1A3 & 6507 & $-3,76094$ & $0,00 \%$ \\
\hline SLC22A4 & 6583 & 2,257355 & $0,00 \%$ \\
\hline SLC22A5 & 6584 & 1,12614 & $0,38 \%$ \\
\hline SLC26A2 & 1836 & 1,969695 & $0,01 \%$ \\
\hline SLC2A12 & 154091 & $-1,02733$ & $0,96 \%$ \\
\hline SLC38A4 & 55089 & 2,566578 & $0,07 \%$ \\
\hline SLC38A6 & 145389 & 1,420855 & $0,01 \%$ \\
\hline SLC39A8 & 64116 & $-1,58542$ & $0,02 \%$ \\
\hline SLC3A2 & 6520 & $-1,31768$ & $0,02 \%$ \\
\hline SLC41A2 & 84102 & $-2,07933$ & $0,05 \%$ \\
\hline SLC7A11 & 23657 & $-1,12555$ & $0,21 \%$ \\
\hline SLC7A2 & 6542 & 2,33479 & $0,00 \%$ \\
\hline SLC7A5 & 8140 & $-2,67683$ & $0,00 \%$ \\
\hline SLC7A8 & 23428 & $-2,29589$ & $0,01 \%$ \\
\hline SLFN11 & 91607 & 1,795829 & $0,04 \%$ \\
\hline SLITRK6 & 84189 & $-2,44183$ & $0,14 \%$ \\
\hline SNAPC1 & 6617 & 1,271351 & $0,03 \%$ \\
\hline SNORD113-3 & 767563 & 1,800731 & $0,00 \%$ \\
\hline
\end{tabular}




\begin{tabular}{|c|c|c|c|}
\hline SNORD114-2 & 767578 & 2,549792 & $0,03 \%$ \\
\hline SNX10 & 29887 & $-4,05354$ & $0,00 \%$ \\
\hline SNX16 & 64089 & $-1,01672$ & $0,18 \%$ \\
\hline SNX9 & 51429 & $-1,1072$ & $0,13 \%$ \\
\hline SPAG1 & 6674 & $-1,90708$ & $0,01 \%$ \\
\hline SPARC & 6678 & 1,68683 & $0,02 \%$ \\
\hline SPATA6 & 54558 & 2,014945 & $0,01 \%$ \\
\hline SPINT2 & 10653 & $-1,30862$ & $0,03 \%$ \\
\hline SPRR1A & 6698 & $-3,29695$ & $0,12 \%$ \\
\hline SPRR1B & 6699 & $-5,36692$ & $0,00 \%$ \\
\hline SPRR2A & 6700 & $-2,54372$ & $0,67 \%$ \\
\hline SPRR2D & 6703 & $-3,67265$ & $0,04 \%$ \\
\hline SRGN & 5552 & 5,083677 & $0,00 \%$ \\
\hline SRPX & 8406 & 1,011377 & $0,40 \%$ \\
\hline SSBP2 & 23635 & 1,146886 & $0,05 \%$ \\
\hline ST6GALNAC2 & 10610 & $-1,56438$ & $0,01 \%$ \\
\hline STARD13 & 90627 & 1,406931 & $0,04 \%$ \\
\hline STARD4 & 134429 & 1,083505 & $0,19 \%$ \\
\hline STC2 & 8614 & 1,828905 & $0,00 \%$ \\
\hline STEAP2 & 261729 & $-1,29081$ & $0,04 \%$ \\
\hline STK17A & 9263 & $-1,05587$ & $0,03 \%$ \\
\hline STRBP & 55342 & $-1,24113$ & $0,02 \%$ \\
\hline SULF2 & 55959 & $-1,6272$ & $0,67 \%$ \\
\hline SULT1B1 & 27284 & 1,982786 & $0,03 \%$ \\
\hline SVEP1 & 79987 & 1,452688 & $0,05 \%$ \\
\hline TACSTD2 & 4070 & $-1,81344$ & $0,15 \%$ \\
\hline TBC1D30 & 23329 & $-1,23731$ & $0,42 \%$ \\
\hline TBX18 & 9096 & 3,266421 & $0,00 \%$ \\
\hline TCF4 & 6925 & $-1,3216$ & $0,25 \%$ \\
\hline TERT & 7015 & $-1,29777$ & $0,27 \%$ \\
\hline TES & 26136 & $-1,42624$ & $0,03 \%$ \\
\hline TFCP2L1 & 29842 & $-1,84987$ & $0,01 \%$ \\
\hline TFPI2 & 7980 & $-1,21006$ & $0,56 \%$ \\
\hline TGFBR3 & 7049 & 1,742554 & $0,03 \%$ \\
\hline TIMP3 & 7078 & $-1,8301$ & $0,07 \%$ \\
\hline TLL1 & 7092 & $-2,77388$ & $0,00 \%$ \\
\hline TM4SF1 & 4071 & 1,318496 & $0,36 \%$ \\
\hline TMC5 & 79838 & $-1,0131$ & $4,24 \%$ \\
\hline TMEM117 & 84216 & $-3,20833$ & $0,02 \%$ \\
\hline TMEM136 & 219902 & 1,09539 & $0,29 \%$ \\
\hline TMEM154 & 201799 & $-1,76969$ & $0,11 \%$ \\
\hline TMEM156 & 80008 & 2,068505 & $0,02 \%$ \\
\hline TMEM171 & 134285 & $-1,18685$ & $0,14 \%$ \\
\hline TMEM20 & 159371 & $-1,03944$ & $0,19 \%$ \\
\hline TMEM200A & 114801 & $-1,42492$ & $0,10 \%$ \\
\hline TMEM30B & 161291 & $-1,68786$ & $0,08 \%$ \\
\hline TMEM40 & 55287 & $-1,35076$ & $0,20 \%$ \\
\hline TMEM47 & 83604 & 1,010084 & $0,08 \%$ \\
\hline TMEM56 & 148534 & $-1,95348$ & $0,03 \%$ \\
\hline TMEM65 & 157378 & $-1,30271$ & $0,22 \%$ \\
\hline TMEM97 & 27346 & 1,128033 & $0,08 \%$ \\
\hline TMTC1 & 83857 & 2,774732 & $0,02 \%$ \\
\hline TMTC2 & 160335 & $-1,23712$ & $0,07 \%$ \\
\hline TNFAIP8L3 & 388121 & 1,157809 & $0,80 \%$ \\
\hline TNIK & 23043 & $-1,74508$ & $0,00 \%$ \\
\hline
\end{tabular}




\begin{tabular}{|c|c|c|c|}
\hline TNXA & 7146 & $-1,21702$ & $1,43 \%$ \\
\hline TOX & 9760 & 3,233164 & $0,00 \%$ \\
\hline TP63 & 8626 & $-4,94493$ & $0,00 \%$ \\
\hline TPD52 & 7163 & $-1,58528$ & $0,03 \%$ \\
\hline TPD52L1 & 7164 & $-3,33346$ & $0,00 \%$ \\
\hline TRAF3IP3 & 80342 & $-1,32956$ & $0,30 \%$ \\
\hline TRAK2 & 66008 & 1,114379 & $0,43 \%$ \\
\hline TRAM2 & 9697 & 2,069473 & $0,00 \%$ \\
\hline TRBV20-1 & 28567 & 1,675265 & $0,35 \%$ \\
\hline TRHDE & 29953 & $-2,15256$ & $0,22 \%$ \\
\hline TRIB1 & 10221 & $-1,65715$ & $0,01 \%$ \\
\hline TRIM29 & 23650 & $-1,20896$ & $0,63 \%$ \\
\hline TRIML2 & 205860 & $-1,11224$ & $0,59 \%$ \\
\hline TRPM3 & 80036 & 2,603398 & $0,00 \%$ \\
\hline TSGA10 & 80705 & $-1,14918$ & $0,35 \%$ \\
\hline TSHZ2 & 128553 & $-1,21223$ & $0,28 \%$ \\
\hline TSPAN1 & 10103 & $-2,10687$ & $0,21 \%$ \\
\hline TSPYL2 & 64061 & 1,151877 & $0,27 \%$ \\
\hline TTC35 & 9694 & $-1,11323$ & $0,80 \%$ \\
\hline TXNDC15 & 79770 & 1,355917 & $0,22 \%$ \\
\hline TXNIP & 10628 & 2,972929 & $0,02 \%$ \\
\hline UCA1 & 652995 & $-4,93334$ & $0,00 \%$ \\
\hline USP53 & 54532 & $-1,50934$ & $0,40 \%$ \\
\hline UTRN & 7402 & 1,002226 & $0,20 \%$ \\
\hline VGLL1 & 51442 & $-1,80911$ & $0,36 \%$ \\
\hline VGLL3 & 389136 & $-1,4202$ & $0,07 \%$ \\
\hline VIM & 7431 & 1,909232 & $0,18 \%$ \\
\hline VSNL1 & 7447 & $-3,85166$ & $0,01 \%$ \\
\hline WDR17 & 116966 & $-1,80561$ & $0,17 \%$ \\
\hline WDR67 & 93594 & $-1,16269$ & $0,05 \%$ \\
\hline WDYHV1 & 55093 & $-1,05481$ & $0,19 \%$ \\
\hline WIPF1 & 7456 & 1,237134 & $0,22 \%$ \\
\hline WIPI1 & 55062 & 1,196059 & $2,32 \%$ \\
\hline WISP3 & 8838 & $-1,79233$ & $0,02 \%$ \\
\hline WWC1 & 23286 & $-1,55353$ & $0,53 \%$ \\
\hline ZBED2 & 79413 & $-2,08618$ & $0,03 \%$ \\
\hline ZBTB38 & 253461 & 1,196229 & $0,02 \%$ \\
\hline ZEB1 & 6935 & 4,307825 & $0,00 \%$ \\
\hline ZEB2 & 9839 & 3,018026 & $0,00 \%$ \\
\hline $\mathrm{ZHX1}$ & 11244 & $-1,44933$ & $0,01 \%$ \\
\hline ZHX2 & 22882 & $-1,41799$ & $0,02 \%$ \\
\hline ZHX3 & 23051 & 1,14019 & $0,03 \%$ \\
\hline ZMYND8 & 23613 & 1,555456 & $0,01 \%$ \\
\hline ZNF114 & 163071 & $-2,67296$ & $0,00 \%$ \\
\hline ZNF160 & 90338 & 1,084365 & $0,05 \%$ \\
\hline ZNF165 & 7718 & $-1,43226$ & $0,25 \%$ \\
\hline ZNF204P & 7754 & $-2,51143$ & $1,51 \%$ \\
\hline ZNF22 & 7570 & 1,078539 & $0,05 \%$ \\
\hline ZNF253 & 56242 & 1,375441 & $0,38 \%$ \\
\hline ZNF287 & 57336 & 1,0561 & $0,11 \%$ \\
\hline ZNF429 & 353088 & 1,160922 & $0,19 \%$ \\
\hline ZNF43 & 7594 & 1,76211 & $0,83 \%$ \\
\hline ZNF441 & 126068 & 1,584626 & $0,01 \%$ \\
\hline ZNF506 & 440515 & 1,080101 & $0,29 \%$ \\
\hline ZNF528 & 84436 & 2,142157 & $0,01 \%$ \\
\hline
\end{tabular}




\begin{tabular}{llrl}
\hline ZNF558 & 148156 & 2,389652 & $0,02 \%$ \\
ZNF618 & 114991 & $-1,1591$ & $0,12 \%$ \\
ZNF750 & 79755 & $-1,21991$ & $2,17 \%$ \\
ZNF799 & 90576 & 1,353518 & $0,36 \%$ \\
ZNF93 & 81931 & 1,303863 & $0,28 \%$ \\
ZXDB & 158586 & 1,250177 & $0,02 \%$
\end{tabular}

\section{Salinomycin: Survival assay}

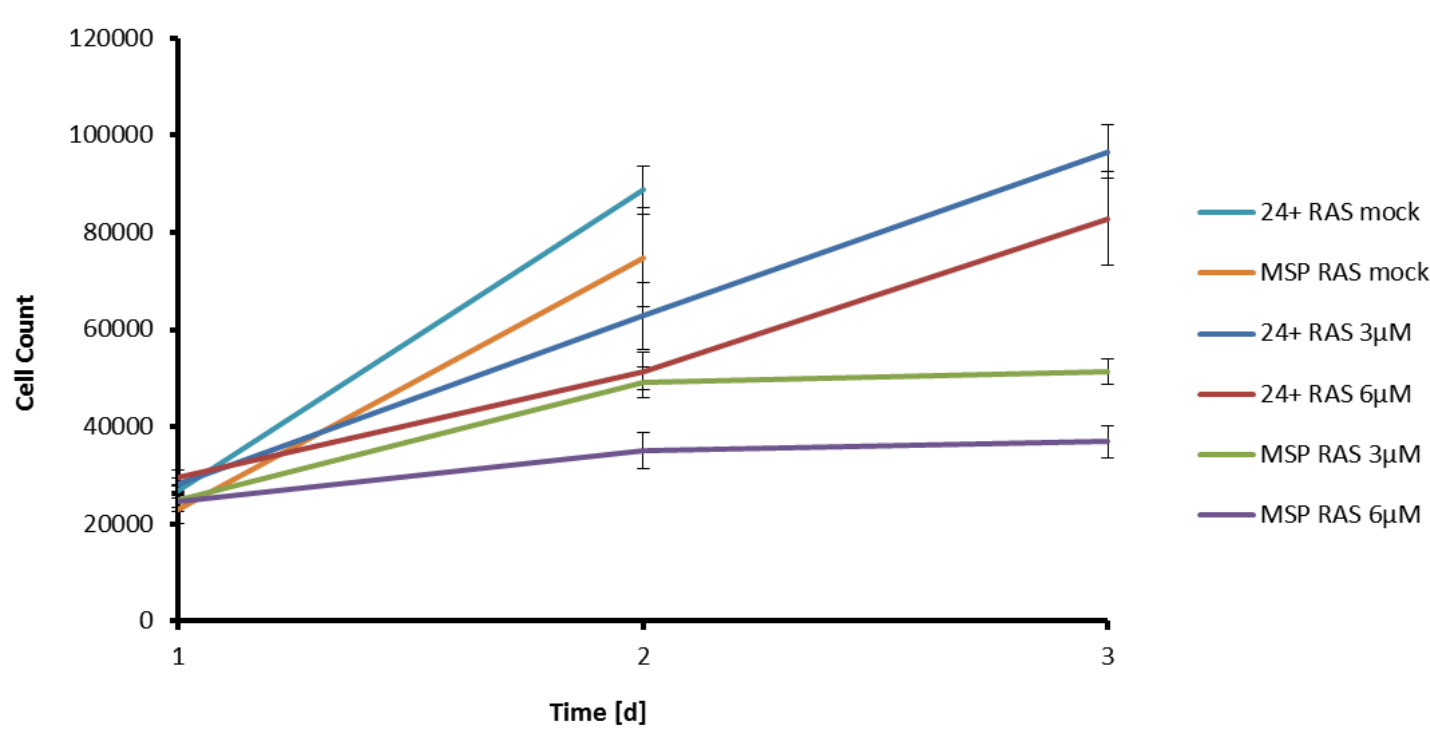

Figure 8.1: MSP RAS cells are sensitive to Salinomycin treatment.

24h after seeding HMLE RAS cells were treated with $3 \mu \mathrm{M}$ or $6 \mu \mathrm{M}$ Salinomycin. Cell number was measured once a day for $3 \mathrm{~d}$ with a Celigo ${ }^{\circledR}$ cytometer after adding fresh Salinomycin. Non-treated cells were used as control. Data show mean \pm SD. $n=1$. 